\title{
A numerical and experimental study of the effects of dynamic roughness on laminar leading edge separation
}

\author{
Peter D. Gall \\ West Virginia University
}

Follow this and additional works at: https://researchrepository.wvu.edu/etd

\section{Recommended Citation}

Gall, Peter D., "A numerical and experimental study of the effects of dynamic roughness on laminar leading edge separation" (2010). Graduate Theses, Dissertations, and Problem Reports. 2984.

https://researchrepository.wvu.edu/etd/2984

This Dissertation is protected by copyright and/or related rights. It has been brought to you by the The Research Repository @ WVU with permission from the rights-holder(s). You are free to use this Dissertation in any way that is permitted by the copyright and related rights legislation that applies to your use. For other uses you must obtain permission from the rights-holder(s) directly, unless additional rights are indicated by a Creative Commons license in the record and/ or on the work itself. This Dissertation has been accepted for inclusion in WVU Graduate Theses, Dissertations, and Problem Reports collection by an authorized administrator of The Research Repository @ WVU.

For more information, please contact researchrepository@mail.wvu.edu. 


\title{
A NUMERICAL AND EXPERIMENTAL STUDY OF THE EFFECTS OF DYNAMIC ROUGHNESS ON LAMINAR LEADING EDGE SEPARATION
}

\author{
Peter D. Gall
}
Dissertation submitted to the College of Engineering and Mineral Resources at West Virginia University in partial fulfillment of the requirements for the degree of

\author{
Doctor of Philosophy \\ in \\ Aerospace Engineering
}

Approved by

Dr. Wade Huebsch, Committee Chairperson

Dr. John Loth

Dr. Jagannath Nanduri

Dr. Mary Anne Clarke

Dr. Gary Morris

Mr. William Sewall

Mechanical and Aerospace Engineering

Morgantown, West Virginia

2010

Keywords: dynamic roughness, separation bubble, computational fluid dynamics, flow control 


\begin{abstract}
A Numerical and Experimental Study of the Effects of Dynamic roughness on Leading Edge Separation

Peter D. Gall
\end{abstract}

The aircraft industry, as a whole, has been deeply concerned with improving the aerodynamic efficiency of current and future flight vehicles, particularly in the commercial and military markets. However, of particular interest to the field of aerodynamics is the elusive concept of a workable flow control mechanism. Effective flow control is a concept which if properly applied can increase aerodynamic efficiency. Various concepts and ideas to obtain successful flow control have been studied in an attempt to reap these rewards. Some examples include boundary layer blowing (steady and periodic), suction, and compliant walls for laminar flow control. The overall goal of flow control is to increase performance by increasing lift, reducing drag, and delaying or eliminating leading edge separation. The specific objectives of flow control are to 1) delay or eliminate flow separation 2) delay boundary layer transition and 3) and reduce skin friction drag. The purpose of this research is to investigate dynamic roughness as a novel method of flow control technology for external boundary layer flows. As opposed to standard surface roughness, dynamic roughness incorporates small time dependent perturbations to the surface of the airfoil. These surface perturbations are actual humps and/or ridges on the surface of the airfoil that are on the scale of the laminar boundary, and oscillate with an unsteady motion. Research has shown that this can provide a means to modify the instantaneous and mean velocity profile near the wall and favorably control the existing state of the boundary layer. Several flow control parameters were studied including dynamic roughness frequency, amplitude, and geometry. The results of this study have shown, both numerically and experimentally, that dynamic roughness can provide an effective means for eliminating both a short and long laminar separation bubble and possibly prove a viable alternative in effective flow control, hence reaping some of the rewards of an effective flow control system. 


\section{Acknowledgments}

I would like to express my sincere gratitude to my research advisor, Dr. Wade Huebsch, for his teaching, guidance, and support during this research. He has not only been my research adviser, but my mentor and friend.

I would also like to thank the members of my committee, Dr. John Loth, Dr. Jaggu Nanduri, Dr. Gary Morris and Dr. Mary Ann Clarke for their invaluable suggestions and insights. I would also like to especially thank Mr. Bill Sewall, my former colleague at NASA Langly, for his advise and for the many late night discussions that we had.

I would like to thank Dr. Jacky Prucz for encouraging me to pursue my life long dream, to obtain a $\mathrm{PhD}$ in Aerospace Engineering and teach my favorite subject, aircraft design.

I would also like to thank Shanti Hamburg for his assistance in the experimental part of this study and Pilar Tanning, my fiancé, for allowing me to work undisturbed on this research effort. 


\section{Contents}

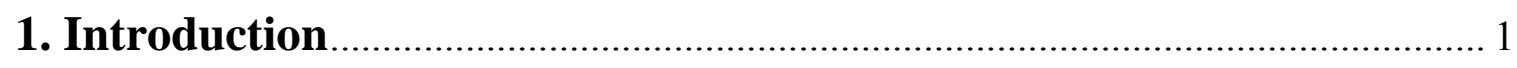

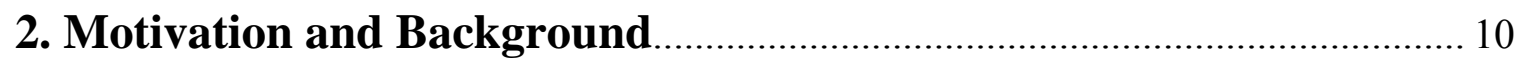

2.1 Statement of Problem and Motivation of Study .................................................. 10

2.2 Past CFD Analysis of Leading Edge Separation Bubbles .................................... 16

2.3 Review of Past Separation Control .............................................................. 20

3. Flow Physics of Isolated and Distributed Roughness ............................. 27

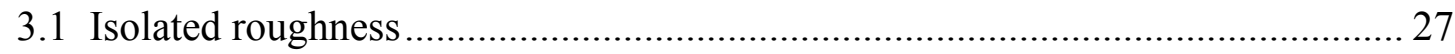

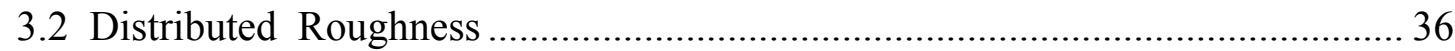

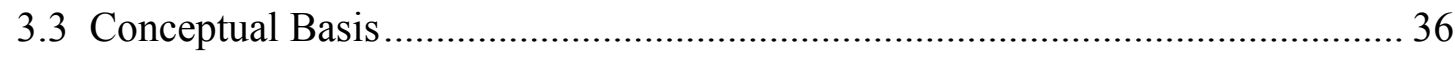

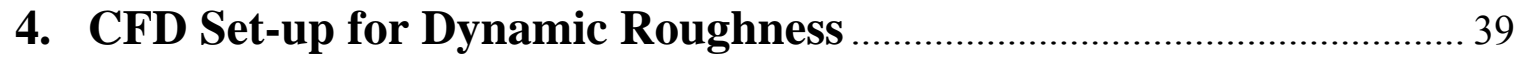

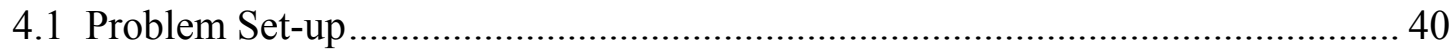

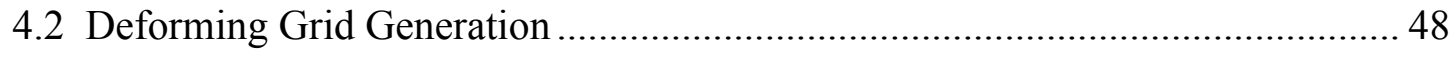

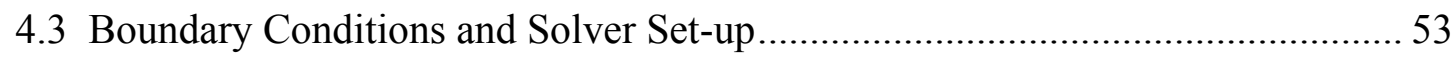

5. Computational Fluid Dynamics Analysis of Leading Edge Flow Separation and Dynamic Roughness …………………......................5 57

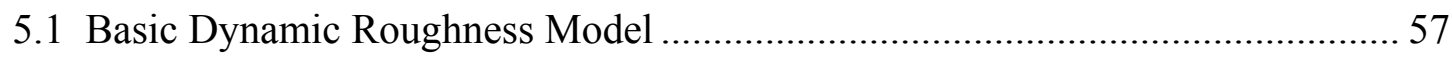

5.2 Comparison of Two-dimensional and Three-dimensional Analysis ..................... 86

5.3 Amplitude and Frequency Effects on Dynamic Roughness Flow Control.......... 87

6. Experimental Analysis of Dynamic Roughness ………………………..... 93

6.1 Wind Tunnel Description and Set-up................................................................. 93

6.2 Dynamic Roughness Wind Tunnel Model........................................................... 97 


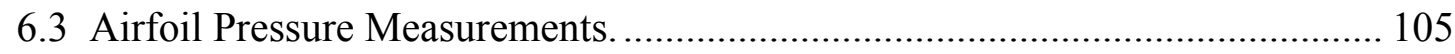

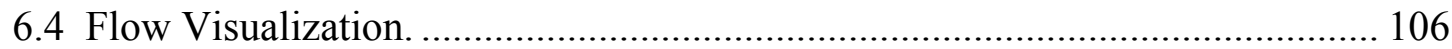

7. Comparison of Experimental and Numerical Results ......................... 110

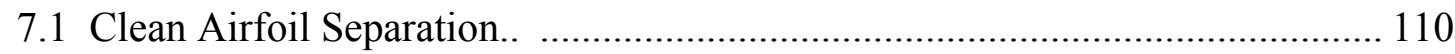

7.2 Short Leading Edge Separation Bubble. ................................................... 115

7.3 Long Leading Edge Separation Bubble .................................................... 121

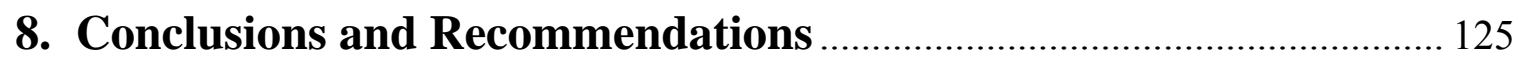

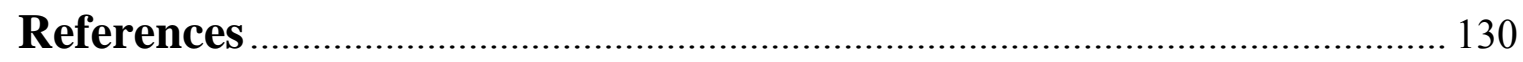

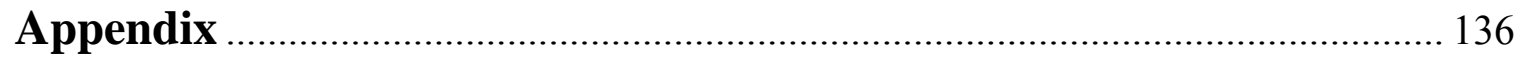




\section{List of Figures}

Figure 1.1: Typical geometry of two-dimensional dynamic roughness hump [Huebsch, 2006] 5

Figure 1.2: Futuristic wing featuring dynamic roughness ........................................ 8

Figure 2.1: Fundamental stall patterns of an airfoil [McCullough and Gault, 1955] ...... 13

Figure 2.2: Depiction of a typical separation bubble [Alam and Sandham, 2000]. ......... 14

Figure 2.3: Typical pressure distributions of a long and short separation bubble [Rinoie and Takemaura, 2004 and Roberts, 1980]

Figure 2.4: Oscillating lift and drag on a NACA 0012 airfoil at 10 degrees angle of attack and Reynolds number of 100,000 [Nakae et al., 2006].

Figure 2.5: Leading edge region of numerical analysis of a two-dimensional separation bubble at 9.5 degrees angle of attack and a Reynolds number of 100,000 (current research). 20

Figure 3.1: Increase in zero lift drag due to surface roughness [Braslow, 1966] 30

Figure 3.2: Illustration of three-dimensional vortex structure [Acalar and Smith, 1987]

Figure 3.3: Strouhal number as a function of roughness Reynolds number for semihemispheric roughness (digitized from Acarlar and Smith, 1987) .............................. 34

Figure 3.4: Effect of critical Reynolds number on flow [Matheis et al., 2004]. .............. 35

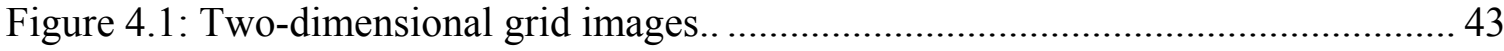

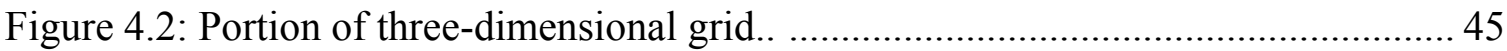

Figure 4.3: Planer cut of three-dimensional dynamic roughness mesh. ........................ 46

Figure 4.4: Surface depiction of surface humps and ridges on airfoil leading edge........ 47

Figure 4.5: Close-up of a dynamic roughness hump... ........................................... 48

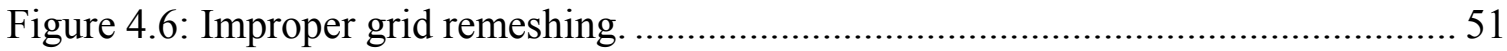

Figure 4.7: Acceptable grid remeshing in roughness region. ................................... 52 
Figure 5.1: Clean airfoil and dynamic roughness airfoil leading edge pressure distributions.

Figure 5.2: Clean airfoil and dynamic roughness airfoil pressure distributions (time and spaced averaged)..

Figure 5.3: Pressure distributions for clean and dynamic roughness wings throughout hump cycle in the leading edge region. 64

Figure 5.4: Pressure coefficient over first two humps throughout hump cycle. 66

Figure 5.5: Time history of hump motion. 66

Figure 5.6: Displacement history of hump motion..

Figure 5.7: Experimental and numerical pressure coefficient data for 9.5 degrees angle of attack and Reynolds number 100,000 68

Figure 5.8: Boundary layer velocity profile at $0.6 \%$ chord location. 70

Figure 5.9: Boundary layer velocity profile at $1.8 \%$ chord location. 71

Figure 5.10: Boundary layer velocity profile at $3.4 \%$ chord location. 72

Figure 5:11: Surface pressure contour and pathline plots of clean airfoil and airfoil with dynamic roughness ridges ( 12 degrees angle of attack and $\operatorname{Re}$ of 100,000) 74

Figure 5.12: Pressure contour and pathlines of a) clean, b) static, and c) dynamic roughness airfoils at time $t_{1}$. 78

Figure 5.13: Pressure contour and pathlines of a) clean, b) static, and c) dynamic roughness airfoils at time $\mathrm{t}_{2}$

Figure 5.14: Pressure contour and pathlines of clean, static, and dynamic roughness airfoils at time $t_{3}$.

Figure 5.15: Three-dimensional pathline plots of clean, static and dynamic roughness cases. 82

Figure 5.16: Wall shear stress values for clean laminar, clean turbulent, and dynamic roughness laminar wings.

Figure 5.17: Vorticity and pathlines in region of first and last humps during dynamic roughness cycle.. 
Figure 5.18: Two-dimensional dynamic roughness case at 12 degrees angle of attack and 100,000 Reynolds number.

Figure 5.19: Flow control analysis as a function of roughness amplitude and frequency at 12 degrees angle of attack and Re of 100,000.

Figure 5.20: Flow control analysis as a function of roughness amplitude and Strouhal number at 12 degrees angle of attack and Re of 100,000.. 92

Figure 6.1: Side view of WVU smoke tunnel. 94

Figure 6.2: Wind tunnel test section velocities..... 95

Figure 6.3: Wind tunnel turbulence measurements. 97

Figure 6.4: Dynamic roughness model substructure. 99

Figure 6.5: Completed dynamic roughness apparatus. 100

Figure 6.6: Dynamic roughness apparatus with pressure applied. 101

Figure 6.7: Final assembly of dynamic roughness model. 104

Figure 6.8: Pressure taps on model. 105

Figure 6.9: Modified commercial smoke generation system. 106

Figure 6.10: Smoke discharge rake. 107

Figure 6.11: Pictorial of air-smoke generation system built by the author. 108

Figure 6.12: Oil-air smoke generation system.

Figure 7.1: Lift curve slope for NACA 0012 at Reynolds numbers of 100,000 and 3,000,000 [Abbot and Doenhoff, 1956 and Jacobs, 1938].

Figure 7.2: Flow visualization of baseline airfoil showing attached flow, separation bubble, and leading edge stall at Re of 100,000.

Figure 7.3: Separation bubble at 9.5 degrees angle of attack without dynamic roughness actuation.

Figure 7.4: Separation bubble at 9.5 degrees angle of attack and $30 \mathrm{~Hz}$ dynamic roughness frequency actuation. 
Figure 7.5: Separation bubble eliminated and attached flow achieved at 9.5 degrees angle of attack and $60 \mathrm{~Hz}$ dynamic roughness frequency actuation.

Figure 7.6: Pressure distributions for clean and dynamic roughness airfoil at 9.5 degrees angle of attack and a Reynolds number of 100,000 (experiment) and 130,000 [Rinoi and Takemura, 2000].

Figure 7.7: Separation bubble at 12.0 degrees angle of attack a without dynamic roughness actuation.

Figure 7.8: Separation bubble at 12.0 degrees angle of attack with dynamic roughness not actuated (red jagged lines bear no significance and are divisions in cell domain).

Figure 7.9: Pressure distributions for clean and dynamic roughness airfoil at 12.0 degrees angle of attack and a Reynolds number of 100,000 (experiment) and 130,000 [Rinoi and Takemura, 2000]. 


\section{List of Tables}

Table 4.1: Grid smoothing and remeshing parameters................................................53

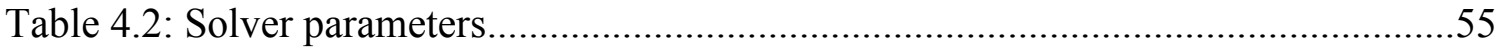

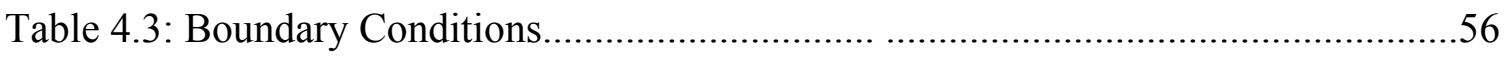

Table 5.1: Clean Airfoil Dynamic Roughness Airfoil Geometric Parameters..................73

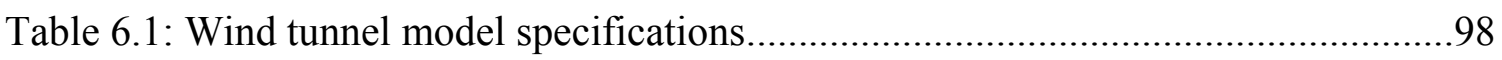

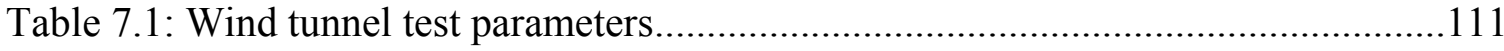




\section{Chapter 1}

\section{Introduction}

The aerospace community is continually searching for methods to improve the aerodynamic efficiency of current and future flight vehicles, particularly in the commercial and military markets. Upon reviewing recent emerging technologies, it is apparent that there have been many advancements in several areas of design such as aerodynamics, structures, propulsion, and controls. However, of particular interest to the field of aerodynamics is the elusive concept of a workable flow control mechanism. This is particularly important for low Reynolds number airfoil ranges where laminar separation bubbles become an ever present phenomenon. Many airfoil applications fall into this range such as mid and high altitude UAV's, sailplanes, jet engine fan blades, inboard helicopter rotor blades, wind turbine rotors, and propellers at high altitudes. Also, there has been recent interest in micro-air vehicles (MAV's) which also fall into this range. To apply flow control means altering the flow field over an airfoil or body in order to improve its efficiency.

Many conceptual solutions to the flow control problem have been proposed, some holding a much greater potential for successful implementation than others. There are, however, several reasons why most of these concepts have not been implemented into mainstream manufacturing. Some systems actually have a higher power usage requirement than power savings, resulting in a net energy loss. An example of this would be flow control system using boundary layer suction. Although use of the 
system may result in a reduction of the aerodynamic drag, the energy required to operate the suction pump would be a direct tax on the amount of fuel burned. The fuel required to power the system may be greater than the amount of fuel that is saved due to the reduction in drag. Hence, the overall efficiency of the system may actually be decreased

Another issue with some flow control systems is they have a very narrow operating envelope for control authority; when flow control is attempted in off-design conditions, the airfoil flow control system fails. Also, in some cases the high cost or complexity of the airfoil flow control system is simply not feasible. In other cases the system appears operational in the lab environment, but experiences difficulties when applied in the field. Therefore, designing a mechanism which can overcome these fundamental flaws could prove a significant advancement in improving the aerodynamic efficiency of flight vehicles.

The global goals of flow control are to increase performance by increasing lift, reducing drag, and improving the stall characteristics of a given airfoil or wing. The specific objectives of flow control are usually achieved through one or more of the following: 1) delay or eliminate flow separation 2) delay boundary layer transition or 3) reduce skin friction drag. From an aerodynamic standpoint, proper flow control mechanisms have the potential to decrease skin friction and form drag, increase lift, improve flight controllability and maneuverability, and provide significant savings in overall fuel consumption. For example, maintaining laminar flow over the entire wing 
surface can reduce total aircraft drag by as much as $15 \%$ [Schrauf, 2000]. In the commercial aircraft industry, an overall drag reduction of just $1 \%$ can translate to millions of dollars saved in annual fuel costs. Various concepts and ideas to obtain successful flow control have been studied in an attempt to reap these rewards. Some examples include boundary layer blowing (steady and periodic), suction, synthetic jets and compliant walls for laminar flow control. For turbulent flows, the addition of riblets and compliant walls to the airfoil, bubble injection, and polymer additions to the surface flow have been studied. Also, recent studies have been done using plasma injections. These techniques have met with varying levels of success.

The purpose of this research is to investigate dynamic surface roughness as a novel method of flow control technology for external boundary layer flows. As opposed to normal surface roughness (a result of manufacturing imperfections or insect debris for example), dynamic roughness incorporates small time dependent perturbations to the surface of the airfoil. These surface perturbations are actual humps and/or ridges on the surface of the airfoil that are on the scale of the local boundary layer, but with an unsteady motion. When dynamic roughness amplitude is smaller than or comparable to the height of the existing boundary layer it has been shown to provide a means to modify the instantaneous and mean velocity profile near the wall and control the local state of the boundary layer. This can lead to the suppression of the leading edge separation bubble. The author believes that some possible explanations for this flow control mechanism are: 1) the alteration of flow instabilities, 2) the creation of hairpin type vortices in the viscous sub layers of the boundary layer which enhances mixing 
and entrainment, 3) the creation of artificial Reynolds stresses, and 4) favorable alterations of the pressure gradient (or a combination of the above).

When dynamic roughness is on the same order as the height of the boundary layer, it tends to completely alter the state of the boundary layer. For example, when a laminar boundary layer is forming on the leading edge of an airfoil under certain conditions of Reynolds number and angle of attack, a separation bubble will normally form. As a specific example, for a NACA 0012 at 12 degrees angle of attack and a Reynolds number of 100,000 separation will occur at about the $1.8 \%$ chordwise location. If dynamic roughness is sized on the scale of the approaching boundary layer and introduced just upstream of the separation point, the state of the approaching boundary layer will be altered prior to it reaching the natural separation point [Huebsch, 2006]. This altered state is completely different from the laminar boundary layer which originally tended towards separation. This was evident in the results of the experimental part of this study. This new effective state has different separation, stability, and transition properties, and is expected to produce surface forces that are significant enough to alter the lift and drag characteristics of the airfoil. This study has demonstrated that dynamic roughness, if correctly applied, can suppress separation and increase the efficiency of a given airfoil or wing.

The current research anticipates that the dynamic roughness can be tuned to create a number of flow control scenarios. Low amplitude oscillations may alter flow instabilities, transition, and turbulent sublayers. Larger scale oscillations may locally 
control flow separation, which could ultimately provide a means for direct surface pressure control of lift, which in turn, can directly affect in-flight maneuverability

Dynamic roughness has the potential for applications in other areas of aerodynamics as well as other industries where unique flow applications are encountered. An example of these would be internal channel flows or dust on space suits. Figure 1.1 depicts the geometry of a two-dimensional dynamic roughness hump.

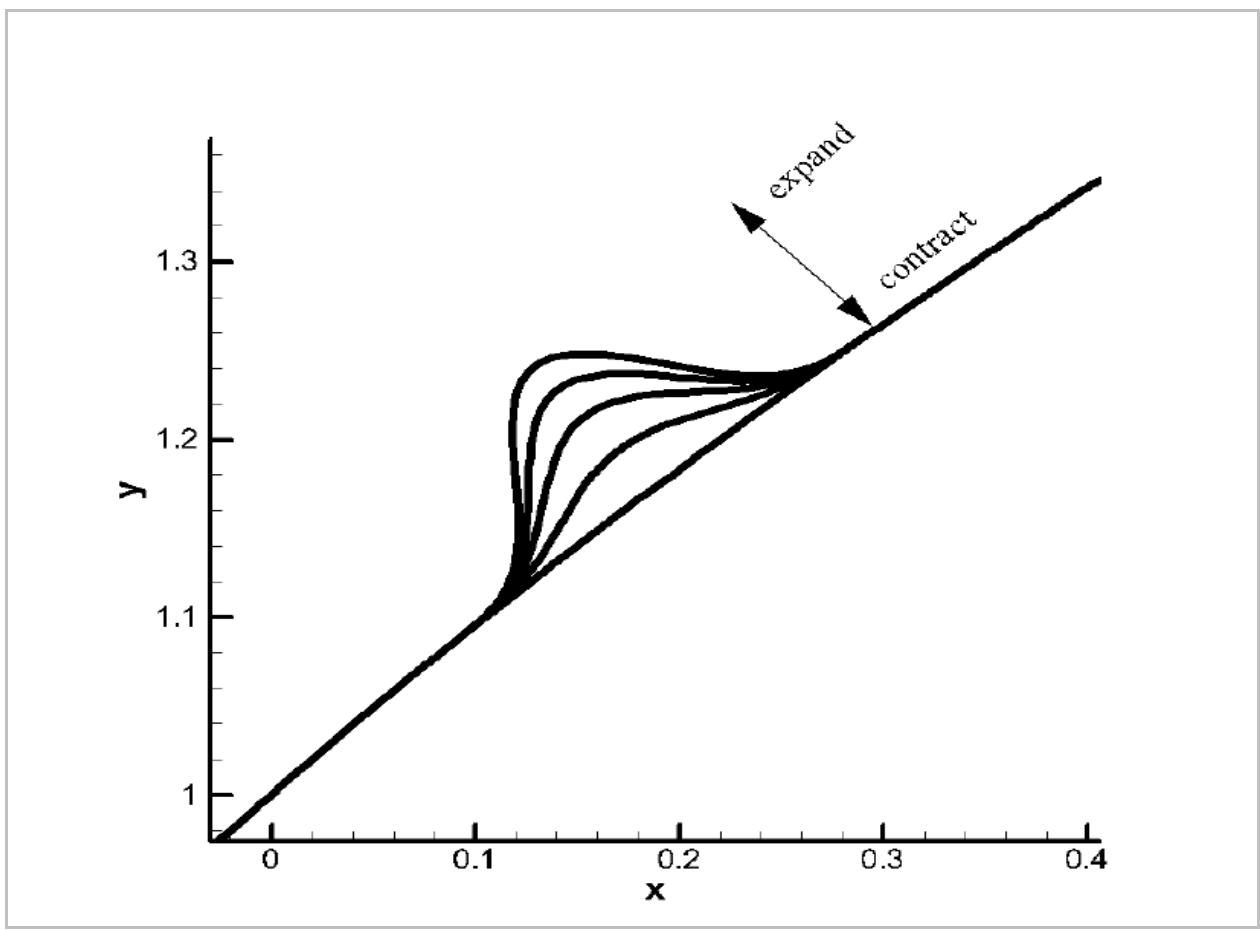

Figure 1.1: Typical geometry of two-dimensional dynamic roughness hump [Huebsch, 2006]

Preliminary results using a two-dimensional Navier-Stokes algorithm have shown that dynamic roughness can control laminar flow separation for external flows [Huebsch, 2006]. It was also found that dynamic roughness can produce a significant change in the flow physics near the leading edge of an airfoil when compared to static 
roughness with comparable roughness height. Various simulations were also studied in order to examine the effects of dynamic roughness on the dynamic stall maneuver. The dynamic stall was selected as an extreme type of unsteady flow behavior due to the inherent vortex formation. Dynamic roughness suppressed the formation of the dynamic stall vortex, which is significant given the fact that the vortex formation is rather robust. The effect of dynamic roughness was to shed smaller coherent vortex structures, which had a smaller length scale than the boundary layer height. Twodimensional simulations have shown that these vortices tend to be benign and may even possibly be favorable due to the mixing effect in the sublayers of the viscous boundary layer close to the wall. Key findings of the two-dimensional work were; 1) the roughness needs to be located just upstream or in the vicinity of the separation point; 2) if the frequency is too low the flow sees the dynamic roughness as merely another form of static roughness and; 3) the maximum amplitude of the dynamic roughness needs to be less than that of the local boundary layer.

The current study initially focused on simulating a two-dimensional roughness case similar to the previous work, but using the commercial code Fluent ${ }^{\circledR}$ as the next step in validation of this mechanism. This code utilizes a finite volume Navier-Stokes implicit type solver. Several user defined function algorithms (UDF's) were developed that simulated the motion of the dynamic roughness (see Sec. 4.2). Results of the two-dimensional study were comparable to the results found in the work of Huebsch [2006]. The second numerical phase was to develop a fully functional threedimensional model, also utilizing Fluent ${ }^{\circledR}$. Again, several UDF algorithms were 
developed representing various geometries such as spanwise ridges and spanwise humps consisting of various geometric heights, shapes, and frequencies.

Previous direct numerical simulation (DNS) work has shown that the vorticity created by static humps is highly three-dimensional in nature, creating "hairpin" type coherent vortex structures which convect downstream within the boundary layer [Matheis et al., 2004]. Therefore, it would seem logical that based on the static roughness study, dynamic roughness would also be highly three-dimensional in nature. Interestingly enough, in this research study, the three-dimensional analysis showed results similar to the two-dimensional analysis; that dynamic roughness can greatly alter the boundary layer in a favorable way which can result in delaying and/or eliminating the separated flow region. Therefore, the three-dimensional analysis appeared to be a prudent research application.

Dynamic roughness has the potential for many applications on an actual flight vehicle. Figure 1.2 illustrates several areas where dynamic roughness may be used to improve certain aerodynamic and operational characteristics. The figure illustrates what a futuristic wing may look like incorporating dynamic roughness. 


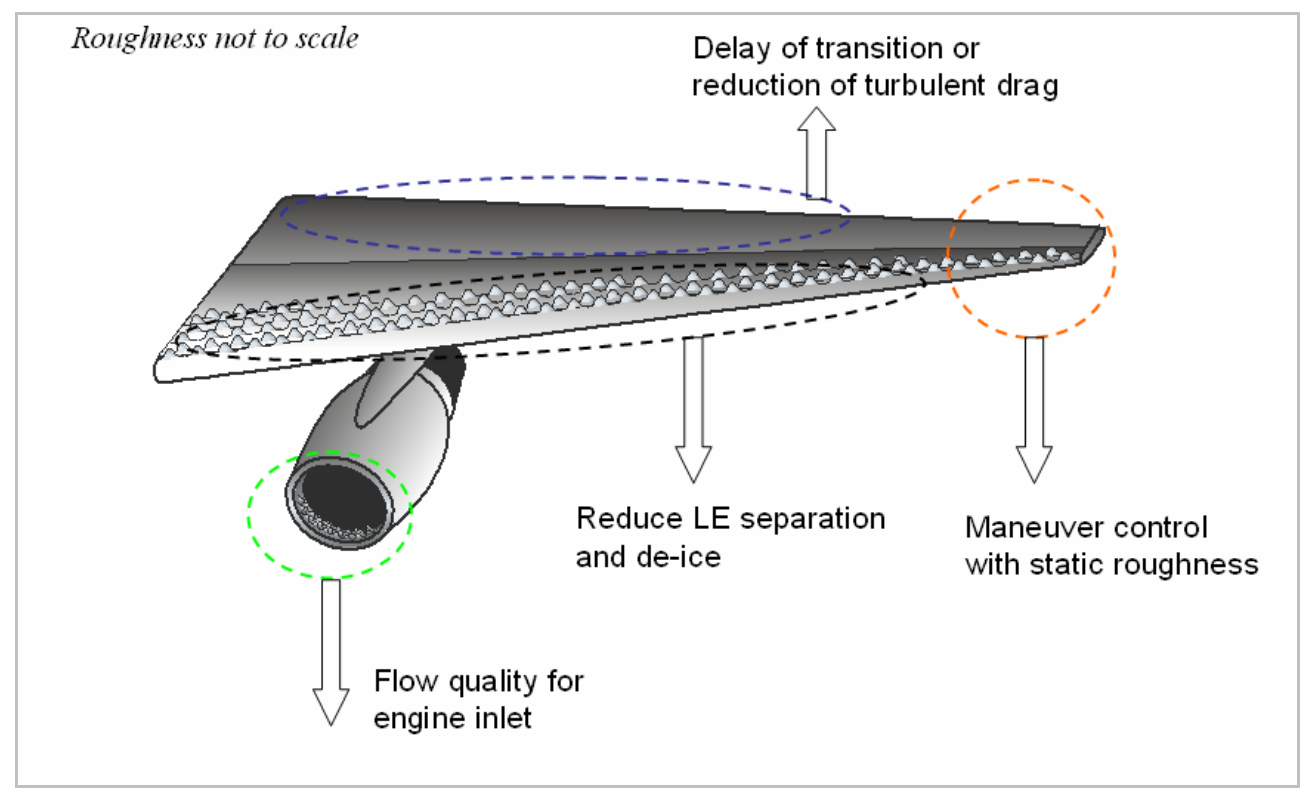

Figure 1.2: Futuristic wing featuring dynamic roughness

As a final point in introducing the concept of dynamic roughness, a comparison was made with past flow control mechanisms. Dynamic roughness appears to offer several potential advantages over other methods of flow control.

Dynamic roughness offers a means for a surface mounted flow control device. It avoids the potential problem of holes in a surface that may become clogged.

Dynamic roughness will not alter the structural integrity of the existing surface.

The overall implementation of dynamic roughness benefits from a reduction in surface modifications, complex plumbing, etc. 
Dynamic roughness can always return to a neutral position and provide a "clean" aerodynamic surface.

$>$ The dynamic roughness can be used to produce a constant roughness, or static type roughness, if needed. This technique gives it an additional degree of freedom, possibly for different modes of flow control.

$>$ The anticipated power requirements will be at or lower than the levels for synthetic jets or plasma actuators.

$>$ Dynamic roughness will be able to provide a range of amplitude heights, both static and dynamic, as well as a range of frequencies.

$>$ Dynamic roughness may provide additional benefits such as maneuvering capabilities and in-flight deicing.

Dynamic roughness is "tunable" in that it can provide flow control over a range of flight regimes. 


\section{Chapter 2}

\section{Motivation and Background}

\subsection{Statement of Problem and Motivation of Study}

The basic problem addressed in this research study was the phenomenon of leading edge separation and its inherent adverse affects on performance. Given a typical airfoil or wing section operating at an angle of attack and producing lift there exists an adverse pressure gradient downstream of the stagnation point on the upper surface. If the adverse pressure gradient is significant, the oncoming boundary layer flow is laminar and the Reynolds number is low enough the boundary layer tends toward separation, a phenomenon that is commonly referred to as the "leading edge separation bubble." The separation bubble creates a separated shear layer which is inherently unstable. As a result of this instability and the resulting velocity distributions, the laminar boundary layer begins transitioning to a turbulent state. Once the transition takes place, the turbulent shear stresses begin to energize this shear layer by entraining fluid from the outer stream. This phenomenon redistributes the energy in the higher momentum outer flow bringing it closer to the surface. Reattachment is typically believed to occur when the surface pressure is nearly equal to that which would exist if the flow had been turbulent over the airfoil surface with no separation bubble present. For this reason, the inviscid solution of the pressure distribution is sometimes used to predict the reattachment location since it approximates the turbulent boundary layer case. 
The separation bubble is referred to as the region between the separation point and reattachment point. The same factors which affect the boundary layer separation also affect the separation bubble itself as well as the transition process. These factors are most notably the Reynolds number, angle of attack, boundary layer thickness, pressure distribution, and free stream turbulence levels. The flow physics of how the reattached turbulent boundary layer grows or develops over the remaining portion of the airfoil is highly dependent on the transition process in the separated shear layer. Much is known about the transition of boundary layers when the shear layer is attached, but much less is known about the transition process in separated shear layers. Studies have been done exploring rearward facing steps and free jets, but the leading edge separation bubble is unique in that its creation is primarily due to an adverse pressure gradient [Mueller and Batill, 1982]. The flow physics of this type of bubble and its corresponding shear layer is primarily dependent on the interaction between the airfoil boundary layer and the external flow field. There are some similarities in these different types of cases but there are many more differences when considering the development of the shear layer.

Early on, researchers at NACA (the predecessor to NASA) began exploring the various mechanisms which may exist leading up to a stall. They soon discovered that whether or not the boundary layer transitions to turbulent flow prior to separation has a pronounced effect on the stall characteristics. They classified the stall into three fundamental types: 1) trailing edge stall 2) leading edge stall and 3) thin airfoil stall. 
When the boundary layer separates prior to transition, a separation bubble is formed. When the separation bubble first forms, it separates near the leading edge and typically reattaches a short distance downstream. This is referred to as the "short" bubble. The existence of the short bubble does not significantly alter the global lift and drag characteristics. It is a characteristic of most airfoils of moderate thickness $(9 \%-15 \%)$ operating at low Reynolds numbers (less than 500,000). This separation is coincident with the adverse pressure gradient downstream of the location of maximum suction. The flow separates in a laminar state and transitions to a turbulent state. The bubble typically reattaches when the local pressure approximates the pressure that would exist if the flow were attached (inviscid case). Also, as the angle of attack is increased the bubble tends to move forward and the region of reverse flow increases.

At certain values of Reynolds number and angle of attack the turbulent mixing and entrainment processes within the short bubble can no longer increase the negative pressure coefficient to a value high enough for reattachment to occur. At this point, the short bubble is said to "burst", forming a long bubble. The long bubble may reattach much further downstream or not reattach at all. The bursting phenomenon causes an abrupt loss of lift, increase in drag, and a pronounced change in the pitching moment [McCullough and Gault, 1955]. This type of stall is referred to as the leading edge stall. Figure 2.1(b) depicts a typical lift curve and pathline illustration of a leading edge stall.

In other cases, when an airfoil has a low thickness ratio or a sharp leading edge, another type of stall occurs. This is referred to as a thin airfoil stall. As the angle of attack is 
increased, a separation occurs near the leading edge. This separation resembles a standing vortex. In this stall case the flow typically reattaches a short distance downstream. As angle of attack is increased or Reynolds number decreased, the reattachment point moves downstream, and the lift gradually decreases . A typical lift curve and pathline illustration of this type of stall is also shown in figure 2.1(c).

The last type of stall occurs when the transition of the boundary layer occurs upstream of where the laminar separation point would be. The flow, in this case, becomes turbulent and begins separating near the trailing edge. It is typical of most airfoils operating at higher Reynolds numbers (greater than 3,000,000). This is referred to as the trailing edge stall. Figure 2.1(a) shows a typical lift curve and pathline illustration for this type of stall.

a) Trailing edge stall
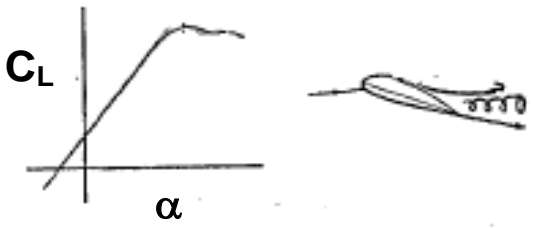

b) Leading edge stall
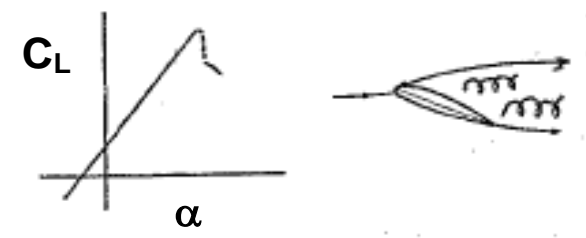

c) Thin airfoil stall
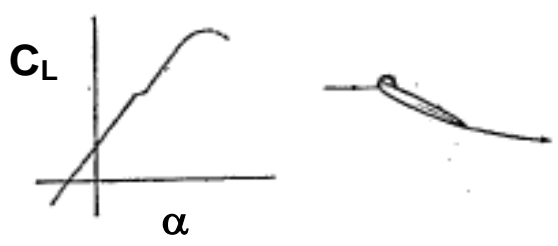

Figure 2.1: Fundamental stall patterns of an airfoil [McCullough and Gault, 1955] 
Figure 2.2 depicts an exaggerated view of what a short separation bubble may look like.

Beneath the shear layer is a line of demarcation separating the laminar and turbulent shear layers and the "dead air" region located within the bubble. The vortex formation within the bubble is also shown. This vortex s highly unsteady and serves as a mixing and entrainment mechanism.

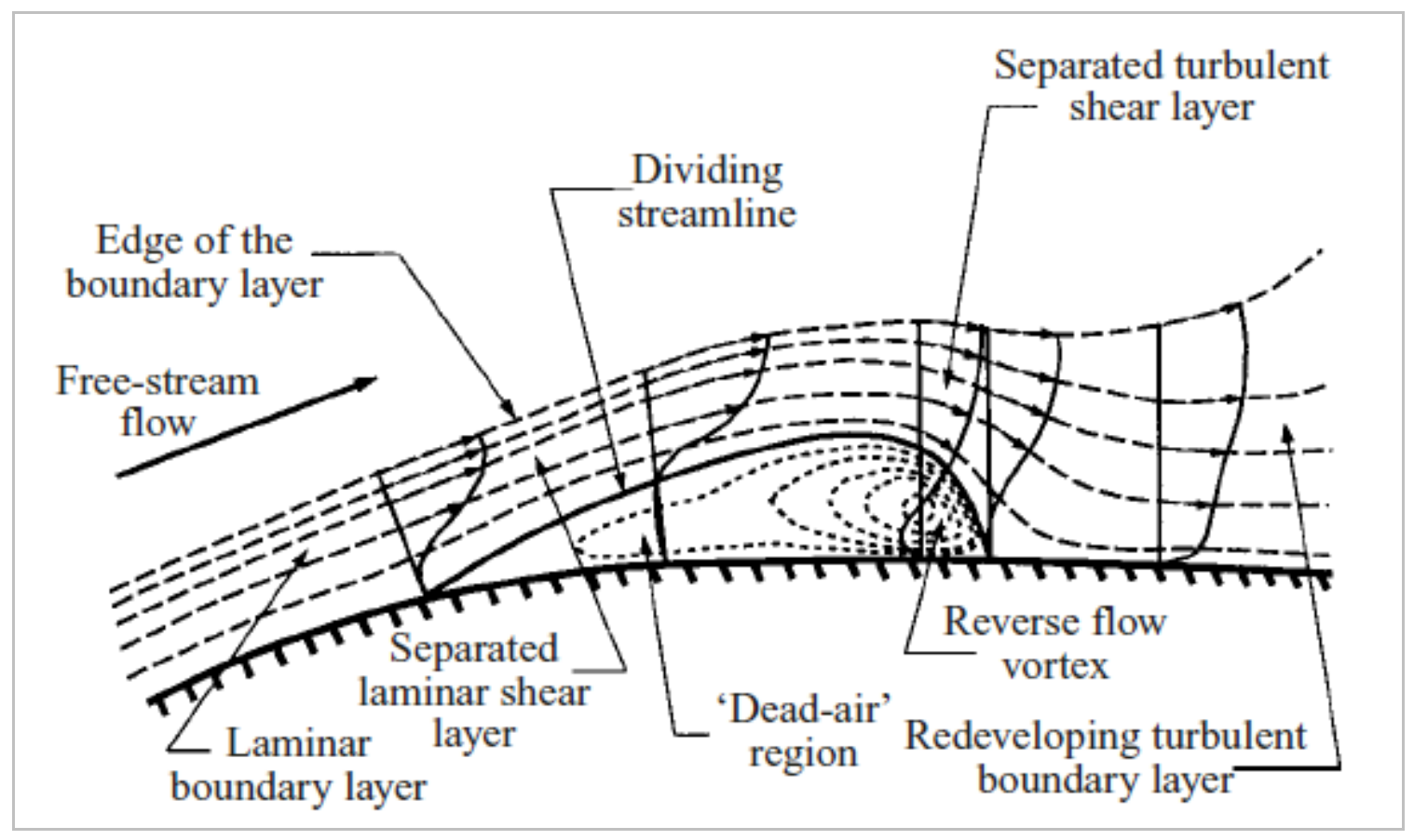

\section{Figure 2.2: Depiction of a typical leading edge separation bubble [Alam and} Sandham, 2000]

Figure 2.3 depicts typical pressure distributions observed when a long or short separation bubble exists. Although the short bubble does not significantly alter the global lift and drag, both bubbles can alter the pressure distribution. The short bubble, depending on its characteristics, can cause a slight increase in the suction pressure gradient followed by a plateau and a normal pressure recovery. The long bubble, on the other hand, tends to 
collapse the suction pressure over a large region. This long bubble clearly has a pronounced effect on the global lift and drag.

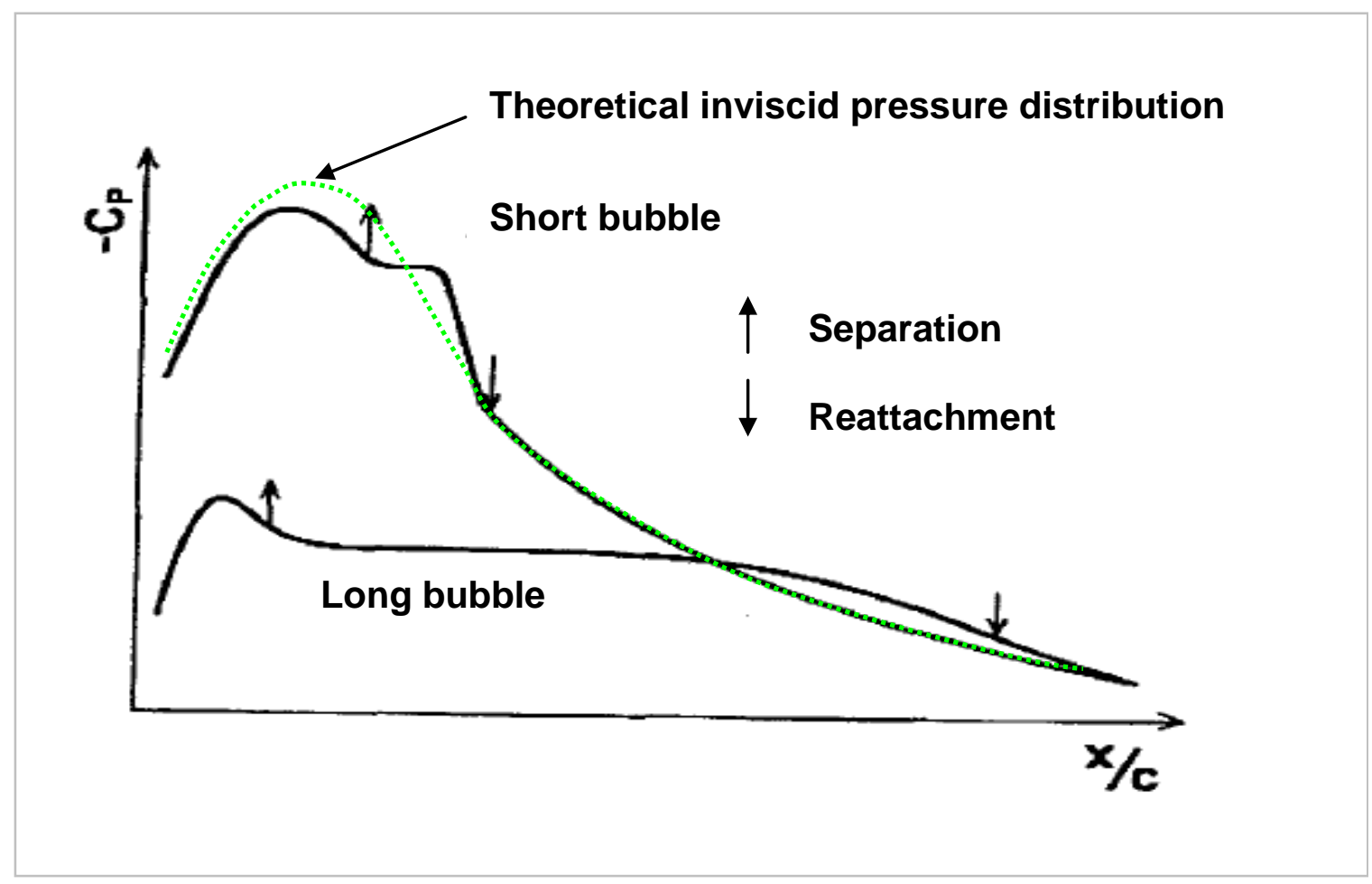

Figure 2.3: Typical pressure distributions of a long and short bubble separation [Rinoie and Takemaura, 2004 and Roberts, 1980]

If the Reynolds number is increased sufficiently, the transition location can move forward to where it is ahead of the point where laminar separation would occur. This, in essence, creates a turbulent boundary layer which now may prevent the bubble from forming at all. This critical Reynolds number is a function of pressure distribution along the surface, surface roughness, and free stream turbulence. Transition can also occur artificially by adding trips near the leading edge of the airfoil. This is often done to eliminate the separation bubble (turbine blades and model aircraft). Either of these means 
will produce a pressure distribution similar to the flow solution for a turbulent boundary layer.

The basic problem is that of reducing or eliminating the laminar separation bubble when

it exits. In doing so, the leading edge stall may be eliminated and the thin airfoil stall may be delayed or eliminated resulting in higher lift and lower drag. Dynamic roughness has been shown in this study to offer a method of accomplishing this during the portions of the flight envelope where an existing separation bubble would penalize lift and drag performance. This has the potential to greatly improve the efficiency of an airfoil.

\subsection{Past CFD Analysis of Leading Edge Separation Bubbles}

Early computational fluid dynamics studies of the laminar separation bubble using a Navier-Stokes type solver showed that the flow in the laminar region of the bubble undergoes a periodic shedding of vortical structures [Alam and Sandham, 2000]. Alam and Sandham [2000] performed direct numerical simulations (DNS) on a laminar separation bubble. The results suggested that the bubble bursting may be marked by the difference between an absolutely unstable long bubble and a convectively unstable short bubble, depending on the amount of reversed flow. They analyzed only short bubbles, and therefore no distinctive conclusions were drawn based on these suggestions. They did, however, find that unsteady coherent structures within the bubble were found to persist and seemed to characterize the ensuing flow development, particularity at low Reynolds numbers. These results provided a bubble model different from the classical approach, which depicts the bubble as a region of stagnant flow 
between the separation and reattachment point. This also provided an explanation as to why the classical approach fails in predicting a bursting occurrence [Horton, 1969].

The pressure distributions obtained by time averaging the unsteady results were found to match selected experimental data [Mueller and Batill, 1982]. Also, the time averaged streamlines were found to reproduce the classical bubble pattern shown in figure 2.2 This certainly seemed plausible since most experimental setups for static pressure are unable to detect rapid pressure fluctuations above a certain frequency, hence the hardware provided a naturally damped pressure value.

Exactly how the unsteady behavior influences the onset of bursting was unclear. Pauley's early work [Pauly and Moin, 1990] suggested that a long bubble was steady and a short bubble unsteady. The end of the unsteady behavior marks the beginning of the onset of bursting. This was, however, actually contradicted in later studies by the same authors who concluded that the vortical shedding is actually much stronger than previously thought [Lin and Pauly, 1996]. This conclusion was in part based on Gasters work who documented the significance of the vortical shedding [Gaster, 1966]. In 2000, Spalart and Streets [2000] conducted a DNS study on short laminar separation bubbles and found that an absolute instability was present and the bubble was comparatively small.

It is the interaction between these two mechanisms (convective instability and absolute instability) that may well present and provide an explanation between an abrupt leading 
edge stall and a more gradual thin airfoil stall. In both cases, the unsteady nature of the laminar separation bubble appears to be a crucial point to be taken into account for a proper analysis of the bubble bursting problem.

Nakae et al. [2006] conducted a recent study of the separation bubble on the NACA 0012 airfoil at Reynolds numbers below 100,000. The objective was to actually clarify the relationship between the separation bubble and the aerodynamic characteristics, namely the lift and drag coefficients. Two and three-dimensional time dependent Navier-Stokes equations were solved using a finite difference scheme. No turbulence modeling was invoked. The specific case of 10 degrees angle of attack at a Reynolds number of 100,000 was analyzed. It was found that the separation bubble oscillates in an unsteady fashion, alternating between a short bubble and a long bubble. This agrees with the experimental studies of Mueller and Batill [1982]. As the bubble oscillates, the lift and drag also oscillate in phase with the bubble dynamics. Figure 2.4 shows the results of this study. Since this is a highly unsteady flow, the lift and drag are seen to oscillate in phase with the creation, bursting, and re-creation of the leading edge separation bubble. The frequency of oscillation is about $1.6 \mathrm{~Hz}$. The lift oscillates about $30 \%$ from its mean value and the drag oscillates about $22 \%$ from its mean value. This is the first known study of the actual variation in the lift and drag characteristics with the dynamics of the bubble. 


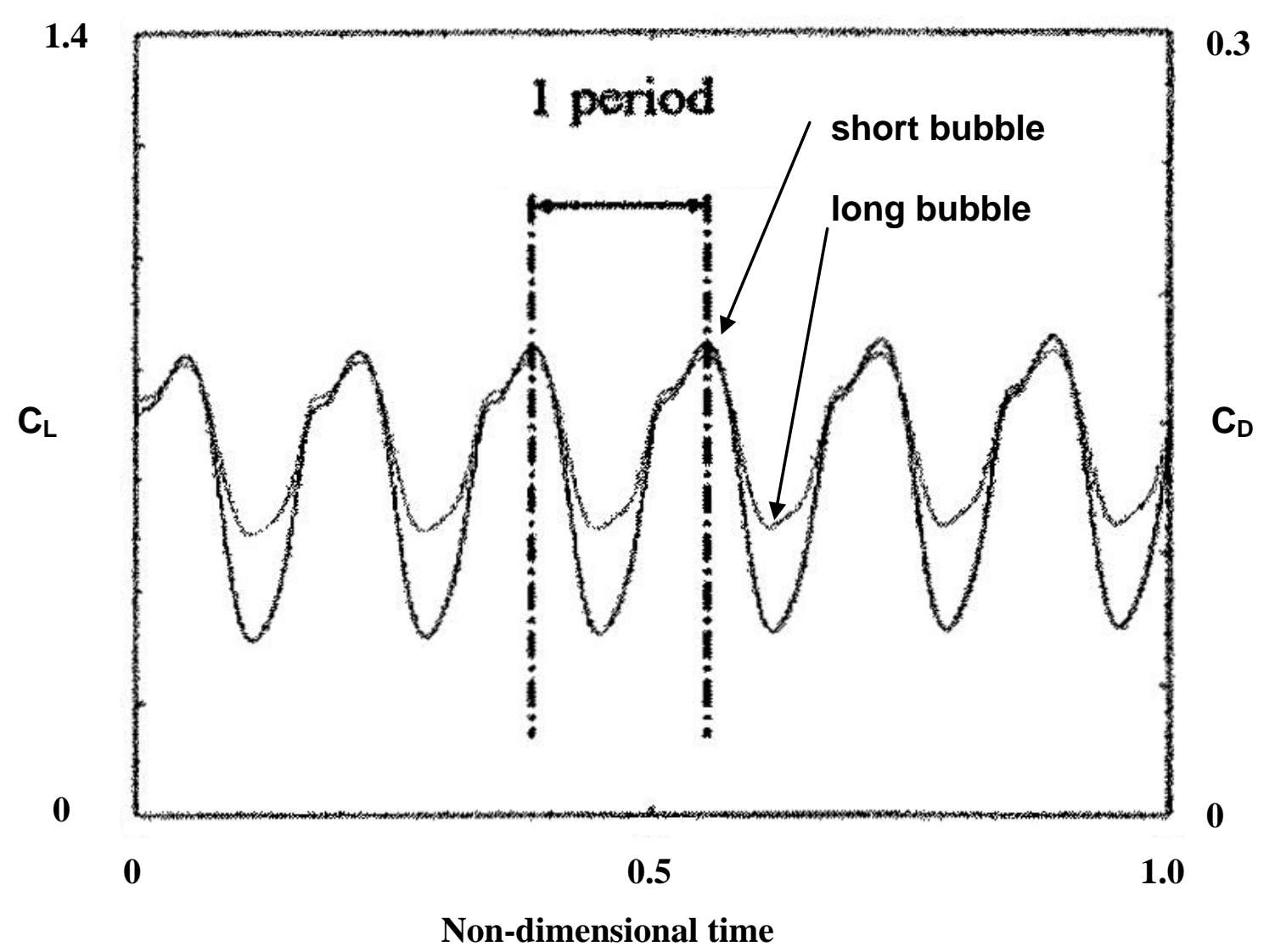

Figure 2.4: Oscillating lift and drag on a NACA 0012 airfoil at 10 degrees angle of attack and Reynolds number of 100,000 [Nakae et al., 2006]

Figure 2.5 is an example of a computational fluid dynamics calculation done in this study showing the laminar separation bubble on a two-dimensional NACA 0012 airfoil at a Reynolds number of 100,000 and an angle of attack of 9.5 degrees using the Fluent ${ }^{\circledR}$ code. This illustration is typical of the analytically predicted short separation bubble. The flow within the bubble is highly unsteady, which is evident from the appearance of primary, secondary, and tertiary vortices. However, of significance is the fact that the separation and reattachment points remain constant. 


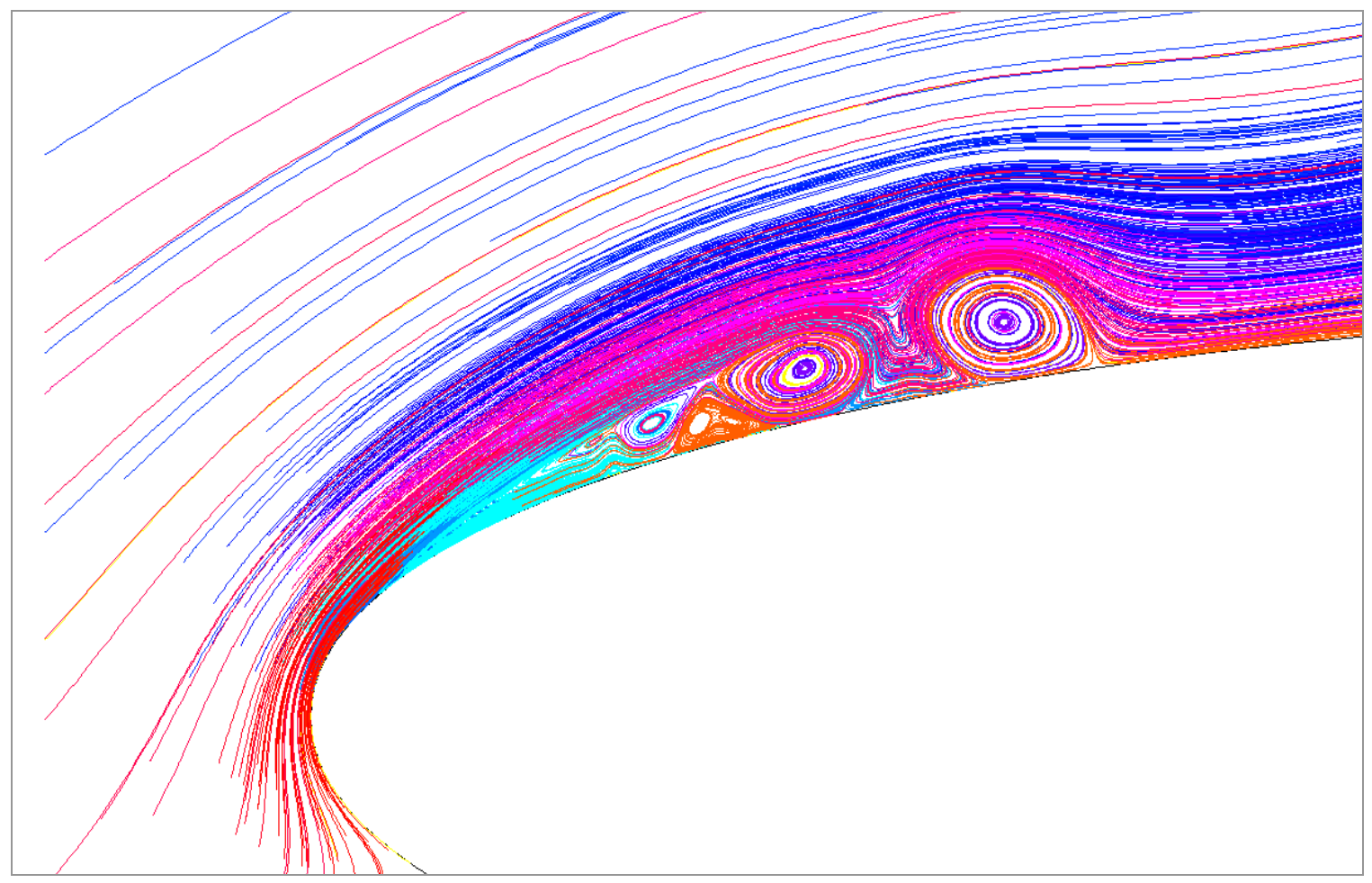

Figure 2.5: Leading edge region of numerical analysis of a two-dimensional separation bubble at 9.5 degrees angle of attack and a Reynolds number of 100,000 (current research)

\subsection{Review of Past Separation Flow Control}

In past research efforts, several concepts and techniques have been studied concerning the improvement of laminar and turbulent boundary layer separation and transition for a given flow field [Greenblatt and Wygnanski, 2000]. These methods have included theoretical and experimental studies as well as numerical approaches to understanding and solving the various flow control problems. Specifically, laminar flow control efforts in the past have included blowing, suction (steady and periodic), compliant moving walls, wall cooling, synthetic jets and plasma injection. Also many methods have been proposed concerning the improvement of turbulent boundary layer flows. A brief history of these 
efforts mainly concerning oscillatory blowing and compliant walls as applied to external flows will now be discussed due to the inherent unsteady nature of these mechanisms and their relevance to the current research effort.

In the early days of aerodynamic flow control, a commonly thought of approach was to use suction and/or blowing (steady and periodic) in order to improve lift and drag performance. Both seemed to have a similar effect on the boundary layer. When applied to a boundary layer that is displaying signs of weak momentum near the wall (i.e. is approaching a state where unless a very favorable pressure gradient is encountered the flow will reverse and separate), blowing tends to replenish the low energy fluid very close the surface with a higher energy stream of air. This tends to increase the momentum of the boundary layer near the wall and prolong separation. Suction on the other hand, takes a different approach. Boundary layer suction leads to a fuller velocity profile and vorticity flux near the wall. Therefore it is commonly used to delay laminar to turbulent transition, postpone separation, achieve an asymptotic turbulent boundary layer (one having a constant momentum thickness), or even re-laminarize an already turbulent boundary layer.

Suction basically inhibits the growth of the boundary layer so that the critical Reynolds number may never be reached. A laminar boundary layer can be maintained until a quite high Reynolds number is reached provided enough suction is provided. There are however, some significant drawbacks to using these systems. The primary concern is the large energy requirement needed to power the systems. As a matter of fact, in many cases 
and applications, more power is actually needed to operate the system (the suction pump) than is actually saved by having the whole system in existence in the first place. A second drawback is the complexity of the required plumbing along with the associated valves, transducers, and regulators [Greenblatt and Wygnanski, 2000]. Also, the additional weight of the systems was a factor.

Nonetheless, these systems have been designed and implemented into production aircraft. The Lockheed F-104 Starfighter featured blown flaps. Lee Persons, a NASA F-104 pilot, exclaimed once to this author "Pete, the 104 is a hot ship, with blown flaps you cross the fence at about 170 knots, loose the blown flaps and you have to increase your speed to about 200 knots, and remember you have no reverse thrust to help slow you down; on top off all that you have to keep the engine spooled up so you have enough bleed to power the blown flaps". Another famous aircraft to use boundary layer control was the McDonnell Douglas F-4 Phantom in the later models. Many claim that this allowed the F-4 to out maneuver the Mig-21, giving it air superiority .It was used on the leading edge and trailing edge devices. It was said to work well, but due to the heavy maintenance of the system it was in many cases "red tagged", meaning temporarily out of service until maintenance could perform work on the system.

Due to high system power requirements, many alternative methods have been proposed in order to minimize the high power requirements. An alternative to this approach was proposed by Greenblatt and Wygnanski [2000]. They proposed periodic or oscillatory blowing commonly referred to as periodic excitation. Their work mainly focused on 
turbulent boundary layer type flows. It was discovered that under certain conditions there exists and range of frequencies at which periodic excitation can be shown to improve flow separation. Interestingly, the flow physics described in their work appears to be similar to the flow physics which have been used in this research when explaining the favorable effects of using dynamic roughness, especially the theories concerning the alteration of the static surface pressure. The most significant finding was that boundary layer entrainment can be altered by relatively small periodic excitations.

Greenblatt and Wygnanski [2000] studied the conceptual bass for periodic excitation. They believed the key feature of the reattachment process is the generation of organized coherent structures in the flow. They observed that the amplifications of the perturbations in the shear layer vary in space and in time during forced reattachment, thinning and stretching at the same time. The emerging vortices attain higher amplitudes as they preserve their strength and travel downstream at late stages in the process. Close to the separation point, the flow is probably absolutely unstable during most of the reattachment process enabling strong temporal amplification of global modes. At the end of the process, the same region is only convectively unstable permitting spatial amplification of the imposed excitation. The global instability may also result in the low initial convection speed of the eddies. It was shown that the forced reattachment of the flow could be caused by enhanced entrainment caused by the passage of the organized vortices. Vortex activity throughout the amplification zone results in net outward transport of mass across the shear layers, an effect which lowers the static pressures on the surface. This ensuing transverse pressure gradient forces the mean flow to reattach to the surface. 
Another area of research which primarily focuses on laminar flow control is that of compliant walls [Bushnell et al., 1977, Rediniotis et al., 2002 and Carpender et al., 2001]. There has been a widespread tendency to use the term compliant to mean any flexible wall. This is not correct. Compliance infers that the flow and the wall properties are in some way matched. Compliant wall is used to describe a passive flexible wall in which the propagation speed of the free surface waves are of the same order of magnitude as the free stream flow. As a matter of fact, research has shown [Carpender et al., 2001] that the best walls for laminar flow control have surface wave speeds that are about $70 \%$ of the free stream velocity. This discussion will focus on compliant walls applied to laminar flow control, that is, the use of compliant walls to postpone or completely suppress transition and separation.

In natural transition, small disturbances are created by several environmental factors, such as free stream turbulence, acoustic vibration, surface roughness, and the entrainment of particulate matter into the boundary layer. The processes by which Tollmien/Schlichting waves are generated through sources of natural excitation are known collectively as "receptivity". Despite their importance, relatively little is known about these mechanisms and how they are affected by wall compliance. After these small waves have been created by the receptivity process they then propagate downstream and grow in amplitude until a point is reached where non linear effects becomes significant. Like a point when a wave on the ocean begins to break. At this point the disturbances become three dimensional, and the transition process rapidly ensues. The actual transition 
zone itself appears as turbulent spots, with the evidence of the Tollmien/Schlichting waves all but gone. However, none of this would have occurred had the Tollmien/Schlichting waves not been present in the first place to begin the process. In low disturbance environments, the two dimensional quasi-linear Tollmien/Schlichting waves represent about 79-80 percent of the total transition process.

The objective of the compliant wall is to greatly extend this linear regime and/or suppress the growth of the Tollmien/Schlichting waves entirely. Several experiments were performed, notably by Gastor [1966]. He found that the route to transition was not gradual through amplification of Tollmien/Schlichting waves, instead it was sudden and occurred when a critical flow speed was reached. It was found that this critical speed was due to "traveling wave flutter" set into the flow from the compliant wall. These instabilities were predicted theoretically based on linear stability theory and could predict the complex response of compliant walls to both Tollmien/Schlichting waves and traveling wave flutter. It became clear at this time that an understanding of the flow induced instabilities, the significance of which was now appreciated, was crucial for designing compliant wall panels for laminar flow control. Basically, passive compliant walls have been shown to work successfully when used in marine type applications. In water flow, the properties required for near optimum compliant walls have been demonstrated to be feasible for manufacture and reasonably practical for many marine applications. But when it comes the aeronautical applications, there are many differences [Carpender et al., 2001]. 
The most obvious is the difference in density, a factor of almost 800 . Another significant difference is that of kinematic viscosity; air is 15 times greater than that of water. When the fluid density is greatly different than that of the wall, there are two main consequences that affect the flow physics. First, the interfacial condition equating the normal surface retraction in the fluid and the solid requires an additional term to account for body force perturbation. This effect, however, is usually minor. Of more significance is the mismatch between the fluid and wall inertias, which comes about when the densities are greatly different. For wall compliance to have a significant effect on Tollmien/Schlichting waves, the wall and fluid inertias much be of the same order of magnitude. This has been verified experimentally [Kramer, 1957]. To match fluid and wall inertias it would be necessary to use a wall material with a density near that of air. Assuming such a material exists (a material like "aerogel') it would need to have a elastic modulus of $3 \mathrm{GPa}$. It is possible to have an elastic modulus in this range for silicone rubber type products, but they are much denser $(1000 \mathrm{~kg} / \mathrm{m})$ than what would be required for these types of applications. Therefore, although compliant walls may currently have a useful application in marine applications, for aeronautical applications their application does not appear feasible at this time. 


\section{Chapter 3}

\section{Flow Physics of Isolated and Distributed Roughness}

The flow physics of leading edge separation and its effects on airfoil performance have been previously discussed in Chapter 2. Since this study also involves roughness elements, specifically dynamic roughness elements, the flow physics of various types of surface roughness will be briefly discussed.

\subsection{Isolated Roughness}

Typical performance specifications for an airfoil assume that the airfoil is smooth and has little or no imperfections. However, when a wing surface becomes exposed to dirt, insects debris, frost, and other surface impurities, small imperfections distributed on the surface can greatly alter the airfoils performance. These imperfections are referred to as surface roughness or just plain roughness. Many research studies [Brumby, 1979 and Valarezo et al., 1993] have been done to examine the various effects surface roughness can have on performance. Studies have shown that roughness located within $5 \%$ chord of the leading edge can have a large impact on airfoil performance. Adding roughness from this point rearward, however, only adds small penalties in overall performance for most airfoil geometries. For this reason, most roughness research on airfoils has been focused on the leading edge region. Roughness affects all areas of airfoil performance, but mainly lift at a given angle of attack, stall angle of attack, and zero lift drag can be affected [Valarezo et al., 1993]. The reduction in maximum lift coefficient can be as much as $20-35 \%$. The increase in zero lift drag can be as much as $15 \%$. 
Understanding roughness and its effects on controlling the state of the boundary layer is important when examining dynamic roughness as a means of flow control. The focus of this discussion is to examine various forms of roughness and the corresponding vortex structures that are spawned by their existence. A given boundary layer control technique can directly modify the shape of the instantaneous or mean velocity profile or selectively influence the small dissipative eddies. Navier-Stokes equations applied at the surface indicate that spanwise and streamwise vorticity fluxes at the wall can be changed, either instantaneously or in the mean by wall motion, streamwise pressure gradients, spanwise pressure gradients, normal viscosity gradients, or a suitable streamwise or spanwise body force [Fasel, 1976]. The vorticity fluxes can determine the fullness of the velocity profile. At this point it is the author's belief that dynamic roughness is a flow control mechanism which alters the state of the boundary layer through unconventional means. Dynamic roughness is not merely another tool used to trip the boundary layer from laminar to turbulent flow in order to keep it attached. If the dynamic roughness was held at its maximum amplitude, emulating a static roughness case, it would not cause the boundary layer to trip.

Braslow et al., [1966] conducted several experiments to determine at what point a particular roughness height would begin to affect the performance of a given airfoil. The roughness height, $\mathrm{k}$, was correlated to the change in airfoil performance by looking at the roughness Reynolds number. The process of transition on an airfoil was believed to be quantitatively similar to the boundary layer transition process on a flat plate. The roughness Reynolds number is defined as the height of the roughness ( the characteristic 
length) times the local velocity at the height of the roughness divided by the kinematic viscosity. It is expressed as follows.

$$
\operatorname{Re}_{k}=\frac{u k}{v}
$$

For a smooth airfoil, $\mathrm{k}=0$. Figure 3.1, taken from Braslow et al. [1966], shows a relationship between the roughness Reynolds number and the zero lift drag coefficient for several aircraft types. As the roughness height is increased the drag remains relatively unchanged until a certain "critical" roughness Reynolds number is reached. At this point, a sudden increase in drag can be observed. This is associated with the premature transition of the boundary layer from a laminar state to a turbulent state. Studies have found this value of roughness Reynolds number to be about 500-600. Relative to this numerical study, this would correlate to a roughness height of about $4.3 \mathrm{~mm}$ on a 1 meter chord airfoil operating at a Reynolds number of 100,000 and a free stream velocity of 1.5 $\mathrm{m} / \mathrm{s}$. 


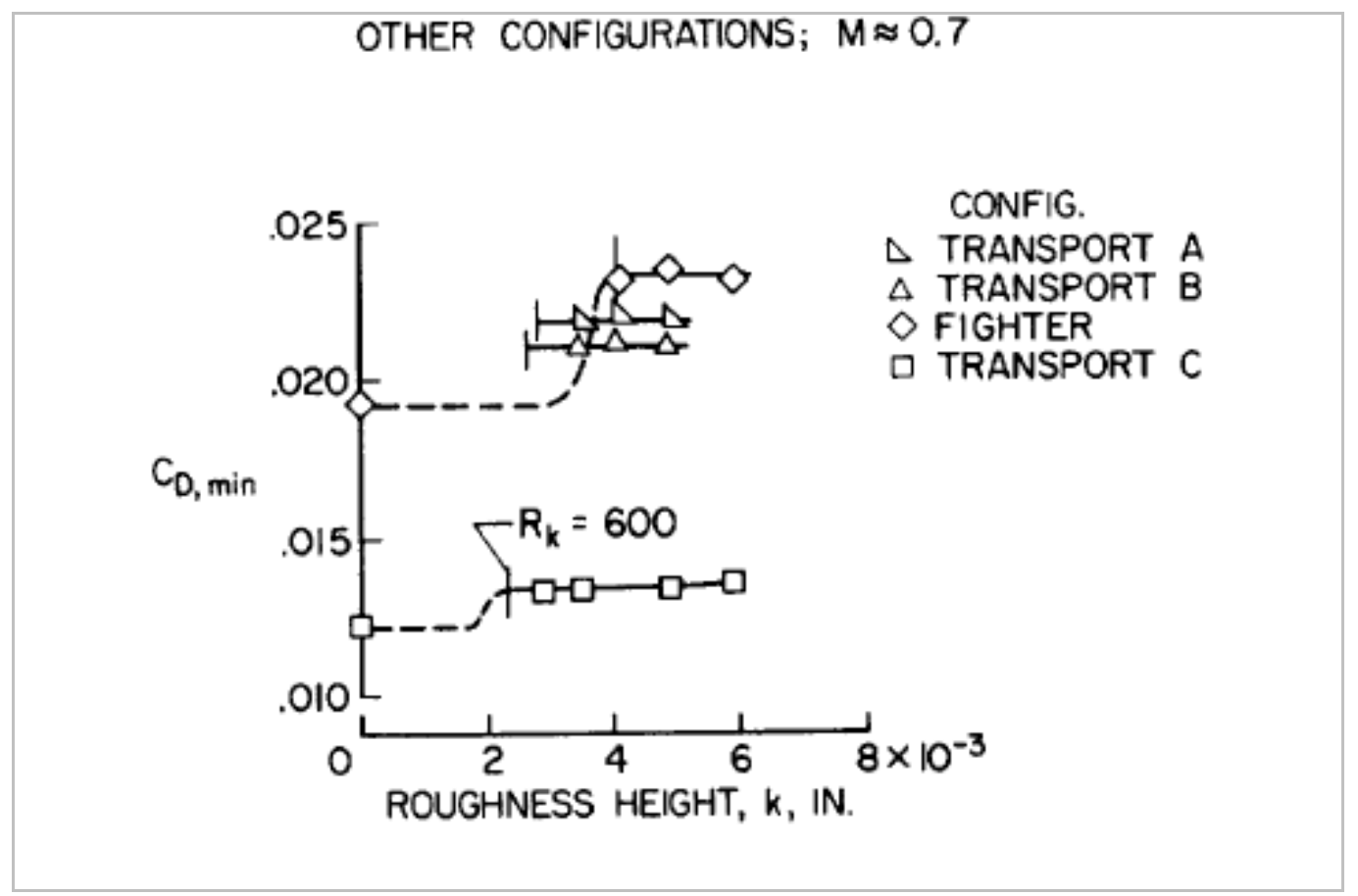

Figure 3.1: Increase in zero lift drag due to surface roughness [Braslow et al., 1966]

The flow physics of the transition process on a clean airfoil is relatively well understood. Once free stream disturbances are ingested into the boundary layer through a receptivity mechanism, they begin to undergo exponential growth which can be described with linearized equations. The initial disturbances can be due to free stream turbulence, vibration, or even sound waves. For airfoils, this growth is referred to as the growth of Tollmien-Schlichting waves. For swept wings, the dominant instability is known as the "cross-flow" mode [Saric et al., 2003]. In either case, once these disturbances have grown sufficiently, a secondary non-linear instability arises which leads to the growth of streamwise vortices [Tani, 1969]. The streamwise vorticity plays a large role in the remaining transition process as the vortices work to redistribute the streamwise momentum and lift the spanwise vorticity from the surface, resulting in a "high shear layer". This "high shear layer" generates hairpin type vortices, which tend to break down 
into smaller hairpin vortices as they convect downstream. This ultimately creates turbulent spots. Eventually the turbulent spots grow and merge until a fully turbulent boundary layer is formed.

When roughness is introduced on the airfoil surface, it can alter the transition process in several ways. The roughness can enhance the growth of the Tollmien-Schlitching waves or introduce new instability modes altogether. Roughness can also enhance the receptivity of the boundary layer, or bypass linear instability mechanisms altogether, acting as a new source of additional disturbances such as vortex shedding. Transition processes which are not initiated by traditional Tollmien-Schlicting waves have been coined "bypass transition" [Morkovin, 1969] . For example, Gregory and Walker [1956] found that a three-dimensional roughness element generates streamwise counter-rotating vortices, which, like the case of clean airfoil transition, tend to convect downstream and generates turbulent spots.

Klebanoff and Tidstrom [1972] studied two-dimensional roughness and found that a characteristic of the flow is a long separation bubble which extends downstream 40-50k from the roughness. $k$ is the height of the roughness element.The velocity profiles within the bubble region are highly inflectional, leading to type a instability known as a Rayleigh instability [Schlichting and Gerston, 2000]. Amplification rates for this type of instability are high, quickly leading to the onset of secondary instabilities. Klebanoff and Tidstrom [1972] confirmed that premature transition due to two-dimensional roughness is 
caused by a large amplification of the Tolmien-Schlichting waves in the separation region behind the roughness.

The mechanism by which three-dimensional roughness enhances transition was studied by Morkovin [1969]. It was discovered that the transition mechanism was not due to significant amplification of Tollmein-Schlichting waves, but rather the formation of coherent vortex structures. The separation region behind three-dimensional roughness is significantly shorter, about 3-6k. As the roughness Reynolds number is increased, a pair of vortices rise from the roughness and turn themselves downstream, creating a pair of counter-rotating streamwise vortices. Additionally, it appeared that a horseshoe vortex is formed just at the foot of the roughness. The legs of this vortex formation convect downstream while staying close to the surface before being dissipated into the wake of the roughness. Figure 3.3 is an illustration of the formation of the coherent vortex structures formed downstream of a three-dimensional roughness element. 


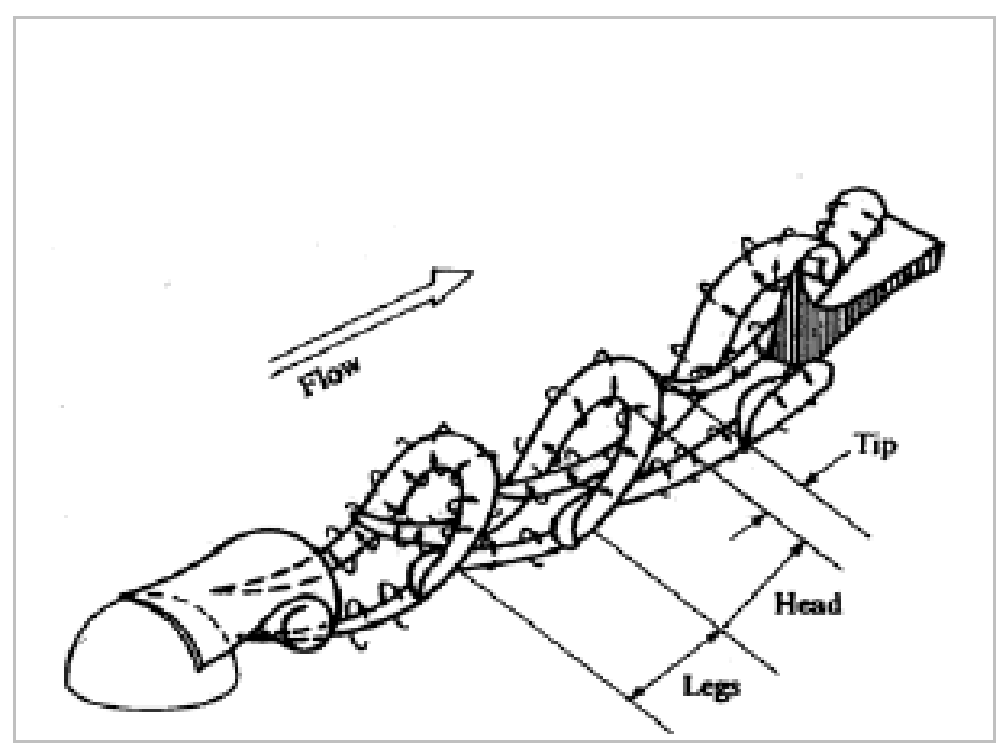

\section{Figure 3.2: Illustration of three-dimensional vortex structure [Acalar and Smith, 1987]}

Acarlar and Smith [1987] studied the vortex shedding characteristics and their relationship to the critical roughness Reynolds number on a flat plate. They discovered that as the $\mathrm{Re}_{\mathrm{k}}$ is increased, a point is reached where vortices are shed periodically into the wake. This was initially believed to occur at about a $\mathrm{Re}_{\mathrm{k}}$ of 400 . Initially the hairpin vortices convect out of the boundary layer without breaking down into secondary structures. Eventually, a value of $\mathrm{Re}_{\mathrm{k}}$ of about 550 is reached and the vortices actually do begin to breakdown. At $\operatorname{Re}_{\mathrm{k}}$ of 600 the transition process begins to take place rapidly and the transition point quickly moves forward to the roughness location. These findings were consistent with the findings of Peterson and Horton [1959] many years earlier except now evidence existed showing the actual creation of the vortex structures. They also studied the shedding frequency as a function of roughness Reynolds number for a variety of three-dimensional geometric shapes. Figure 3.3 is an illustration of the relationship between the roughness Reynolds number and the actual shedding frequency, non- 
dimensionalized in the form of the Strouhal number. In this case the humps were semihemispheric. The roughness Reynolds number is based on the height of the roughness and the Strouhal number is based on the shedding frequency and the velocity at the height of the roughness. There experimental results showed that as the roughness Reynolds number increased so did the shedding frequency.

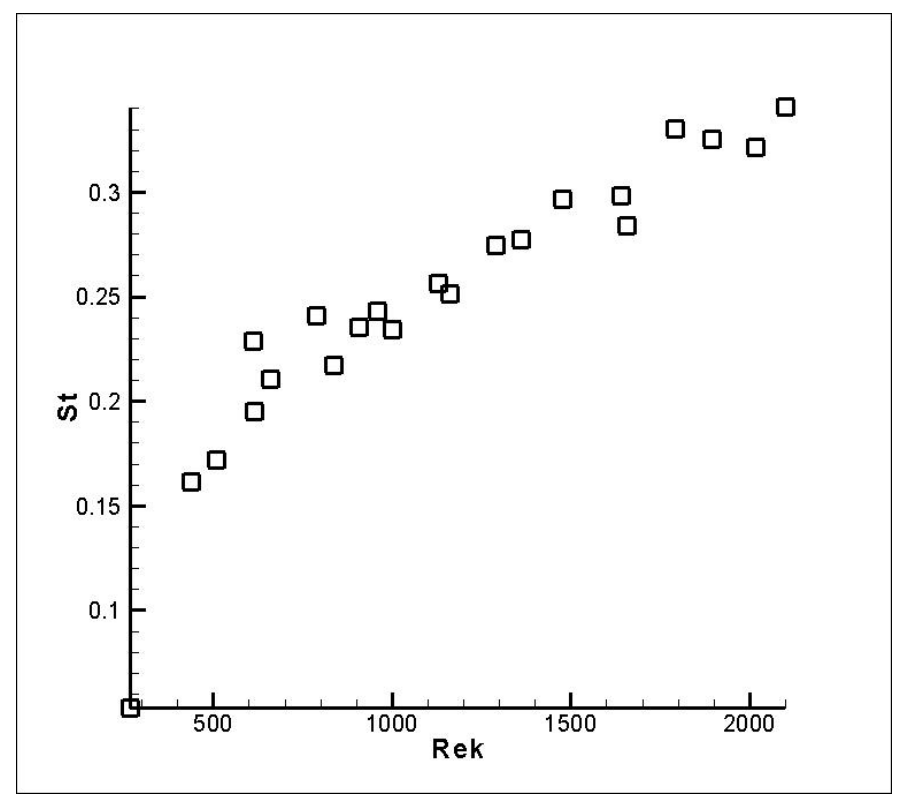

Figure 3.3: Strouhal number as a function of roughness Reynolds number for semihemispheric roughness [digitized from Acarlar and Smith, 1987]

It is interesting to examine whether or not there are differences when comparing transition on a flat plate to the leading edge of an airfoil. A flat plate has a zero pressure gradient where as an airfoil can have a strong pressure gradient, particularity in the leading edge region. Although previous studies indicated that transition occurs at an Rek of 600 [Peterson and Horton, 1959], Bragg [1995] found that the roughness Reynolds number can be as high as 1500 on a NACA 0012 airfoil and the streamwise length 
required for transition can be considerable. This difference seems large, however, upon further examination it was determined that the criterion used for transition was different. Peterson and Horton [1959] used the first observation of non-zero $u_{r m s}$ values where Bragg [1995] used the rapid approach of the transition location to the roughness element itself. This was the same method used by Tani [1961]. Figure 3.4 is a plot depicting the effect roughness Reynolds number can have on the boundary layer as a function of the arc length distance from the leading edge. This work showed that the unsteady vortex shedding from the roughness element corresponds well with the critical roughness Reynolds number for transition.

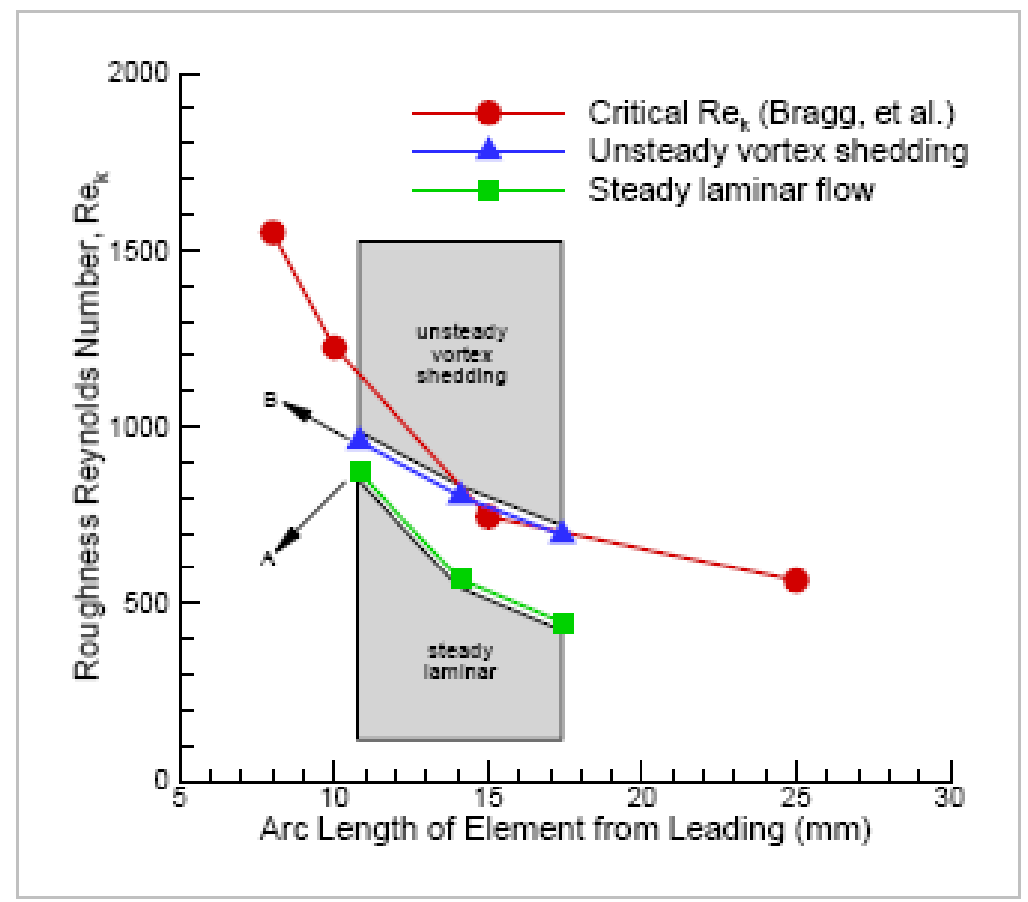

Figure 3.4: Effect of critical Reynolds number on flow [Matheis et al., 2004] 


\subsection{Distributed Roughness}

Unfortunately, the process of transition due to distributed roughness is even less understood than the isolated roughness cases. Morkovin [1969] suggests that distributed roughness may increase receptivity, cause amalgamation of streamwise vorticity leading to a new linear instability, and increase the overall disturbance level by direct eddy shedding. Studies have shown, not surprisingly, that the transition due to distributed roughness is not due to Tollmein-Schlichting wave amplification, but again, some type of bypass transition. Braslow showed that distributed roughness behaves similarly to isolated roughness. Near the leading edge there is an increase in the $\mathrm{Re}_{\mathrm{k}}$ value which is identified as the $\mathrm{Re}_{\mathrm{x}}$ effect. Further downstream, once a certain value of $\mathrm{Re}_{\mathrm{x}}$ is reached, $\mathrm{Re}_{\mathrm{k}}$ approaches a constant value of about 600. Kerho [1995] and Kerho and Bragg [1990] observed three types of transition mechanisms. One was a linear growth of $u_{\mathrm{rms}}$

values just downstream of the roughness followed by an asymptotic approach to a fully turbulent boundary layer. The second is a short delay followed by the growth of $\mathrm{u}_{\mathrm{rms}}$ values and again an asymptotic approach to turbulence. The third was the growth of Tollmein-Schlichting waves.

\subsection{Conceptual Basis}

Numerous studies have examined the use of a movable or flexible wall [Bushnell et al., 1977, Rediniotis et al., 2002 and Carpender et al., 2001]. In these studies, the movable surface is viewed as a mechanism which can introduce disturbances into the flow. It is widely viewed that coherent motion when subjected to excitation can create large quasideterministic, vertical spanwise structures which essentially act as "building blocks" in 
the mixing layer. These are responsible for the momentum transfer across the extent of the mixing layer. Hence, entrainment can be significantly altered by very small amplitude excitation when applied near the origin of the shear layer [Greenblatt and Wygnanski, 2000].

Using a two-dimensional Navier-Stokes solver, Huebsch, [2006] showed that by applying dynamic roughness the leading edge separation bubble could be entirely eliminated and the downstream vortex shedding created by the separation bubble could be minimized. A similar result may be seen when analyzing the details of the flow within the boundary layer. The work of Huebsch, [2006] showed that the flow about the leading edge changes to an attached flow when the dynamic roughness is turned on, without any significant large scale unsteadiness in the flow. In fact, the same work showed that the only unsteadiness in the flow is a small scale unsteady separation located right at the dynamic roughness elements, which remained sub-boundary layer. There was no evidence of large scale unsteady separation, significant flow instabilities or transition elsewhere in the flow. This means that the small scale unsteady surface roughness was completely altering the state of the flow along the entire leading edge. The leading edge becomes attached and laminar when the dynamic roughness was turned on, whereas it was highly separated with significant unsteadiness when the dynamic roughness was turned off.

As noted above, this result is perhaps to be expected. Dynamic roughness which takes up a fraction of the boundary layer will completely alter the local state of the 
boundary layer over the entire region where the dynamic roughness is located. In effect, the dynamic roughness is creating its own local flow field which, in principle, will induce Reynolds stresses within the boundary layer. The author believes that it is possible that these Reynolds stresses are acting in such a way as to accelerate the flow, thereby avoiding separation. It should be noted that other unsteady three-dimensional effects, such as the creation of hairpin eddies about the dynamic roughness, could also act to energize the boundary layer. 


\section{Chapter 4}

\section{CFD Set-up for Dynamic Roughness}

A computational fluid dynamics numerical study was carried out using the GAMBIT ${ }^{\circledR}$ and Fluent ${ }^{\circledR}$ commercial software platforms. Gambit 2.4.6 and two versions of Fluent ${ }^{\circledR}, 6.3 .26$ and 12.1 were used. Version 12.1 was introduced during the last year of this research effort and is the most recent version of Fluent ${ }^{\circledR}$. It incorporated many user friendly updates. Calculations were carried out using a parallel processing approach in which the domain is divided into several smaller domains each with about the same number of cell volumes such that calculations could be carried out simultaneously. The Beowulf Linux cluster (NIFTY) consisted of 6 nodes, with two processors and 8 core nodes each. Each core processor has access to $2 \mathrm{~GB}$ of RAM . In this study, 8 core processors were used in parallel.

For the pre-processing phase, both two-dimensional and three-dimensional modeling was done by incorporating the GAMBIT ${ }^{\circledR}$ pre-processor. This is a pre-processing platform which allows the user to create various geometries such as an airfoil or wing and its corresponding mesh for main solver input. For the solver phase, Fluent was used to solve the continuity and momentum equations (Navier-Stokes). Several user defined function algorithms (UDF's) were developed that simulated the motion of the dynamic roughness. Acceptable grid remeshing proved to be a tedious process. A combination of several parameters in the layering, smoothing and remeshing algorithms required extensive tailoring in order to obtain a mesh which would 
properly readapt to the moving dynamic roughness. Post-processing was done using TECPLOT, a versatile graphics and plotting software package.

\subsection{Problem Set-up}

In the first phase of this study, a two-dimensional model of the NACA airfoil was modeled in the GAMBIT ${ }^{\circledR}$ pre-processor program. The airfoil coordinates were created using the equations in Abbot and Von Doenhoff [1956]. In the second numerical phase of this study a three-dimensional finite wing was modeled using similar techniques.

For both the two-dimensional and three-dimensional models a C-type computational grid was constructed. The grid for both models extends three chord lengths upstream, four chord lengths downstream and four chord lengths above and below the wing. Although it is common for the far field region of this type of flow field to extend beyond these ranges, these values were chosen to be adequate since the emphasis of this research was the boundary layer flow physics near the surface and not global aerodynamic parameters.

Once the domain was established, an unstructured mesh was carefully constructed using a combination of rectangular, quadrilateral, tetrahedral and triangular type cells in a manner which would allow good resolution of the flow physics near the surface while at the same time managing the overall cell count. Specific zones on the surface of the airfoil were identified and marked to be a compliant type surface in which the motion was governed by its corresponding user defined function (UDF). When applying moving walls, it is a requirement of the code that the mesh consist of triangular (two- 
dimensional) or tetrahedron (three-dimensional) type sells in order for the remeshing algorithms to function correctly.

For the two-dimensional study, the basic airfoil was modeled to have a one meter chord length and a thickness ratio of $12 \%$ (NACA 0012). The dynamic roughness initially consisted of a series of 14 humps placed in fourteen zones beginning at the $0.6 \%$ chord location and extending to the $3.2 \%$ chord location. The first location was chosen based on the fact that the normal separation point for this airfoil application is downstream of the $1 \%$ chord location. This would allow the roughness field to be located just upstream of the normal laminar separation point. The chordwise length of each zone was $2 \mathrm{~mm}(0.2 \%$ chord) which corresponds to the wavelength of each individual roughness element. The hump geometry was initially shaped like a rotated axisymmetric sine wave function and had a maximum amplitude of $0.8 \mathrm{~mm}(0.08 \%$ chord $)$ and a total wavelength of $2 \mathrm{~mm}$ $(0.2 \%$ chord $)$. The frequency and amplitude of each individual row of humps could be changed by adjusting parameters in the UDF. These initial analysis values were chosen based on previous research [Huebsch, 2006] which indicated that the roughness height is most effective when its amplitude is approximately $50-80 \%$ of the oncoming boundary layer height. The boundary layer in the region of the first hump is approximately $1.4 \mathrm{~mm}$ $(0.14 \%$ chord $)$ in thickness. This was determined by computational fluid dynamics numerical analysis and checked with classical Blasius laminar boundary layer theory, both of which agree reasonably well. 
Figure 4.1(a) shows the overall two-dimensional grid, while figure 4.1(b) is a closer view of the region near the airfoil. Several zones around the airfoil can be observed. This facilitates close control of the unstructured meshes in order to create the minimum amount of cell volumes while at the same time capturing the flow physics. Figure 4(c) is a closer view of the hump region. A dense number of cell volumes in this region can be observed. The hybrid grid can also be observed (structured mesh in the outer domain). This was done to optimize the amount of cell volumes used in order to increase run times.

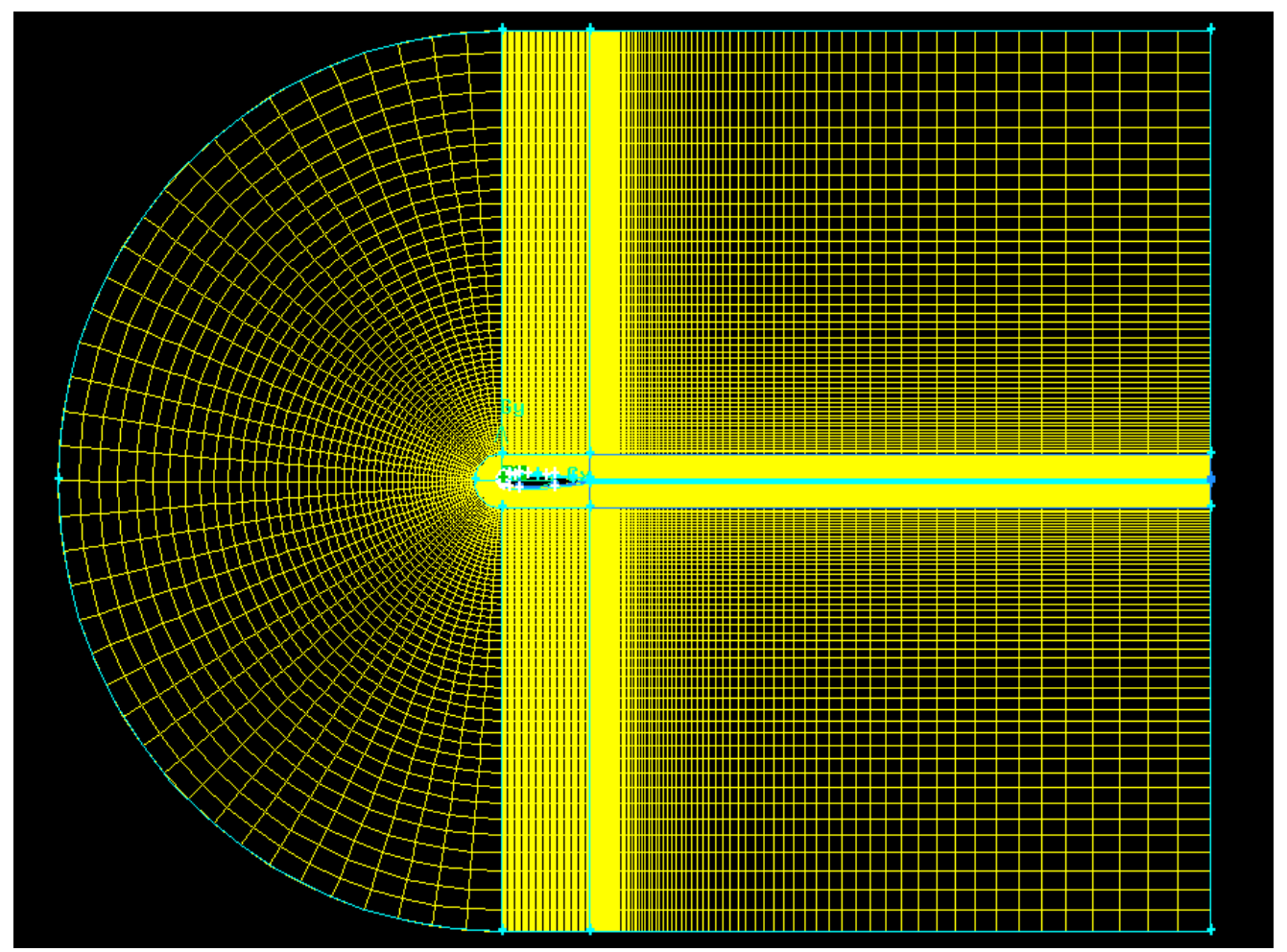

a) Two-dimensional hybrid C-type grid 


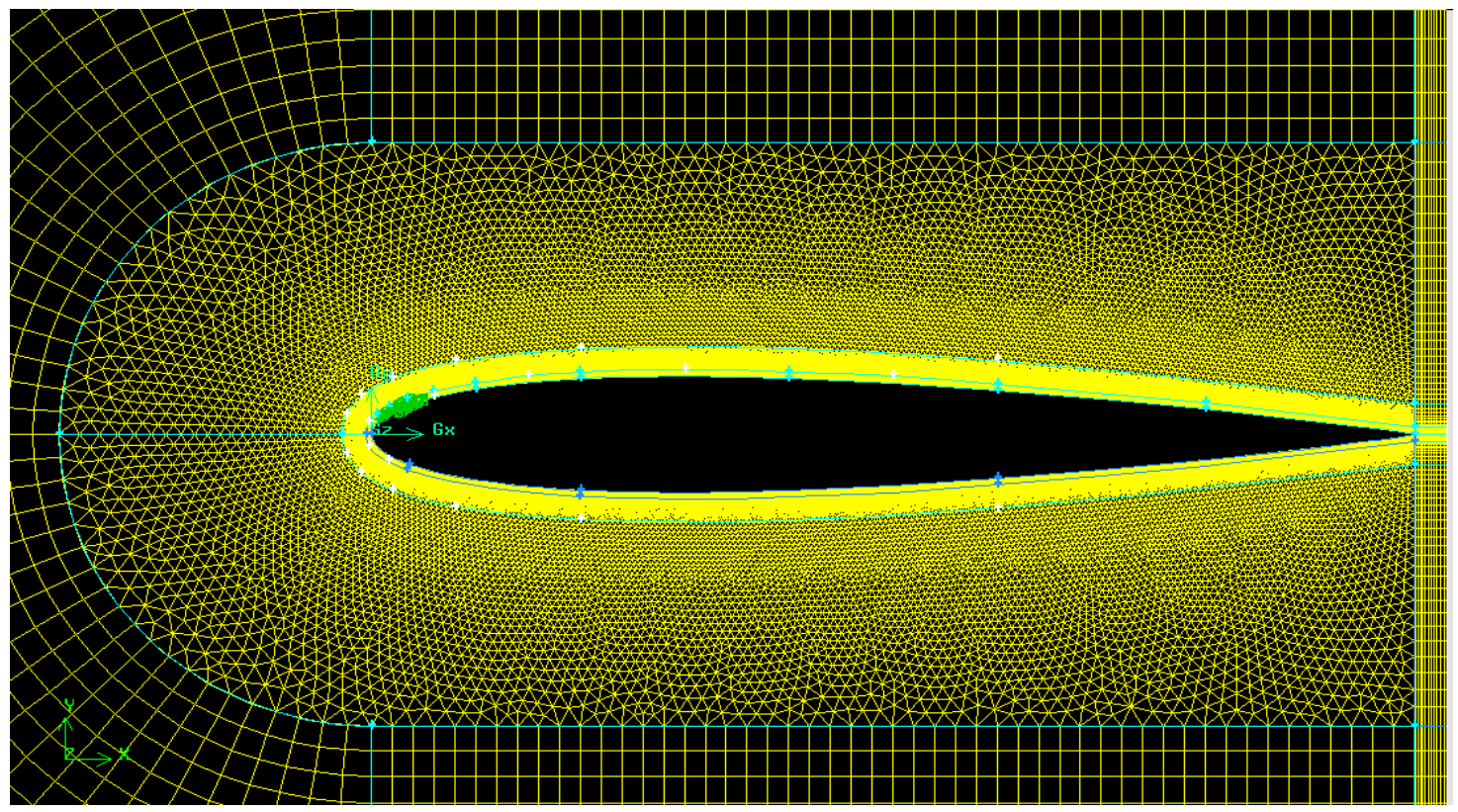

b) Close-up of two-dimensional grid depicting several zones near the airfoil

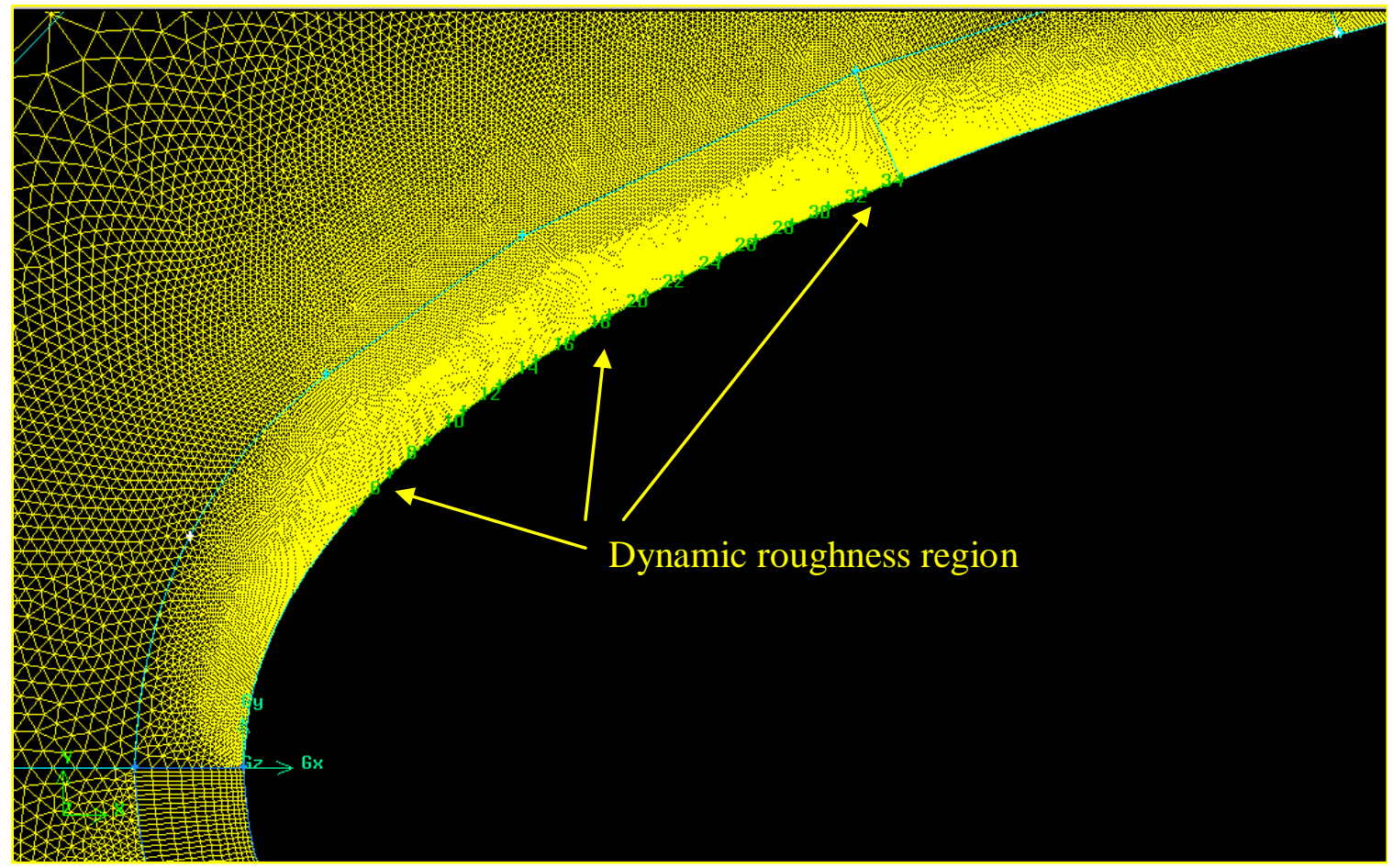

c) Dynamic roughness region of two-dimensional grid

Figure 4.1: Two-dimensional grid images 
To evaluate three-dimensional flow physics, a three-dimensional model of the NACA 0012 airfoil was modeled in Fluent ${ }^{\circledR}$ similar to the two-dimensional case except for a finite span of $50 \mathrm{~mm}$ (5.0\% chord). Several models were developed which could model dynamic roughness ranging from the $0.6 \%$ chord location to the $5.0 \%$ chord location. As in the two-dimensional case, the first location was chosen based on the fact that the normal separation point for this airfoil application is downstream of the $1.0 \%$ chord location. This would allow the roughness field to be located just upstream of the normal laminar separation point. Three-dimensional zones were constructed which served as compliant surfaces which in turn were governed by their respective UDF's. Several UDF's were written which simulated axisymmetric humps as well as spanwise ridges with varying amplitudes and frequencies, depending on the desired governing motion. Figure 4.2 depicts a portion of the three-dimensional grid. Figure 4.2(a) illustrates the finite span and the region where the dynamic roughness was located. Figure 4.2(b) illustrates the zone constructed in the region where the roughness field is located. It was in this region that the highest concentration of cells were used. Analysis of results focused on the central region of the span. Figure 4.3 depicts a plane slice through the roughness region. In this figure, the humps are flush with the airfoil surface. The various colors indicate the skewness of the cell volumes. Pink depicts the highest skewness and blue depicts the lowest skewness. 


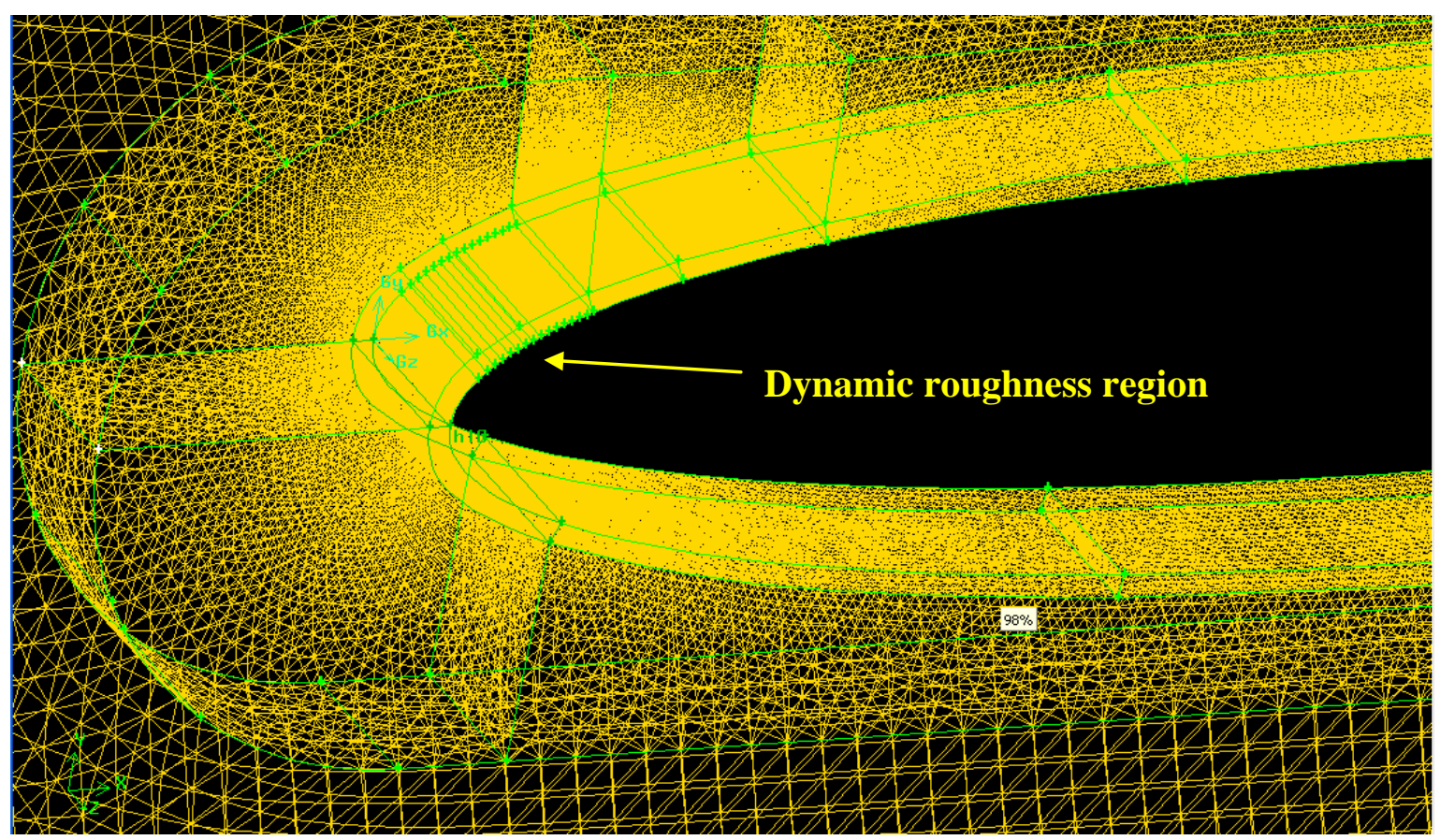

a) Portion of three-dimensional grid

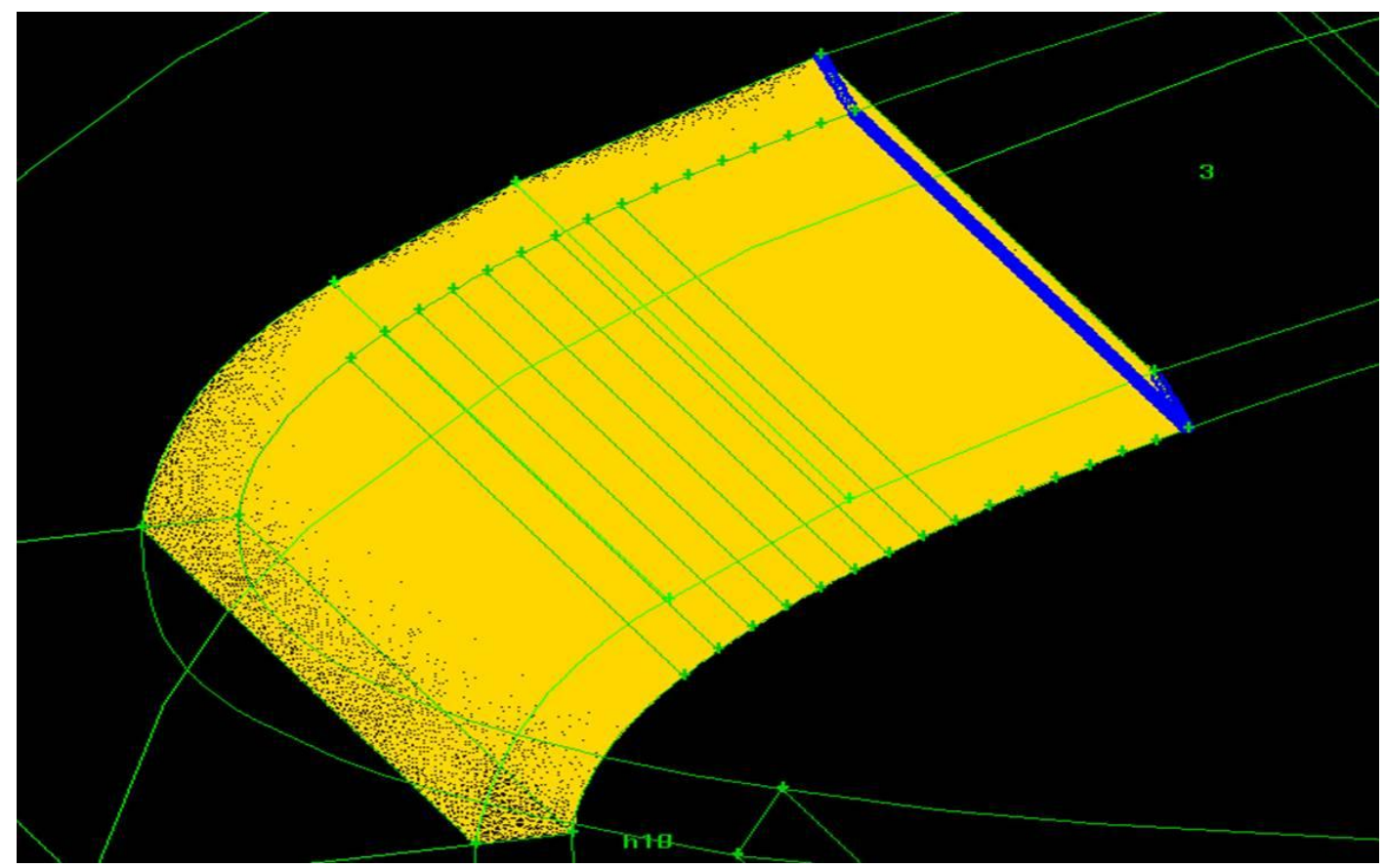

b) Close-up view of grid within three-dimensional dynamic roughness region

Figure 4.2: Portion of three-dimensional grid 


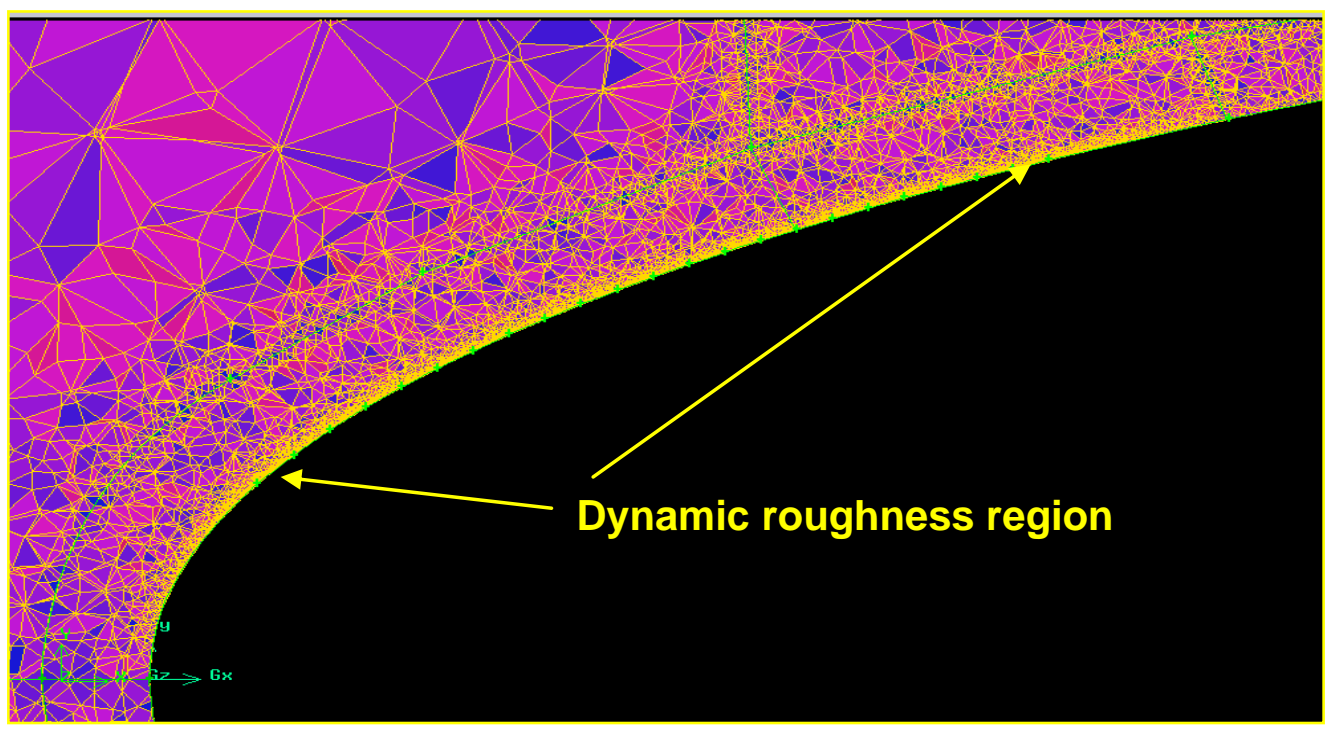

Figure 4.3: Planer cut of three-dimensional dynamic roughness mesh

After defining the compliant wall zones, the mesh had to be carefully created. As previously noted, tetrahedrons must be used when applying moving wall motions.

In the region of the dynamic roughness, the grid contained approximately 20 nodes in the chordwise direction over each hump. This same spacing was used in the spanwise direction for the three-dimensional model. This yielded a characteristic cell length of 0.1 $\mathrm{mm}$ of $0.01 \%$ of the chord length. Since the computational work was primarily focused on the leading edge region and the separation point, a global grid independence check was not conducted. However, the local grid region surrounding the dynamic roughness was refined to ensure that the simulations properly captured the effects on the boundary layer. The grid resolution of 20 nodes over each roughness element in both the chordwise and spanwise directions showed grid independence for the local flow field. It should be noted that Huebsch [2006] conducted extensive grid independence studies for two- 
dimensional dynamic roughness and found similar grid density requirements to properly capture the local boundary layer. The global grid was capable of predicting the correct angle of attack and separation location for a clean wing as compared to experimental work. The final overall mesh required extensive run times to properly simulate the unsteady flow results and acquire the flow control results. Typical run times were several days for a single case using eight processors

Successive grid spacing was held to a maximum growth factor of 1.2. Figure 4.4 is a shaded view of two of the types of surfaces that were studied. One case represents axisymmetric humps and the other case represents spanwise ridges. The spanwise ridges taper in a sinusoidal fashion to flush with the airfoil surface near the tip of the span. The chordwise length of the roughness region is the same for both of the hump and ridge geometries.

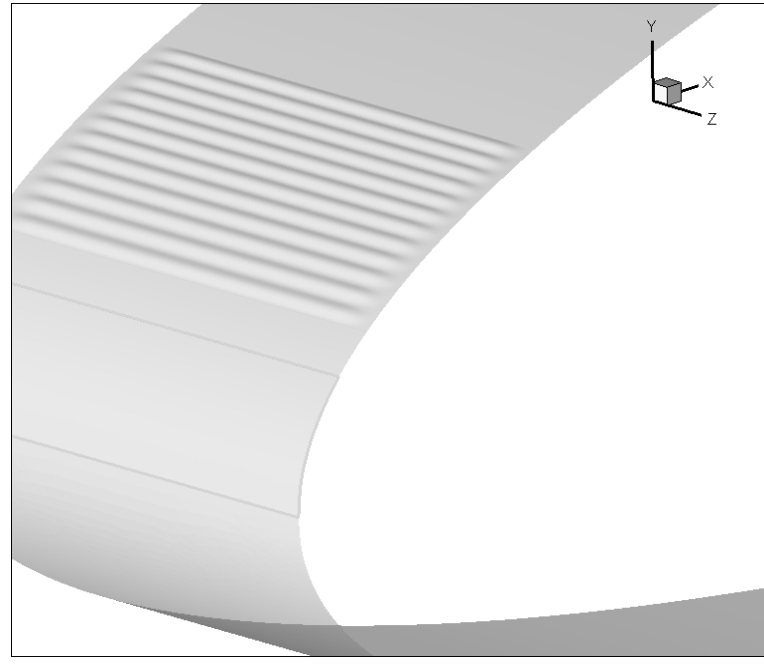

a) Twelve rows of ridges

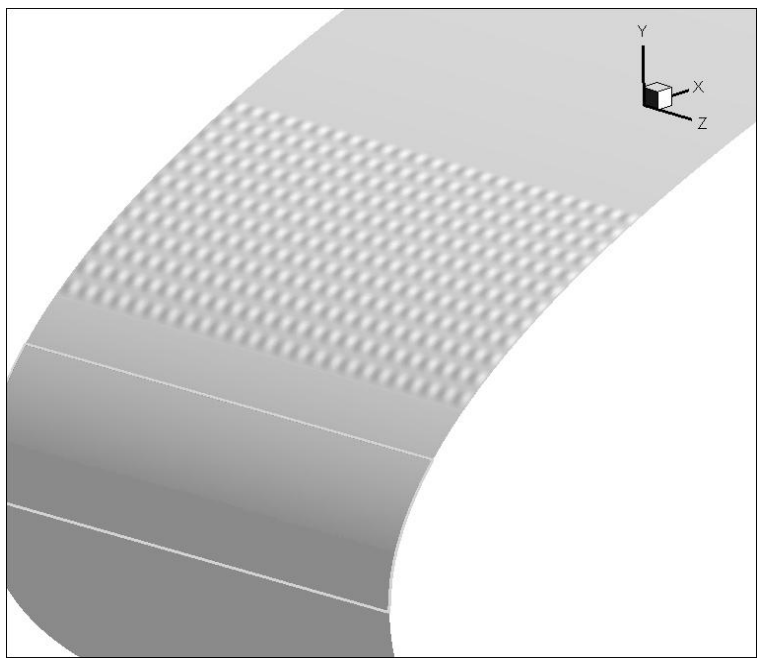

b) Twelve rows of humps

Figure 4.4: Surface depiction of surface humps and ridges on airfoil leading edge 
Figure 4.5 is a close up of the hump region. The faceted faces of the hump which identify the location of the tetrahedron cells can be observed. The illustration may appear to be skewed somewhat due to the perspective used in the graphics module, however in the actual model the hump is symmetrical.

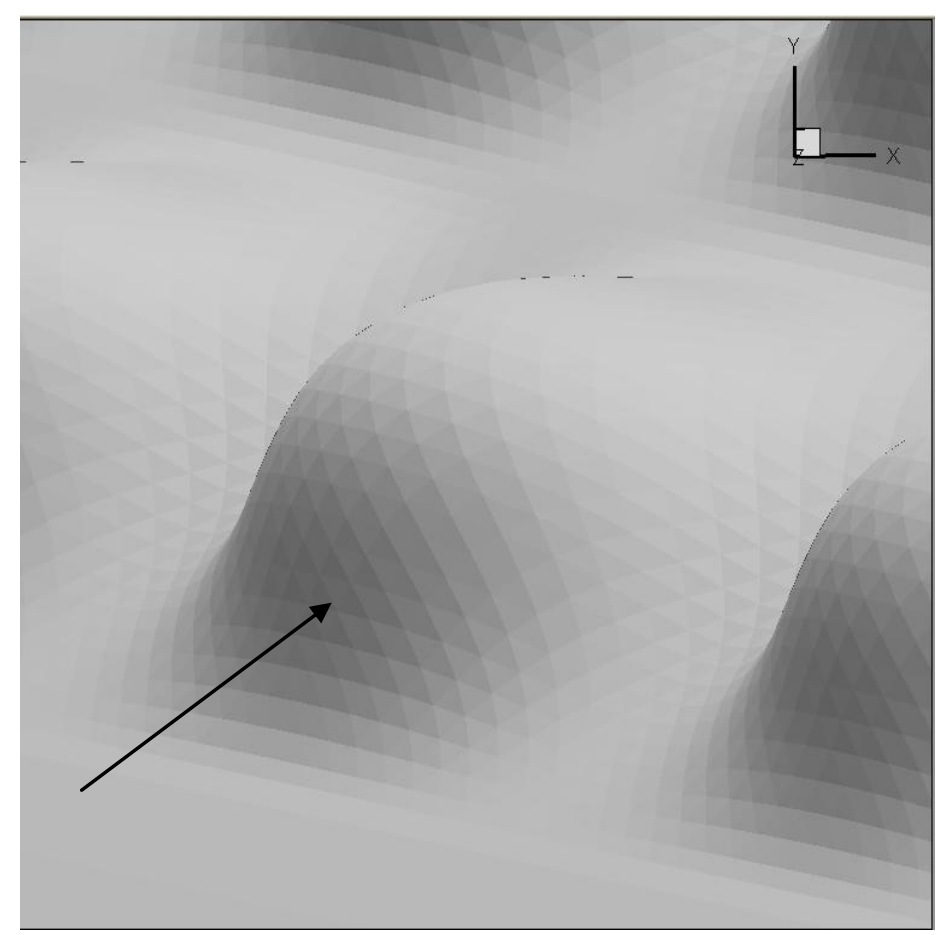

Figure 4.5: Close-up of hump region

\subsection{Deforming Grid Generation}

Many difficulties were encountered in developing a dynamic mesh which would remesh with each hump movement in an acceptable manner. This required several adjustments to input algorithms which govern the remeshing capabilities of the code. A combination of several parameters in the smoothing and remeshing algorithms required extensive tailoring in order to obtain a mesh which would properly readapt itself to the moving surface. There are three algorithms, namely layering, smoothing, and remeshing [Fluent, 
2009]. It is imperative to have an understanding of these three algorithms in order to establish a grid which can properly reform after each successive time step.

Layering involves the creation and destruction of cells. Cells are added or deleted as the cell zone grows and shrinks. As cells are added and deleted their connectivity changes. It is available and works well for quad, hex, and wedge shaped cells and volumes. Layering is most useful for a linear type motion but can be used for pure rotational motion as well. Examples are a piston moving inside a cylinder or a door opening and closing. There are several options to choose when layering is used. A constant height option can be used, which maintains every cell layer at a constant height. Also, a constant ratio option can be used which maintains a constant ratio of cell heights between linear growth layers. This is useful when layering is done over a curved surface. Layers can also be added or deleted based on the current layer height and ideal layer height by setting up a split factor and collapse factor. This basically allows cells to be collapsed and split based on certain user inputs. The ideal height is typically defined as a parameter by the user, but it is usually the same as a typical cell height in the model.

There are some limitations imposed when using layering. You must have a one-to-one interface, and the dynamic mesh does not allow for a topology change; that is at least one layer of cells must always remain in a given dynamic zone. Also, it is only available for quad, hex, and wedge type cells. In addition, layering can produce skewed cells if the face is not normal with the extrusion direction. This would be a problem for highly 
curved surfaces such as those encountered in this research effort. Therefore, the layering algorithm was not used.

Smoothing is the most common form of remeshing where the nodes move as if they are connected with springs or as if they were part of a sponge. The connectivity remains unchanged. It is limited to relatively small deformations when used as a stand alone meshing scheme. It also works best when applied to triangular and tetrahedral meshes, although it may also be used with quad, hex, and wedge mesh elements with a special command input. Examples of applications are arterial walls and membranes. Smoothing is also well suited for the dynamic roughness application. Basically, the iterative smoothing algorithm is controlled via the convergence tolerance and number of iteration inputs. Boundary zone nodes are typically held stationary unless defined otherwise as a dynamic zone. Another parameter is the boundary node relaxation. This allows the boundary nodes to relax to accommodate the remeshing of the interior nodes. Smoothing, like layering, does not guarantee optimum cell skewness. Remeshing is often needed in conjunction with smoothing in order to obtain a proper mesh. It is very useful for small motion as a function of time.

The remeshing algorithm is often utilized when the motions of the moving boundaries are significant relative to the grid [Fluent, 2009]. Translation and rotation may be involved. Cells and faces are remeshed when skewness and/or size exceeds limits set by the user. As cells and faces are added or deleted, connectivity usually changes. It is available for triangular and tetrahedral type elements. For some applications, such as this one, only 
interior cells need to be remeshed. This is referred to as volume remeshing. Along with volume remeshing there are three types of face remeshing available: region face remeshing, local face remeshing, and $2.5 \mathrm{~d}$ face remeshing. For this application only volume remeshing was used. When remeshing, all cells with skewness above a certain limit are marked. Also all cells which are above a "maximum length scale" or below a "minimum length scale" are marked. Local remeshing only operates on the marked cells. Maximum cell skewness is usually used to mark cells. Once the wall has moved, the skewness of the cells usually increases. Therefore, there is no direct control over the skewness of the remeshed cells, they can only be controlled indirectly. Remeshing occurs at the beginning of the time step, before the moving boundaries are actually moved. Interpolation occurs after remeshing.

Figure 4.6 depicts an early attempt at remeshing where a poor mesh was created. In figure 4.6(a) the parameters of the remesh zone were too restrictive, which did not allow the cells to compress adequately throughout the expansion of the humps. In figure 4.6(b) the remeshing parameters did not allow the cells to rebuild themselves during the contraction phase of the cycle. After several adjustments, successful remeshing was accomplished as can be observed in figure 4.7. The smoothing and remeshing algorithms wee utilized to accomplish this dynamic mesh motion. 


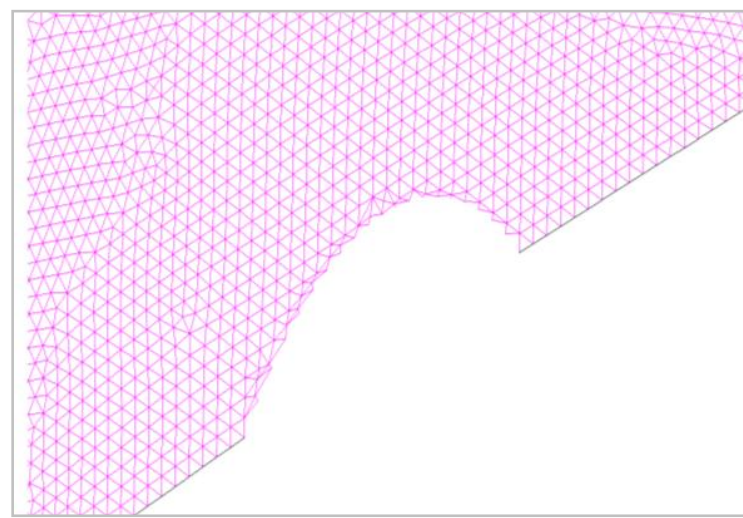

a) Unacceptable expansion of hump

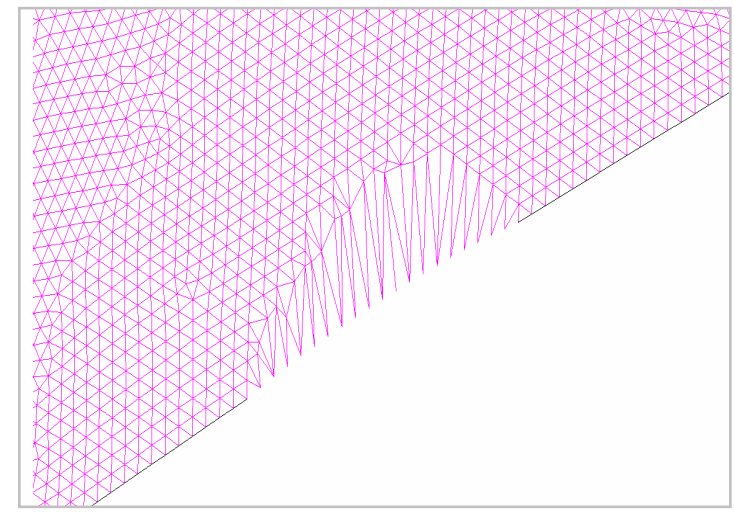

b) Unacceptable contraction of hump

Figure 4.6: Improper grid remeshing

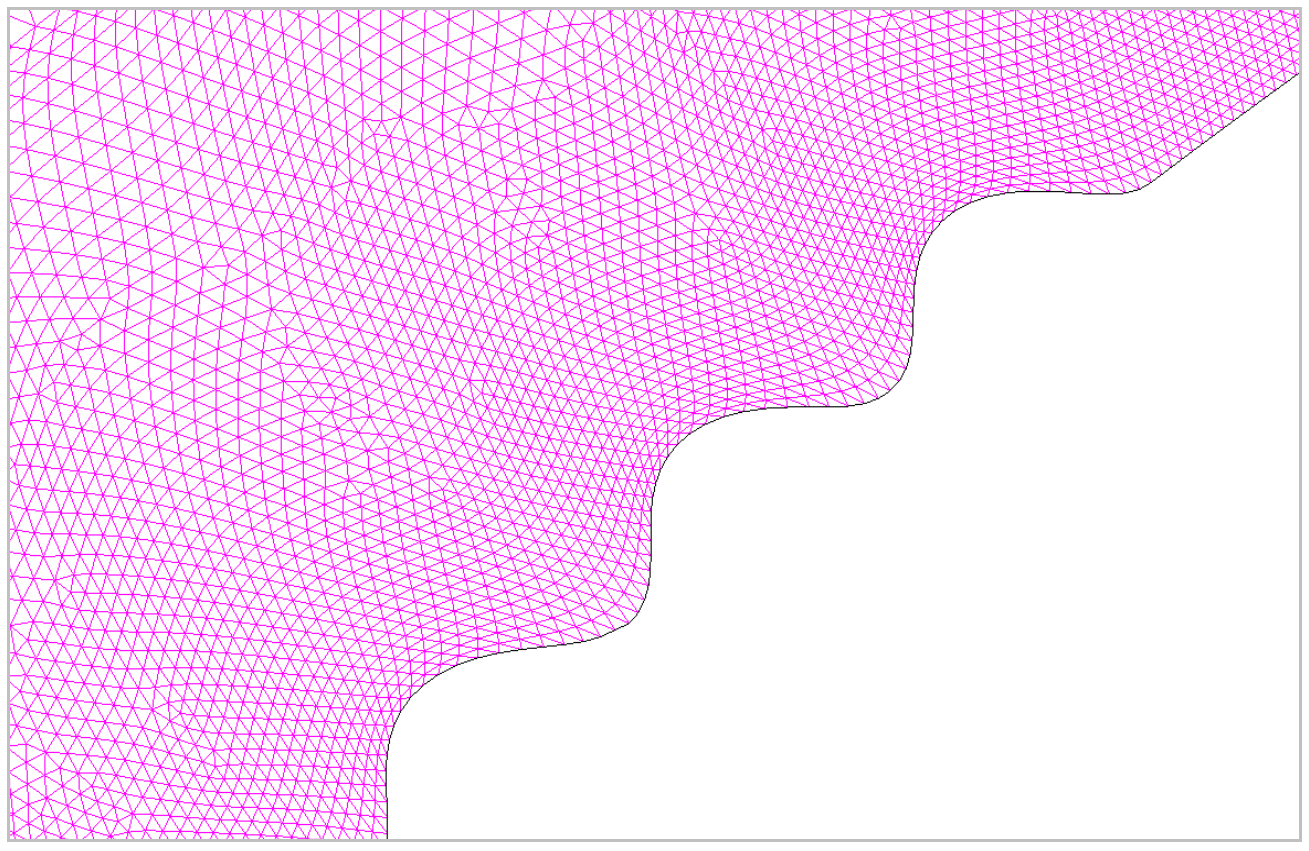

Figure 4.7: Acceptable grid remeshing in roughness region

UDFs were required to achieve the dynamic mesh motion for the dynamic roughness.

The UDF had to be written in a language which is a combination of $\mathrm{C}$ and Fluent's own 
unique user commands. Appendix A lists a UDF which was successfully used in three dimensional cases. Also, in the three-dimensional cases, two roughness models were studied, one with 12 humps extending from $0.8 \%$ to $3.2 \%$ chordwise location and a second model with 20 humps extending from $0.6 \%$ to $5.0 \%$ chordwise location. The dynamic roughness was modeled in both cases as spanwise ridges or spanwise humps. Table 4.1 is a list of the various parameters along with the values that were used to provide acceptable grid remeshing.

Table 4.1: Grid smoothing and remeshing parameters

\begin{tabular}{|l|l|}
\hline Connectivity spring constant & 0.00001 \\
\hline Remesh interval & 1 \\
\hline Convergence ratio & 0.10 \\
\hline Minimum length scale & 0.213 \\
\hline Maximum length scale & 0.000045 \\
\hline Remesh minimum length scale & 0.200 \\
\hline Remesh maximum length scale & 0.000054 \\
\hline Remesh iterations & 10 \\
\hline
\end{tabular}

\subsection{Boundary Conditions and Solver Setup}

The basic code (Fluent) is a finite volume implicit solver which solves the continuity and $\mathrm{x}, \mathrm{y}$ and $\mathrm{z}$ momentum equations utilizing an implicit solver for compressible and 
incompressible flows. In this study a laminar flow analysis was used. The reasons for this are as follows: 1) the separation bubble is typically a laminar flow phenomenon 2) the leading edge region of most airfoils is primarily a laminar flow region and 3) if we continue to increase the resolution of the flow domain in this region, we eventually reach a point where the flow approaches the threshold of DNS modeling, depending on the Reynolds number and smallest turbulence length scale, which inherently requires no turbulence modeling. Therefore, this flow type was selected and used throughout the entire flow domain except for the examination of some special cases.

There were some cases run with a turbulence model downstream of the roughness region where natural transition would normally take place. This was done to examine the effect of turbulence and transition once the flow has passed over the roughness region. This mainly was accomplished to see if turbulence modeling would suppress the formation of the downstream vortex formation, which seemed to be an artifact of using a laminar solver [Huebsch, 2006]. In order to study this, a separate fluid zone must be established in the approximate area of interest since the exact transition location is not a known priori.

When a turbulence model was applied to the entire flow domain, the leading edge laminar separation bubble would cease to exist for the specific cases analyzed (9.5 and 12 degrees angle of attack at a Reynolds number of 100,000 and 150,000). Results indicated that this application did indeed suppress the formation of the downstream vortex. In the Fluent code, only the k- $\varepsilon$ turbulence model is available. 
Table 4.2 below lists the various parameters which can be varied or optimized for a given class of flow problems. For this study, the following values and settings were selected after much research into what are the most effective tools for these types of problems. The residual was evaluated at several levels, and decreasing the value by an order of magnitude appeared to consume more computer time without changing the results so a value of 0.001 was universally chosen.

Table 4.3 lists the boundary conditions used in this study. The free stream velocity was used for the inlet boundary condition. The velocity was entered as a vector assigned at the respective angle of attack. The same free stream velocity boundary condition was used for the top and bottom of the C-type grid. A pressure outlet boundary condition was assigned to the outlet. Standard sea level densities and viscosities were used.

Table 4.2: Solver parameters

\begin{tabular}{|l|l|}
\hline Viscosity & Laminar \\
\hline Spatial differencing & Second order upwind \\
\hline Temporal differencing & Backward Differencing \\
\hline Residuals & 0.001 \\
\hline Solver & Second order implicit pressure based \\
\hline Pressure velocity coupling & SIMPLE \\
\hline Gradient & Green-gauss cell based \\
\hline Reference area (3D model) & $0.05 \mathrm{~m}^{2}$ \\
\hline
\end{tabular}


Table 4.3: Boundary Conditions

\begin{tabular}{|l|l|}
\hline Frontal Inlet & Free stream velocity \\
\hline Top and Bottom & Free stream velocity \\
\hline Outlet & Pressure outlet \\
\hline
\end{tabular}




\section{Chapter 5}

\section{CFD Analysis of Leading Edge Flow Separation and Dynamic Roughness}

A computational fluid dynamics numerical analysis utilizing Fluent ${ }^{\circledR}$ was used to study both two and three-dimensional cases. Results of both tests predicted that a classic separation bubble does form on the upper surface downstream of the leading edge at moderate angles of attack at a Reynolds number of 100,000 and 150,000 on the NACA 0012 airfoil. These results were consistent with previous experimental data when compared at 9.5 degrees angle of attack [Rinoi and Takemura, 2004]. Laminar separation

tends to take place at about the $1.8 \%$ chord location at this angle of attack. Turbulent reattachment occurs downstream for both long and short bubbles. Both the numerical and experimental data indicate that the flow within the bubble is oscillatory and highly unsteady in nature. The two-dimensional results were used to validate the computational fluid dynamics predictions of the separation bubble on the baseline airfoil. The twodimensional results also compared well with the previous work of Huebsch [2006]. Since dynamic roughness is primarily a three-dimensional application, the focus of this research will be on the three-dimensional results.

\subsection{Basic Dynamic Roughness Model}

The basic CFD analysis was carried out in the following manner. 1) The NACA 0012 airfoil with 4.8 million cell volumes was run for several thousand time step iterations until the separation and reattachment point became established. Several different time 
steps were evaluated and it was discovered that a time step of 0.0002 yielded converging results. Applying too large of a time step lead to difficulties in the grid remeshing, smoothing and layering algorithms. Applying too small of a time step resulted in excessive computational time. This time step equates to a Courant number of about 3.0 based on the smallest cell characteristic length and a free stream velocity of 1.5 meters per second. This data, coined "baseline start case data", was saved as a start file for use in studying various dynamic roughness cases. 2) The baseline start case data was next interpolated and read into an appropriate mesh model for analyzing a specific dynamic roughness case.

Prior to analyzing dynamic roughness cases, an initial hump height and geometry as well as hump frequency needed to be selected as a starting point. Previous research by Huebsch [2006] utilizing a two dimensional Navier-Stokes solver applied to clean, static and dynamic roughness cases indicted that using a frequency of 60 Hertz and a hump amplitude equal to $50-80 \%$ of the incoming boundary layer height would possibly provide effective leading edge flow control. The model used in the reference research study was a parabola shaped leading edge region which approximated the geometry in the leading edge region of the NACA 0012 airfoil [Huebsch, 2006]. The first dynamic roughness hump was located at the $0.8 \%$ chord location, although the code had the capability to place a hump at the $0.6 \%$ chord location. This would place the first hump just upstream of the laminar separation point, which was previously found to be a necessary condition for effective flow control. At this location, the height of the incoming boundary layer flow is approximately $1.4 \mathrm{~mm}$ or $0.14 \%$ chord based on the data obtained 
from the baseline start case data. The roughness field itself consisted of twelve humps or ridges placed consecutively in the chordwise direction extending from $0.8 \%$ chord to $3.2 \%$ chord. The initial amplitude of the roughness was $0.8 \mathrm{~mm}$ or $57 \%$ of the height on the incoming boundary layer. The free stream velocity was 1.5 meters per seconds which corresponds to a Reynolds number of 100,000 . The initial frequency was chosen to be 60 $\mathrm{Hz}$.

It has long been understood that surface pressure, and the corresponding pressure gradient is one of the dominant factors in determining the behavior of the separation bubble [Sawada et al., 1976]. Therefore, the effects that dynamic roughness has on the pressure distributions in the leading edge region will be examined first. For the clean airfoil the basic pressure distribution is quite predictable and corresponds well with experimental data. The NACA 0012 airfoil, being an airfoil that is not designed for extensive laminar flow, has a pressure distribution curve that quickly peaks close to the leading edge where maximum suction pressure and maximum velocity are reached. Following the suction peak, the pressure begins to recover to free stream conditions, but due to the presence of the strong adverse pressure gradient, the flow can no longer accommodate the curvature of the airfoil and so it separates just downstream of the suction peak. This results in a smooth and rather gradual pressure recovery. This can be observed in figure 5.1, which focuses on the upper surface pressure coefficient very close to the leading edge. The pressure distribution for the dynamic roughness in this figure represents a temporal snapshot of the humps when they are at maximum amplitude 
during the expansion-contraction cycle. This case was run at 12 degrees angle of attack and a Reynolds number of 100,000 .

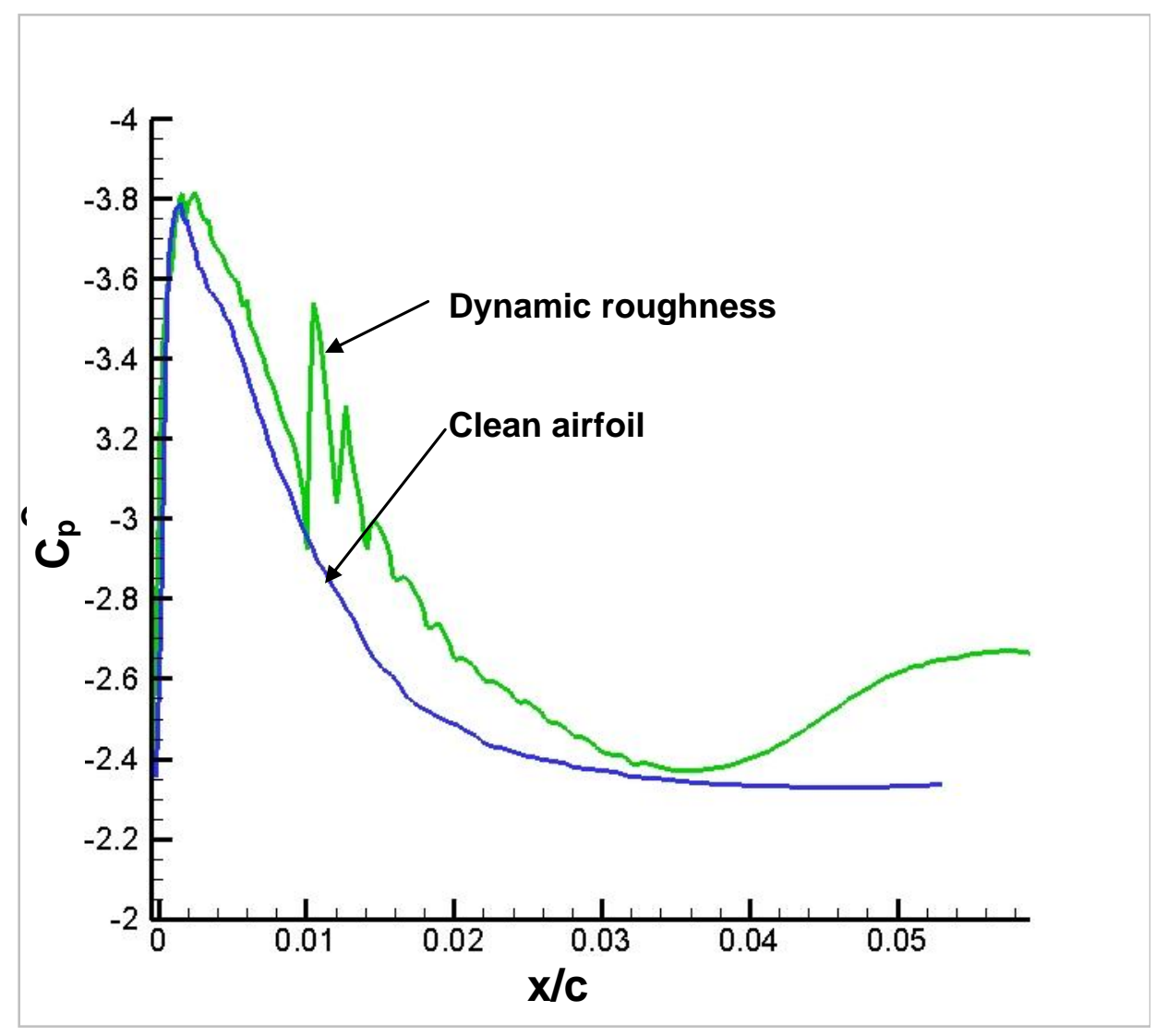

Figure 5.1: Clean airfoil and dynamic roughness airfoil leading edge pressure distributions

When the dynamic roughness is actuated, it creates artificially induced pressure gradients within the boundary layer which tend to accelerate and decelerate the flow locally. A slight but sudden recovery of suction pressure is observed at the first hump. Downstream of the first hump, the suction pressure appears to increase throughout the roughness region $(0.8 \%$ to $3.2 \%$ chord $)$. It is also interesting to note that the slope of the pressure curve (pressure gradient) appears to become less adverse when dynamic roughness is 
applied. A final observation is that the dynamic roughness delays the appearance of the sharp adverse pressure gradient until further downstream. The combination of these three effects (greater negative pressure, less adverse pressure gradient and shifting of pressure recovery downstream) contribute to the beneficial effects of dynamic roughness in eliminating the separation bubble.

It is thought that the dynamic roughness is an effect felt within the laminar boundary layer. Flow control, at least at this stage, is not believed to be caused by the boundary transitioning to a fully turbulent boundary layer. It is for this reason that the code was run without any turbulence modeling. Therefore, all calculations were based on laminar flow physics (section 4.3). In reality, it is likely that at some point downstream of the roughness field, once the laminar boundary layer encounters the dynamic humps and "receives" effective flow control the state of the boundary layer may transition to a turbulent flow.

Examining the pressure distributions further downstream one finds that the beneficial effect of the dynamic roughness tends to carry itself downstream as well. This can be observed in figure 5.2. This pressure distribution is an integrated average in time and spanwise space for the three-dimensional hump model. The pressure coefficients were averaged over a period of 3 cycles which equates to a flow time of 0.050 seconds. Even though these are average values, the fluctuation of pressure is still evident, particularly over the first couple of humps. 
At approximately the $7 \%$ chord location, an increase in suction pressure can be seen for the dynamic roughness case. This was due to the formation of an unsteady vortex downstream of the dynamic roughness. This appears to be an artifact of using a laminar solver (this was found to be common in this and other studies when applying a laminar solver). In order to verify this, several cases were run where the flow, after passing through the dynamic roughness field, entered a zone where the k- $\varepsilon$ turbulence model was introduced. This was at the $3.4 \%$ chord location. Although the exact location of transition is not a known priori, it is believed that at some point downstream of the dynamic roughness region, the boundary layer would undergo a natural transition to turbulence. This would lead to attached flow downstream, as was observed in the experimental studies. 


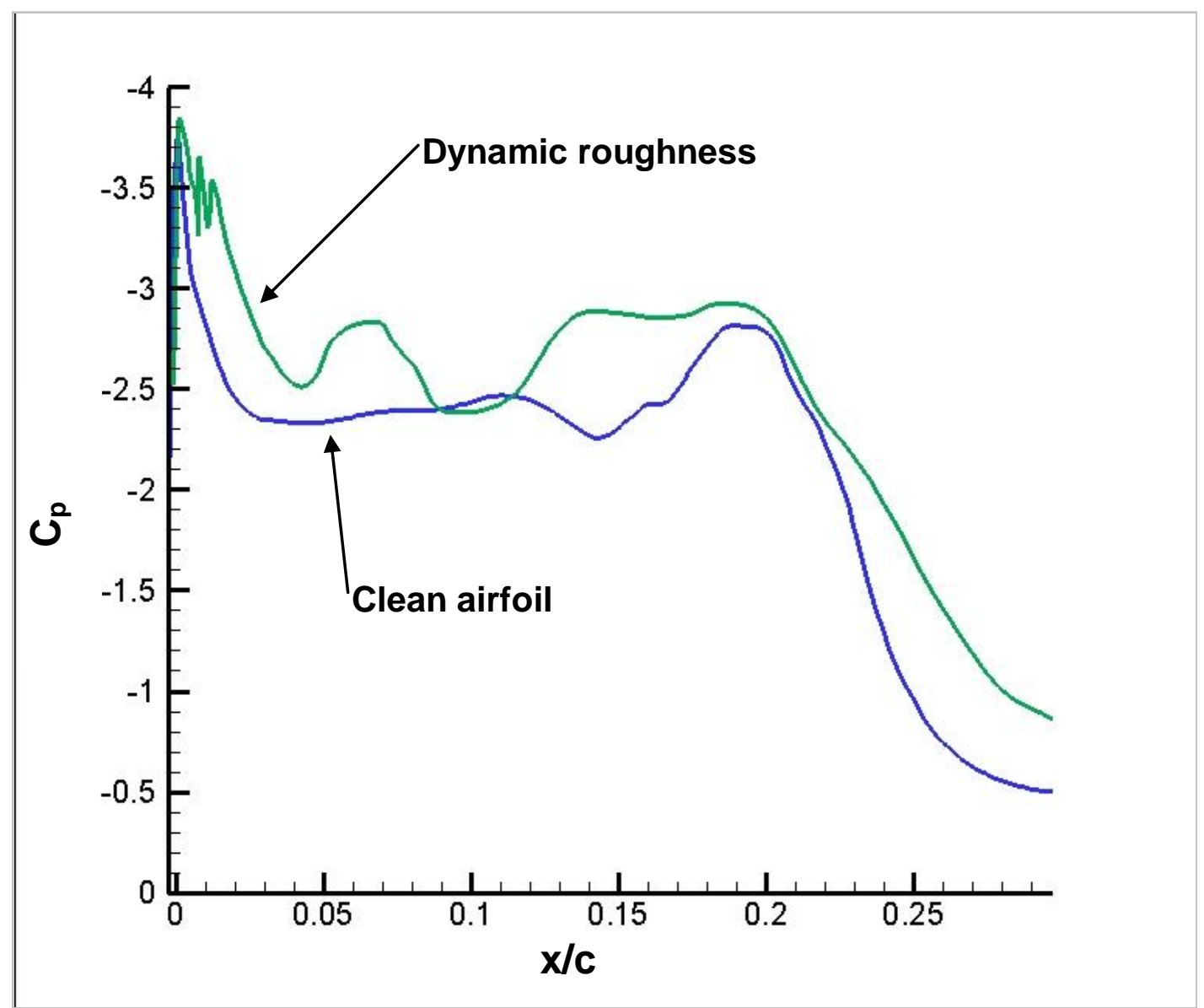

Figure 5.2: Clean airfoil and dynamic roughness airfoil pressure distributions (time and spaced averaged)

It is also of interest to examine the pressure history over the hump region for one full cycle of hump motion. Figure 5.3 is a plot of the pressure coefficient at four different hump positions as it moves through one cycle. The four geometric positions are the following: 1) humps halfway up 2) humps full amplitude 3) humps halfway down and 4) humps flush with surface. The plot depicts data back to the $5 \%$ chord location so that the pressure effects just downstream of the roughness field can be observed. The intent is to show how the pressure varied as a function of hump position. It can be observed that although the overall magnitude of the pressure coefficient changes, the slope of the 
pressure coefficient curve does not vary significantly. This directly correlates with the conceptual studies of Rothmayer and Huebsch [2010]. It is unclear how sensitive the boundary layer may be to very small changes in the pressure gradient. As previously discussed, it appears that the effect of the dynamic roughness is to alter the flow physics in such a fashion that the boundary layer separation may be delayed and/or eliminated entirely. This combined with the resulting pressure distributions tends to produce favorable global effects on the lift and drag coefficients.

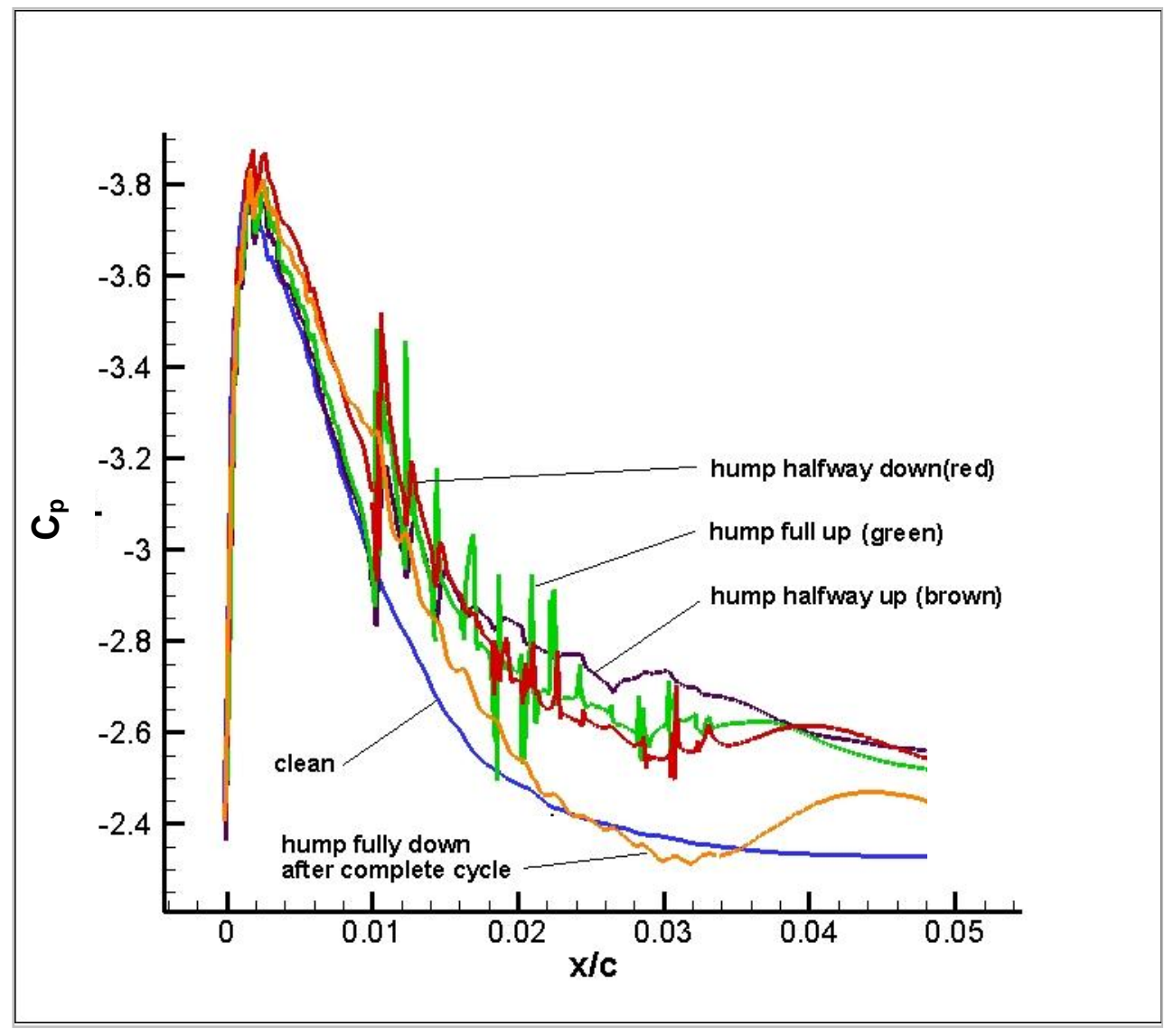

Figure 5.3: Dynamic roughness pressure distributions throughout hump cycle

The pressure distribution over an individual hump or series of humps is also of particular interest. Figure 5.4 shows the pressure distribution over the first two humps beginning at 
time $t_{0}$, the point at which the humps are flush with the airfoil surface. These humps are located at the $0.8 \%$ to $1.2 \%$ chordwise position. This figure shows the pressure distributions over the humps as they transition through one full cycle of motion. It was decided to illustrate the case of two humps since the pressure changes are much more pronounced over the first hump than the second hump. As one continues downstream, the peaks and valleys of the pressure fluctuations tend to dampen out. This was due to the fact that the downstream humps lie in the wake of the first hump. It can be observed that when the humps are flush we are observing the basic clean airfoil case data pressure distribution. The numbers labeled on each curve in figure 5.4 denote the respective hump position as denoted in figure 5.5. At position number 9 the humps are almost flush with the surface. It can be observed that the pressure has not returned to the level that existed prior to the initial hump expansion. It appears that when the humps retract they leave behind a residual pressure change. Figure 5.5 is a plot of the actual time history of the hump motion. It was based on a sine wave function. The hump rises and falls quickly. The actual physical time of the hump expansion and contraction is based on the flow time and hump frequency. Figure 5.6 is a cross sectional profile view of a given hump three at selected time steps. Since the humps are axi-symmetric, this cross section is taken at the chordwise centerline of the respective hump. For the cases of the ridges, it can be considered a cut through any spanwise section of the ridge in the mid-span region. 


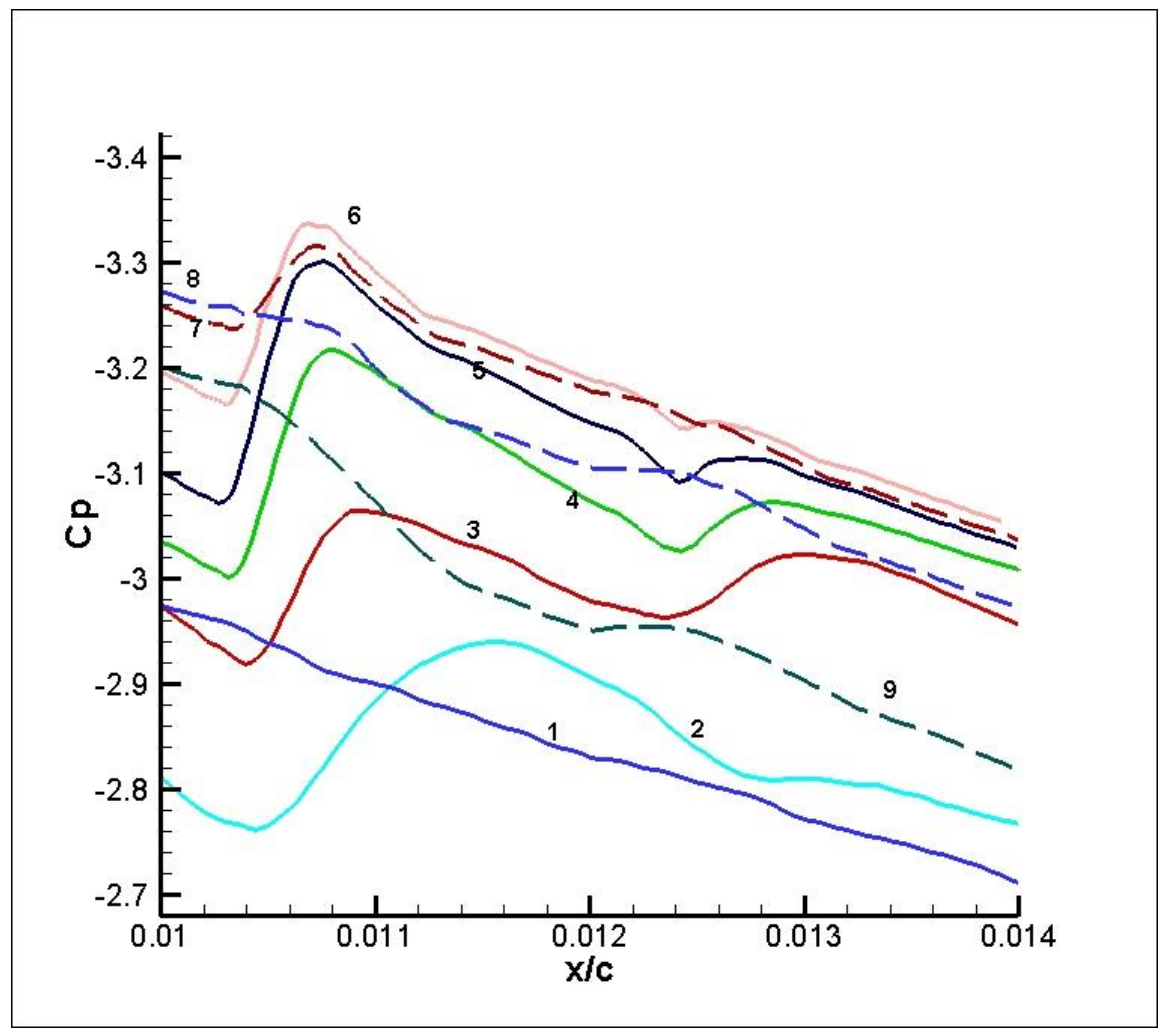

Figure 5.4: Pressure coefficient over first two humps throughout one cycle

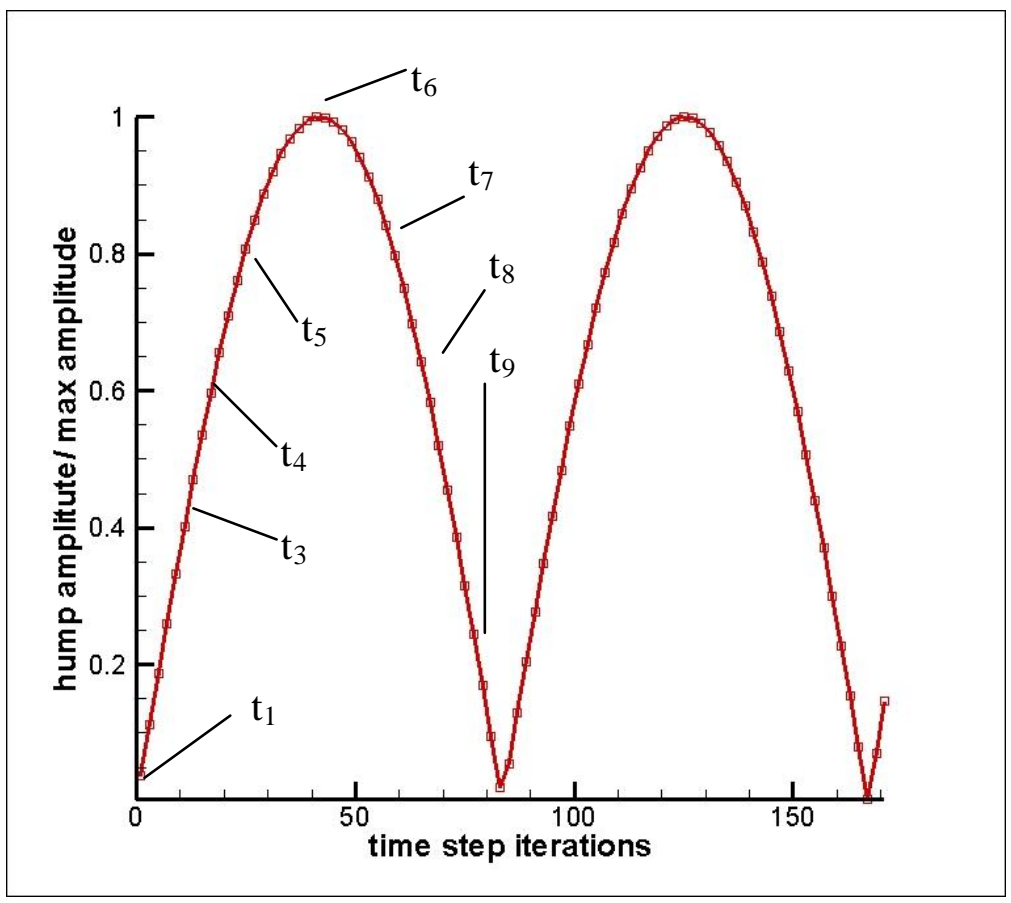

Figure 5.5: Time history of hump motion 


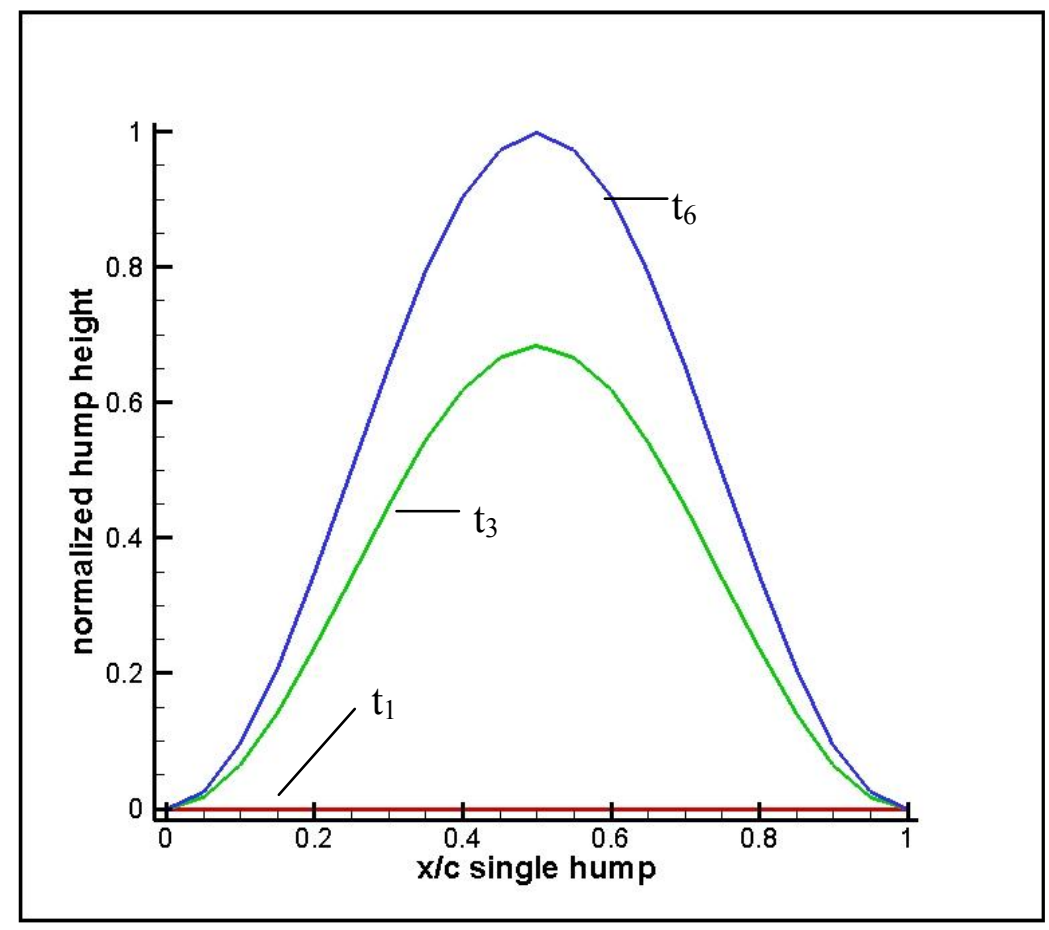

Figure 5.6 Displacement history of hump motion

For a partial validation of the numerical calculations, a computed pressure distribution was compared to experimental values. Figure 5.7 is a plot of the computed pressure distribution for the above mentioned case at an angle of attack of 9.5 degrees and a Reynolds number of 100,000 . The pressure distribution is time averaged over several hundred time steps. The experimental data is digitized data taken from Rinoi and Takemura [2004], where the NACA 0012 airfoil was tested at a slightly higher Reynolds number of 130,000 . Close agreement can be observed in the data, particularly in the separation and reattachment regions extending from near the leading edge to approximately the $20 \%$ chord location. 


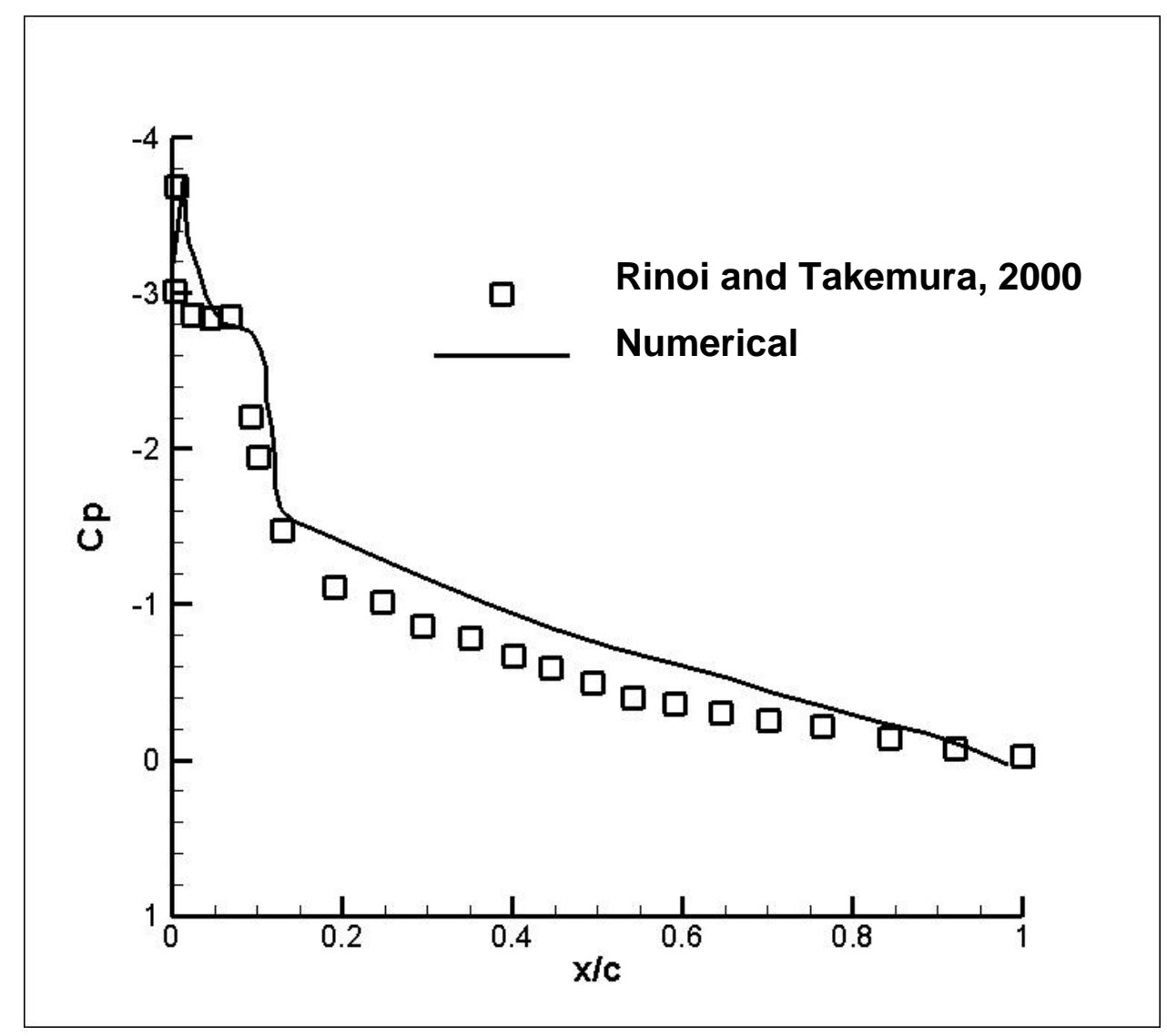

Figure 5.7: Experimental and numerical pressure coefficient data for 9.5 degrees angle of attack and Reynolds number 100,000

The next area of interest was to examine the boundary layer velocity profile in the leading edge region. It was decided to sample three locations in the region. The first rake was located upstream on the separation bubble and dynamic roughness field. The second rake was placed midstream in the dynamic roughness field. The third rake was placed just downstream of the last hump location. In percent chord location, this puts the rake locations at $0.6 \%, 1.8 \%$ and $3.2 \%$ chord positions, respectively.

Figure 5.8 shows the respective velocity profiles at the $0.6 \%$ chord location. For each location two cases are examined; the first is the case of the clean baseline airfoil and the 
second is the case of the dynamic roughness model. The parameters evaluated for the dynamic roughness cases are 12 degrees angle of attack, 100,000 Reynolds number, hump frequency $60 \mathrm{~Hz}$, hump amplitude equal to $57 \%$ of incoming boundary layer height, and twelve humps placed consecutively from $0.8 \%$ to $3.2 \%$ chord. This results in 300 individual humps. For each case the velocities are shown for $u$, the velocity parallel to the surface. It can be observed in figure 5.8 that the velocity profiles look very similar to classic laminar boundary layer velocity profiles, as one would expect in this region. Since the rake is sampling the velocity two hump lengths upstream of its location, one can observe that the velocity of the flow near the wall for the dynamic roughness case was decelerating and the velocity away from the wall was accelerating. This data was for the hump fully extended $0.8 \mathrm{~mm}(0.08 \%$ chord$)$. 


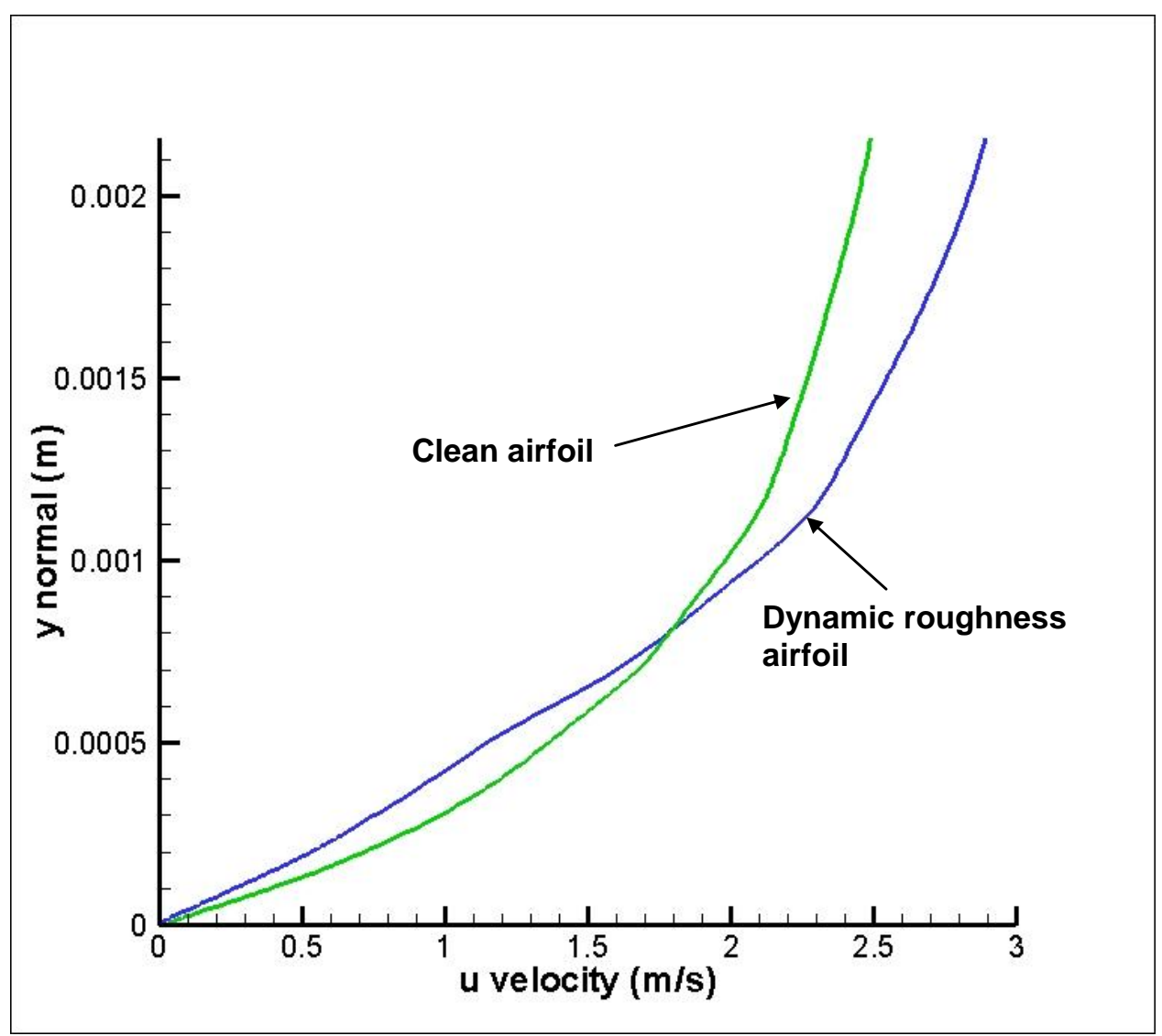

Figure 5.8: Boundary layer velocity profile at $0.6 \%$ chord location

In the case of the second rake location (figure 5.9), a change can be observed in the velocity profile. In the case of the clean airfoil, the flow near the surface is nearly stagnant. The clean velocity profile takes on a characteristically unstable profile. This is the forward region of the separation bubble and this is consistent with experimental findings [Sawada et al., 1976]. For the dynamic roughness case an inflection in the velocity profile can be observed when the boundary layer thickness is approximately the amplitude height of the hump. This was due to the region of reverse flow near the wall. This represents the region in between the peaks of the humps and indicates an area where a small vortex existed. As one moves away from the wall, beyond the height of the hump, the boundary layer takes on a fuller and more stable profile. 


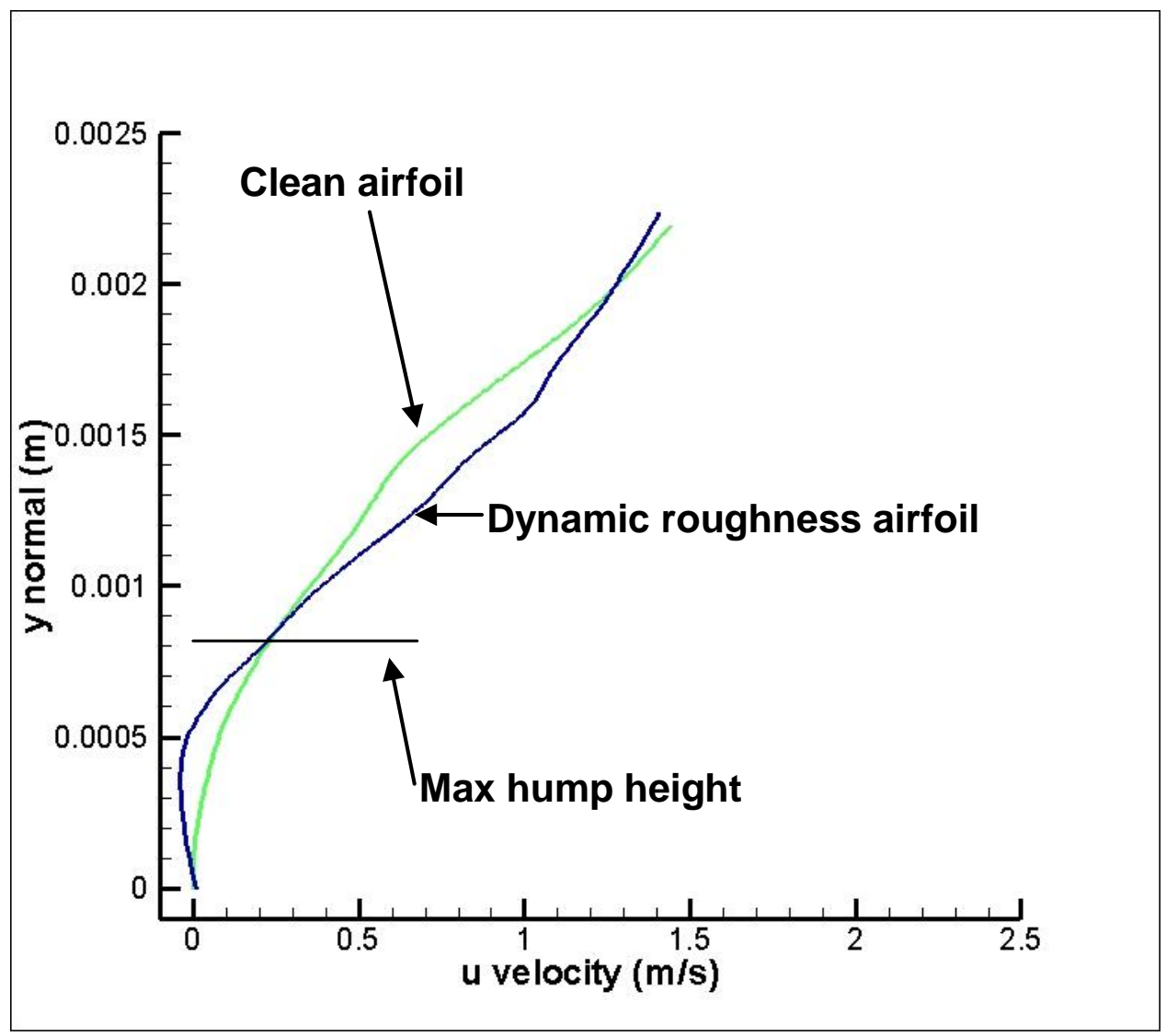

Figure 5.9: Boundary layer velocity profile at $\mathbf{1 . 8 \%}$ chord location

The 3.4\% rake location was just downstream of the last hump. At this location the flow was separated in the clean airfoil case and the velocity profile is unstable (figure 5.10). In the dynamic roughness case, the flow has passed over the last hump. Just aft of the last hump is a small vortex (region of reverse flow) which does not convect downstream. After passing this vortex, the boundary layer attaches to the surface. Above the roughness height, the profile is also stable and non-inflectional. 


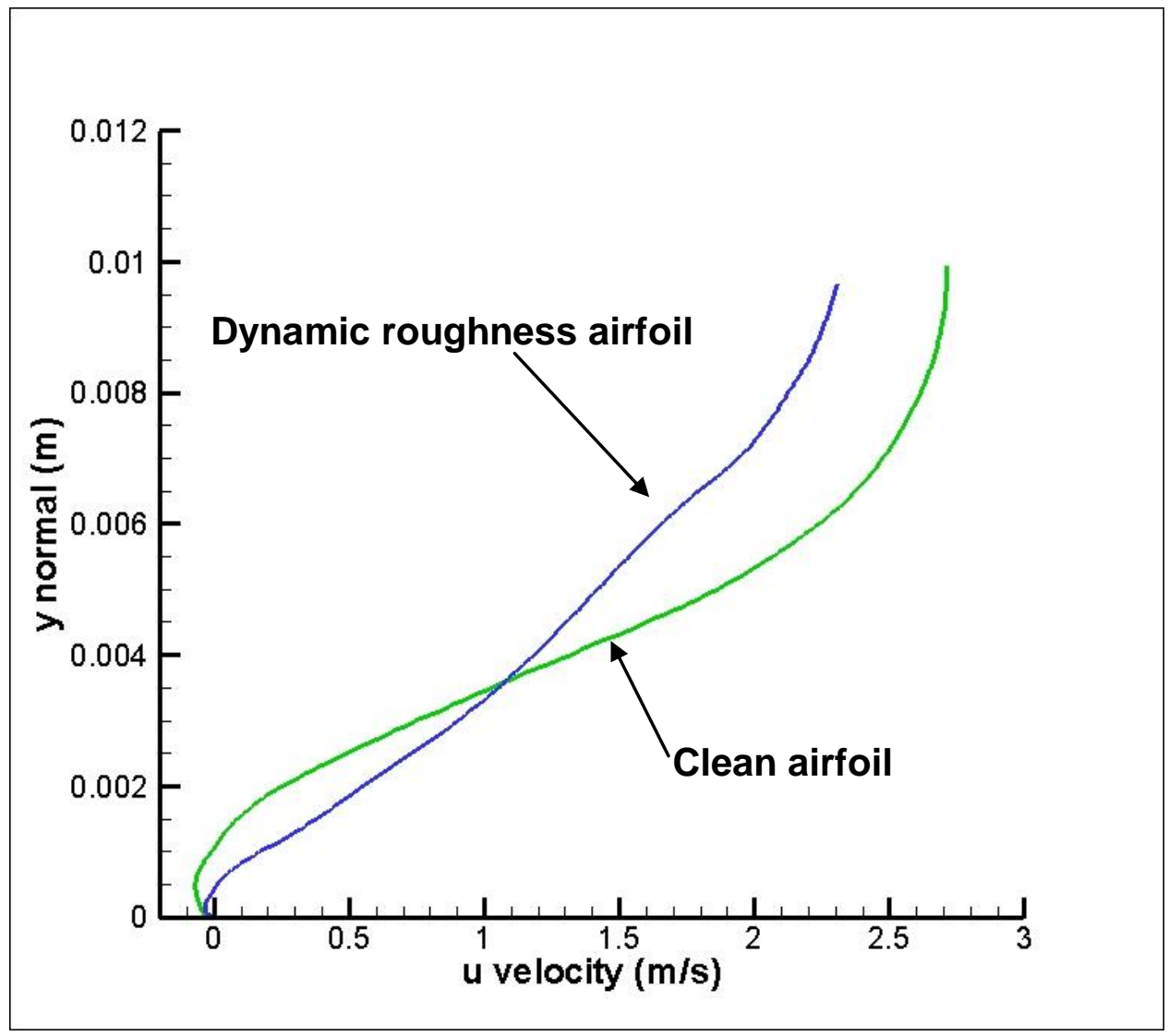

Figure 5.10: Boundary layer velocity profile at $3.4 \%$ chord location

Table 5.1 gives a listing of the parameters applied in this particular calculation. Many of the parameters and the selected values have been previously discussed. 
Table 5.1: Dynamic Roughness Parameters

\begin{tabular}{|l|l|l|}
\hline & Clean Airfoil & Dynamic Roughness \\
\hline Velocity & 1.5 meters/second & 1.5 meters/second \\
\hline Reynolds number & 100,000 & 100,000 \\
\hline Angle of attack & 12 degrees & 12 degrees \\
\hline Chord and span & 1.0 meter x 0.050 meter & 1.0 meter x 0.050 meter \\
\hline Airfoil & NACA 0012 & NACA 0012 \\
\hline Hump geometry & none & Axi-symmetric \\
\hline Hump location & none & $0.8 \%-3.2 \%$ chord \\
\hline Hump frequency & none & $60 \mathrm{~Hz}$ \\
\hline Hump amplitude & none & $0.08 \%$ chord $(0.8 \mathrm{~mm})$ \\
\hline
\end{tabular}

Figure 5.11 shows a display of two wing sections, one with dynamic roughness and the second a clean surface. The favorable effects of the dynamic roughness can be observed in the pathlines as well as the surface pressure contours. For the clean case, the flow clearly separates from the surface. The flow remains separated creating a large separation bubble. In the case of dynamic roughness, the flow remains attached throughout the roughness field and remains attached further downstream. This illustration depicts the flow field after the humps have moved through 3 cycles. It is important to note that once the dynamic roughness is actuated, there is a transient period in which the boundary layer changes from a separated state to an attached state. The length of this transient period varies, depending on the frequency. For the $60 \mathrm{~Hz}$ case, the transient behavior lasts about 2 cycles. A similar transient period was also seen in the flow visualization work. 
Eventually the flow separates further downstream when the adverse pressure gradient resulting from the pressure recovery creates instabilities that the boundary layer cannot overcome. Based on experimental results presented below, it is believed that this eventual downstream separation is an artifact of forced laminar flow and is not physical.

A second observation is the three-dimensional surface pressure. When the flow becomes attached to the surface in the field of the dynamic roughness, the improvement in the suction pressure can be observed. This directly leads to more lift and less drag in the form of favorable "leading edge suction".

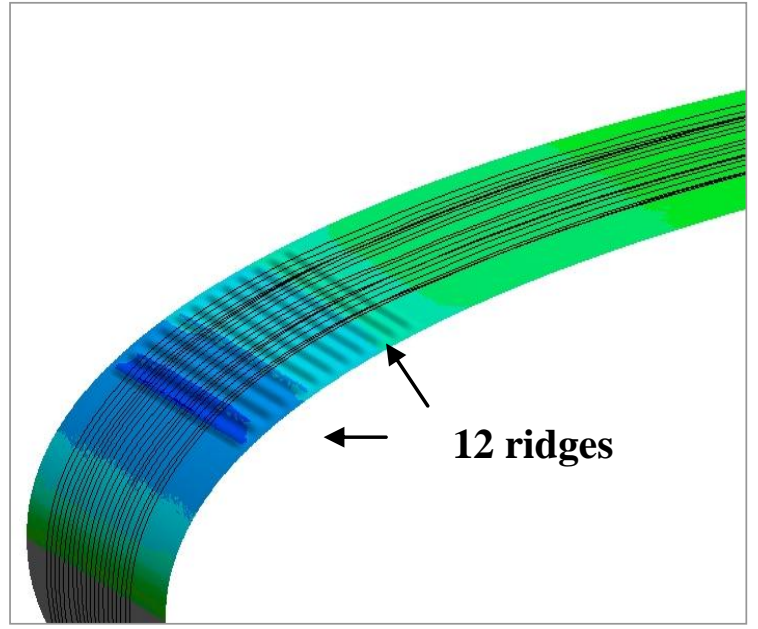

a) Dynamic roughness airfoil

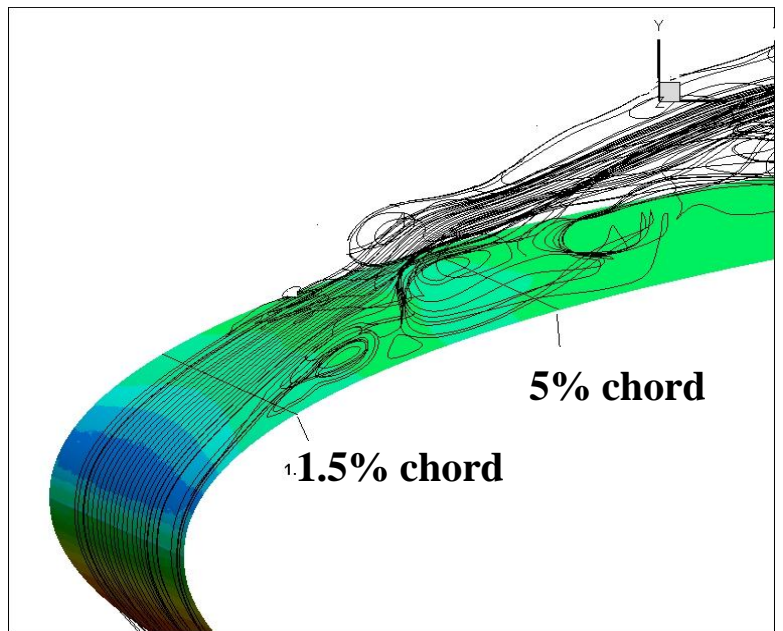

b) Clean airfoil

Figure 5.11: Surface pressure contour and pathline plots of clean airfoil and airfoil with dynamic roughness ridges (12 degree angle of attack and 100,000 Reynolds number)

It should be noted that the dynamic roughness wing in this case used time-dependent ridges instead of three-dimensional humps. Both the ridges and the humps provide flow control and eliminate the leading edge separation bubble. The results of this study 
indicated that there was little difference when comparing the effects of ridges and humps on flow control.

Numerical calculations were carried out to examine the cases of a clean airfoil, an airfoil with static roughness and an airfoil with dynamic roughness. One of the specific objectives of this study, based on the previous work of Huebsch [2006], was to examine the case of static roughness and compare to clean and dynamic roughness surfaces. When one considers the application of critical roughness Reynolds number height to estimating roughness heights for boundary layer transition [Braslow, 1966] it was determined that an $\mathrm{Re}_{\mathrm{k}}$ of 600 is required to cause a premature transition of the boundary layer on an airfoil. This equates to a roughness height of $4.3 \mathrm{~mm}$ or $0.4 \%$ chord for the first roughness element. If the dynamic roughness was held "statically " at a maximum amplitude of 0.8 $\mathrm{mm}$, a roughness Reynolds number of 120 would be reached. This would be far below the 600 value, which would be required if the objective was to use static roughness as a boundary layer trip. At this amplitude, the roughness Reynolds number would only be about $20 \%$ of the roughness height required to cause transition based on Braslow's findings. For this reason, it was desirable to study the case of static roughness to verify that it would not change the laminar separation point.

In all cases the clean start case data was first interpolated and read into the respective case. Figure 5.12 shows a display of the three-dimensional model after 40 time step iterations or a flow time of 0.08 seconds. The intent of this figure is to show that this type of flow control is indeed a transient process. After the hump motion has began, there is a 
transient time period which the flow transitions from separated to attached. After three cycles of hump motion at a frequency of $60 \mathrm{~Hz}$, the flow becomes attached and remains attached. The contours represent the pressure coefficient levels. At each time step, the solution was iterated until convergence, that is, until the convergence parameter residuals converged to values less than 0.001 .

In the case of the clean airfoil, there was no change in the separation point (figure 5.12(a)). Although the flow around the bubble is quite unsteady, the overall characteristics of the bubble do not change. In the case of the static roughness (figure 5.12(b)) the separation point again stays at the same chord location. The large vortex which is formed tends to convect rearward. There is a short area of reattachment behind where the first vortex reattaches and the second vortex begins to form. In the case of the dynamic roughness (fig. 5.129c)), the humps have started to expand and have almost reached their maximum amplitude at the beginning of their first cycle. The separation point has began to shift rearward. This is the first indication of an effective means of flow control. The intent of these figures is to show that once the dynamic roughness is actuated, there is a transient stage (as previously discussed) which occurs before the full flow control takes effect. From the pressure distribution calculations it can be observed that a favorable pressure gradient has been artificially produced near the region of the first hump. It can also be observed that as the flow passes through the hump region, the pressure effects become less pronounced. 


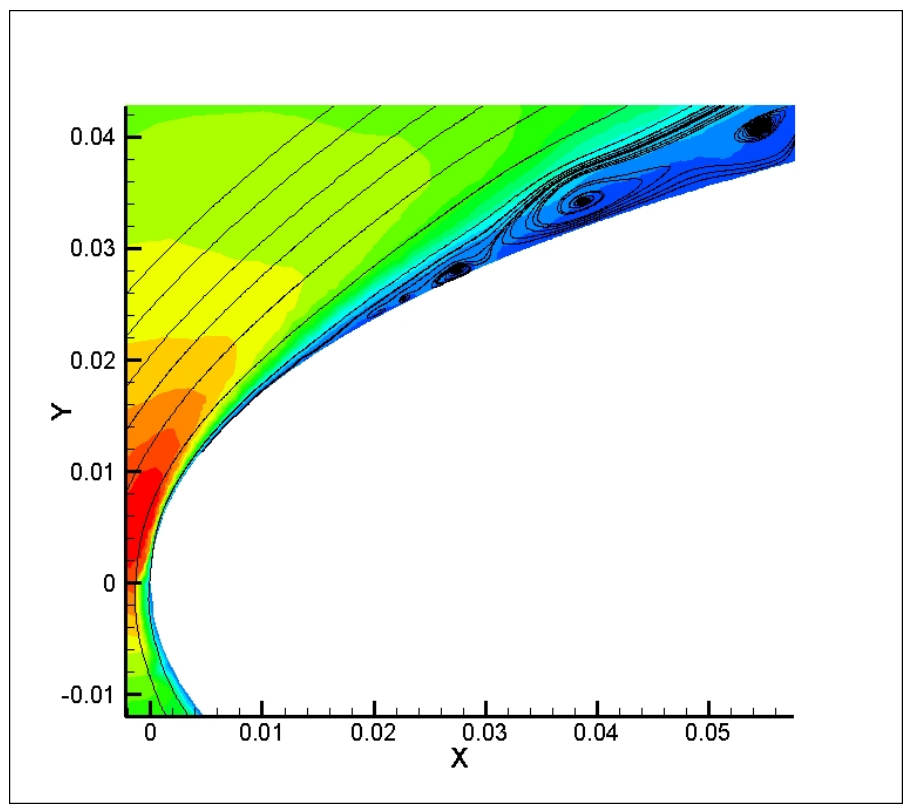

a) Clean airfoil

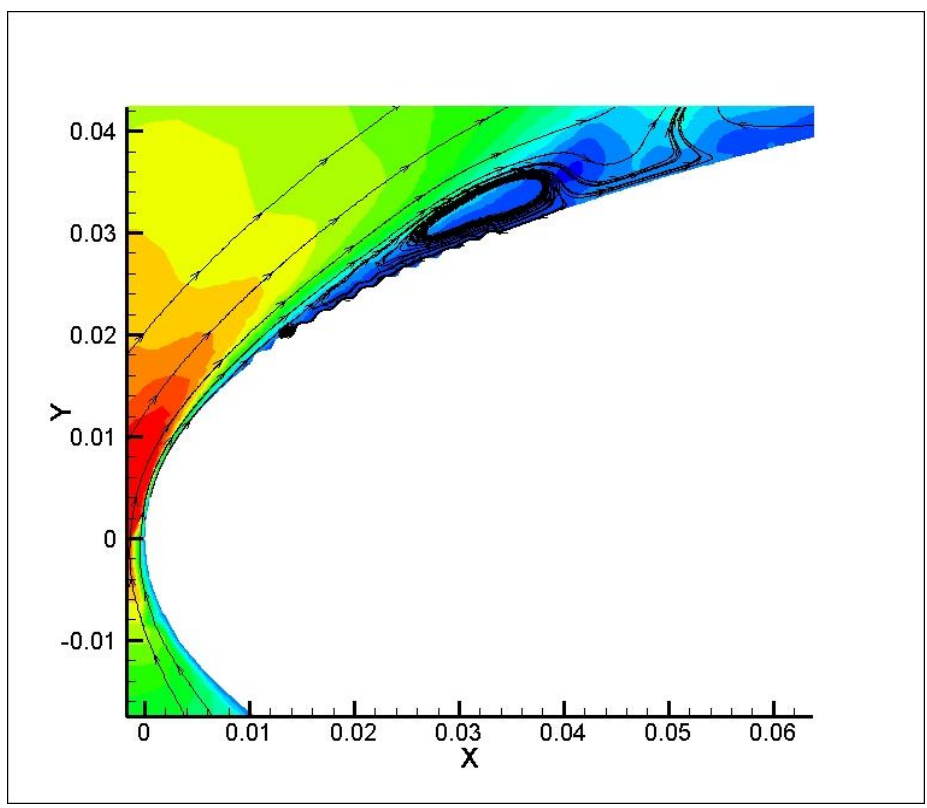

b) Static roughness airfoil 


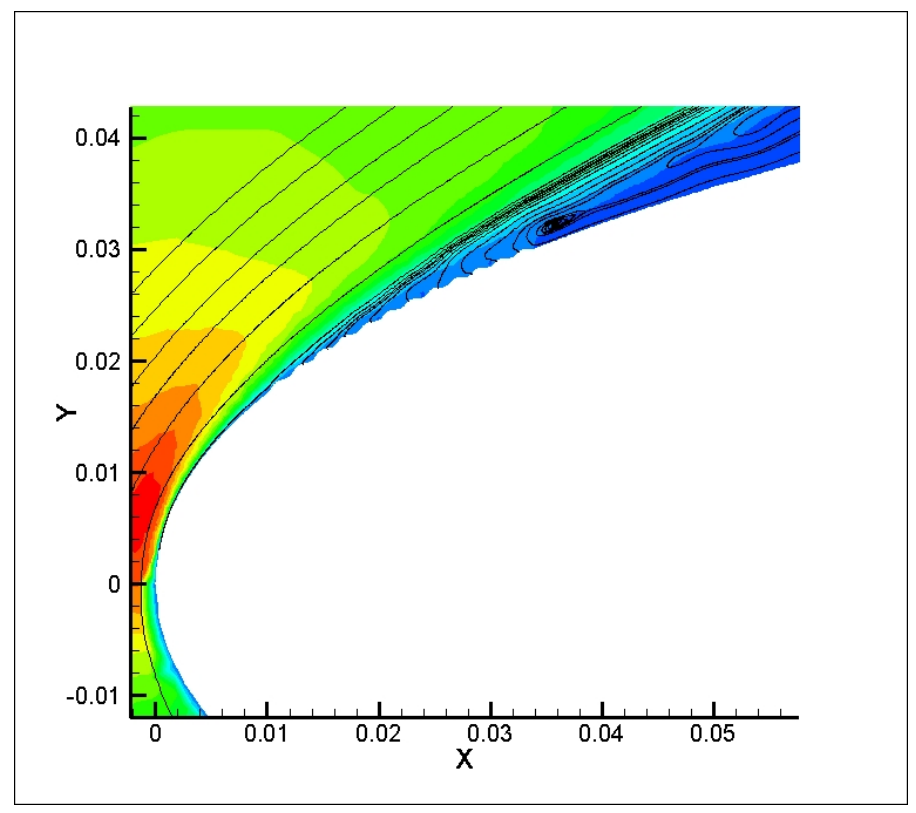

c) Dynamic roughness airfoil

\section{Figure 5.12: Pressure contour and pathlines of a) clean, b) static, and c) dynamic} roughness airfoils at time $t_{1}$

At the next point in time, indicated as $t_{2}$, the humps have undergone $2-1 / 2$ cycles. In the clean airfoil case (figure 5.13(a)), the separation point has remained at the same location. The general characteristics of the bubble have not changed although the flow is highly unsteady. In the static roughness case, the large vortex has convected downstream (figure 5.13(b)). It is interesting to note that in the static case it can be observed that as the existing vortex convects downstream, a new vortex begins to form near the separation point. If we continue to march forward in time we notice that as one vortex convects downstream, a new vortex is spawned near the separation point. This pattern repeats itself over and over while the overall characteristics of the separation bubble do not change. 
In the case of the dynamic roughness (figure 5.13(c)) the flow remains attached to the surface of the airfoil after passing through the roughness field. Throughout the roughness field, while the humps were expanding and contracting, the flow seemed to form a thin shear layer within the region. Basic flow physics dictates that once the flow passes clear of the dynamic roughness it is free to separate if conditions warrant a separation. These conditions would be consistent with what would cause the separation bubble to form in the first place, namely a laminar boundary layer and a sufficiently adverse pressure gradient. By observing figures 5.12(c) and 5.13(c) it can be observed that there is a transient type behavior that takes place between when the dynamic roughness is actuated and the flow control begins to take effect.

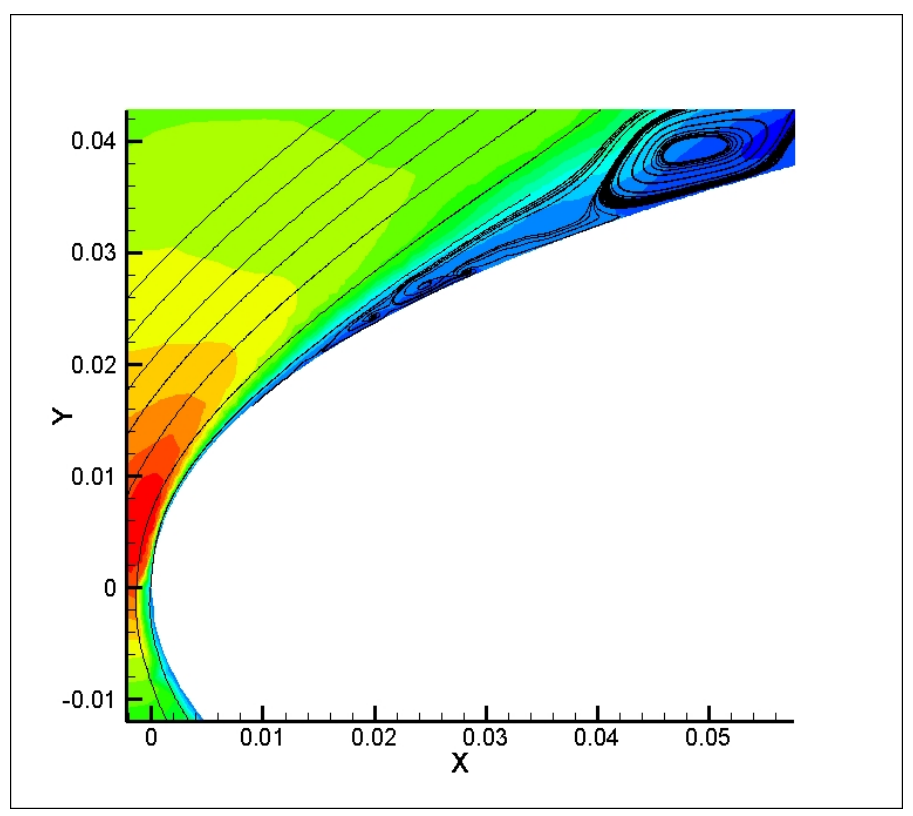

a) Clean airfoil 


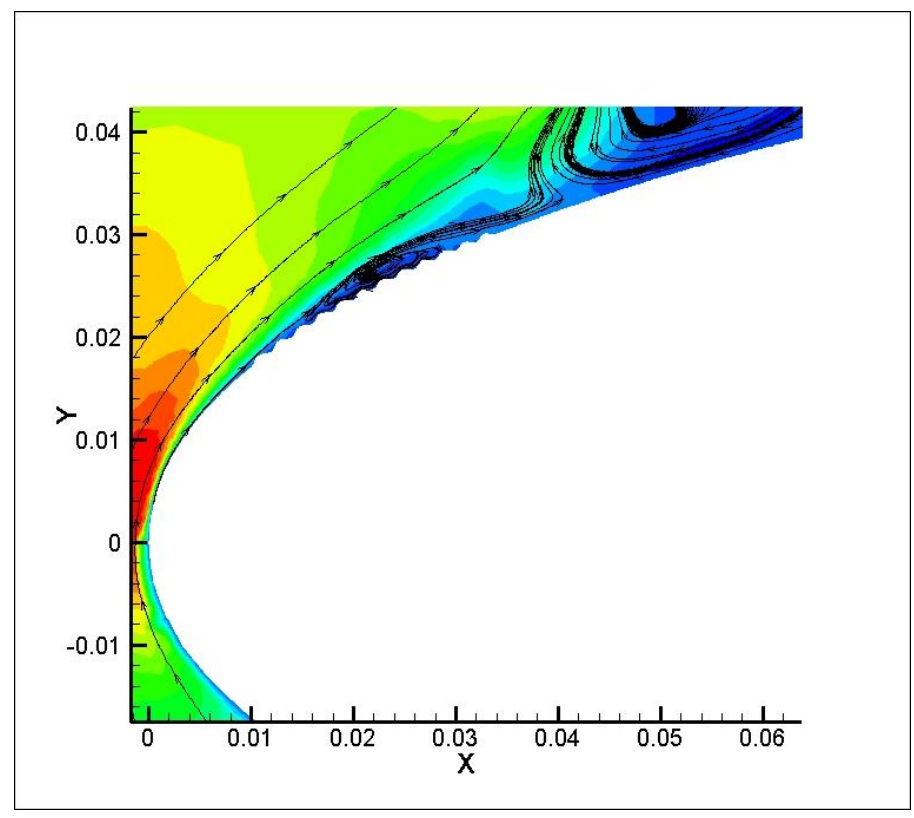

b) Static roughness airfoil

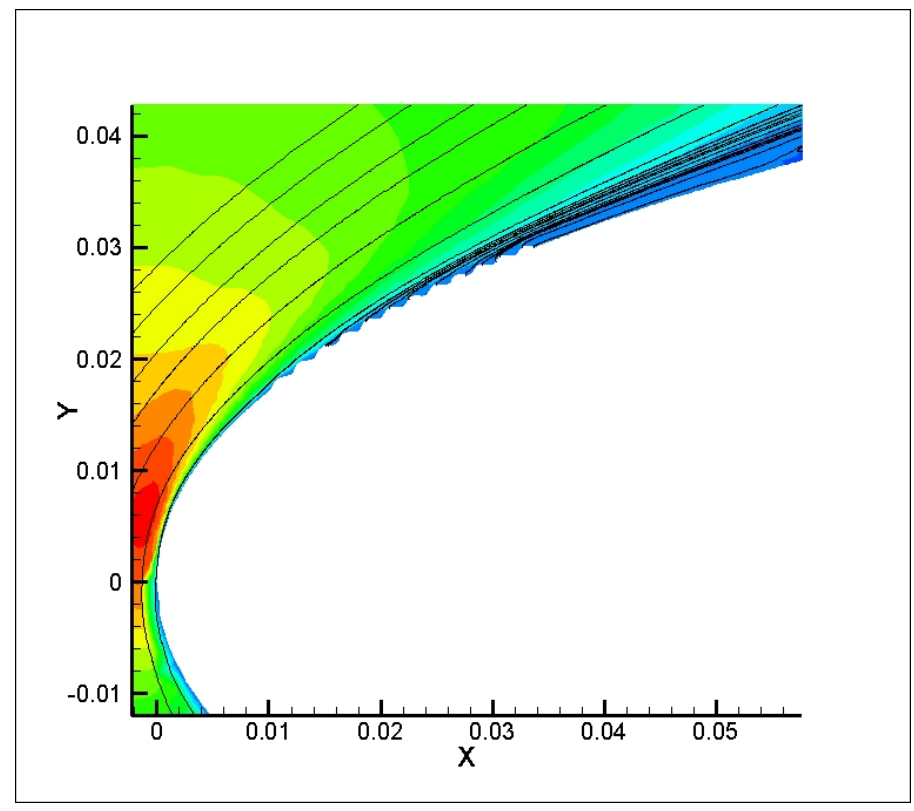

\section{c) Dynamic roughness airfoil}

Figure 5.13: Pressure contour and pathlines of a) clean, b) static, and c) dynamic roughness airfoils at time $t_{2}$

Once the humps have contracted to the flush position, as shown in figure 5.14, it can be observed that the flow not only remains attached, but the influence of the humps on the 
pressure field can be seen. The humps tend to leave behind a "footprint" of their presence. It is believed that the small-scale pressure gradient signatures left behind may contribute to the small-scale mixing that is occurring near the wall. This is in essence a thin shear layer located at the wall.

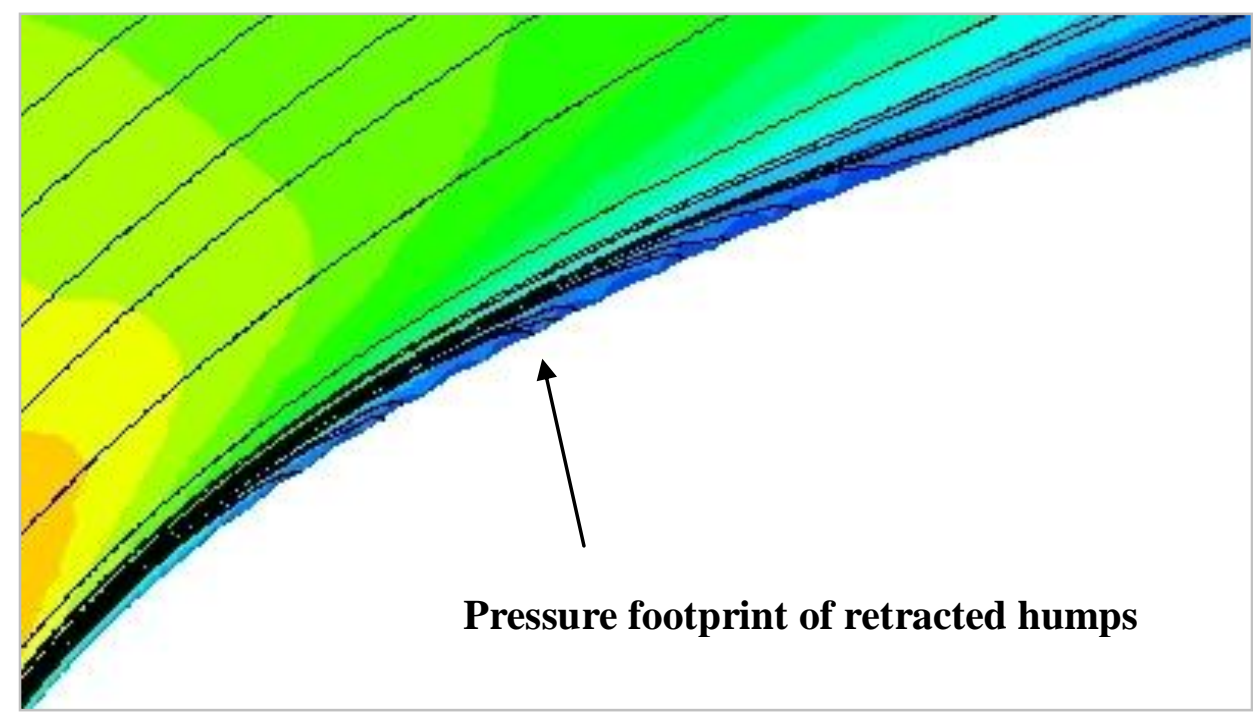

Figure 5.14: Pressure contour and pathlines of dynamic roughness airfoils at time $t_{3}$

Figure 5.15 is an illustration of the three-dimensional pathlines such that the flow patterns for the three cases of clean, static roughness and dynamic roughness can be observed. Figures 5.15(a) and 5.15(b) show the leading edge flow separation for the clean airfoil and the airfoil with static roughness. Figure 5.15(c) is the case of the dynamic roughness after $2-1 / 2$ cycles. The pathlines clearly show how the flow has changed from a classic separation bubble to an attached boundary layer type flow. In these three cases, the flow rake used to generate the pathlines was located at the same location at the leading edge. 

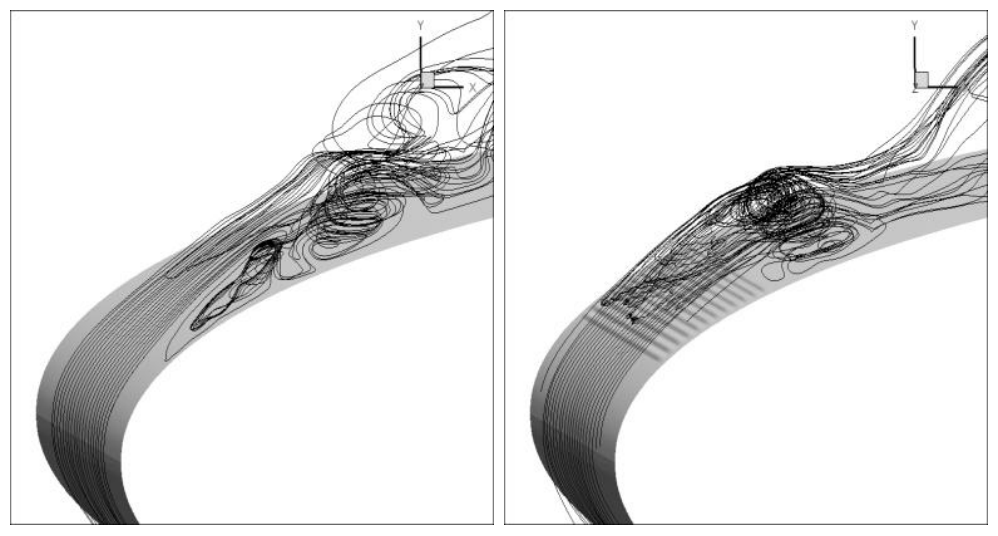

b) Static roughness airfoil

\section{a) Clean airfoil}

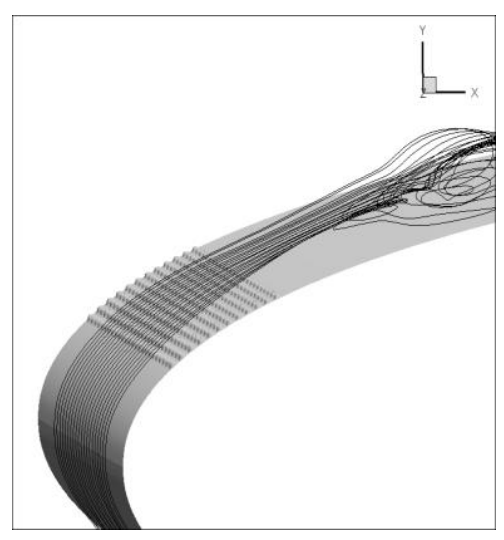

c) Dynamic roughness airfoil

\section{Figure 5.15: Three-dimensional pathline plots of clean, static and dynamic}

roughness cases

A fundamental part of understanding the benefits of flow control lies in understanding the changes effective flow control have on the local shear stress distribution. The drag of an airfoil primarily consists of two components, the tangential viscous shearing forces and the normal pressure forces. Immediately downstream of the stagnation point, the shear stress levels are normally quite high due to the early development of the boundary layer and the large values of the velocity gradient near the wall. As the boundary layer develops, the magnitude of the velocity gradient decreases and the shear stress levels decrease. When the boundary layer is turbulent, the velocity gradient is much higher than in the laminar case, and hence the turbulent skin friction drag is much higher.

Figure 5.16 is a plot of the surface shear stress levels in the leading edge region of the airfoil at 12 degrees angle of attack and a Reynolds number of 100,000. Included in this figure are wall shear stress results for a clean wing using laminar and turbulent flow simulations and a dynamic roughness wing using the laminar solver. Let us consider the flow over the bubble region. In this region the flow is relatively stagnant near the wall 
except where a vortex exists close to the surface. For this reason the shear stress levels are quite low. Considering the roughness region, there exits a shear layer over the humps where the flow is close to stagnant near the wall. In the valleys located between the humps the shear stress is low. At the peaks of the humps, where the flow is attached, a rise in the local shear stress levels can be observed. As this shear layer thickens further downstream, this effect becomes less and less pronounced so that the overall effect is a significant reduction in local shear stress versus the turbulent attached case, which is also shown in figure 5.16 for comparison purposes. For the clean wings, if turbulent flow is available, it is able to suppress the separation bubble, but of course has a higher wall shear stress than the laminar case. The dynamic roughness wing was also able to suppress the bubble, but has similar wall shear to the clean laminar case. This translates to a drag reduction.

In practice, artificial trips are often used to trip the leading edge flow to turbulent in order to avoid the overwhelming adverse affects of the bubble. Dynamic roughness may offer a way to capture the benefits of both scenarios, the favorable pressure and high lift characteristics of the turbulent boundary layer and the reduced viscous shear stress of the laminar boundary layer.

As a note of interest, Stratford [1959], after some study on airfoil boundary layers, examined the possibility of diffusing a turbulent boundary layer by prescribing a pressure distribution such that the shear stress at the wall would be zero. This became known as "imminent separation pressure recovery". He found that is was indeed possible to 
maintain a velocity profile such that the velocity gradient at the wall would remain zero. Hence the boundary layer remains on the verge of separation since a negative value would indicate indeed a flow reversal. This "Stratford distribution" was adopted by Liebeck [1970], and used to design an airfoil that would prescribe to this pressure distribution. Although this design showed some promise in theory, it unfortunately carried some very adverse characteristics at high angles of attack, making it undesirable for practical applications.

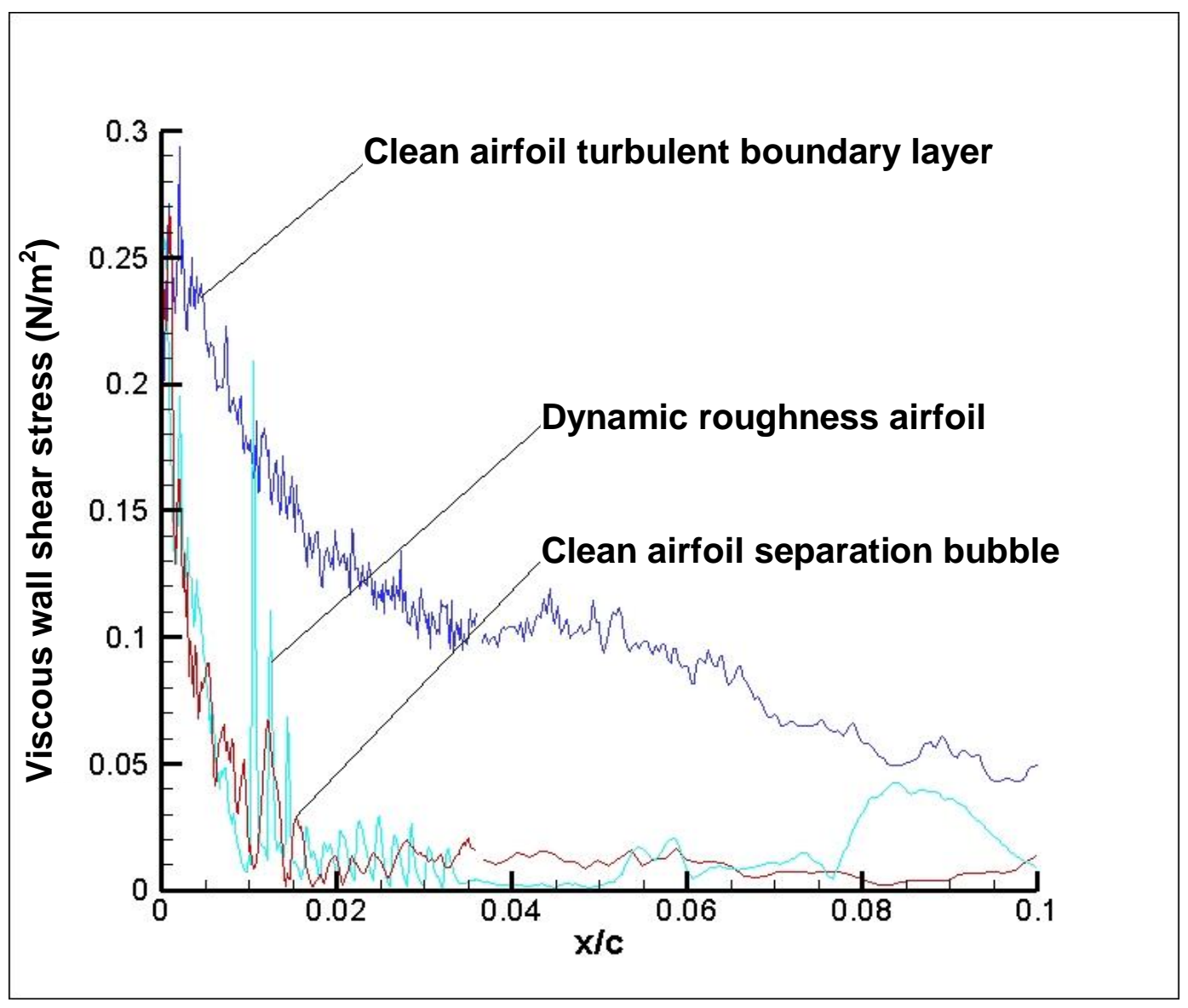

Figure 5.16: Wall shear stress values for clean laminar, clean turbulent, and dynamic roughness laminar wings 
Also of particular interest was the vorticity of the flow near the surface. Vorticity levels were high near the leading edge and dissipated as the flow traveled downstream. As the flow encountered the first hump, the vorticity increased, but as it continued over the remaining humps the vorticity weakened. Figure 5.17 shows the vorticity along with pathlines for two selected humps. It is important to note that this was taken at a particular time step while the humps were in motion. The first figure depicts the first hump in a series of twelve. The second part of the figure shows the last hump in the series. For the first hump, the vorticity is present and the flow passes over the hump attached. On the leeward side of the hump the formation of a vortex can be observed. Due to reasons previously discussed, this vortex tends to remain in this region until it dissipates during the downstroke motion of the hump. The global flow is attached to the surface, but there were these small vortices developed in the valley between roughness elements. In the aft hump region the flow has decelerated but remains attached except for the thin shear layer adjacent to the surface of the hump. 


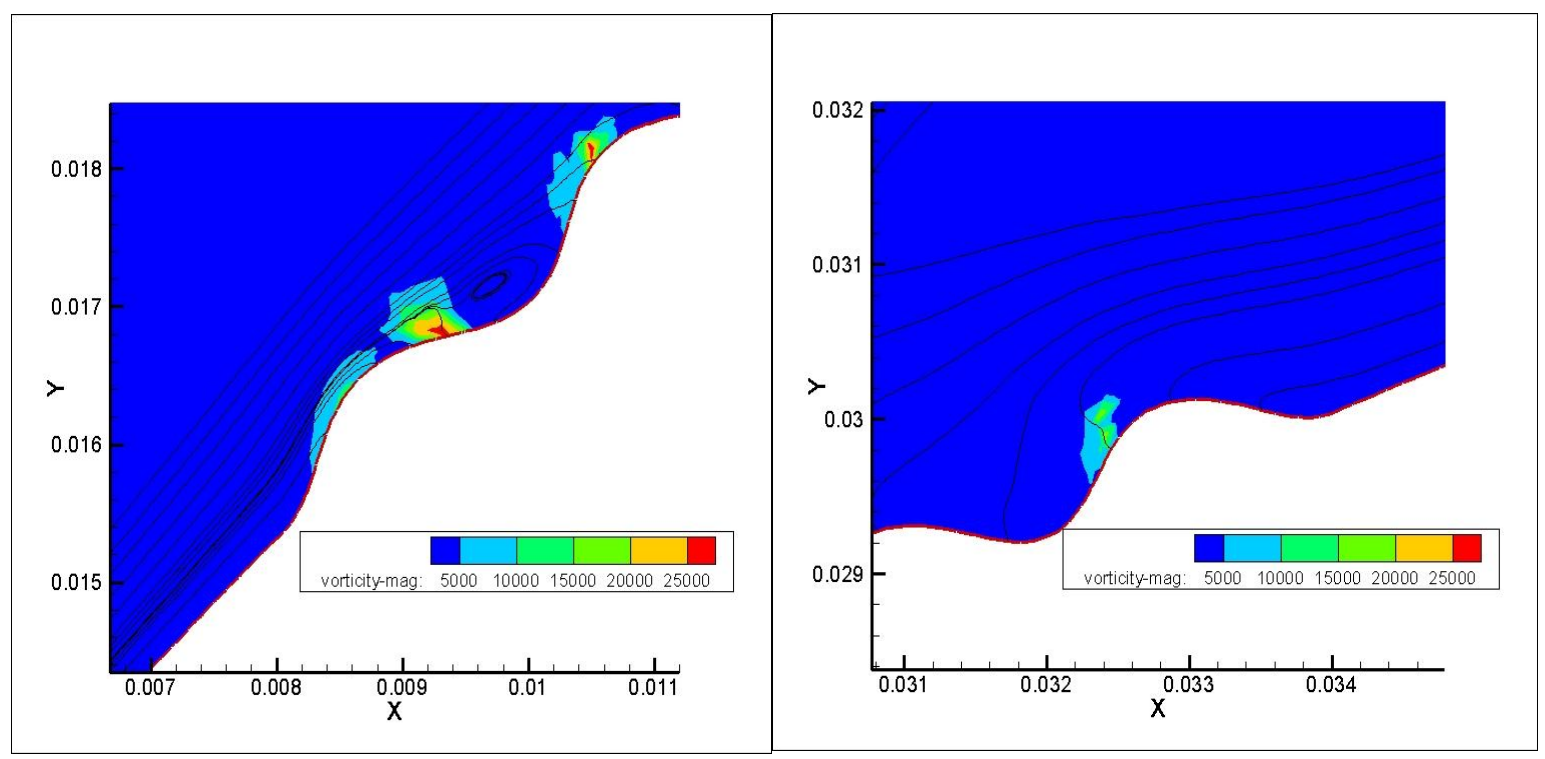

Figure 5.17: Vorticity and pathlines in region of first and last humps during dynamic roughness cycle

\subsection{Comparison of Two-dimensional and Three-dimensional Analysis}

In this research study it was found that the effect of the dynamic roughness did not vary a great deal when analyzing and comparing two and three-dimensional cases. Figure 5.18 shows a selected two-dimensional case. The hump height was $0.8 \mathrm{~mm}$, the angle of attack 12 degrees, and the Reynolds number 100,000. The hump shape was derived from a basic sine wave function. It can be observed in figure 5.18 that the effect of the dynamic roughness in the two-dimensional case is very similar to the three-dimensional case. For future parametric studies, it may suffice to run a majority of the simulations with the twodimensional model. 


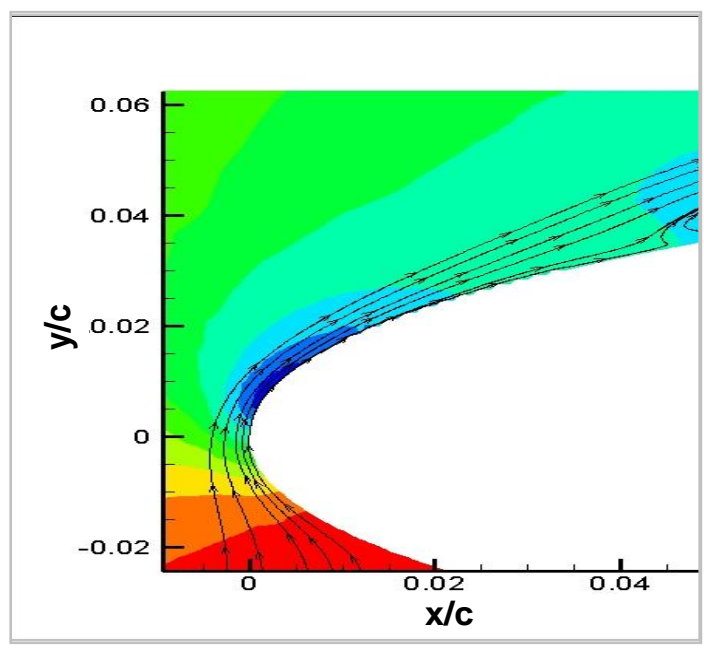

a) Two-dimensional case

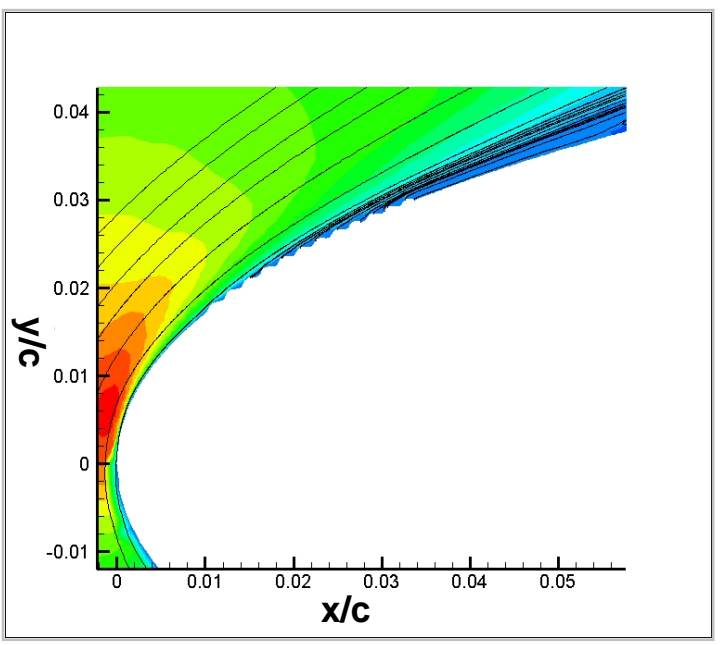

b) Three-dimensional case

Figure 5.18: Two-dimensional and Three-dimensional dynamic roughness cases at 12 degrees angle of attack and 100,000 Reynolds number

\subsection{Amplitude and Frequency Effects of Dynamic Roughness on Flow Control}

Several parametric studies were carried out in order to begin quantifying the effects of amplitude and frequency on the effectiveness of dynamic roughness. Several cases were run for various frequencies and amplitudes ranging from a very shallow roughness height ( $7 \%$ oncoming boundary layer thickness) to a roughness height equal to about $80 \%$ of the boundary layer height and frequencies ranging from $30 \mathrm{~Hz}$ to $120 \mathrm{~Hz}$. When a very small hump amplitude height ( $1-3 \%$ ) was run, it was discovered that the frequency had to be run much higher to obtain flow control. At the higher frequencies, difficulties were encountered in the grid remeshing algorithms, making it difficult to analyze these cases. Numerical results indicated that flow control was obtained with amplitudes as small as $7 \%$ of the oncoming boundary layer height, provided the frequency was high enough. 
The lack of flow control was characterized by the separation point on the leading edge remaining relatively unchanged compared to the clean case (the clean case represents the case of no flow control, the separation point remains fixed near the leading edge). Effective flow control was characterized when the separation bubble was eliminated. The numerical results also indicated a somewhat transient state referred to as a "buffer zone". In these cases, the flow appeared to separate in the roughness region forming a very thin shear layer. However, once passing through the roughness field, the flow then reattached to the airfoil surface. Therefore, this buffer region was a fluctuating state between full control and loss of control. In some cases, at some point beyond the roughness field, the flow would eventually separate. This was believed to be due to the fact that the NavierStokes solver was applied with no turbulence modeling. In actual situations, it is believed that the boundary layer, once leaving the roughness field in a laminarized state, undergoes a natural transition process. The experimental results have qualitatively shown this to be the case.

Figure 5.19 is a summary of the computed flow fields as a function of amplitude and frequency from the CFD results. Each data point represents a separate case where the flow field was analyzed. The green points represent the cases where effective flow control is achieved. Amber indicates the cases where the flow appears to form a very thin shear layer in the roughness region, then reattaches downstream. The red indicates the cases when there is no effective flow control. Also shown is the experimental results, which represent the case where the dynamic roughness height was $0.3 \mathrm{~mm}$ or $20 \%$ of the boundary layer height. There appears to be a correlation between the amplitudes and 
frequencies where flow control was effective. For the case of 12 degrees angle of attack and a Reynolds number of 100,000 this occurs at around $60 \mathrm{~Hz}$. These numerical results are also consistent with the experimental work. As the amplitude of the roughness was decreased it appeared that a higher frequency was required to obtain flow control. Conversely, a larger amplitude required lower frequency to maintain control. This coupling is approximately shown by the sketched buffer zone in the figure. This seems plausible since generating a certain level of artificial Reynolds stresses at lower amplitudes would require higher frequencies of motion. Also, it is significant to note that the roughness Reynolds number is about 120 when the dynamic humps are held at maximum amplitude. This would mimic the case when the dynamic roughness acts a static roughness. The critical roughness Reynolds number normally required for forced transition is about 600 for a two-dimensional airfoil Therefore, in the static roughness analysis, where the humps were held at their maximum amplitude, no effective flow control took place and the laminar separation point remained fixed. 


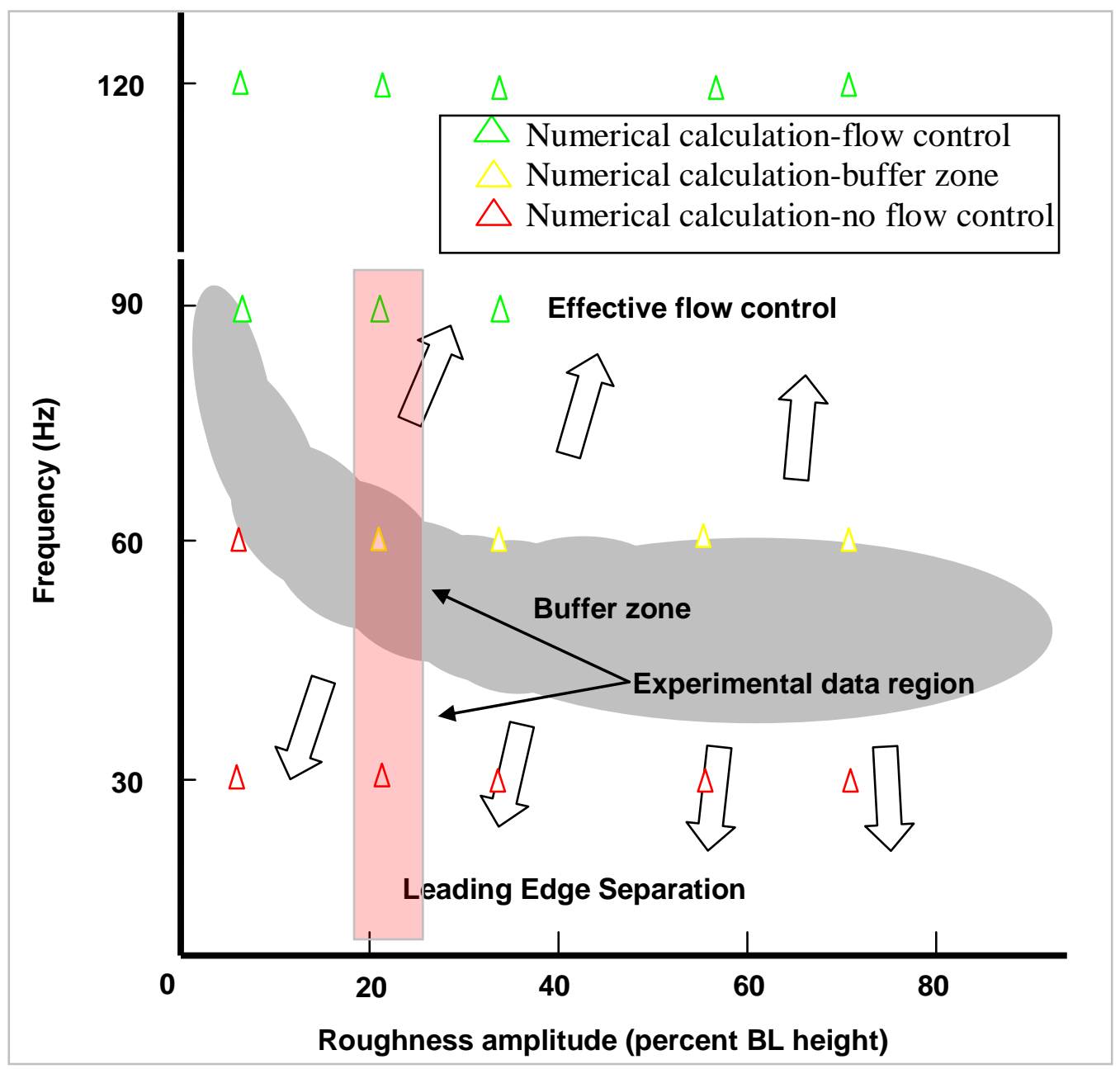

Figure 5.19: Flow control analysis as a function of roughness amplitude and frequency at 12 degrees angle of attack and Re of 100,000

Frequency is often times expressed as a non-dimensional number in the form of the Strouhal number. Figure 5.20 is a plot of the same data shown in figure 5.19 except the frequency has been non-dimensionalized. It was non-dimensionalized by multiplying the frequency times a characteristic length and then dividing by a velocity, as shown in equation 5.1. 


$$
S t=\frac{f l_{x}}{u_{x}}
$$

In this case, the velocity used, $u_{x}$, was the velocity that would exist at a height in the boundary layer which is equivalent to the height of the roughness hump. The characteristic length, $l_{x}$, was the overall flow length of the roughness region. The Strouhal number then represents the frequency of the hump motion divided by the frequency that a given particle would traverse the roughness region. In a sense, it can be looked at as a time scale ratio. The numerator represents the time it would take for a given hump to complete one cycle, and the denominator represents the time it would take for the flow the move from the beginning to the end of the roughness region. A Strouhal number of unity would be the case where as the humps move through one cycle, the flow would pass from the beginning of the roughness field to the end of the roughness field.

As the hump amplitude approaches zero, the frequency approaches an infinite value. Also, as the hump amplitude approaches the boundary layer height, the frequency approaches an asymptotic value and the frequency range becomes smaller. Hump amplitudes greater than the boundary layer thickness were not analyzed for the following reasons; 1) in terms of actuation it is desirable to have small amplitudes of excitation, 2) previous research has shown that amplitudes greater than the boundary layer thickness may not be effective in providing flow control [Huebsch, 2006] and 3) as the hump amplitudes become large, the remeshing algorithms have difficulty redefining the mesh. 


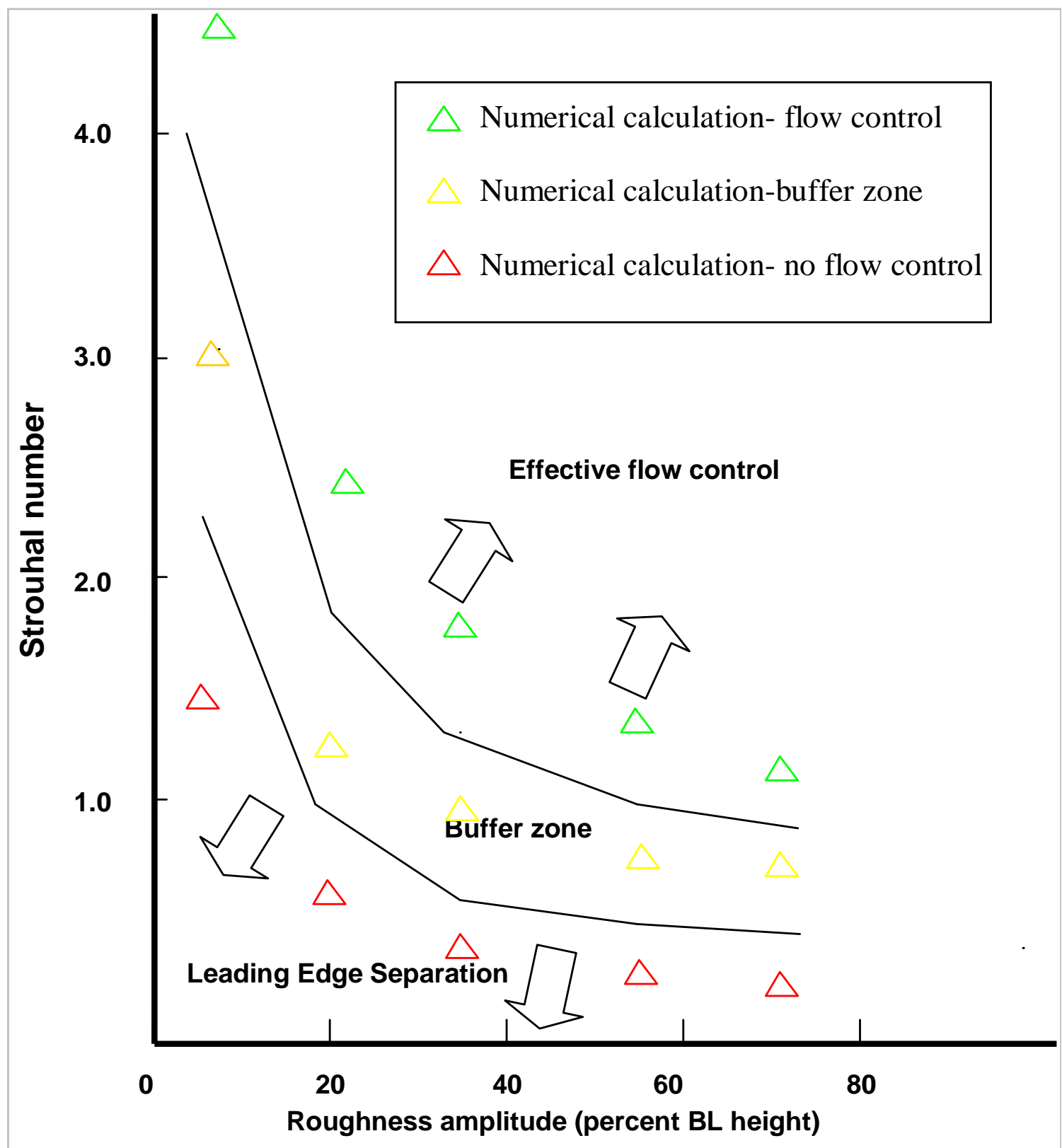

Figure 5.20: Flow control analysis as a function of roughness amplitude and Strouhal number at 12 degrees angle of attack and Re of 100,000 


\section{Chapter 6}

\section{Experimental Analysis of Dynamic Roughness}

In order to experimentally study the effects of dynamic roughness, a wind tunnel model was built and tested in the West Virginia University smoke tunnel. The West Virginia University smoke tunnel is a small tunnel with average to good flow quality and is primarily used for flow visualization. It is an open return type wind tunnel driven with a downstream electric fan drive.

\subsection{Wind Tunnel Description and Set-up}

The wind tunnel inlet consists of a bell mouth entrance which has 8 fine mesh screens installed in it. The screens are intended to reduce the free stream turbulence levels. After passing through the inlet the flow enters a 17.3:1 contraction section before entering the test section. The test section is a square cross section measuring $152 \mathrm{~mm}$ by $152 \mathrm{~mm}$ with see through glass on the top and both sides. It is $356 \mathrm{~mm}$ in flow length. After passing through the test section the flow then enters a diffuser section before finally passing through an electric belt-driven fan and discharged into the open room. The motor drive section mates to the nozzle section but never actually touches it. This creates a slight bit of leakage, but isolates the test section from any motor drive induced vibrations. The photograph in figure 6.1 shows the WVU smoke tunnel set-up. 


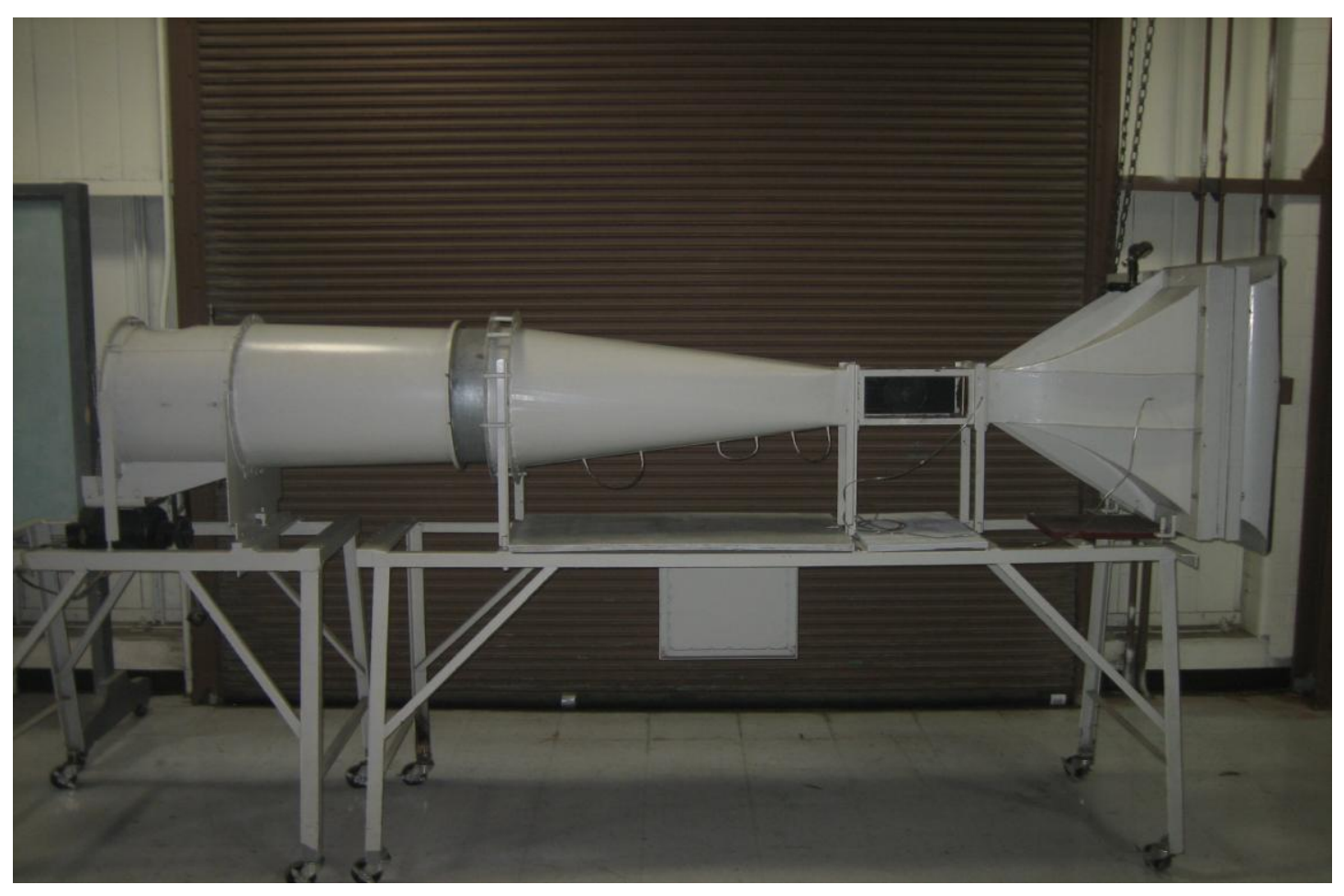

Figure 6.1: Side view of WVU smoke tunnel

Before putting the wind tunnel into operation for this work, a flow survey was performed in order to quantify the flow quality. The wind tunnel velocity is controlled by setting a variac variable $\mathrm{AC}$ controller to a user selected position, ranging from 0 to 10 . The positions are arbitrary and have no relation the actual velocities. The maximum velocity of the tunnel was found to be 23 meters per second. For velocity calibration and determination, there are static port pressure taps located in the test section as well as in the contraction chamber ahead of the point where the area restriction begins. These are designed to measure static pressure values to be used in determining test section velocities. During the initial survey of velocity, pressure measurements were taken at the static ports as well as with a pitot-static tube located in the test section. A sweep of the pitot-static tube was done by transversing it across the test section in $1 / 4$ inch increments. 
The survey showed the velocity to be relatively constant across the test section $( \pm 1 \%)$. Figure 6.2 is a plot of the measured test section velocities at four variac settings, 3, 5, 7 and 9.

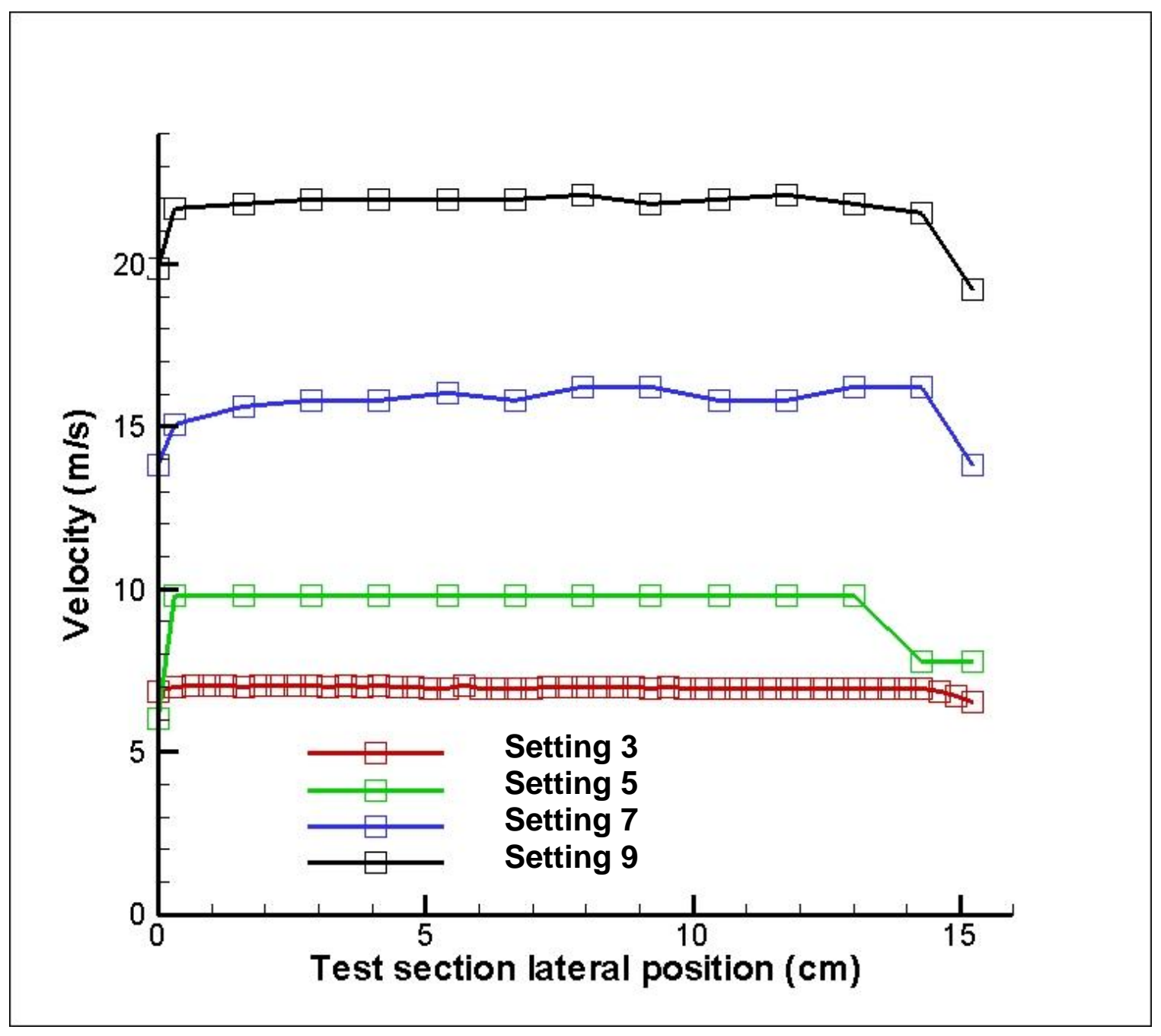

Figure 6.2: Wind tunnel test section velocity profiles for four different velocity settings

The next step was to survey the turbulence levels in the test section. This was done using a hot wire anemometer, specifically the Trust Science Innovation Model IFA 300 constant temperature hot-wire anemometer system. Turbulence measurements were 
taken at three different lateral locations across the test section approximating the $25 \%$, $50 \%$ and $75 \%$ lateral span locations. Results indicated the turbulence levels to be quite interesting. At the lowest tunnel setting of 3 , which results in a velocity of approximately 6.7 meters per second, the turbulence intensity was about $2 \%$. As the tunnel velocity was increased to 9.7 meters per second the turbulence levels dropped dramatically to levels of about $0.2 \%$. As the tunnel speed was increased further to 16.0 meters per second the turbulence levels increased to levels of about $5 \%$. The turbulence tests were repeated and yielded consistent results. There appears to be a correlation between the turbulence levels and the test section velocity. The more uniform the velocity profile, the less the turbulence levels. It was undetermined why the test section velocity seemed to provide the best flow at mid-range settings. A plot of the turbulence values is shown in figure 6.3. 


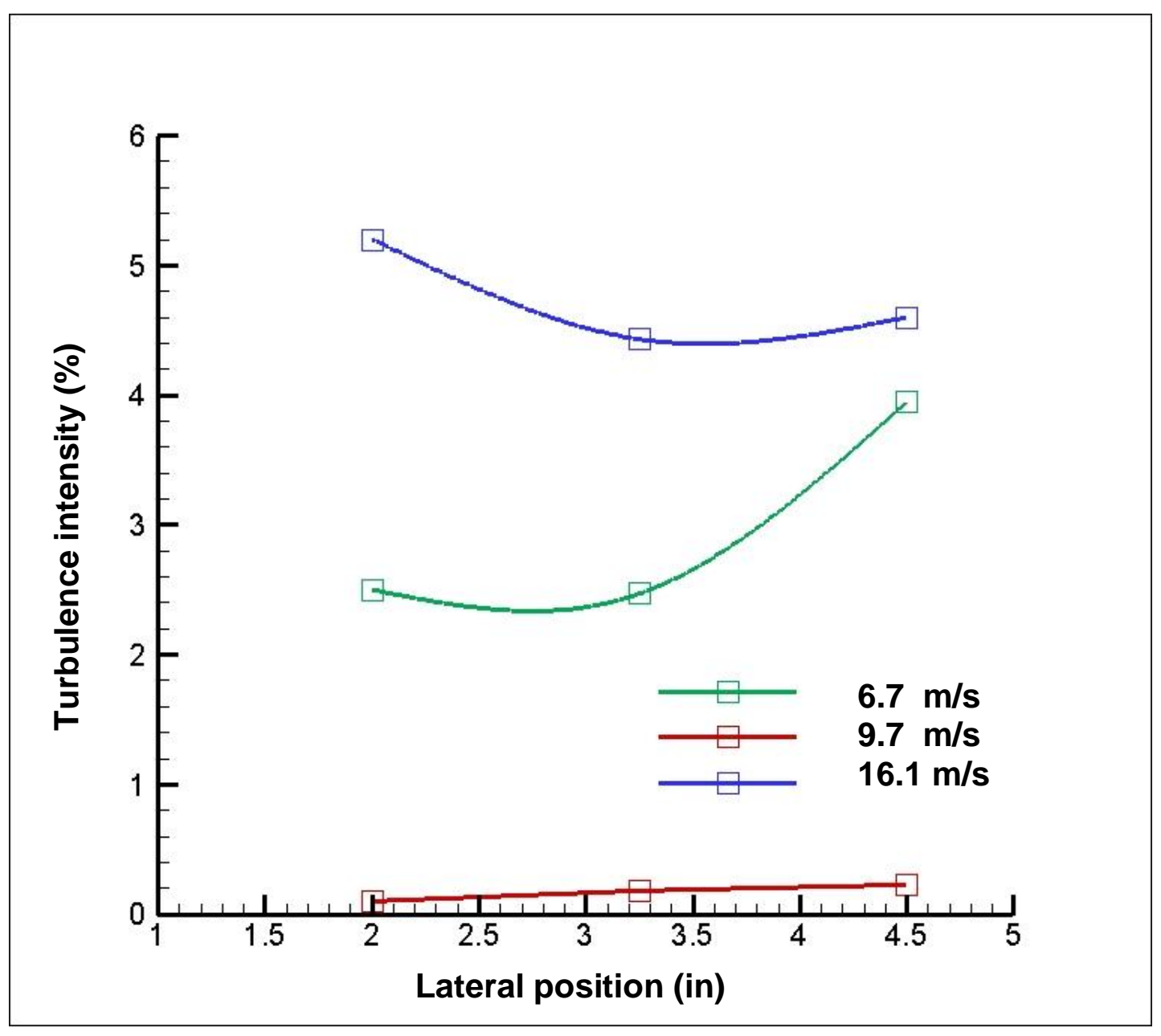

Figure 6.3: Wind tunnel turbulence measurements

\subsection{Dynamic Roughness Wind Tunnel Model}

A dynamic roughness wing was designed and constructed for testing in the smoke tunnel. The wind tunnel model was fabricated by Wilson Works Co from steel plating in accordance with provided specified drawings. Table 6.1 lists the specifications of the fabricated model. The basic model substructure and internal baffling was fabricated from 22 gauge steel. A "pocket" in the shape of a channel was also designed and fabricated into the upper surface so that the dynamic roughness apparatus could be assembled and 
fit into this channel. The model is designed such that the dynamic roughness apparatus itself is fabricated into an integral part which can be installed and removed from the pocket.

Table 6.1: Wind tunnel model specifications

\begin{tabular}{|c|c|}
\hline Airfoil & NACA 0012 \\
\hline Chord & $151 \mathrm{~mm}$ \\
\hline Span & $152 \mathrm{~mm}$ \\
\hline Max thickness & $18.1 \mathrm{~mm}$ \\
\hline
\end{tabular}

This allowed for the testing of various types of mechanisms that may be used to actuate the dynamic roughness. Figure 6.4 shows the model substructure.

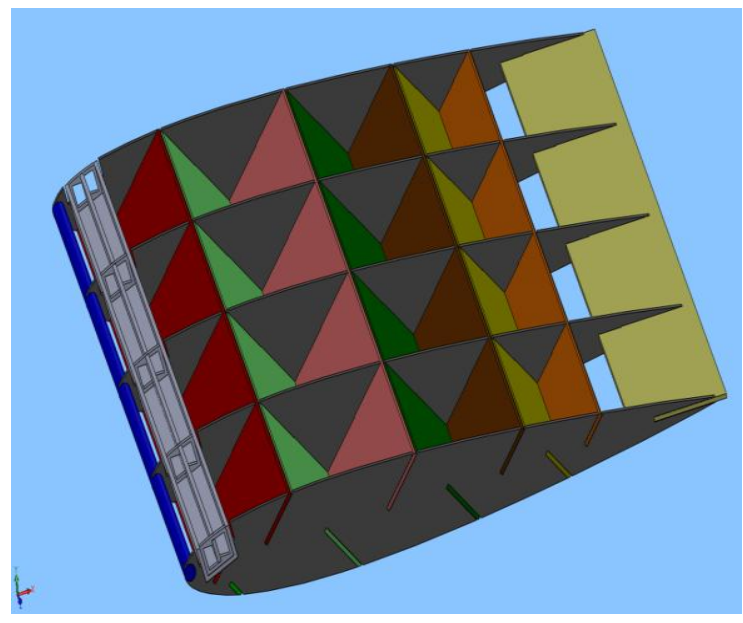

a) Assembly without airfoil surface

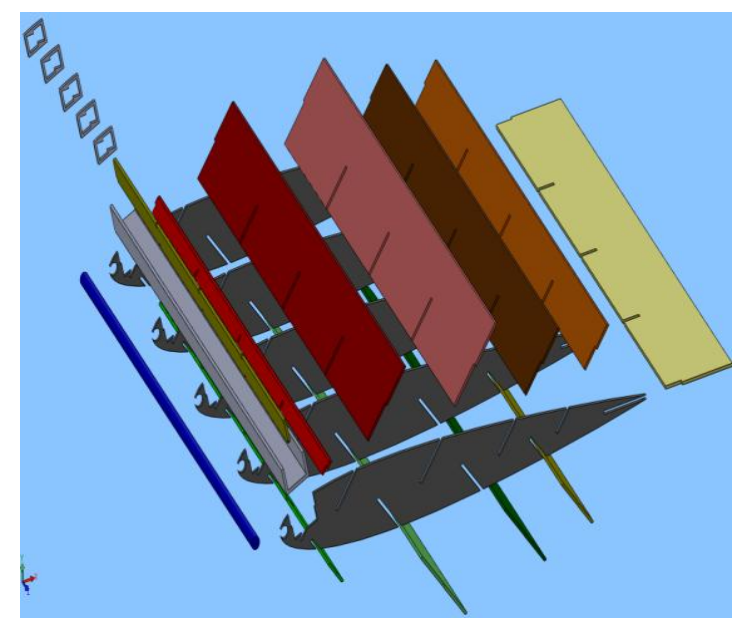

b) Exploded assembly view 


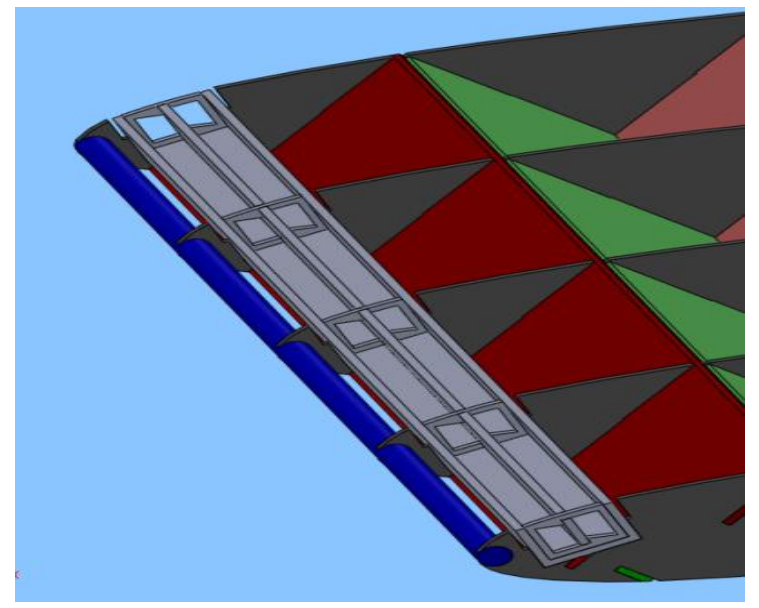

\section{c) Depiction of dynamic roughness apparatus in leading edge region}

\section{Figure 6.4: Dynamic roughness model substructure}

In order to incorporate the dynamic roughness, an apparatus had to be constructed. It was decided that for the 1 st generation model it would be easiest to actuate the dynamic roughness through oscillating air pressure and a flexible surface constrained to produce roughness elements. This proved to be a tedious process. Construction began by starting with a thin wire mesh. The wire mesh consisted of diamond shaped areas. The first step was to bond a very thin rubber latex sheet over the wire mesh, which would be the flexible surface to produce the roughness. This was accomplished by applying a thin coating of CA cement to the mesh, and then laying the latex rubber over the mesh. Great care had to be taken when accomplishing this step. If too much cement was applied, the cement will coat the latex and freeze it in place, not allowing it to flex when pressure is applied. If the cement was applied to soon, it would set up before bonding can take place. Once the latex is correctly bonded to the mesh, it was then cut to the correct dimensions and cemented to the aluminum $\mathrm{C}$ channel. Again this had to be carefully accomplished in 
order to get an airtight seal. Once this was completed, a nylon nipple (sized for 1/4 inch tubing) was fit into one end of the channel and glued into place. The other end was merely capped to seal the chamber. If successful, the apparatus should provide an airtight chamber such that when the oscillating air pressure is applied, the latex will expand and contract, mimicking the geometry of a series of dynamic humps. Figure 6.5 is a photo of the completed apparatus ready for installation into the airfoil model. Figure 6.6 shows the apparatus when a fluctuating air pressure source is applied. In figure 6.6(a) the humps are flush with the surface. In figure 6.6(b) the humps can clearly be seen extended to their maximum amplitude position.

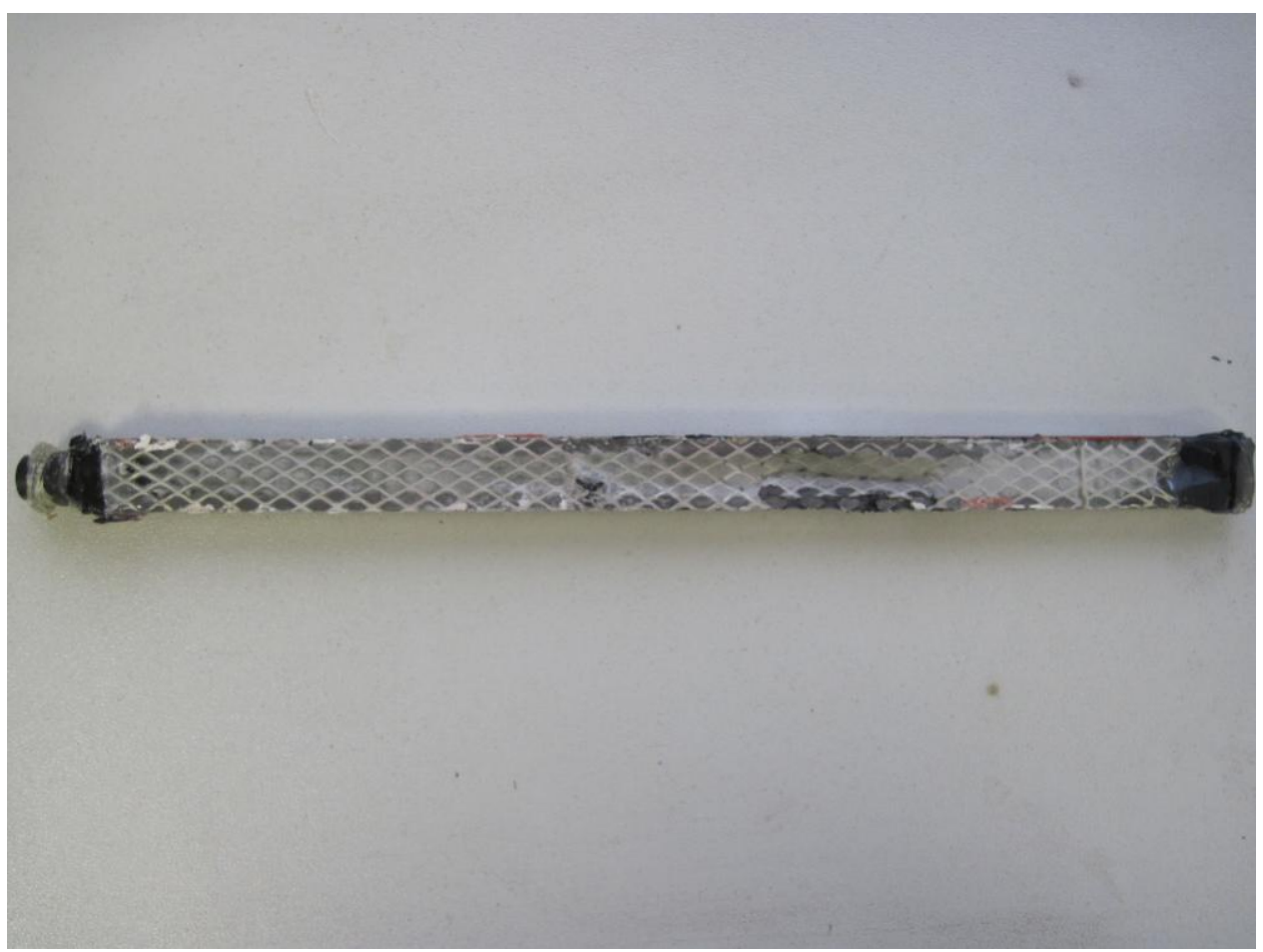

Figure 6.5: Completed dynamic roughness apparatus 


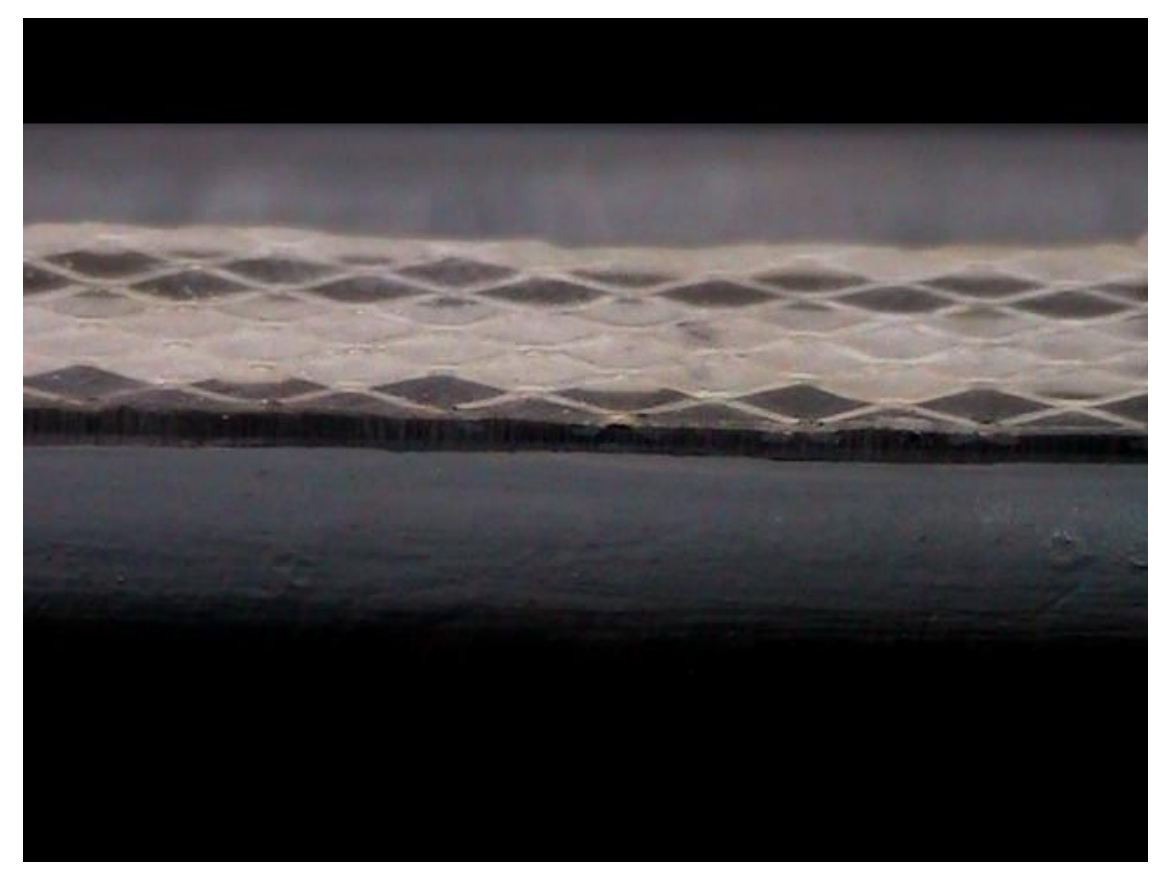

a) Humps flush with surface

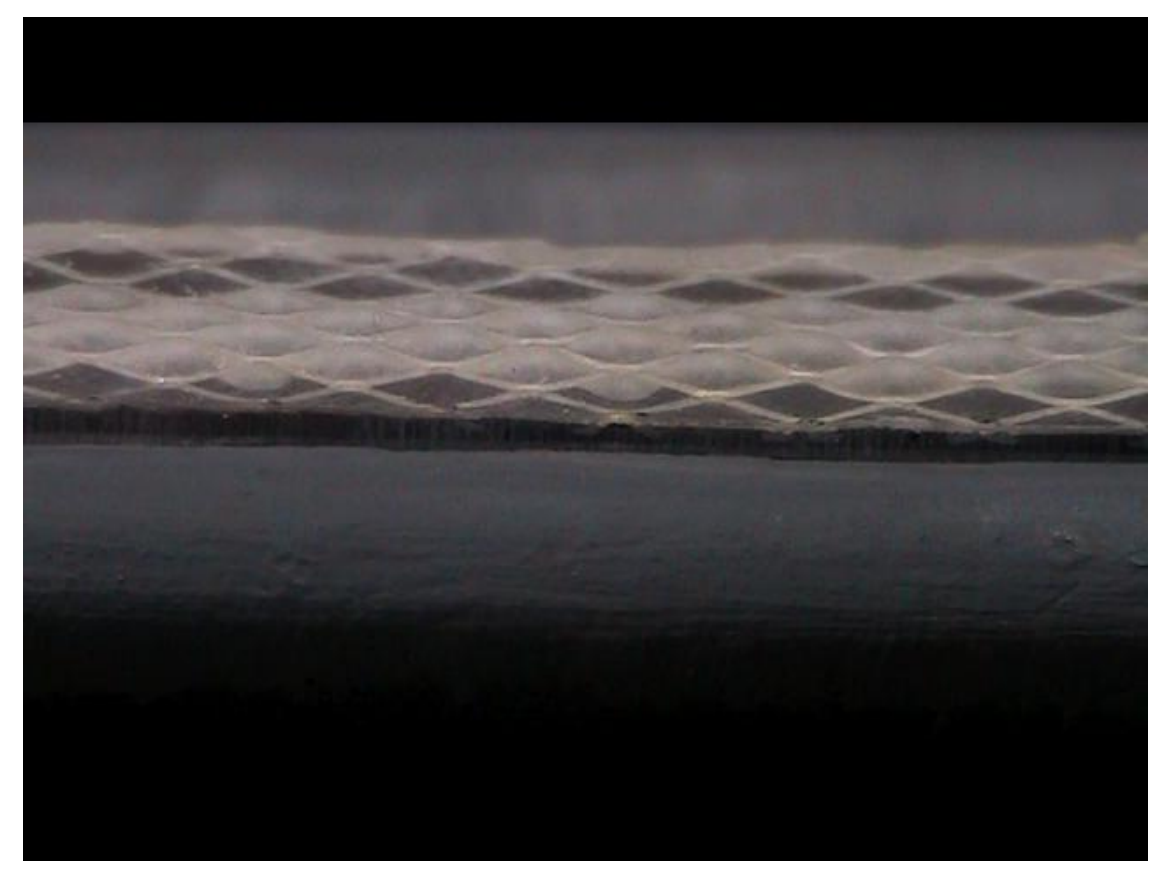

b) Humps extended

Figure 6.6: Dynamic roughness apparatus with oscillating pressure applied 
At this point the apparatus was inserted into the surface of the airfoil where dynamic roughness could be simulated. This dynamic roughness covered the full span and ranged from $3.1 \%$ chord to $7.8 \%$ chord. A tube connected the dynamic roughness chamber to a small piston cylinder type arrangement. The piston cylinder was driven by a variable speed motor through a 1:4 gear ratio increase. The RPM of the motor could be directly correlated to the frequency of the roughness motion. This was confirmed by conducting a test in which the piston-cylinder was operated at a given frequency and a stroboscope was used to verify that the roughness was oscillating at the same frequency as the pistoncylinder. This was done on the model outside of the wind tunnel. The stroboscope, being intended for automotive use, required a distributor connection in order to change the trigger rate. The default setting was 1200 RPM, which when applied to the test apparatus corresponded to a piston-cylinder frequency of 80 cycles per second. At this setting the humps did oscillate at $80 \mathrm{~Hz}$ with an amplitude of about $0.3 \mathrm{~mm}$. The maximum motor RPM was approximately 2200 RPM which translated to a dynamic roughness frequency of about 147 Hertz. The amplitude of the dynamic roughness, based on photographs of the motion, appeared to be about $0.3 \mathrm{~mm}$. The final assembly of the wind tunnel model and a close up view of the dynamic roughness apparatus in place is shown in figure 6.7 


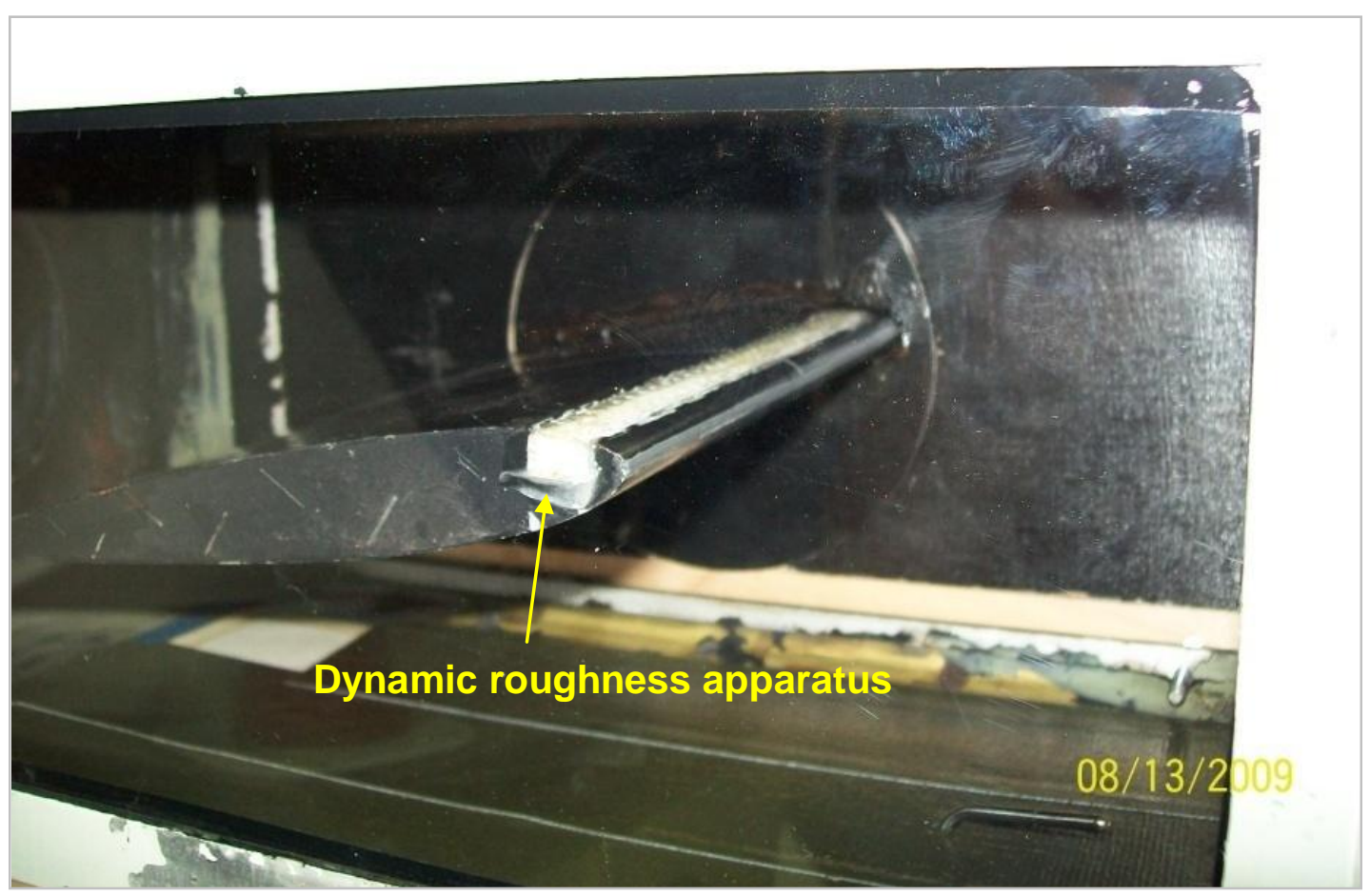

a) Dynamic roughness model mounted in wind tunnel

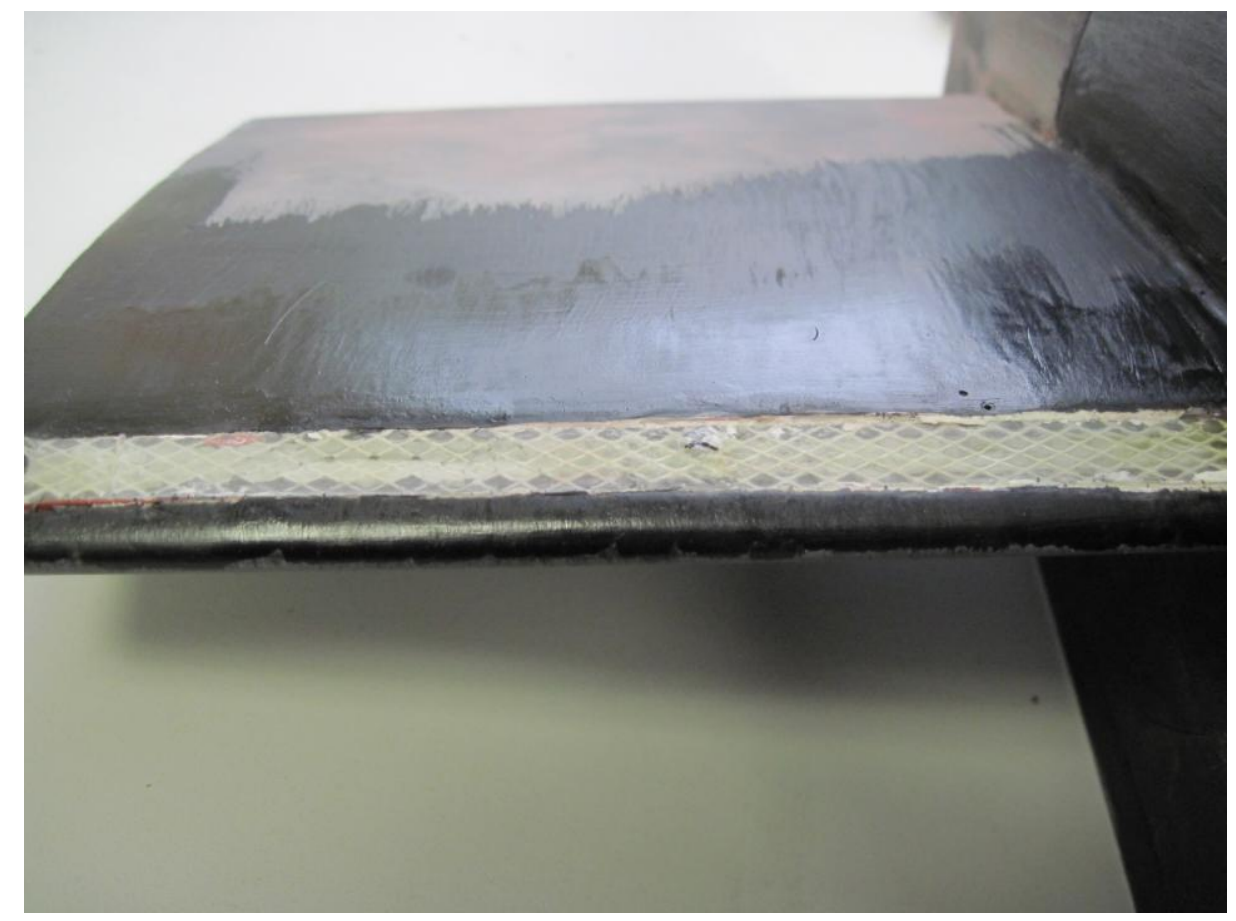

b) Front view of model and dynamic roughness 


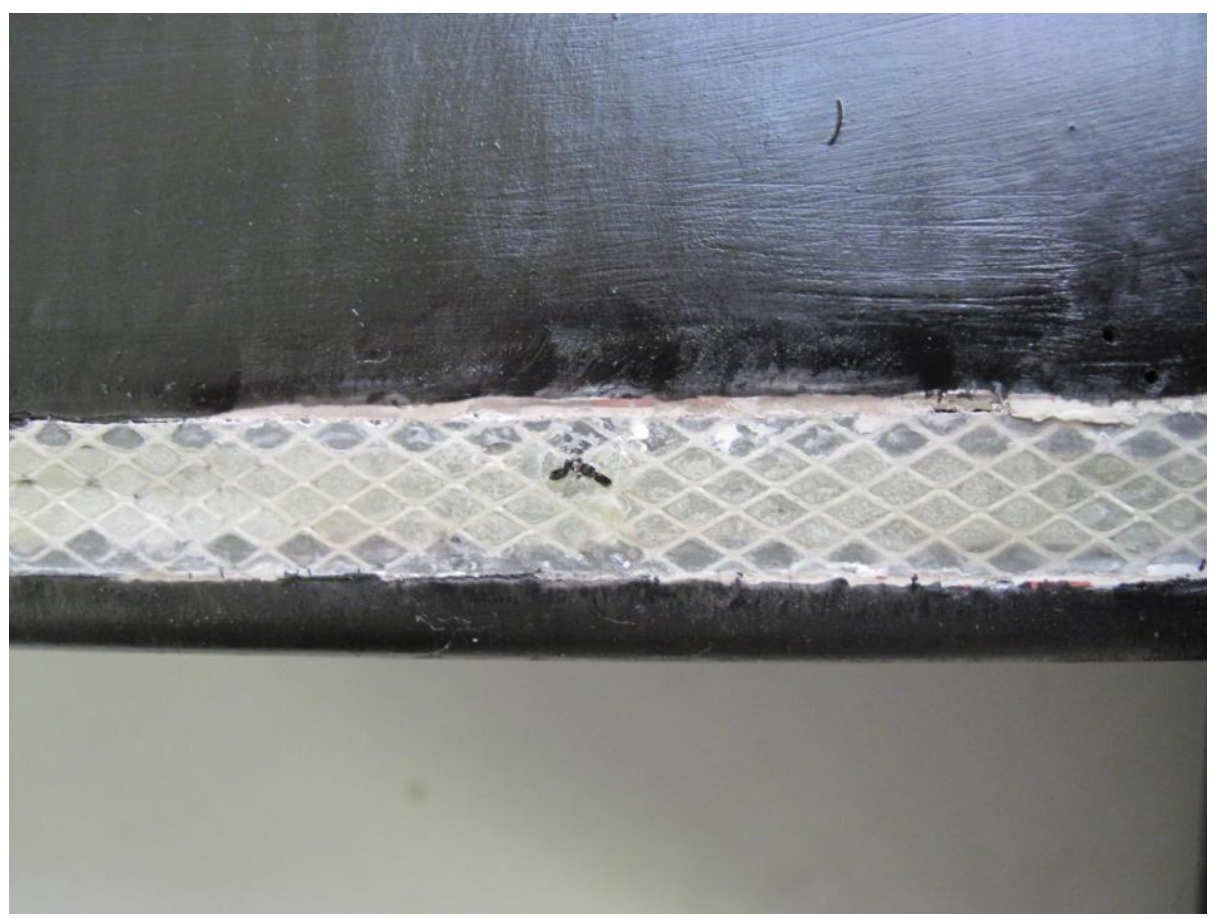

c) Close-up view of dynamic roughness apparatus

Figure 6.7: Final assembly of dynamic roughness model

Other mechanisms have been proposed and attempted as a means of creating an apparatus which could create dynamic roughness. One attempt involved using a speaker to create the oscillating pressure source. The speaker was driven at selected frequencies using a signal generator and amplifier. The open end of the speaker was sealed in a chamber such that actuation of the speaker would cause the pressure to oscillate in the chamber. An air tube then connected the chamber to the apparatus. It was discovered that the speaker did not produce a high enough pressure to actuate the roughness, even though the signal amplifier was increased to the maximum output at which the speaker was rated $(150 \mathrm{~W})$. 
Another method involved using a solenoid valve to oscillate the pressure. The solenoid valve was also driven by a signal generator. It was discovered that the ports within the solenoid valve were to small to allow enough volume of air to pass through with each pressure change. Therefore, this was ineffective in actuating the dynamic roughness.

\subsection{Airfoil Pressure Measurements}

In order to collect airfoil pressure data, several flush static pressure orifices were fabricated into the model. Since this was added after the model was constructed, the pressure taps had to be inserted into the model from the side. The pressure taps consisted of $1 / 16$ inch copper tubing. The pressure taps allowed pressure reading to be taken at 5 chordwise locations for the clean airfoil and unfortunately only two chordwise locations when the dynamic roughness apparatus was in place. Figure 6.8 is a photograph of the clean airfoil showing the pressure taps installed. This photograph was of a model which was fabricated after testing to illustrate the pressure tap installation. In the actual test apparatus the tap installation was much improved.

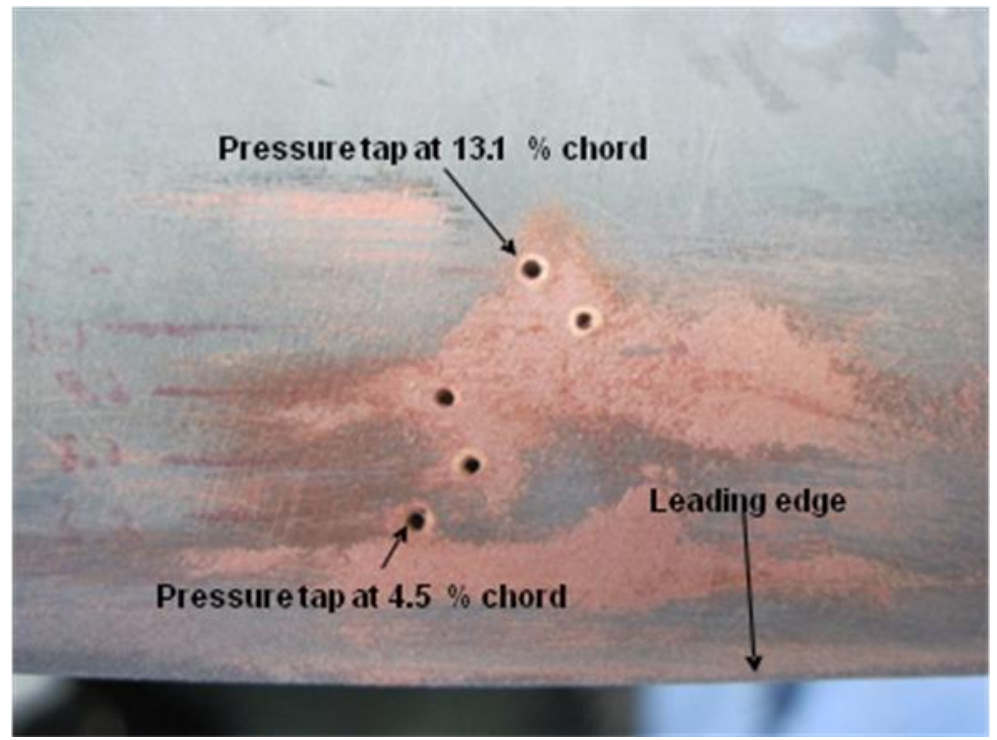

Figure 6.8: Pressure taps on model 


\subsection{Flow Visualization}

In order to visualize the flow two smoke systems were utilized. The first system consisted of creating the smoke with a commercial theater type smoke generator. The second system was a system designed and built by the author along with the assistance of several engineers at NASA Langley Research Center.

The first system to be used was a modified commercial smoke generator. The smoke, after leaving the generator, passes into a plenum chamber of approximately 0.06 cubic meters. Here it was kept warm so that it did not immediately condense. The smoke left the plenum and then passed through a rubber tube into the discharge rake. The discharge rake is located inside the tunnel just downstream of the bell mouth entrance. Figure 6.9 is a photo of this system and figure 6.10 is a view of the smoke discharge rake located at the entrance of the tunnel. A drawback of this system was that it produces no pressure head. Therefore the system relied on a lower pressure at the discharge rake to draw the smoke through the system.

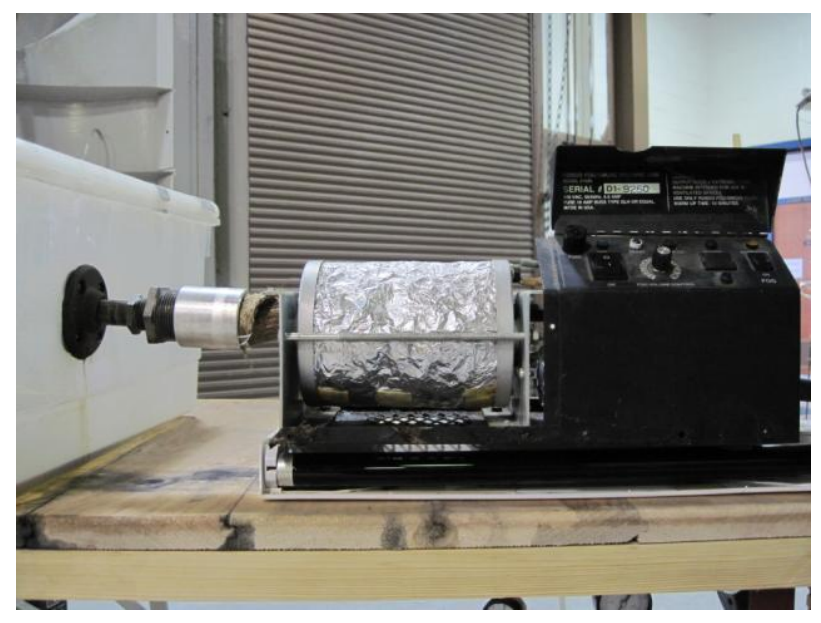

Figure 6.9: Modified commercial smoke generation system 


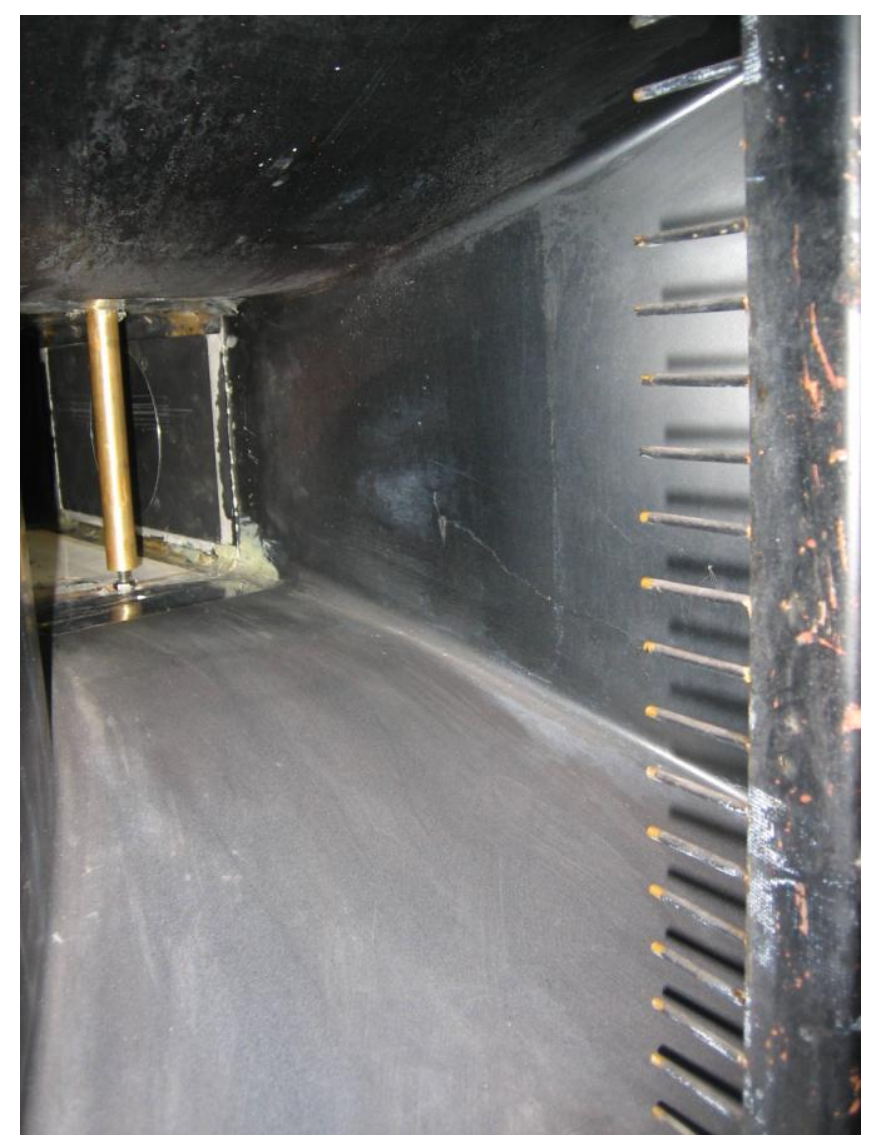

Figure 6. 10: Smoke discharge rake

The second smoke generation system is a unique system built by the author. The potential problem with the first system was that it could not discharge smoke with any significant pressure head. This system was designed to overcome that problem. An illustration of the system is shown in figure 6.11. The smoke oil was discharged through a needle valve and immediately mixed with low pressure air. Typical operating air pressures were on the order of less than one psi, but the pressure could be regulated to a user desired level. Once the oil was mixed with the air the mixture then flowed through an electrically heated tube. The heating process was monitored by thermocouples and controlled such that the oil-air mixture was maintained at a desired set temperature. Experience proved that heating the mixture to a temperature of 540 degrees Fahrenheit provided dense 
smoke. It was critical that the mixture never reach the flash point, about 980 degrees for the propylene glycol mixture. After being heated the oil then flowed into a small plenum chamber before being redirected into the appropriate discharge orifice. Tests showed that the plenum chamber allowed the smoke mixture to cool slightly, which tended to thicken the density of the smoke. Figure 6.12 shows a portion of the system where the oil and air were mixed, and then passed into the heated section of tubing.

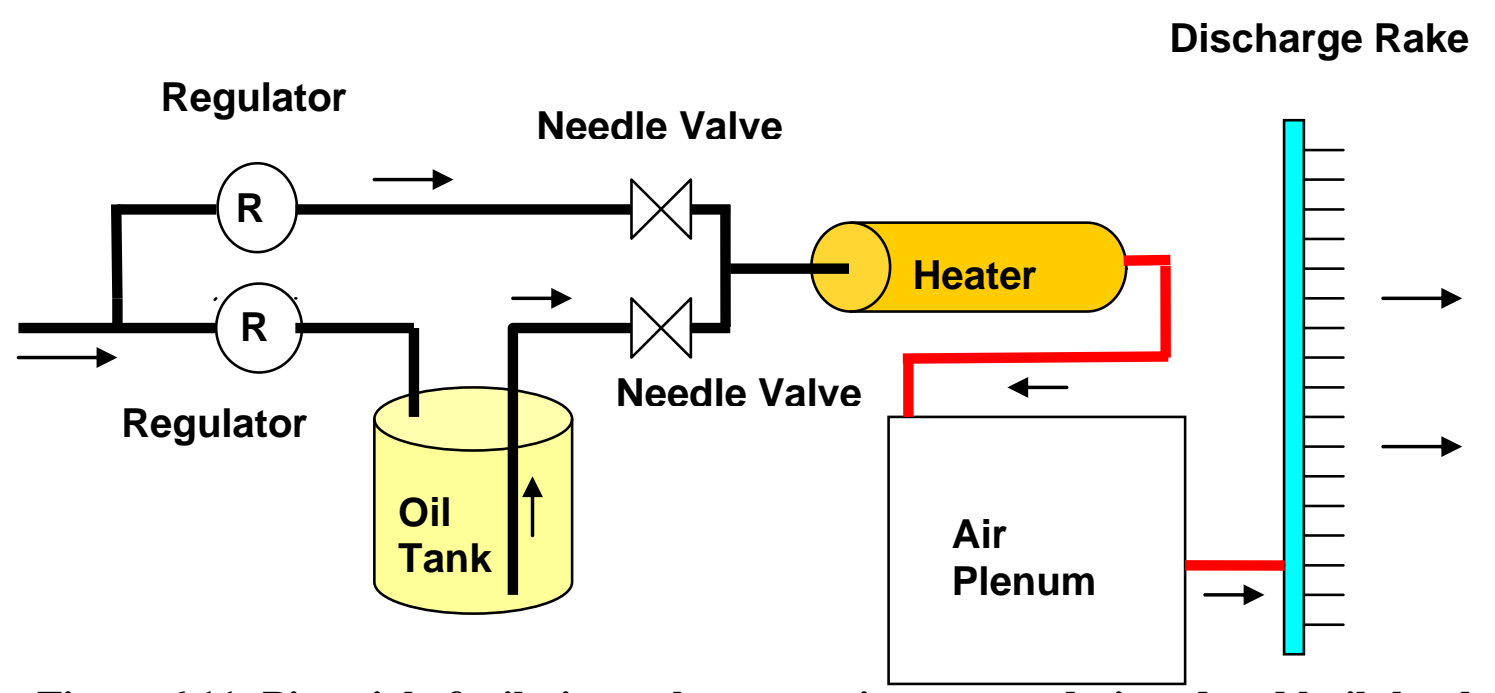

Figure 6.11: Pictorial of oil-air smoke generation system designed and built by the author 


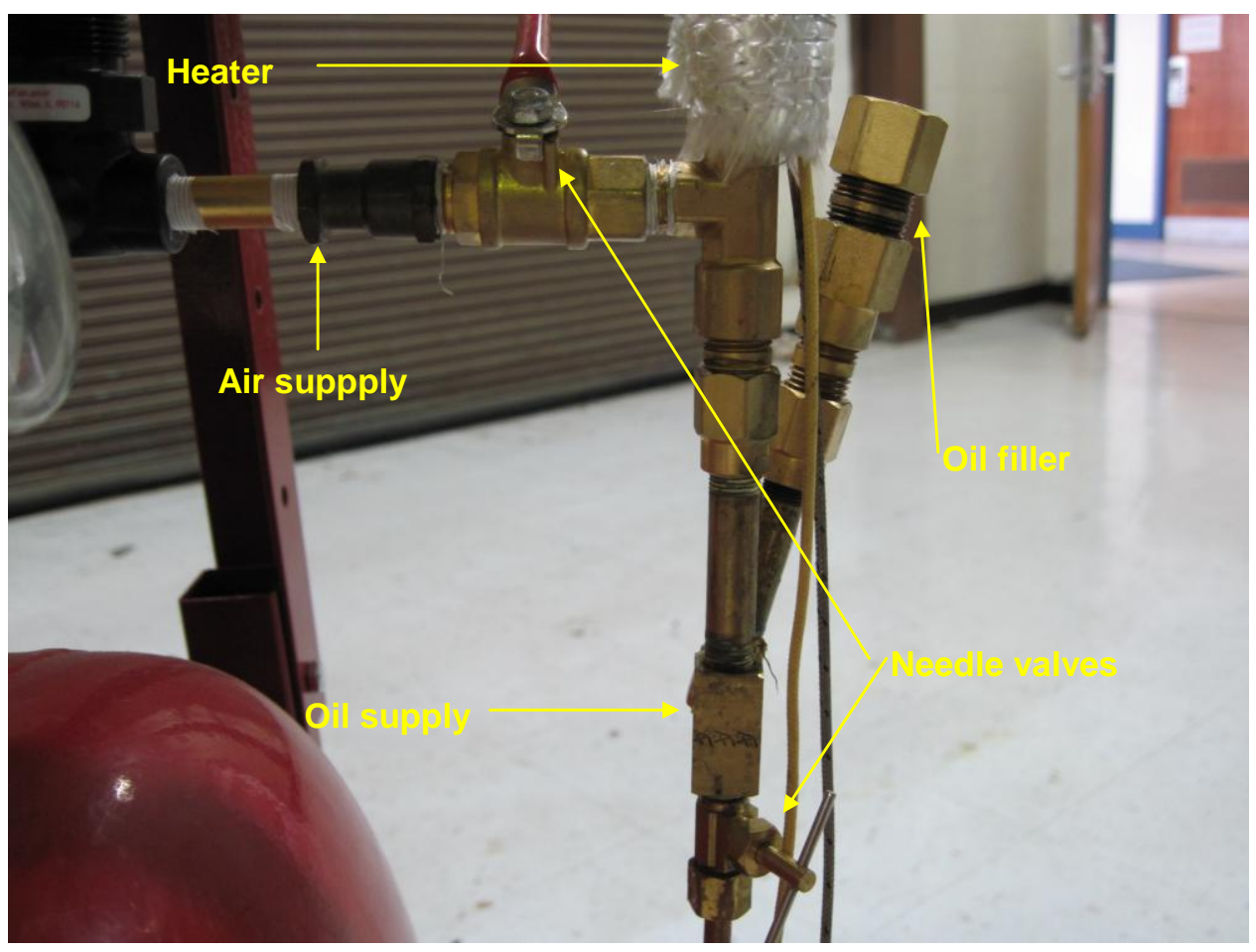

Figure 6.12: Oil-air smoke generation system 


\section{Chapter 7}

\section{Comparison of Experimental and Numerical Results}

The primary goal of this research was to numerically predict the possibility of implementing dynamic roughness to delay and/or eliminate flow separation and to experimentally validate this concept. This was successfully done for both the short and long leading edge separation bubbles. Flow visualization and surface pressure measurements were used to analyze the experimental results.

\subsection{Clean Airfoil Separation}

For the first phase of the experiment, the clean airfoil was tested at two different velocities resulting in two different chord Reynolds numbers of 100,000 and 150,000. For the clean airfoil tests, the dynamic roughness apparatus was removed and the cavity was faired in with basswood such that the surface was smooth and pressure ports could be installed. Table 7.1 outlines the parameters used in the wind tunnel testing.

The effective Reynolds number is sometimes used when tests are conducted in a wind tunnel in which free stream turbulence is present. This effective Reynolds number is somewhat greater than the Reynolds number calculated using the free stream test section velocity and model dimensions. It is due to turbulence in the test section. It is arrived at by placing a sphere in the test section and noting the Reynolds number at which the drag levels change due to the boundary layer transitioning to turbulent prior to separation. This Reynolds number is then compared to the Reynolds number which would exist if no 
turbulence were present. The ratio of these numbers is known as the turbulence factor. In this experiment, the turbulence factor was arrived at by meaduring the turbulence intensity and then using the charted data in Dryden and Kuethe, 1929 to arrive at the effective Reynolds number. At the lower Reynolds number of 100,000 the turbulence intensity was $0.2 \%$ which results in a turbulence factor of 1.2 [Dryden and Kuethe, 1929]. This equates to an effective Reynolds number of 120,000. At the higher test Reynolds number of 150,000 the turbulence intensity was $2.5 \%$ and the resulting turbulence factor was 1.8. This equates to an effective Reynolds number of 270,000. The flow visualization shown in this report was done at the lower Reynolds number of 100,000 or an effective Reynolds number of 120,000 . Flow visualization at the higher Reynolds number of 150,000 was difficult due to the smoke lines having poor resolution.

The goal of this phase was to evaluate and document the separation bubble behavior at various angles of attack and correlate the data with past experimental results. Again, the NACA 0012 airfoil was chosen due to the fact that: 1) there is an abundance of experimental data available on this particular airfoil, including studies on low Reynolds number aerodynamics and separation bubble behaviors and 2) the model does exhibit classic separation bubble type behavior at low Reynolds numbers.

Table 7.1: Wind tunnel test parameters

\begin{tabular}{|c|c|c|c|}
\hline Density & Velocity & Pressure differential & Reynolds number \\
\hline $1.142 \mathrm{~kg} / \mathrm{m}^{3}$ & $11.70 \mathrm{~m} / \mathrm{s}$ & 0.312 in $\mathrm{H}_{2} \mathrm{O}$ & 100,000 \\
\hline $1.142 \mathrm{~kg} / \mathrm{m}^{3}$ & $17.59 \mathrm{~m} / \mathrm{s}$ & 0.704 in $\mathrm{H}_{2} \mathrm{O}$ & 150,000 \\
\hline
\end{tabular}


Before discussing the results of this study, it is worthwhile to review past research data obtained for this particular airfoil. A careful study of the NACA 0012 airfoil lift versus angle of attack curves at various Reynolds numbers can help lead the way in explaining airfoil separation behavior. Figure 7.1 is a plot of the lift coefficient versus angle of attack for the NACA 0012 airfoil taken at both a low Reynolds number of 100,000 [Jacobs, 1938] and a higher Reynolds number of 3,000,000 [Abbot and Von Doennoff, 1956]. At a Reynolds number of $3,000,000$ it is unlikely that a leading edge separation bubble phenomenon exists. The lift curve remains linear up until the point where the stall is approached and the slope approaches the theoretical value of 6.28 per radian or 0.109 per degree. At approximately 12 degrees angle of attack a classic trailing edge type stall resulting from trailing edge separation begins to take place.

Next one can examine the much lower Reynolds number case of 100,000. In this regime a leading edge separation bubble is known to form and exist. This becomes evident when one examines the lift curve at about 6 degrees angle of attack. At this point, the lift curve becomes non-linear. This is due to the fact that the separation bubble has began forming on the leading edge and is resulting in a slight reduction in lift. As the angle of attack is further increased, the separation bubble grows and the adverse impact on the lift becomes more pronounced. Finally a point is reached where the bubble bursts and significant lift is lost. This occurs at an angle of attack of about 10 degrees which is much sooner then the stall angle of attack for the higher Reynolds numbers. 


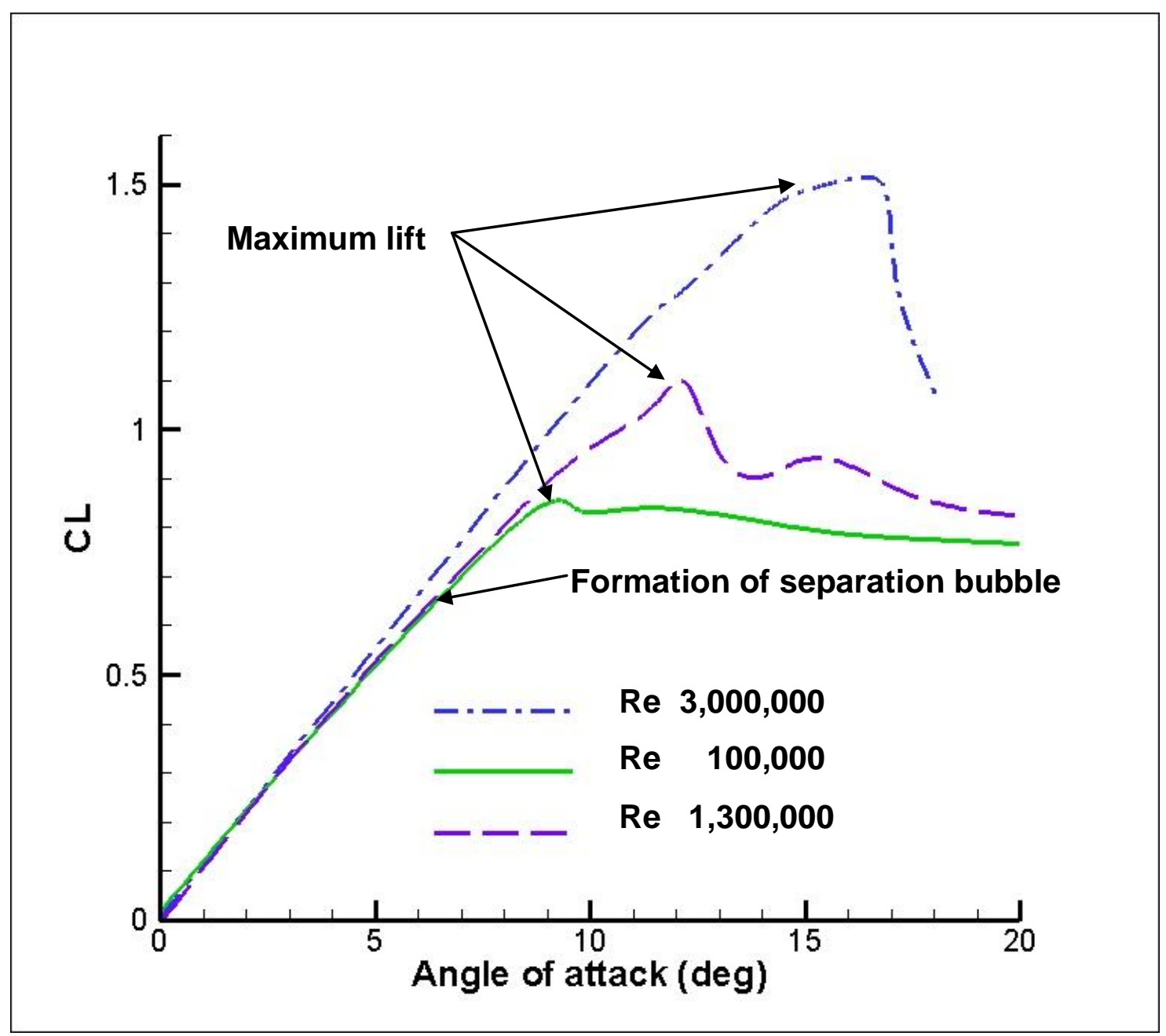

Figure 7.1: Lift curve slope for NACA 0012 at Reynolds numbers of 100,000, 1,300,000 and 3,000,000 [Abbot and Doenhoff, 1956 and Jacobs, 1938]

For the experimental study, an angle of attack of 12 degrees was examined. This was done to evaluate the dynamic roughness at angles of attack above the maximum lift angle of attack in order to study the effectiveness of the dynamic roughness on recovering the loss of post stall suction pressure. Results from the current study can be used to validate the lift curve behavior in figure 7.1. Figure 7.2 shows the flow visualization patterns of the airfoil at selected angles of attack. At 6 degrees angle of attack it appears that a short bubble does form. This bubble continues to exist until an angle of attack of about 10 degrees is reached, at which point the bubble bursts and transforms itself into a long 
bubble. The bubble is clearly visible when tested at the lower velocity and Reynolds number of 100,000. However, at the higher velocity and Reynolds number of 150,000 the smoke lines are not nearly as visible when photographed. This is also the velocity levels where the turbulence levels in the tunnel rise dramatically. These results are in close agreement with the results of Rinoi and Takemura [2000] who tested the same airfoil at a Reynolds number of 130,000 . The results of this step proved to be successful and provided a baseline of data points for the model being tested.

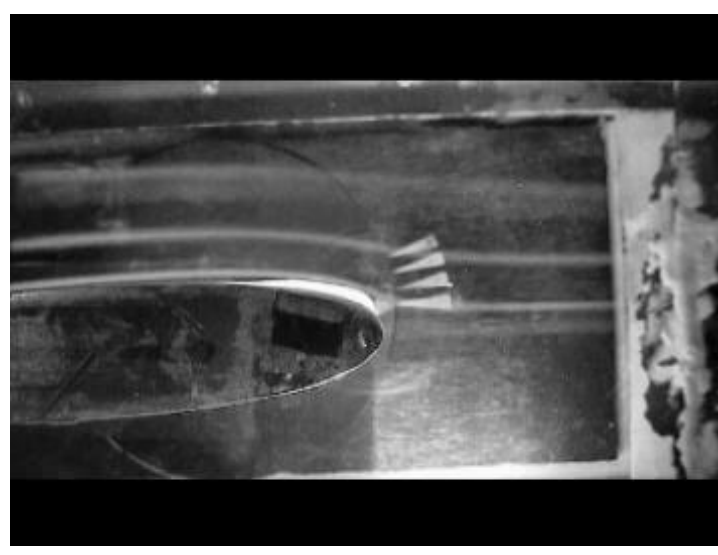

a) Airfoil at 0 degrees angle of attack attack, attached flow

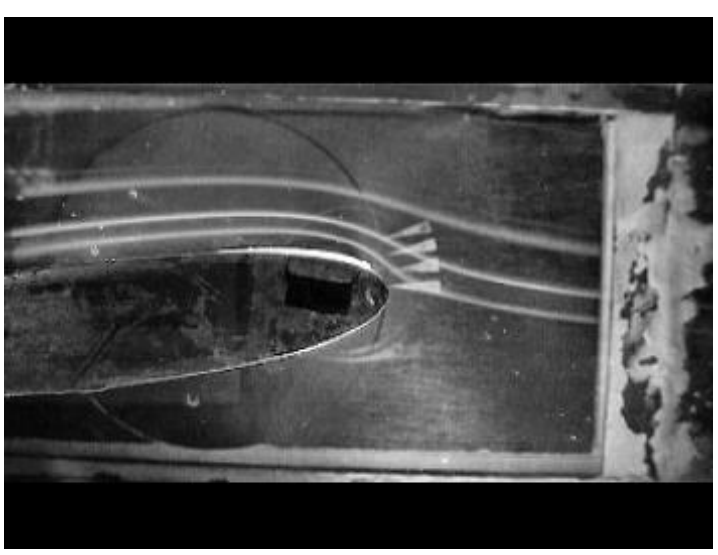

\section{b) Airfoil at 5 degrees angle of attack, attached flow}




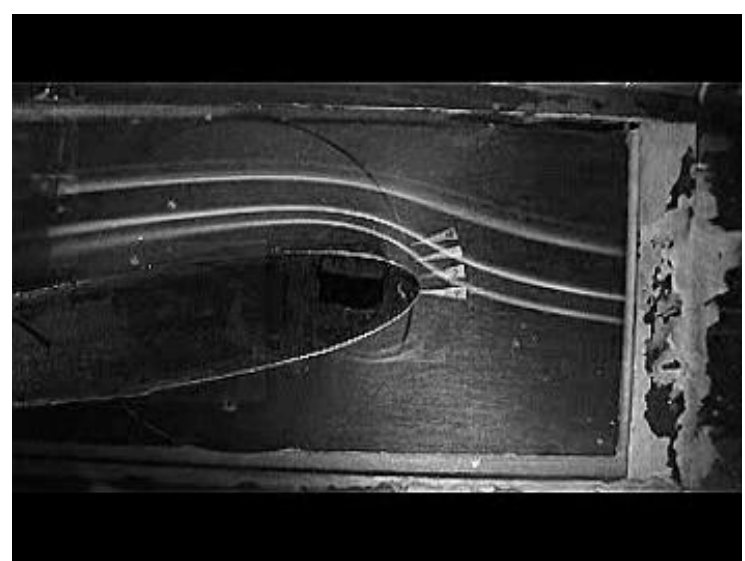

c) Airfoil at 8 degrees angle of attack attack with separation bubble

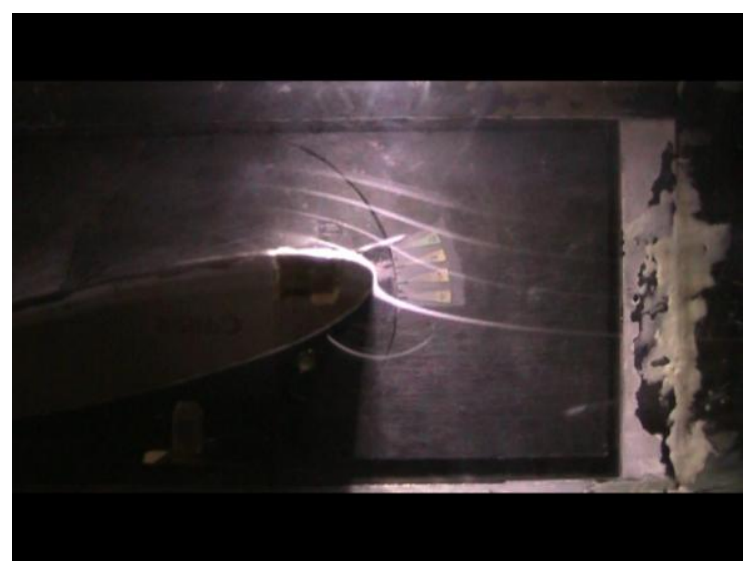

d) Airfoil at 12 degrees angle of with separation and stall

Figure 7.2: Flow visualization of baseline airfoil showing attached flow, separation bubble, and leading edge stall at $\operatorname{Re}$ of 100,000

\subsection{Short Leading Edge Separation Bubble}

The second phase of this experiment was to evaluate the effects of dynamic roughness on a short separation bubble and compare to computational results. Figure 7.3 displays the airfoil at 9.5 degrees angle of attack. To the left in the figure is the experimental flow visualization and to the right is a close-up of the CFD analysis near the leading edge region. Before the dynamic roughness is actuated, the airfoil behaves as a clean airfoil exhibiting a classic short separation bubble. Figure 7.3 shows a short separation bubble, separating about the $2 \%$ chord location and reattaching at approximately the $25 \%$ chord location. Both the experiment and the numerical analysis showed the same separation point. However, the numerically predicted reattachment point may be in error due to applying a laminar solver. In reality the flow is known to transition in the bubble. Also, in this figure the dynamic roughness apparatus mounted in the airfoil leading edge can be observed. 
As the roughness was actuated, effective flow control began to take place. In figure 7.4 the dynamic roughness frequency is $30 \mathrm{~Hz}$. This was determined by measuring the revolutions per minute of the motor which was used to drive the piston-cylinder device which in turn actuated the dynamic roughness. At $30 \mathrm{~Hz}$, some flow control began to take place. Although the short separation bubble is still present, the size of the bubble has decreased. In figure 7.5 the frequency has been increased to $60 \mathrm{~Hz}$ and the dynamic roughness flow control mechanism has eliminated the separation bubble. As the frequency was increased further the flow remained attached.

This finding is consistent with the numerical analysis and demonstrates that there is a threshold for the dynamic roughness frequency. When this frequency is reached full flow control of separation is achieved; further increases in frequency maintain the attached flow. Of course there are likely to be limits on the maximum usable frequency for dynamic roughness. As the frequency continues to increase, eventually, the normal velocity of the roughness element will approach the compressible flow regime and it is not known what affect this would have on the flow control mechanism. The other limiting frequency factor is the actuation mechanism itself. Clearly it would be beneficial to have the ability to obtain flow control at the lowest possible frequency. The maximum frequency obtainable with this particular apparatus was $160 \mathrm{~Hz}$. Theoretical studies have been done indicating effective flow control for frequencies ranging up to several thousand $\mathrm{Hz}$. 

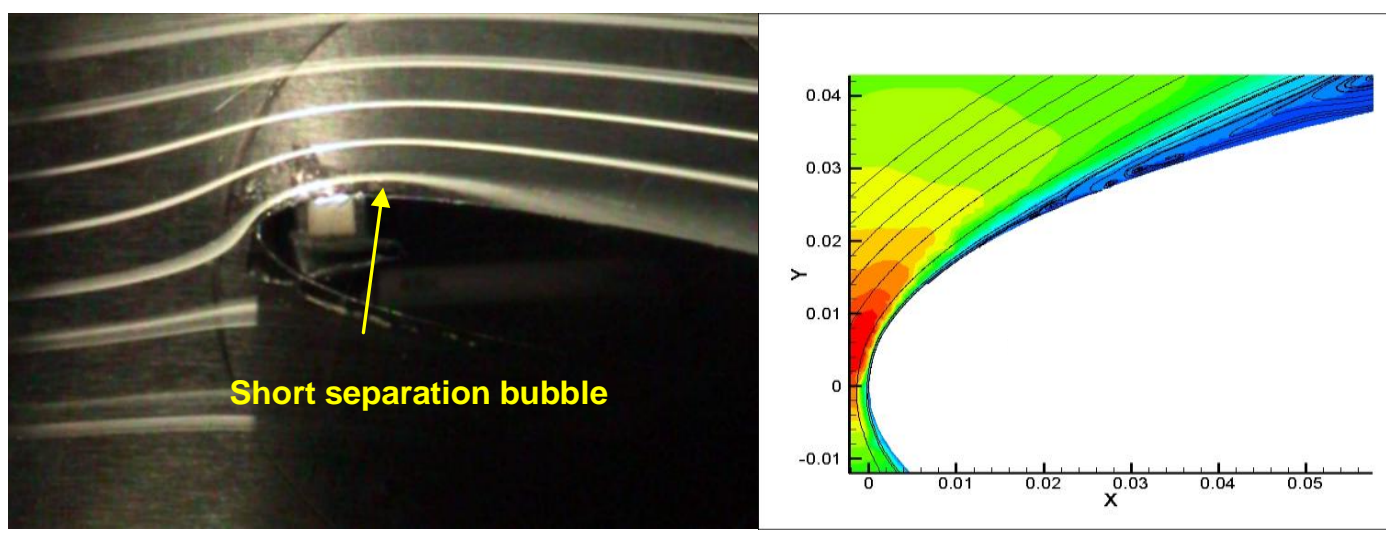

Figure 7.3: Separation bubble at 9.5 degrees angle of attack without dynamic roughness actuation
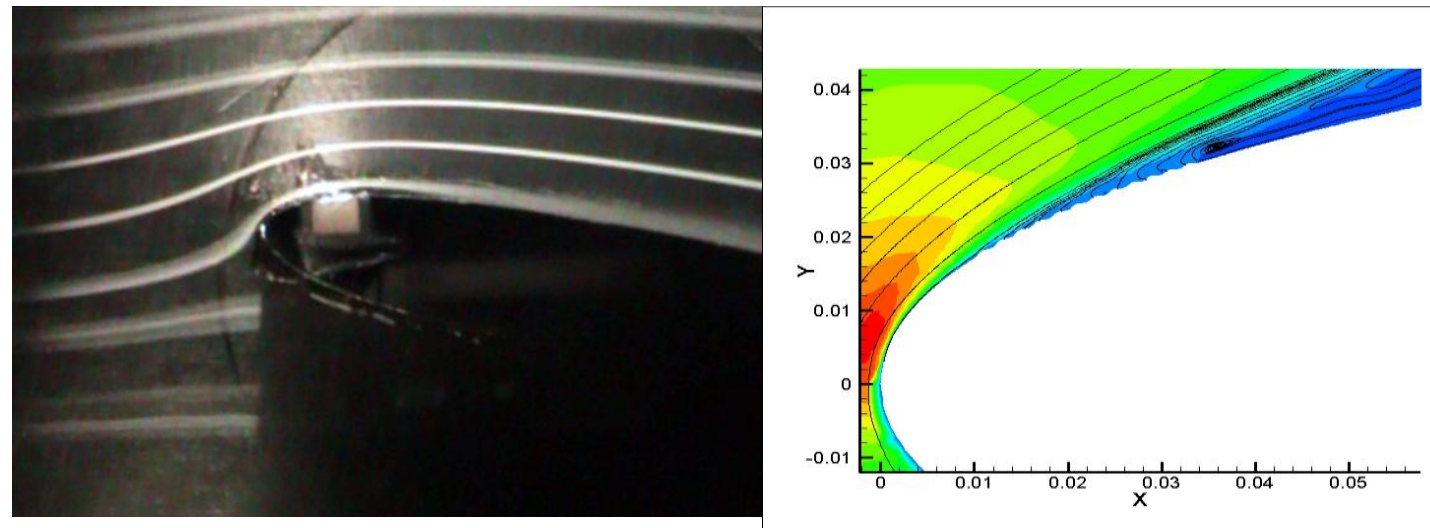

Figure 7.4: Separation bubble at 9.5 degrees angle of attack and $30 \mathrm{~Hz}$ dynamic roughness frequency actuation
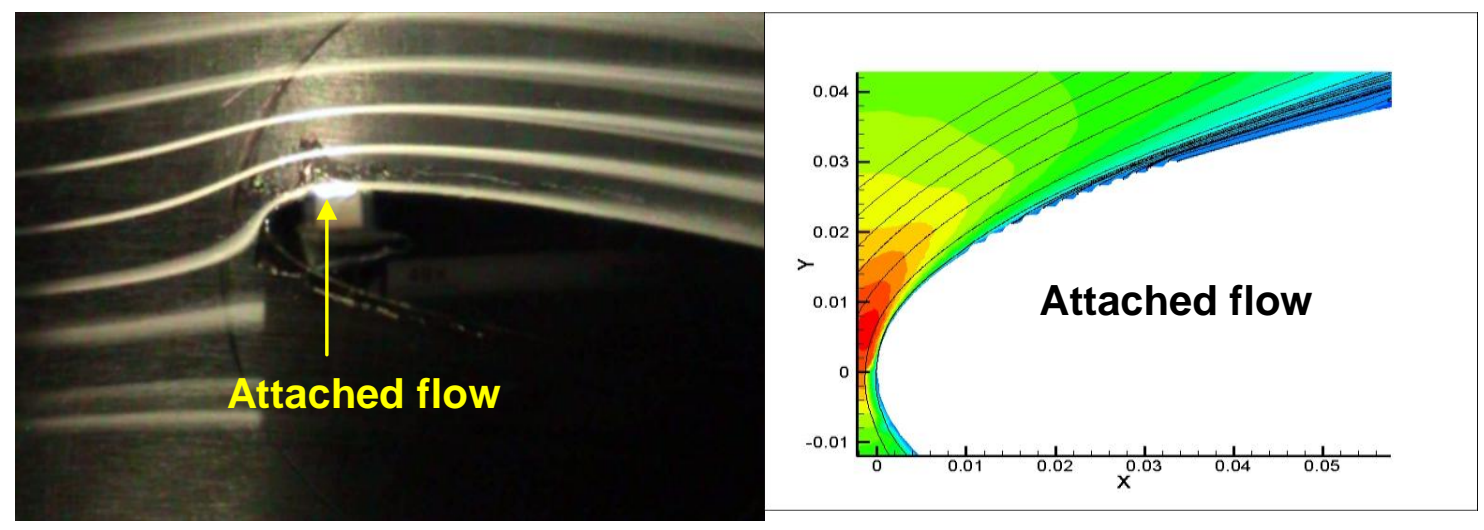

Figure 7.5: Separation bubble eliminated and attached flow achieved at 9.5 degrees angle of attack and $60 \mathrm{~Hz}$ dynamic roughness frequency actuation 
It is of significance to examine the pressure distribution on the upper surface of the airfoil. Figure 7.6 is a plot of the pressure distribution on this particular airfoil. The solid line represents data taken from Rinoi and Takemura [2000] at 9.5 degrees angle of attack and a slightly higher Reynolds number of 130,000. Also plotted in the figure are the actual pressure measurements taken in this experiment at a Reynolds number of 100,000. The second set of data points, shown by the green symbols, represents the clean airfoil case where five static pressure ports were located. The third set of data points, shown as red symbols, represents the two pressure measurements taken with the dynamic roughness apparatus in place. These two pressure ports are located just downstream of the dynamic roughness. Many more pressure orifices would have been desirable, but due to the challenge of adding the ports after the model was fabricated, it was only possible to install two.

The smokelines are of particular interest when examing the experimental data. They provided valuable information concerning the effectiveness of the dynamic roughness. They also provided additional information concerning the flow about the entire airfoil. At 9.5 degrees angle of attack and a Reynolds number of 100,000 , prior to actuation of the dynamic roughness, the smokelines exhibit a steady flow outside the bubble. When the dynamic roughness was actuated, the smokelines appeared to oscillate and "wiggle" over the surface of the airfoil downstream of the maximum thickness location. This was first thought to be a result of the dynamic roughness apparatus: However, after further examination, this was ruled out because the dynamic roughness apparatus was connected to the pressure source by a flexible tubing which cannot transmit vibrations. A second, 
more plausible explanation is that the airfoil, being mounted in a cantilever fashion, may be subject to a vibration due to the altered pressure distribution. The vibration of the airfoil, in turn, leads to a "wiggle" of the smokelines. In later tests, this issue did not exist. Also, in the 12 degree angle of attack case, it did not exist.

Typically, once a short bubble forms, there is only a small variation in surface pressure in the vicinity of the bubble. The presence of the short bubble is usually signaled by a slight plateau in the pressure curve. Once the dynamic roughness is actuated and the boundary layer becomes attached, it is not surprising to see only a very slight change in the pressure. It is the intent of this figure 7.9 to show that although the dynamic roughness suppresses the separation bubble, it does not significantly alter the pressure distribution. 


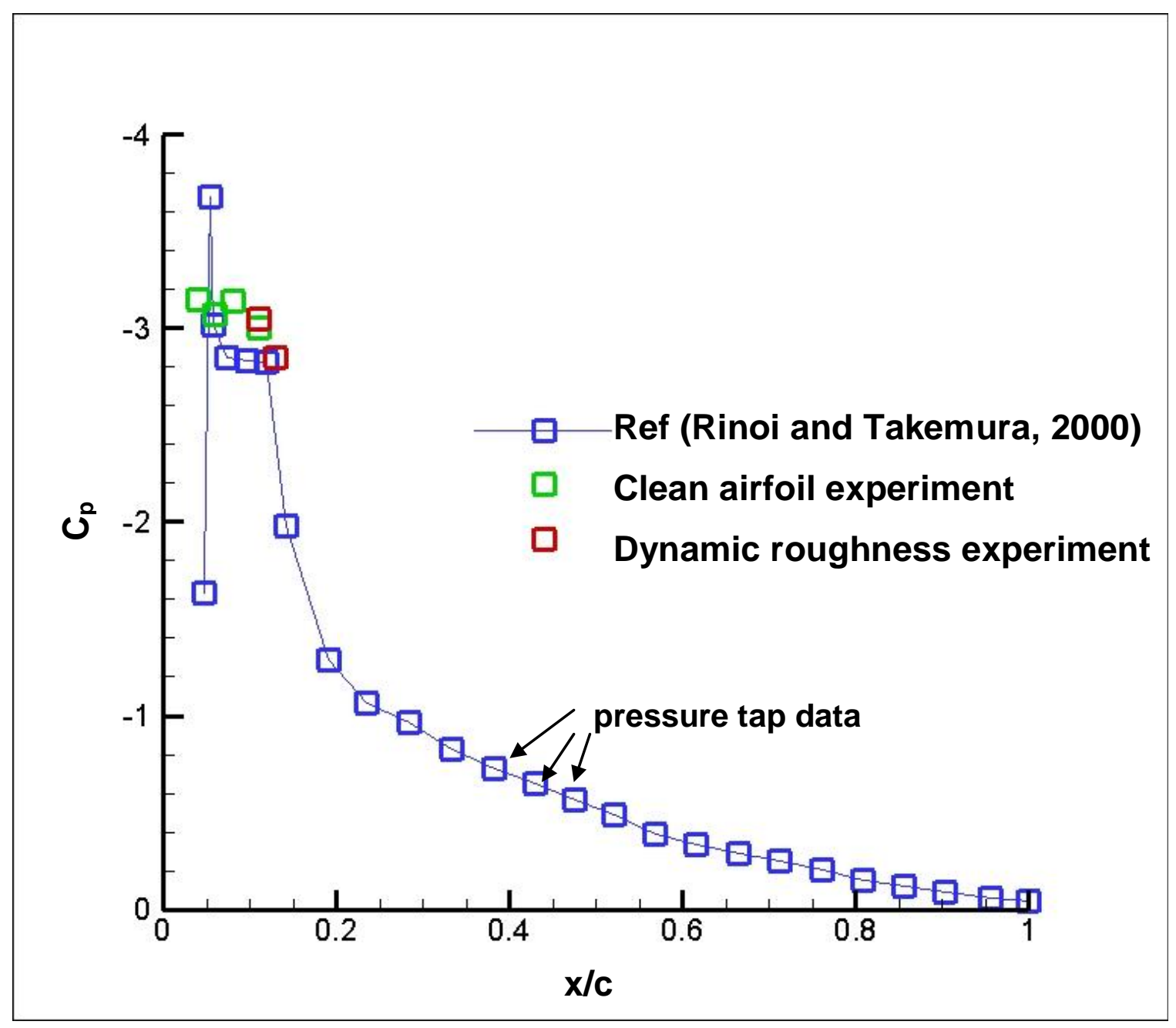

Figure 7.6: Pressure distributions for clean and dynamic roughness airfoil at 9.5 degrees angle of attack and a Reynolds number of 100,000 (experiment) and 130,000 [Rinoi and Takemura, 2000]

It is important to note that the first roughness element is at about the $3.0 \%$ chord location and the aft roughness element is located at about the $10.1 \%$ chord location. Although based on previous work it was thought that the first roughness element needed to be upstream of the separation point in this experiment the location was just aft of the separation point due to model fabrication constraints. In spite of this, the dynamic roughness still appeared to provide flow control. 


\subsection{Long Leading Edge Separation Bubble}

The case of the long separation bubble presents the most desirable case for applying effective flow control. When a long separation bubble exists, the pressure distribution can be greatly altered. Generally, a long separation bubble results in a significant loss of lift at a given angle of attack, i.e. the classic leading edge stall. At 12 degrees angle of attack, a long and highly unsteady separation bubble can be observed for the NACA 0012 . Figure 7.7 shows the airfoil at 12 degrees angle of attack prior to actuation of the dynamic roughness. Again the experimental flow visualization is shown on the left and the corresponding CFD analysis on the right. The separation point is clearly observed, however it is unclear at what point the flow reattaches to the airfoil because of the highly unsteady nature. Figure 7.8 shows the state of the flow after the dynamic roughness has been actuated. The frequency for this case was $60 \mathrm{~Hz}$. The separated flow reattached itself to the surface once actuated and the dynamic roughness provided effective flow control. Also, the separation points match for the experimental and numerical cases.

It is believed that in reality like experiment, the boundary layer, after passing through the dynamic roughness field, undergoes a natural transition process which allows it to remain attached. This was verified by inducing a turbulence model downstream of the roughness and observing the flow (section 4.3). This change in pressure can also be observed in the numerical analysis. The dark blue represents a higher negative pressure coefficient. As the flow control begins to take effect, the region of suction pressure increases. As the frequency is further increased, the flow simply appears to remain attached. 

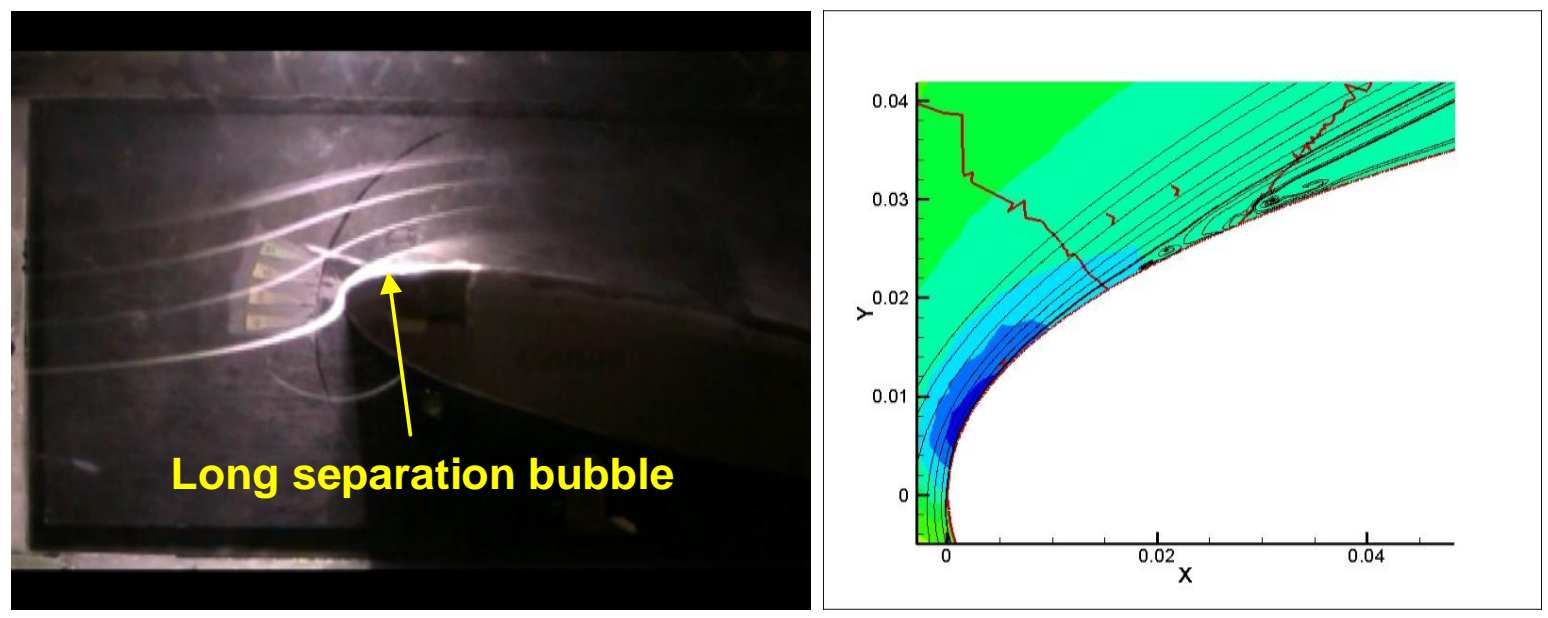

Figure 7.7: Separation bubble at 12.0 degrees angle of attack with dynamic roughness not actuated (red jagged lines bear no significance and are divisions in cell domain)
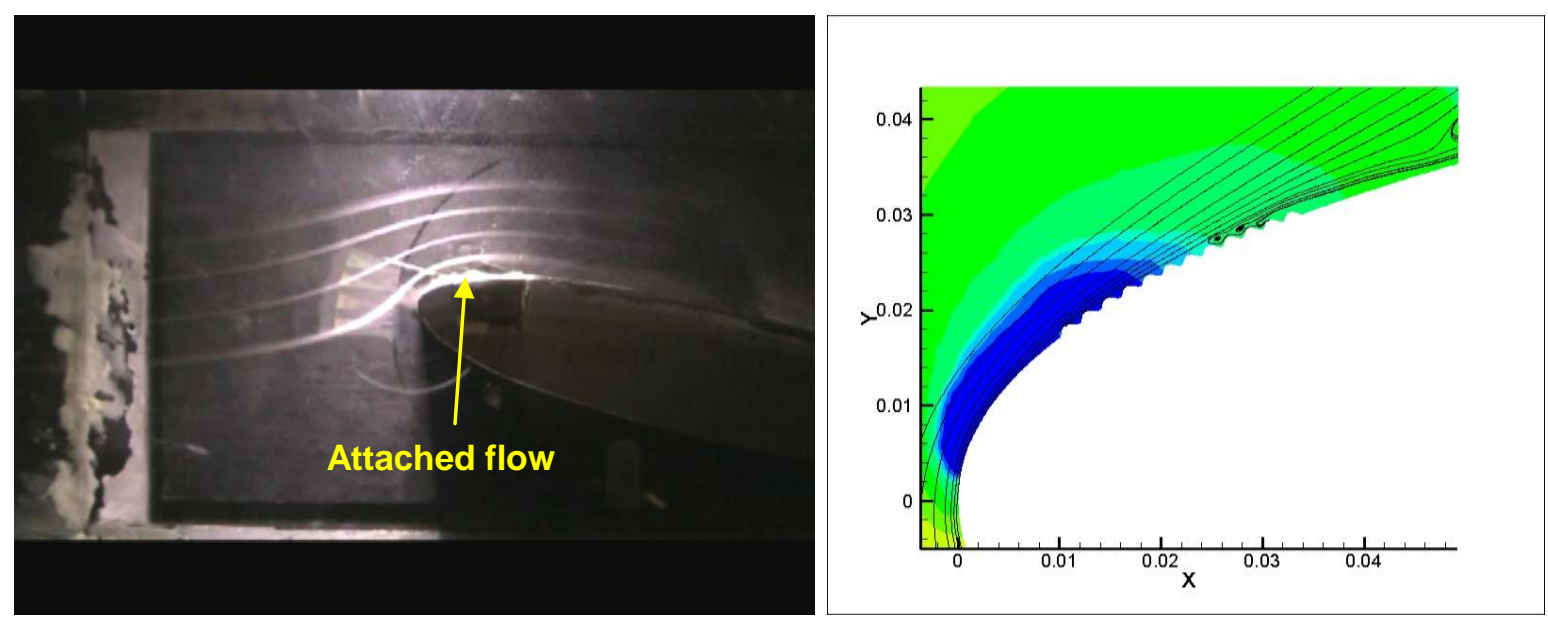

Figure 7.8: Separation bubble at 12.0 degrees angle of attack and $60 \mathrm{~Hz}$ dynamic roughness frequency

The pressure distribution case for the long bubble was significantly different than the short bubble. For the clean airfoil case, leading edge suction tends to suddenly collapse downstream of the separation point. When the dynamic roughness was actuated, the attached flow altered the pressure distribution. The suction pressure was restored and the distribution approached that of an attached flow. This, of course, would result in a significant increase in lift at a given angle of attack and an increase in leading edge 
suction (drag reduction). The intent of figure 7.10 is to show the changes in pressure when the long separation bubble is suppressed. Although the pressure measurements taken were quite limited, the intent was to successfully demonstrate a recovery of suction pressure. Figure 7.9 depicts the clean airfoil pressure distribution taken from Rinoi and Takemura [2000] as well as displaying the pressure measurements taken in this experiment for the clean airfoil and the airfoil with dynamic roughness actuated. For the clean airfoil, a pressure plateau exists downstream of the separation point. For the dynamic roughness case, the pressure was recovered once the flow control took effect although the number of pressure taps was very limited in this study. For future research, a much more detailed measurement of the upper surface pressures would be recommended. The results of this experimental and numerical study clearly show that dynamic roughness can be an effective means of flow control when leading edge separation is present. 


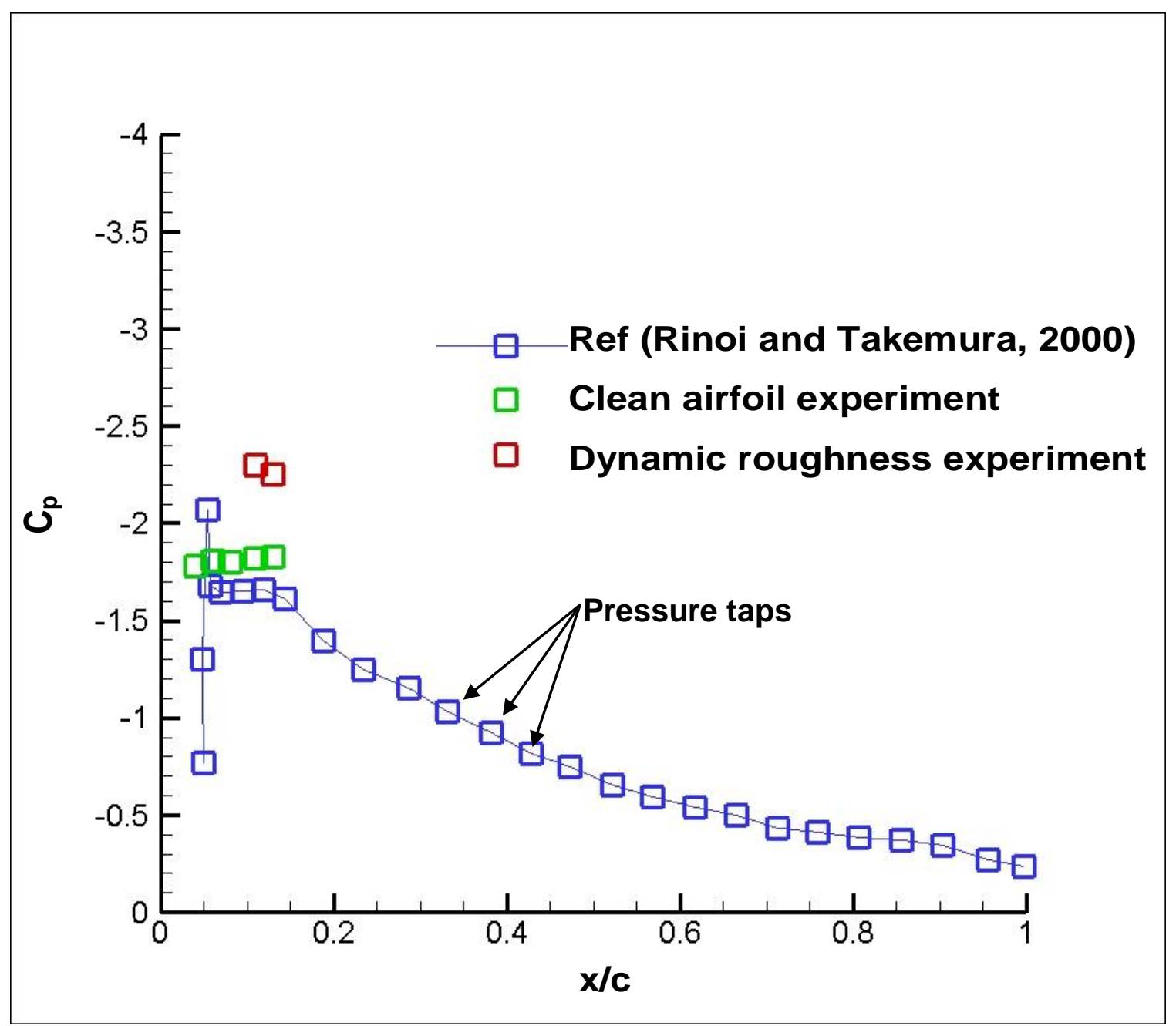

Figure 7.9: Pressure distributions for clean and dynamic roughness airfoil at 12.0 degrees angle of attack and a Reynolds number of 100,000 (experiment) and 130,000 [Rinoi and Takemura, 2000] 


\section{Chapter 8}

\section{Conclusions and Recommendations}

In this research effort, two-dimensional and three-dimensional simulations were performed in order to evaluate the mechanism of dynamic roughness as a means to provide effective leading edge flow control. In addition, wind tunnel experiments were performed to validate the concept. The model used in this study was a NACA 0012 airfoil at a Reynolds number of 100,000 and 150,000. Numerical analysis was accomplished using the commercial code Fluent ${ }^{\circ}$. The code was set up to accommodate the unsteady flow physics involved in laminar separations and moving walls (dynamic roughness).

Results of this study indicate that dynamic roughness can be used as an effective means of leading edge flow control. It has been demonstrated that dynamic roughness has the ability to eliminate both the short and long separation bubbles inherent in a low Reynolds number leading edge flow operating at a moderate angle of attack. Also, roughness amplitudes on the order of only a few percent of the boundary layer thickness can provide flow control, provided the frequency is high enough. This work confirmed the existence of a coupling between the frequency and amplitude for dynamic roughness. In addition, the work also showed there is a frequency threshold below which the dynamic roughness acts as static roughness and is ineffective for flow control. Likewise there is an amplitude threshold at a given frequency below which the dynamic roughness will act as static roughness. 
While the study of Huebsch [2006] concentrated on two-dimensional dynamic roughness elements whose sizes were comparable to the boundary layer thickness, and which could clearly disrupt the boundary layer, the current study shows that this global alteration of the boundary layer can be maintained even when the amplitude of the dynamic roughness decreases to a few percent of the boundary layer thickness, providing that the frequency is significantly increased. This result makes sense from a Reynolds stress perspective. The Reynolds stresses within the boundary layer are created by the flow velocities which are generated, in part, by the velocity of the moving roughness elements. If the amplitude of oscillation is decreased but the frequency is increased then the velocity magnitudes within the boundary layer induced by the dynamic roughness can be maintained, and the effective magnitude of the Reynolds stresses induced by the dynamic roughness can also be maintained. These ideas are currently being explored in a more rigorous setting.

Also, a potentially significant finding was that in the experimental case, the dynamic roughness field actually originated downstream of the known separation point. This was due to fabrication constraints in building the model. In spite of this, the flow visualization showed that the dynamic roughness still eliminated the separation bubble. Past work had indicated that the dynamic roughness would likely need to be at or upstream of the laminar separation point. More quantitative analysis (e.g PIV analysis) is needed to verify this finding, but it shows that perhaps dynamic roughness is more robust than previously thought and may adapt well to off-design conditions. Also, it is believed that dynamic roughness is not merely another tool used to trip the boundary layer from laminar to 
turbulent flow in order to keep it attached. This was supported by the fact that if the dynamic roughness was held at its maximum amplitude, acing like static roughness, the critical Reynolds number would be far below that required to cause transition.

The overall results of this research study can be summarized as follows.

- Dynamic roughness has to ability to eliminate both the short and long separation bubbles inherent in a low Reynolds number leading edge flow operating at moderate angles of attack. Although the elimination of the short bubble does not greatly alter the pressure distribution, the elimination of the long bubble does favorably alter the pressure distribution. This type of flow control would clearly increase aerodynamic performance. These research results were arrived at by utilizing two-dimensional and three-dimensional numerical analysis and wind tunnel experiments. This research represents the first time dynamic roughness was numerically modeled as three-dimensional geometries. Also, this is the first time that dynamic roughness was experimentally determined to be effective in controlling flow separation.

- In the experimental work, the dynamic roughness was able to eliminate the separation bubble even though it started downstream of the clean separation point. This is significant and implies that this method is more robust than previously thought and may adapt well to off design conditions. 
- The three-dimensional numerical results indicated that there was a certain frequency threshold where the flow sees dynamic roughness as static roughness and the flow control becomes ineffective.

- The CFD results also showed that as the amplitude of the roughness was decreased a higher frequency was required to meet this threshold in order to maintain flow control. This indicated that there was a coupling between the dynamic roughness frequency and amplitude. The exact coupling has not yet been determined and will likely be a function of the Reynolds number. It was determined; however, that roughness amplitude as small as a couple percent of the boundary layer thickness could be effective in providing flow control.

- Numerical results indicate that there is little difference in separation control when comparing the two different hump geometries, three-dimensional axisymmetric humps and spanwise ridges.

- The CFD results and experimental results of this study agreed fairly well.

In summary, this type of flow control may have the potential to be more efficient than traditional boundary layer control methods while gaining the desired improvements in aerodynamic efficiency. Given the results of this study, it seems justifiable to continue research in this area. Future research could include the following.

- Refinement of the numerical methods applied in studying three-dimensional parameter space. These parameter may include roughness height, location, 
frequency dependence, and geometry. For more physical insight, the CFD methodology may have to advance to DNS to gain detailed data on the state of the boundary layer and the physical mechanisms that actually allow the dynamic roughness to eliminate the separation.

- Continued experimental studies bases on the numerical results. Experimental studies could include detailed surface pressure measurements along the entire surface, continued flow visualization techniques, and detailed velocity measurements of the flow near the surface of the airfoil. Future generation experimental models need to have an increased number of pressure taps including in the dynamic roughness region. Near-surface velocity measurements will need to be gathered with more sophisticated equipment such as particle image velocimetry.

- Evaluation of mechanisms to actuate the dynamic roughness field. Some of these mechanisms include pzioelectric actuation, liquid crystal actuation, and pure mechanical actuation. 


\section{References}

Acarlar, M.S. and Smith, C.R., "A Study of Hairpin Vortices in a Laminar Boundary Layer," Journal of Fluid Mechanics, Vol. 175, pp 1-41, 1987.

Alam, M. and Sandham, N.D., "Direct Simulation of Short Laminar Separation Bubbles With Turbulent Reattachment," Journal of Fluid Mechanics ,No. 403, pp 223-250, 2000.

Abbott, I.H. and Von Doenhoff, A.E., "Theory of Wing Sections," Dover Publications, New York, 1959.

Arena, A.V. and Mueller, T.J., "Laminar Separation, Transition, and Turbulent Reattachment Near the Leading Edge of Airfoils," AIAA Journal, Vol. 18, No. 7, pp 747$757,1980$.

Attinello, J.S., “ Design and Engineering Features of Flap Blowing Installations; Boundary Layer and Flow Control, Principles and Applications," Vol. 1, New York, Pergamon Press, 1961.

Bragg, M.B., Kerho, M.F. and Cummings, S.L., "Airfoil Boundary Layer Due to Leading Edge Roughness," AIAA paper 95-0536, presented at the $33^{\text {rd }}$ Aerospace Sciences Meeting and Exhibit, 1995.

Baragona, M., Bijl, H. and Van Torren, M., "Bubble Bursting and Laminar Separation Unsteadiness on a Multi Element High Lift Configuration," Flow Turbulence and Combustion, No 71, pp 279-296, 2003.

Braslow, A.L., Hicks, R.M. and Harris, R.V., "Use of Grit-type Boundary Layer Transition Trips on Wind Tunnel Models," NASA TN D-3579, Sept 1966.

Brzek, B.G. and Cal, R.B., "Transitionally Rough Zero Pressure Gradient Turbulent Boundary Layers,” Experimental Fluids, Vol. 44, pp 115-125, 2008.

Bushnell, D.M. and Hefner, J.N., ” Effect of Compliant Wall Motion on Turbulent Boundary Layers," Physics of Fluids, Vol. 20, No. 10, pp S31-S48, 1977.

Buchingham, A.C. and Chun, R.C., "Compliant Material Coating to a Turbulent Boundary Layer," QIQQ-82-1027, AIAA/ASME Joint Thermo physics, Fluids, Plasma and Heat Transfer Conference, Jun 7-11, St. Louis, Missouri, 1982.

Brumby, R.E., "Wing Surface Roughness, Cause and Effect, "DC Flight Approach, Jan, 1979. 
Carpender, P.W., Lucey, A.D., Davies, C., "Progress on the Use of Compliant Walls For Laminar Flow Control,” Journal of Aircraft, Vol. 38, No. 3, May-June 2001.

Choudhuri, P.G. and Knight, D.D., "Two Dimensional Leading Edge Separation on a Pitching Airfoil,” AIAA Journal, Vol. 32, No. 4, pp 673-681, 1994.

Deng, S., Jiang, L. and Liu, C., "DNS for Flow Separation Around an Airfoil by Steady and Pulsed Jets," NATO RTO-MP-AVT-111 presented at the meeting on Enhancement of NATO Military Flight Vehicle Performance by Management of Interacting Boundary Layer Transition and Separation, Prague, Czech Republic, October 2004.

Desalvo, M.E. and Glezer, A. "Airfoil Aerodynamic Performance Modification Using Hybrid Surface Actuators," AIAA 2005-0872.

Dryden, H. L. and Klueth, A. M. "Effect of Turbulence in a Wind Tunnel," NACA Technical Report 348, 1929.

Fasel, H., "Investigation of the Stability of Boundary Layers by a First Order Model of the Navier-Stokes Equations," Journal of Fluid Mechanics, Vol. 78, pp 355-383, 1796.

Fluent Inc., "Fluent User's Guide, Fluent, 2009. www.fluentusers.com

Floryan, J.M. and Dallman, U., "Flow Over a Leading Edge With Distributed Roughness," Journal of Fluid Mechanics, Vol. 216, pp 629-656, 1990.

Gaster, M., "The Structure and Behavior of the Laminar Separation Bubbles," AGARD CP-4, pp 819-854, 1966.

Greenblatt, D. and Wygnanski, I.J., "The Control of Flow Separation by Periodic Excitation,” Progress in Aerospace Sciences, pp 487-545, Elsevier Pergamon, 2000.

Gregory, N. and Walker, W.S., "The Effects of Transition of Isolated Surface Excrescences in the Boundary Layer,” British ARC, Res. Memo 2779, 1956.

Gregory, N. and O'Reilly, C.L., 'Low Speed Aerodynamic Characteristics of NACA 0012 Aerofoil Section, including the Effects of Upper Surface Roughness Simulating Hoar Frost," Aeronautical Research Council, Royal Aircraft Establishment, RM No 3726, 1973.

Gregory, N. and Wilby, P.G., "NPL 9615 and NACA 0012; A Comparison of Aerodynamic Data," Aeronautical Research Council, Royal Aircraft Establishment, CP No 1261, 1973. 
Hunsaker, R. and Huebsch, W.W., "Parametric Study of Surface Roughness as a Mechanism for Flow Control," AIAA-2005-4732, 23 ${ }^{\text {rd }}$ Applied Aerodynamics Conference, Toronto, Ontario, June 6-9, 2005.

Hortin, H.P., "A Semi-empirical Theory For the Growth and Bursting of Laminar Separation Bubbles,” Aeronautical Research Council, CP 1073, 1969.

Huebsch, W.W., "Dynamic Surface Roughness for Aerodynamic Flow Control," AIAA2004-587, AIAA Aerospace Sciences Meeting and Exhibit, Jan 5-8, Reno, Nevada, 2004.

Huebsch, W.W. , "Two Dimensional Simulation of Dynamic Surface Roughness for Aerodynamic Flow Control," Journal of Aircraft, Vol. 43, No. 2, pp 353-362, 2006.

Huebsch, W.W. and Rothmayer, A.P., “ Small Scale Roughness Effects on Laminar Separation," Theoretical and Computational Fluid Dynamics, Vol. 17, No. 2, Dec 2003.

Huebsch, W.W. and Rothmayer, A.P., "Numerical Prediction of Unsteady Vortex Shedding for Large Leading Edge Surface Roughness," Computers and Fluids, Vol. 33, No. 3, pp 405-434, 2004.

Jacobs, E. and Sherman, A., "Airfoil Section Characteristics as Affected by the Changes in Reynolds Number," NACA TR-586, 1937.

Kerho, M.F., "Effect of Large Distributed Roughness Near an Airfoil Leading Edge Boundary Layer Development and Transition," $\mathrm{PhD}$ Dissertation, University of Illinois Urbanna-Champaign, Urbanna, IL, 1995.

Kerho, M.F. and Bragg, M.B., “Airfoil Boundary Layer Development and Transition With Large Leading Edge Roughness,” AIAA Journal, Vol. 35, No. 1, pp 75-84, 1997.

Klebanoff, P.S. and Tidstrom, K.D., "Mechanism by Which Two-dimensional Roughness Elements Induce Boundary Layer Transition," Physics of Fluids, Vol. 15, pp 1173-1188, 1972.

Kotapati-Apparo, R. and Squirs, K., " Prediction of the Flow over an Airfoil at Maximum Lift," AIAA 2004-0259, Aerospace Sciences Meeting, Reno, Nevada, Jan 5-8, 2004.

Kramer.M.O., "Boundary Layer Stabilization by distributed Damping," Journal of Aeronautical Sciences, Vol. 24, No. 6, 1957.

Liebeck, R.H., "A Class of Airfoils Designed for High Lift in Incompressible Flow," Journal of Aircraft, Vol. 10, October 1973.

Lin, J.C. and Pauly, L.L., "Low Reynolds Number Separation on an Airfoil," AIAA Journal 348, 1996. 
Matheis, B.D., Huebsch, W.W., and Rothmayer, A.P., "Separation and Unsteady Vortex Shedding From Leading Edge Surface Roughness," NATA-OTAN , RTO-MP-IST-999, 2004.

McCullough, G.B., and Gault, D.E., "Examples of Representative Types of Airfoil Section Stall at Low Speed," NACA Technical Note 2502, 1955.

Morin, B.L. and Patrick, W.P., "Detailed Studies of a Large Scale Laminar Separation Bubble on a Flat Plate," United Technologies Research Center Report no. R 910956786-1, 1991.

Morkovin, M. V., "Critical Evaluation of Transition From Laminar to Turbulent Shear Layers with Emphasis on Hypersonically Traveling Bodies," AFFDL TR-68-149, 1969.

Mueller, T.J. and Batill, S.M., "Experimental Studies of Separation on a Two Dimensional Airfoil at Low Reynolds Numbers," AIAA Journal, Vol. 20, pp 457-463, April 1982.

Nakae,Y., Motohashi, T., Komurasaki, S. and Kuwahera, K., "The Role of Separation Bubble on an Airfoil at Low Reynolds Numbers," Computational Fluid Dynamics 2006: Proceedings from the Fourth International Conference on Computational Fluid Dynamics, ICCFD4, Ghent, Belgium, July, 2006.

Orlov, D.M., Apker, T., He, C., Othman, H. and Corke, T.C., " Modeling and Experiment of Leading Edge Separation Control Using SDBD Plasma Actuators," AIAA 2007-0877, Aerospace Sciences Meeting, Jan 5-8, Reno, Nevada, 2007.

Pauley, L.L., Moin, L.L. and Reynolds, W.C., "The Structure of Two Dimensional Separation," Journal of Fluid Mechanics, No. 220, 1990.

Peterson, J.B. and Horton, E.A., "An Investigation of the effect of a Highly Favorable Pressure Gradient on Boundary Layer Transition as Caused by Various Types of Roughness on a 10 foot Diameter Sphere at Subsonic Speeds," NASA Memo 2-8-59-L, April, 1959.

Rediniotis, O., Lagoudas, D., Mani, R., Traub, L., Allen, R., and Karniadakis, G., "Computational and Experimental Studies of an Active Skin for Turbulent Drag Reduction," AIAA-2002-2380, $1^{\text {st }}$ Flow Control Conference, St. Louis, MO, July, 2002.

Rinoi, K., "Measurements of a Short Bubble and Long Bubble Formed on a NACA 63009 Airfoil," Journal of Aeronautical and Space Sciences Japan 38, pp 249-257, 1990.

Rinoie, K. and Takemura, N., "Oscillating Behavior of Laminar Separation Bubbles Formed on an Aerofoil Near Stall," The Aeronautical Journal, pp 153-163, March, 2004. 
Ripley, M.D. and Pauley, L.L., "The Unsteady Nature of Two Dimensional Steady Laminar Separation," Physics of Fluids, Vol. 5, No 12, 1993.

Rothmayer, A.P. and Huebsch, W.W., "On the Modification of Laminar Boundary Layers Using Unsteady Surface Actuation,” To be submitted, May, 2010.

Rusak, Z. and Morris, W.J., "On the Prediction of Stall Onset at Moderately High Reynolds Number Flows,” AIAA 2005-0086, 1986.

Roberts, W.B., "Calculation of Laminar Separation Bubbles and Their Effect on Airfoil Performance,” AIAA Journal, Vol. 18, No. 1, pp 25-36, 1980.

Saric, W.S., Reed, H.L. and White, E.B. "Stability and Transition of Three-dimensional Boundary Layers," Annual Review of Fluid Mechanics, Vol. 35, pp 413-440, 2003.

Schlichting, H. and Gersten, K., Boundary Layer Theory, $8^{\text {th }}$ Revised and Enlarged Edition, Springer-Verlag, Berlin, Heidelburg, 2000.

Sinha, S.K., "Flow Separation With Microflexural Wall Vibrations," Journal of Aircraft, Vol. 38, No. 3, pp 496-504, 2001.

Spalart, P.R. and Strelets, M.K., "Mechanisms of Transition and Heat Transfer in a Separation Bubble,” Journal of Fluid Mechanics, Vol. 403, pp 329-349, 2000.

Tani, I., " Low Speed Flows Involving Separation Bubbles,” Aeronautical Research Institute, University of Tokyo.

Stratford, B.S., "An Experimental Flow with Zero Skin Friction Throughout the Region of Pressure Rise," Journal of Fluid Mechanics, Vol. 5, 1959.

Tani, I., "Effect of Two-dimensional Roughness on Laminar Flow," Boundary Layer and Flow Control, edited by G.V. Lachmann, Vol. 2, Pergamon, Oxford, England, UK, pp 637-656, 1961.

Tani, I., "Boundary Layer Transition," Annual Review of Fluid Mechanics, Vol. 1, pp169-196, 1969.

White, E.B., "Experiments in Transient Growth and Roughness Induced Bypass Transition," Case Western Reserve, Mechanical and Aerospace Engineering, F49620-02$1-0058$.

Wygnanski, I. and Darabi, A. , "Active Management of Naturally Separated Flow Over a Solid Surface; The Forced Reattachment Process," Journal of Fluid Mechanics, Vol. 510, pp 105-129, 2004. 
Vatsa, V.N. and Carter, J.E., "Analysis of Airfoil Leading Edge Separation Bubbles," AIAA Journal, Vol. 22, No. 12, pp 1967-1703, 1984.

Valarezo, W.O., Lynch, F.T. and McGhee, R.J., "Aerodynamic Performance Effects Due to Small Leading Edge Ice (roughness) on Wings and Tails," Journal of Aircraft, Vol. 30, No. 6, Nov-Dec 1993. 


\section{Appendix A}

\section{User Defined Function Algorithm}




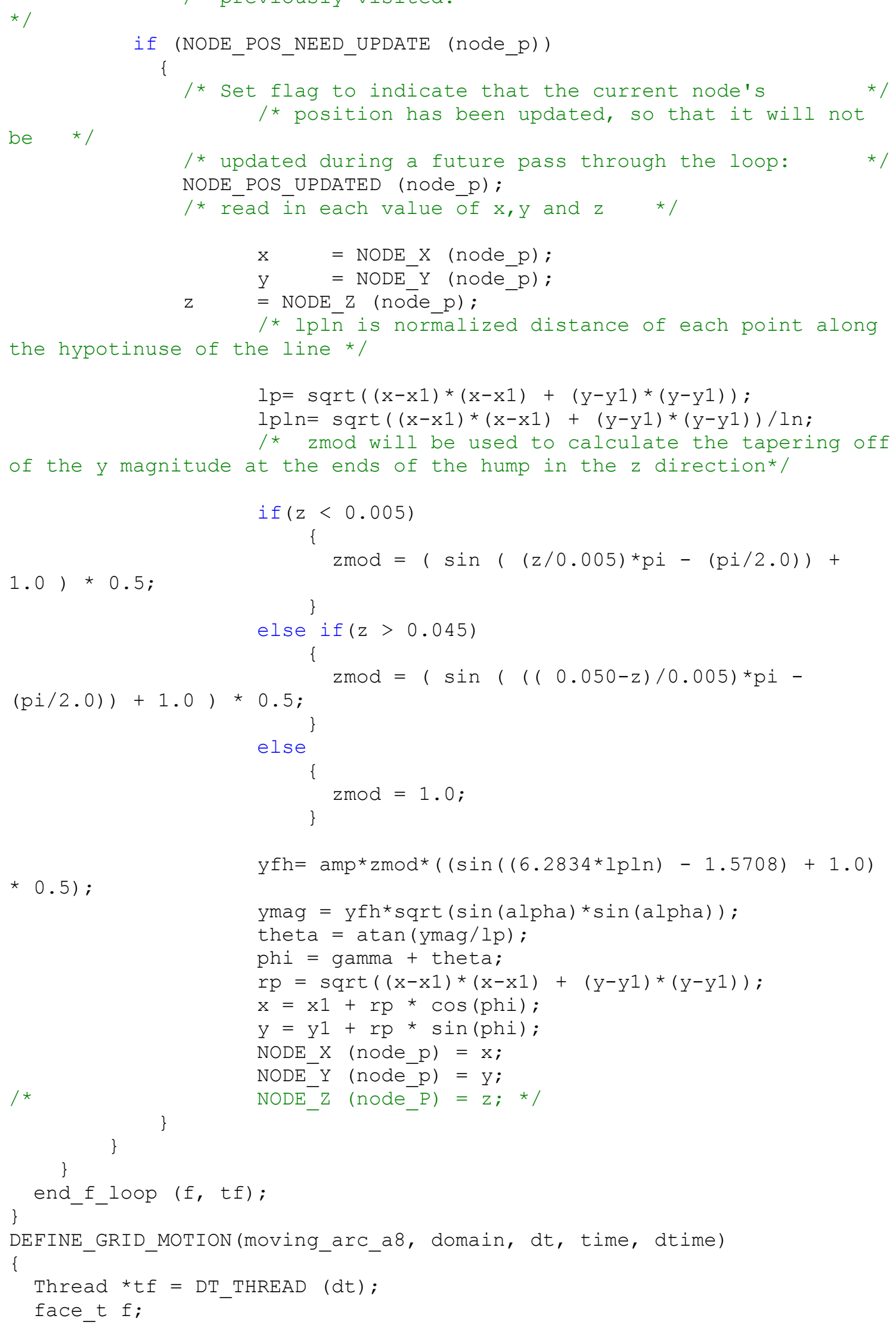




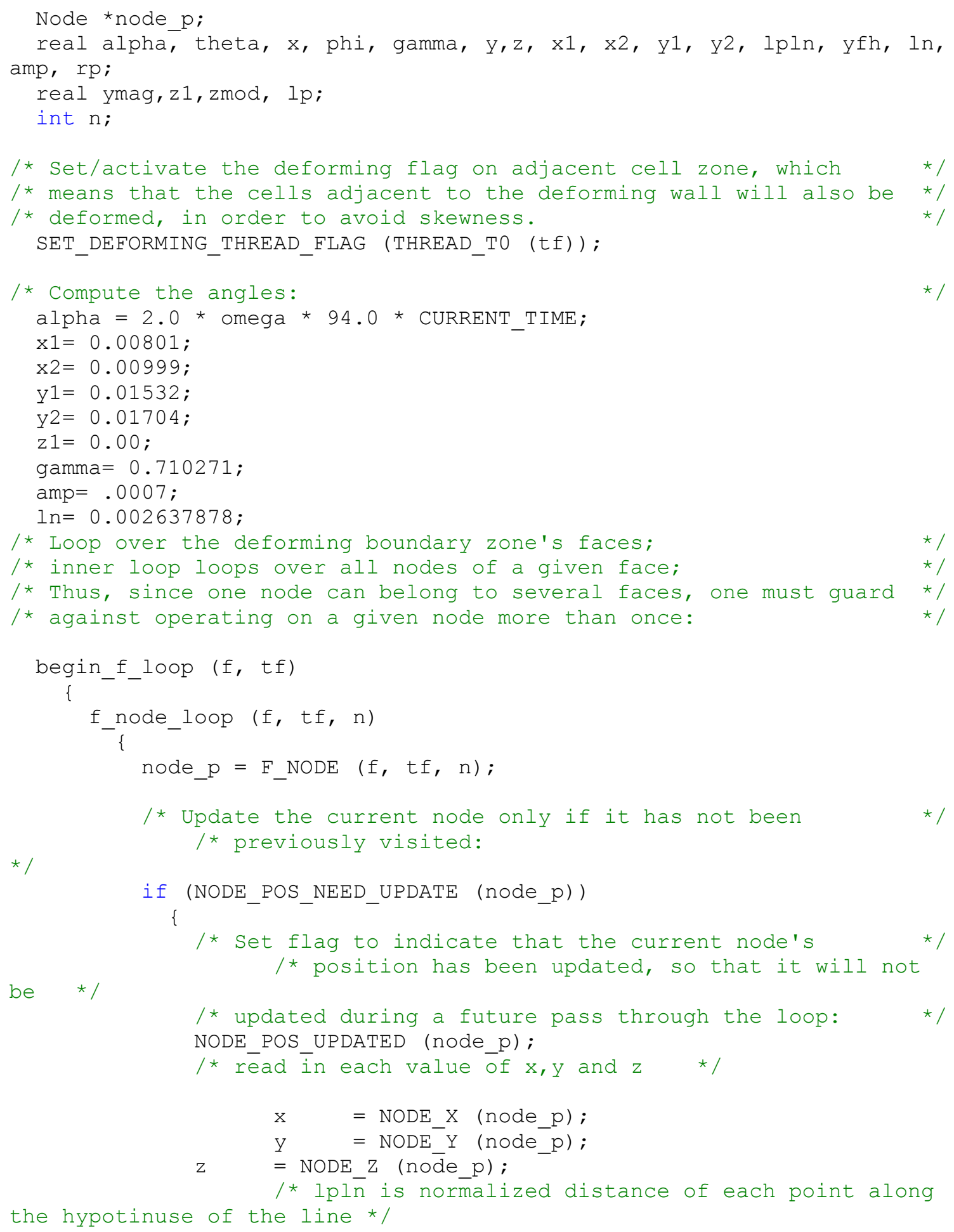




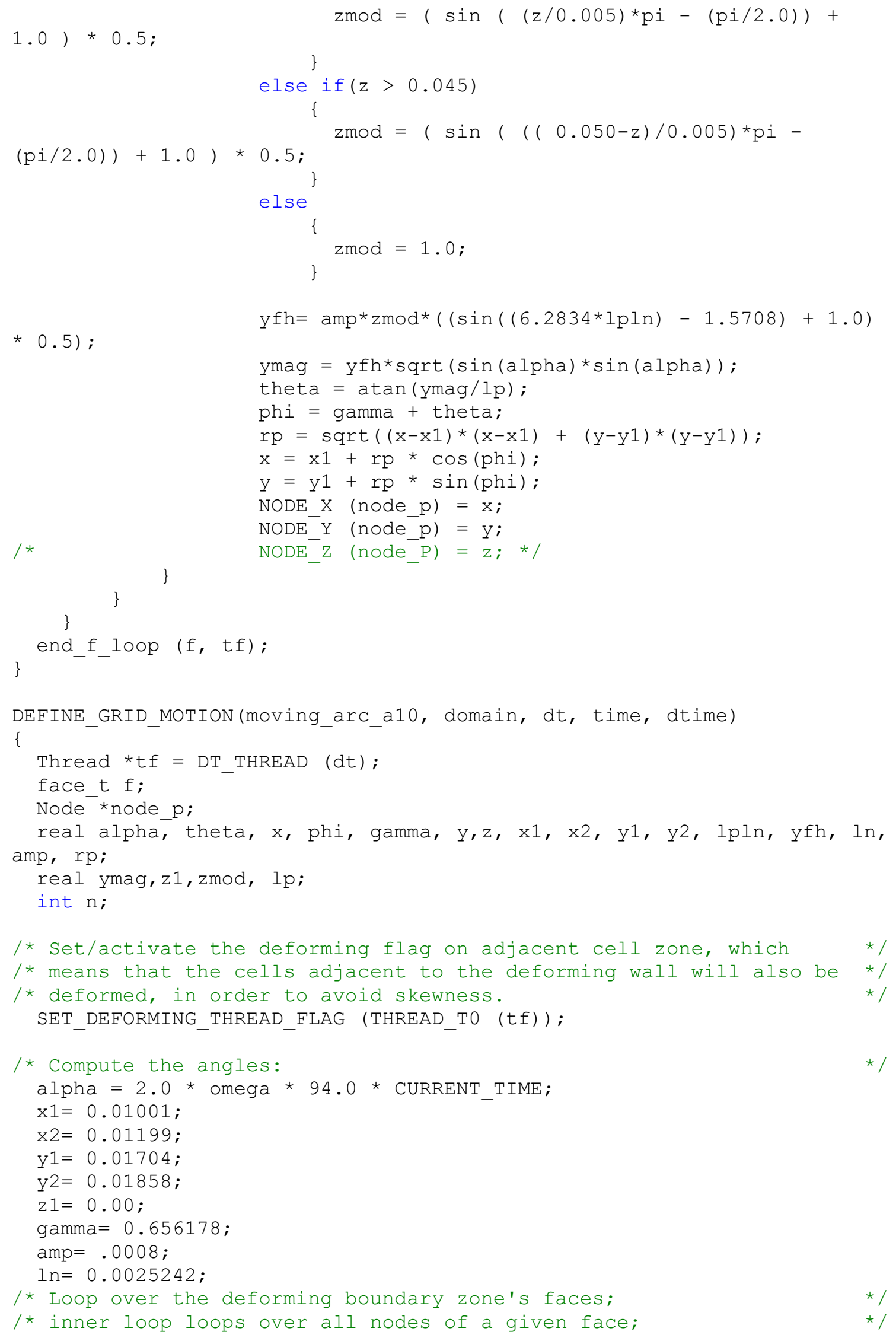




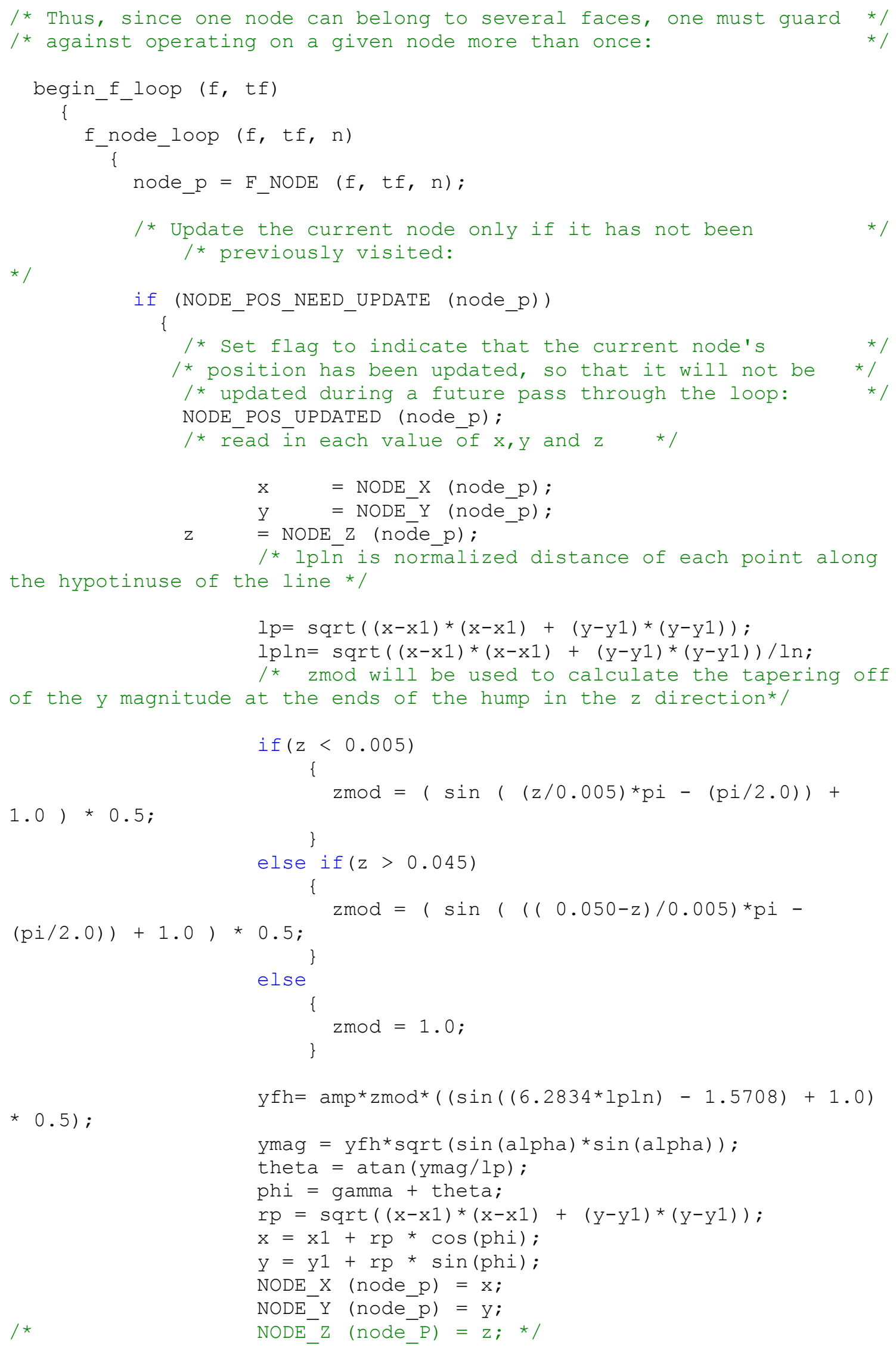




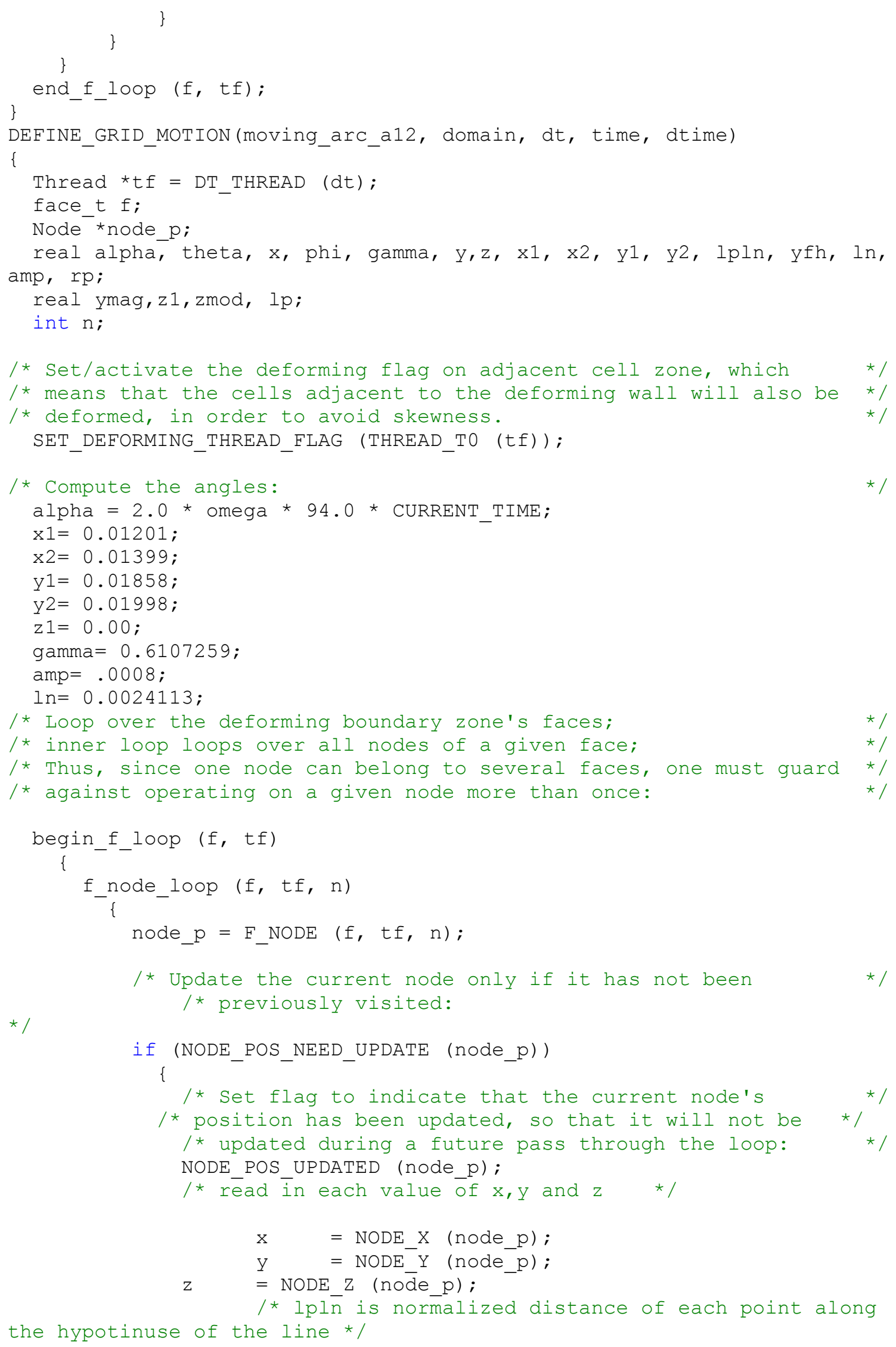




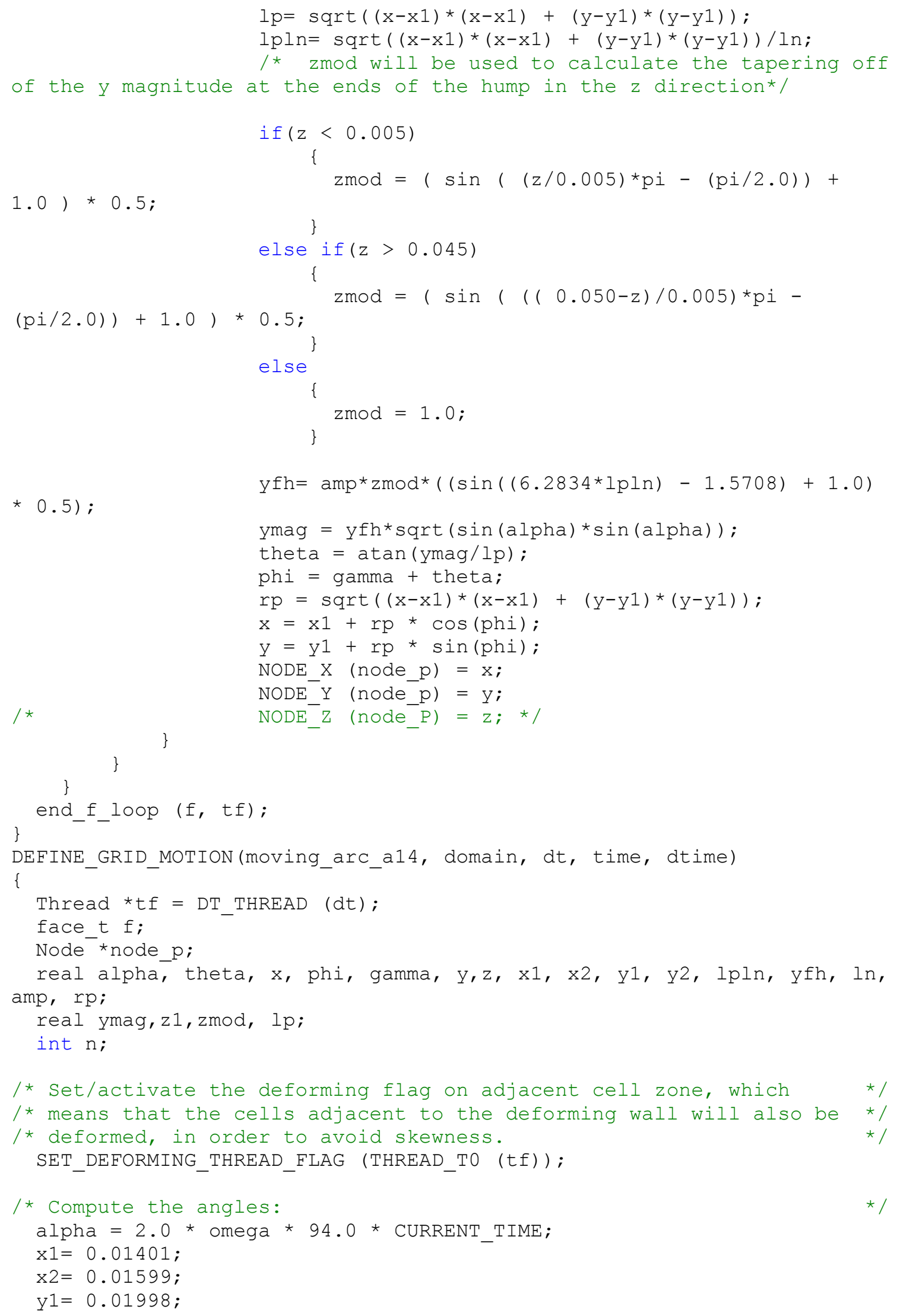




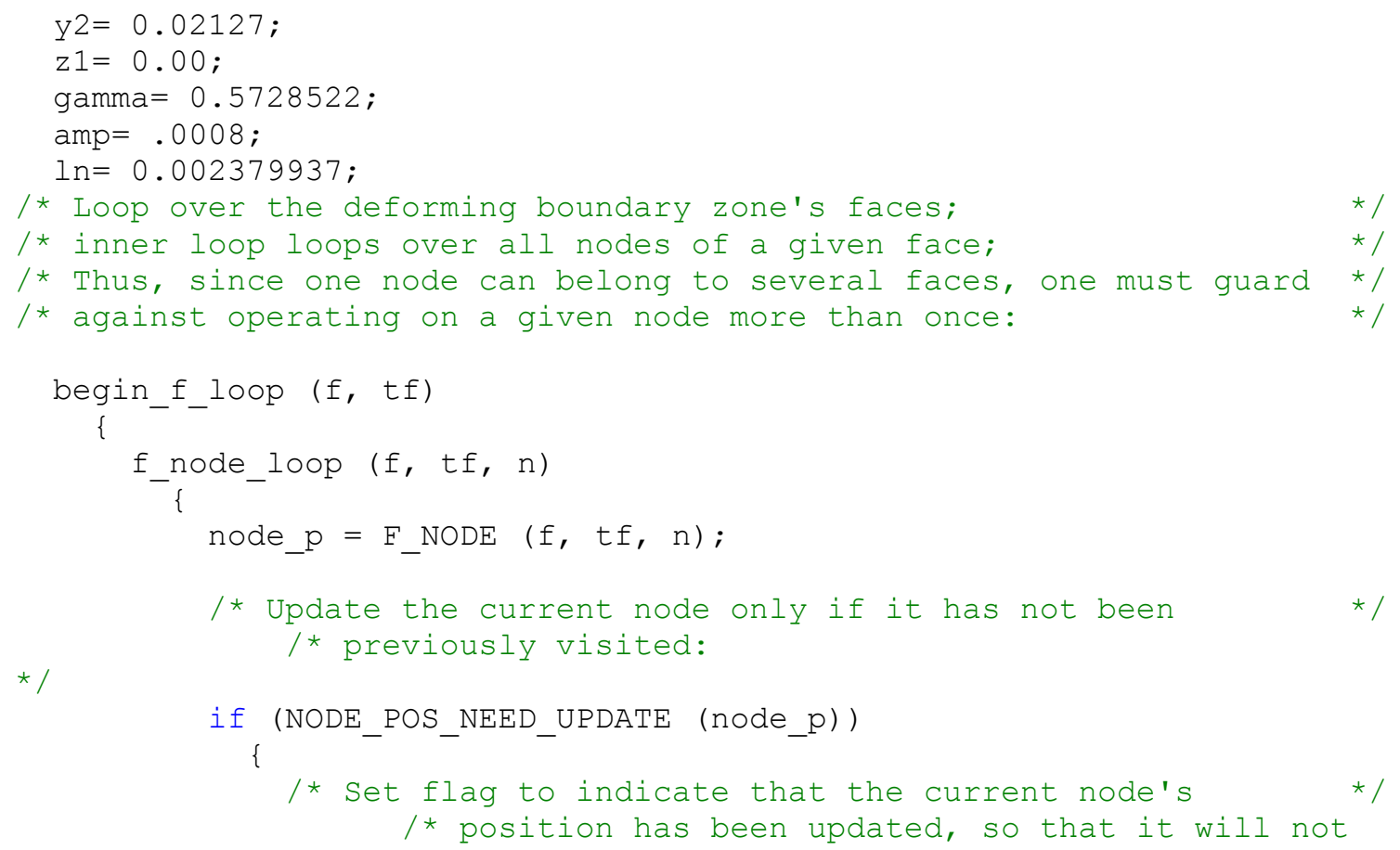




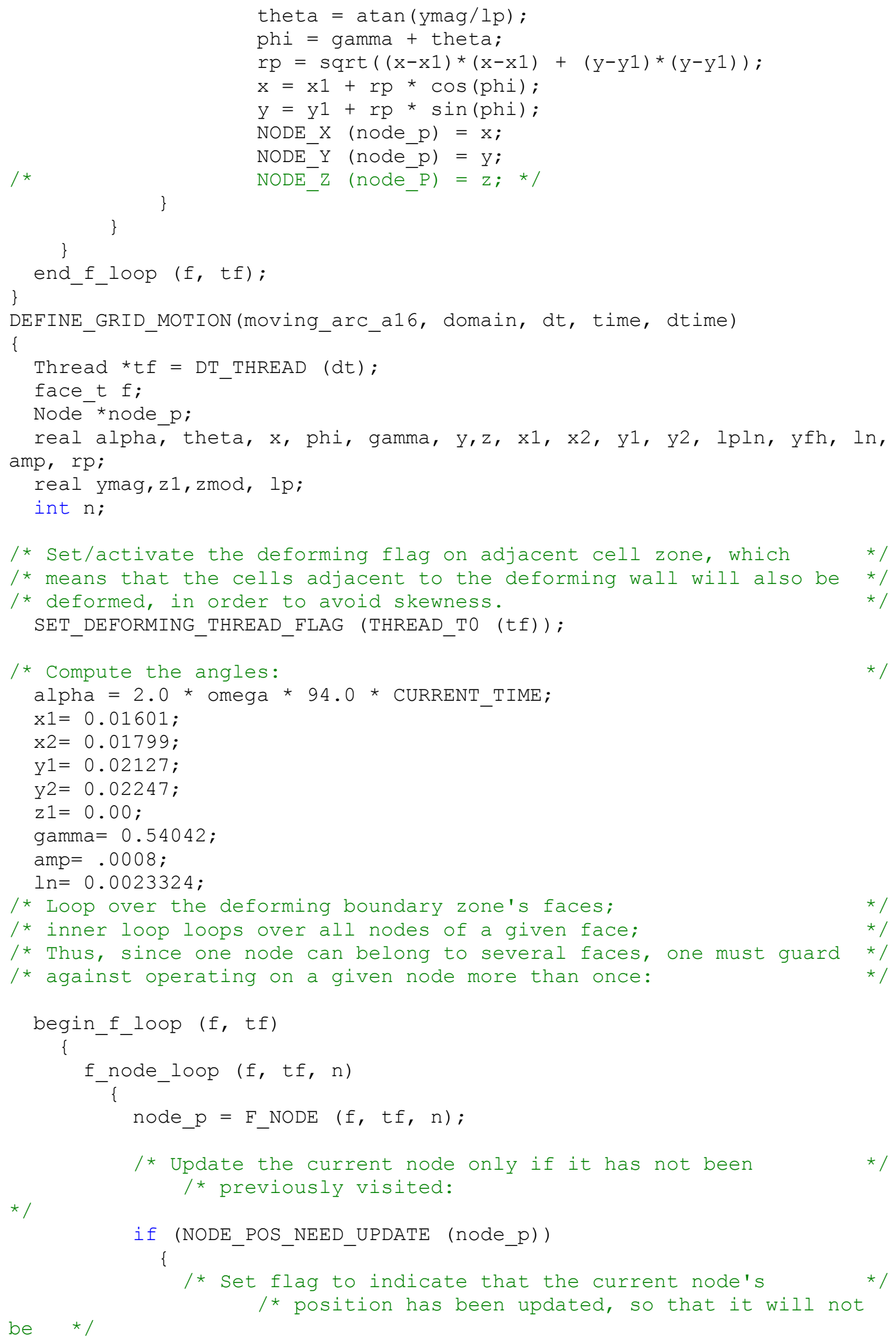




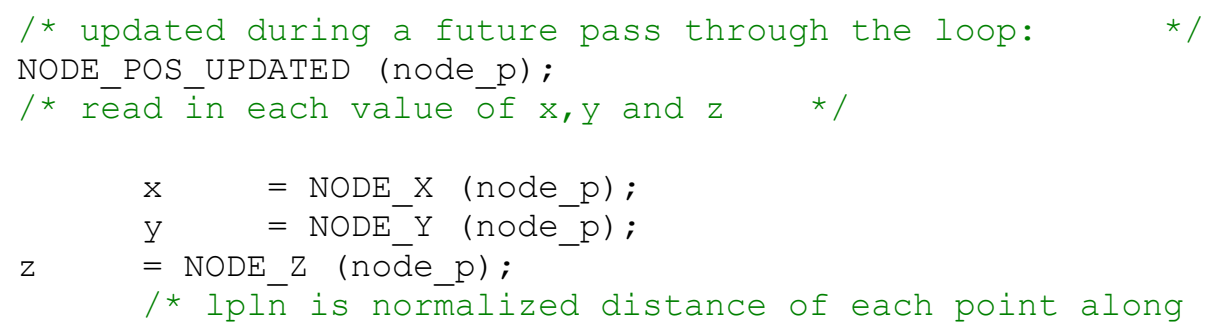




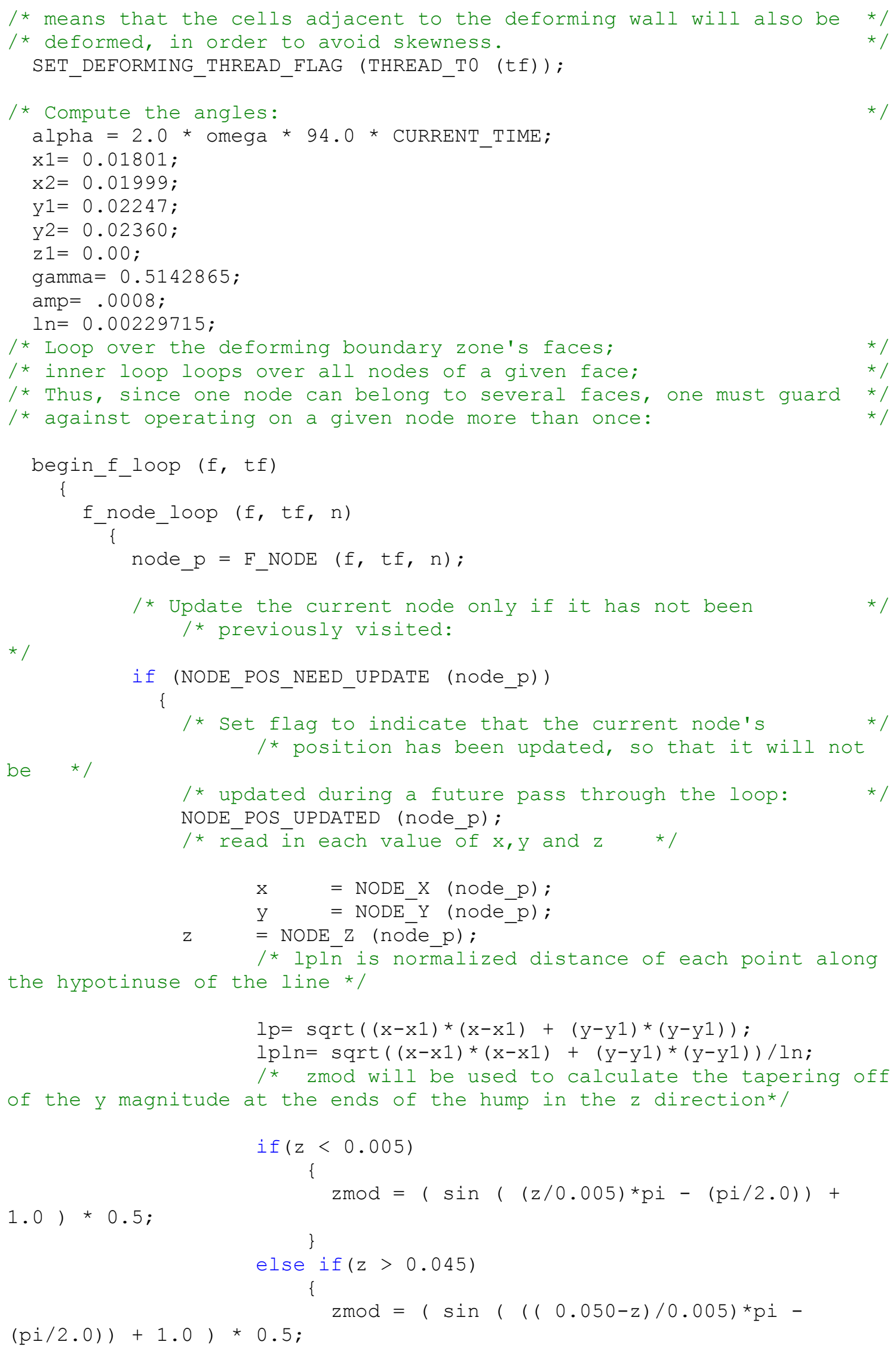




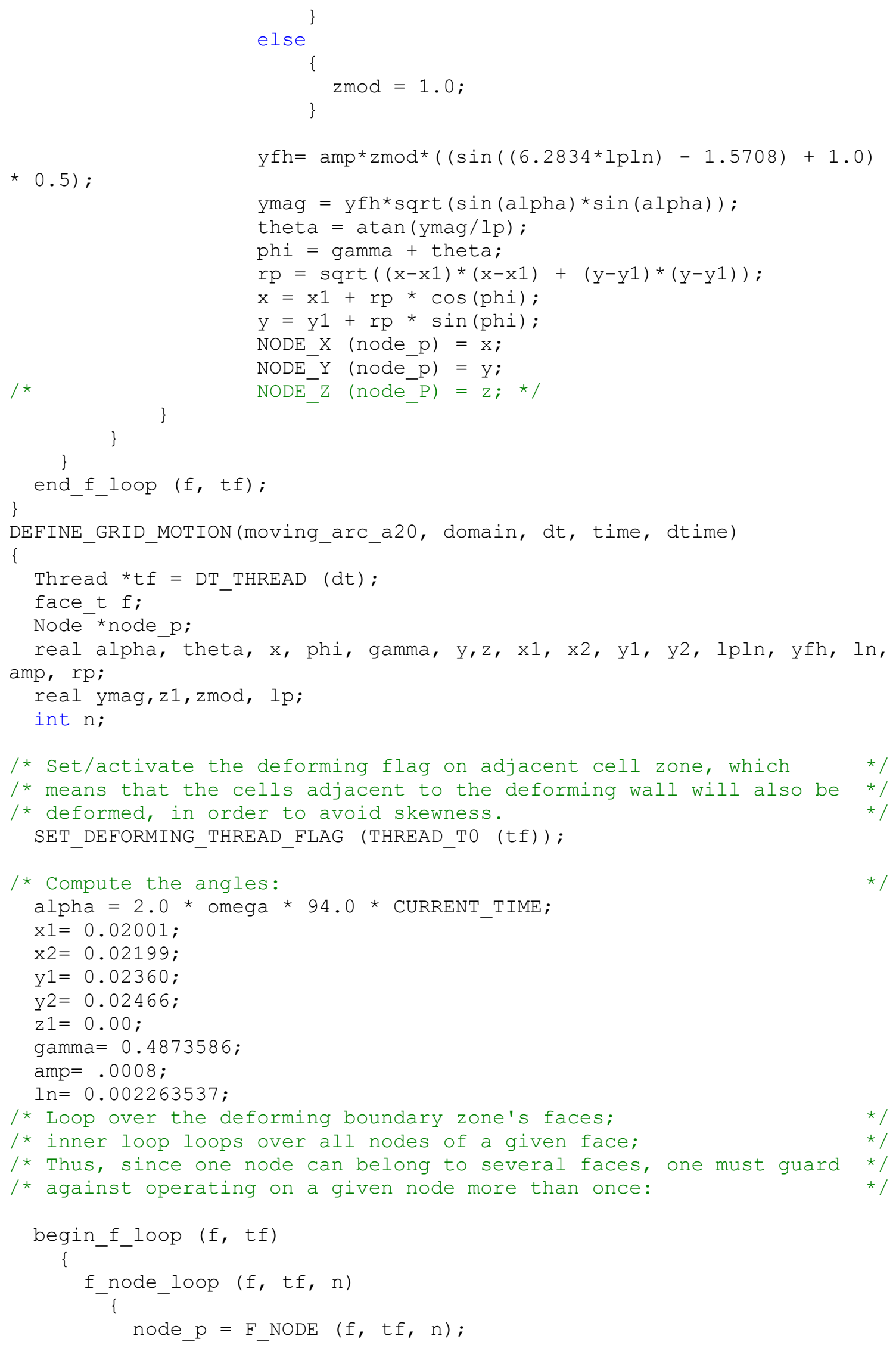




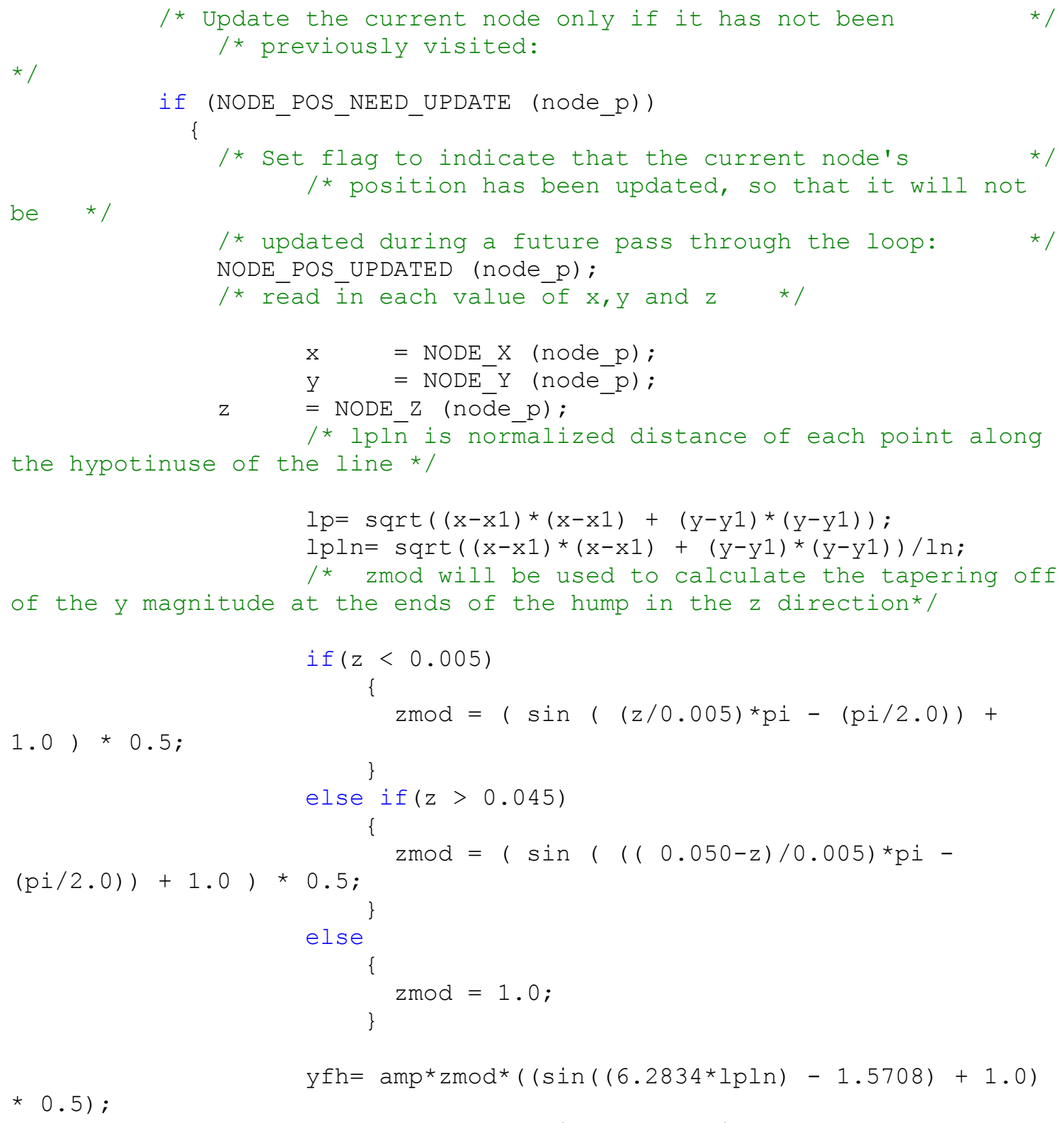




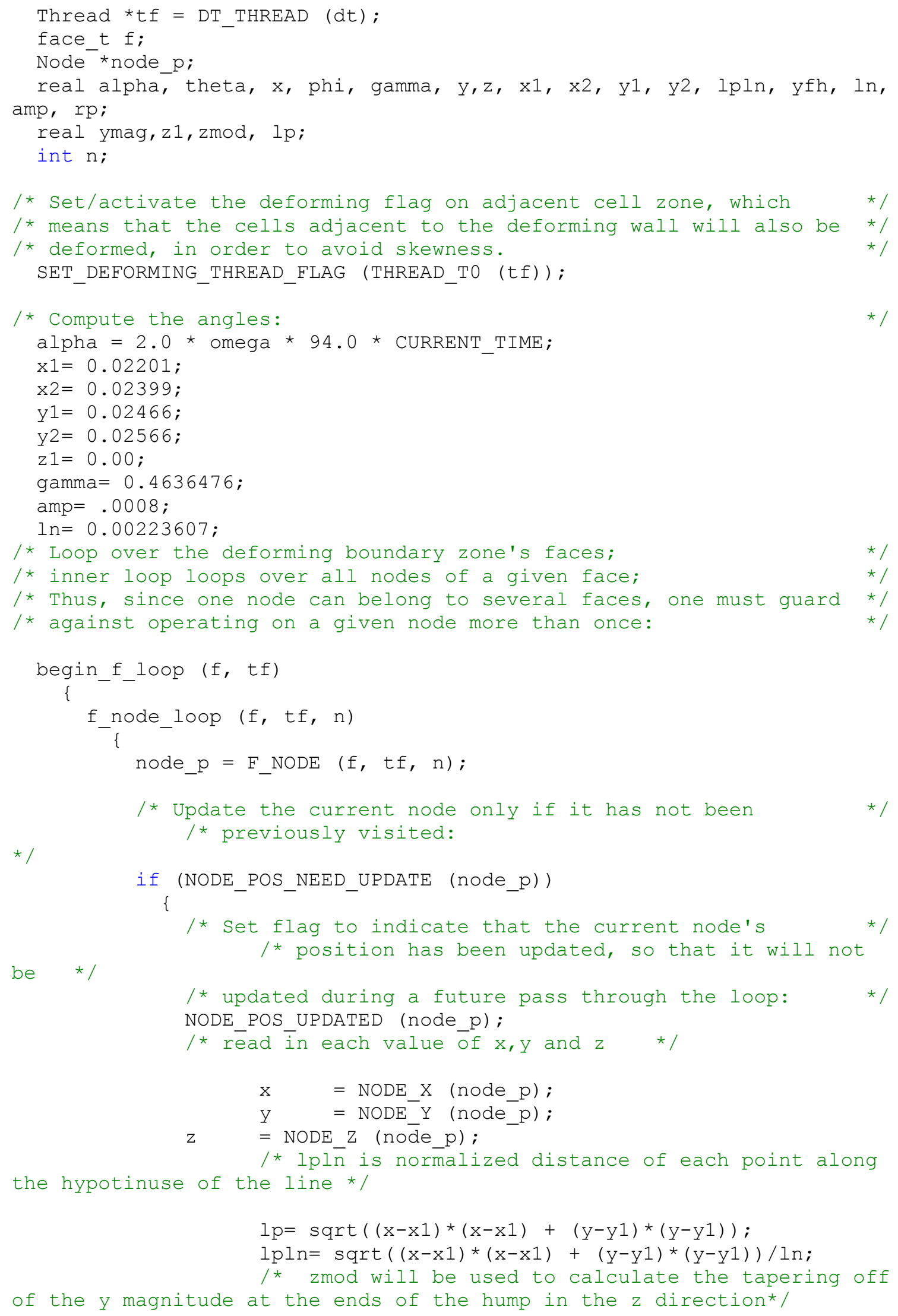




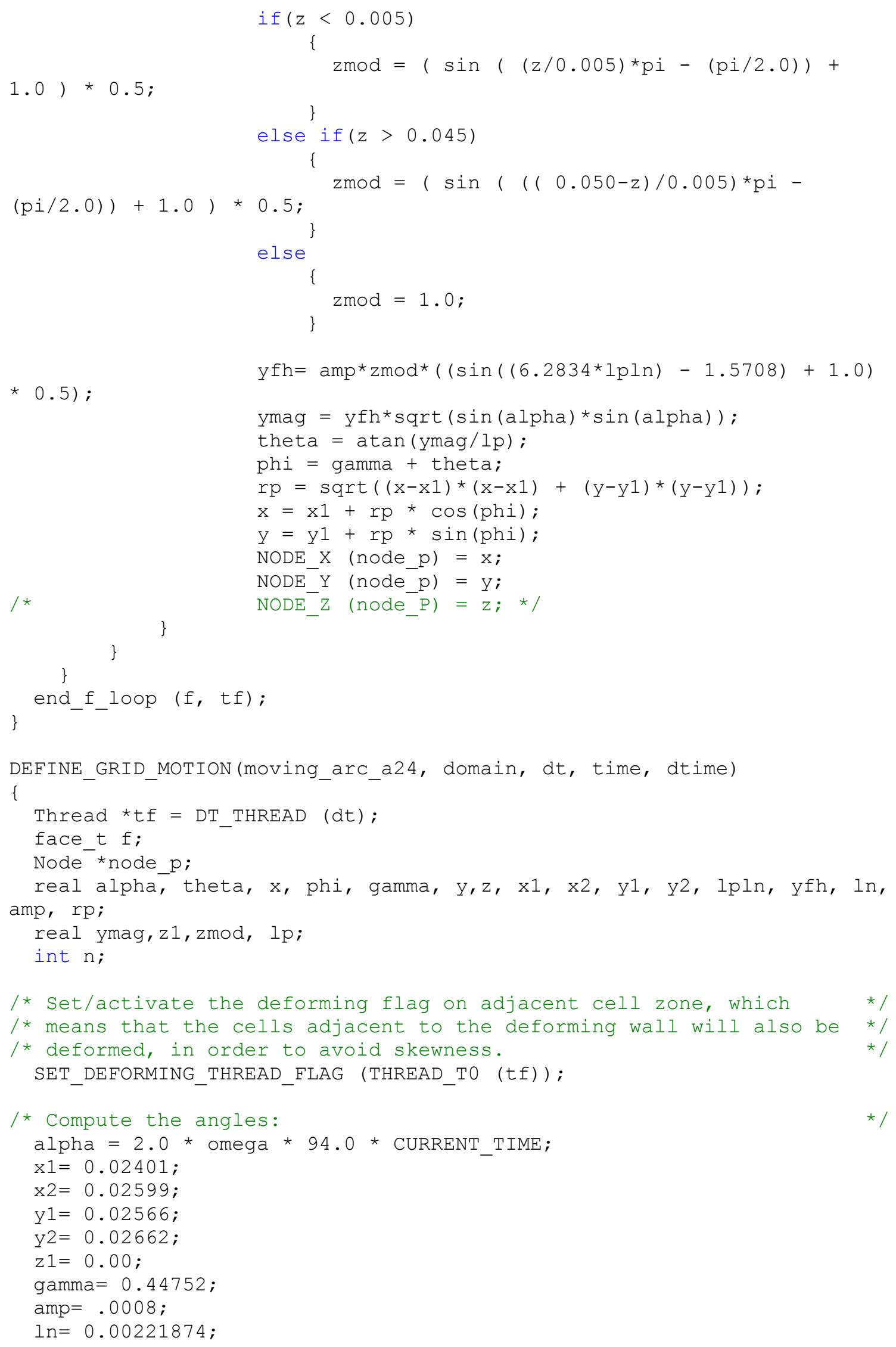




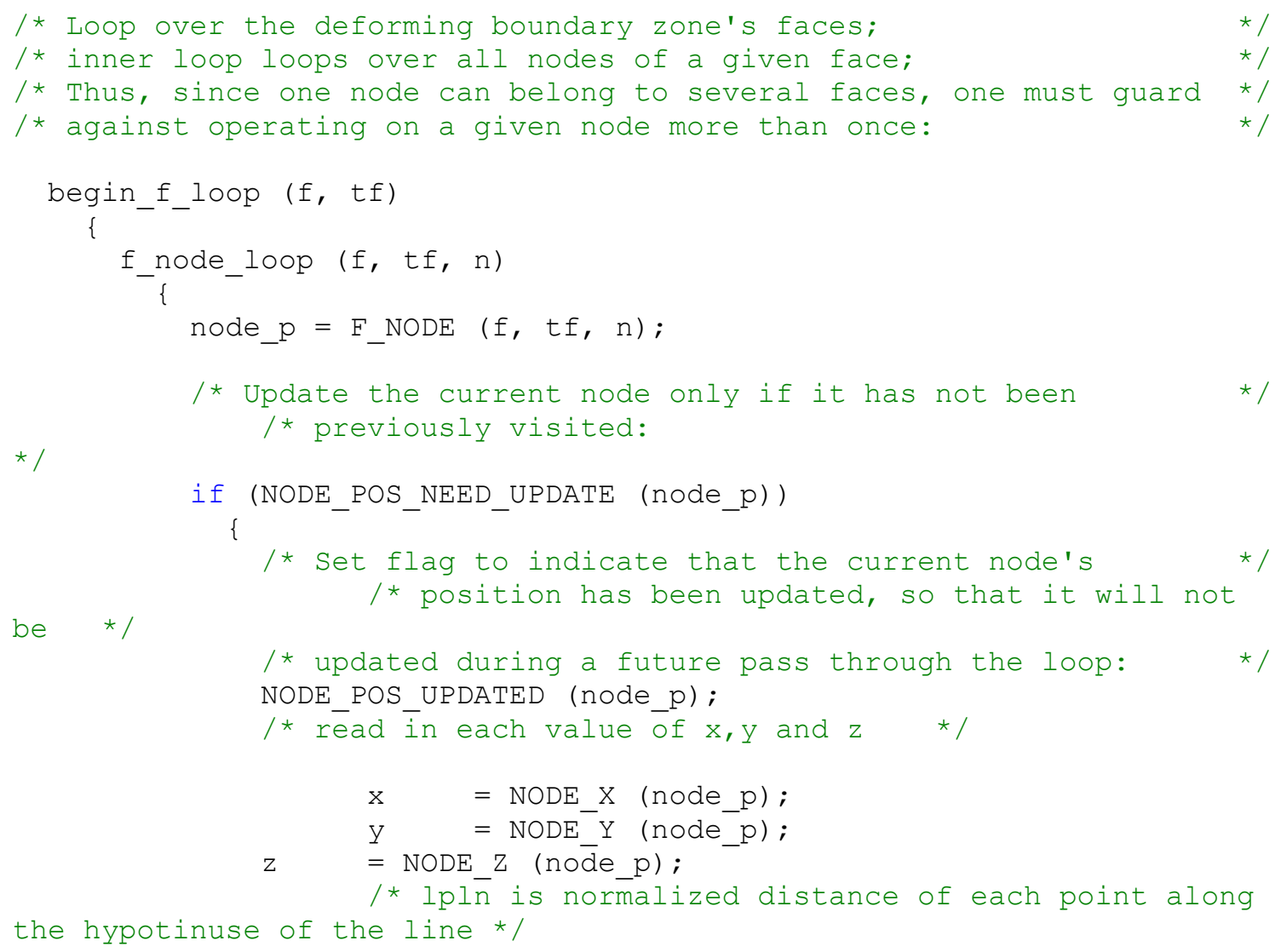




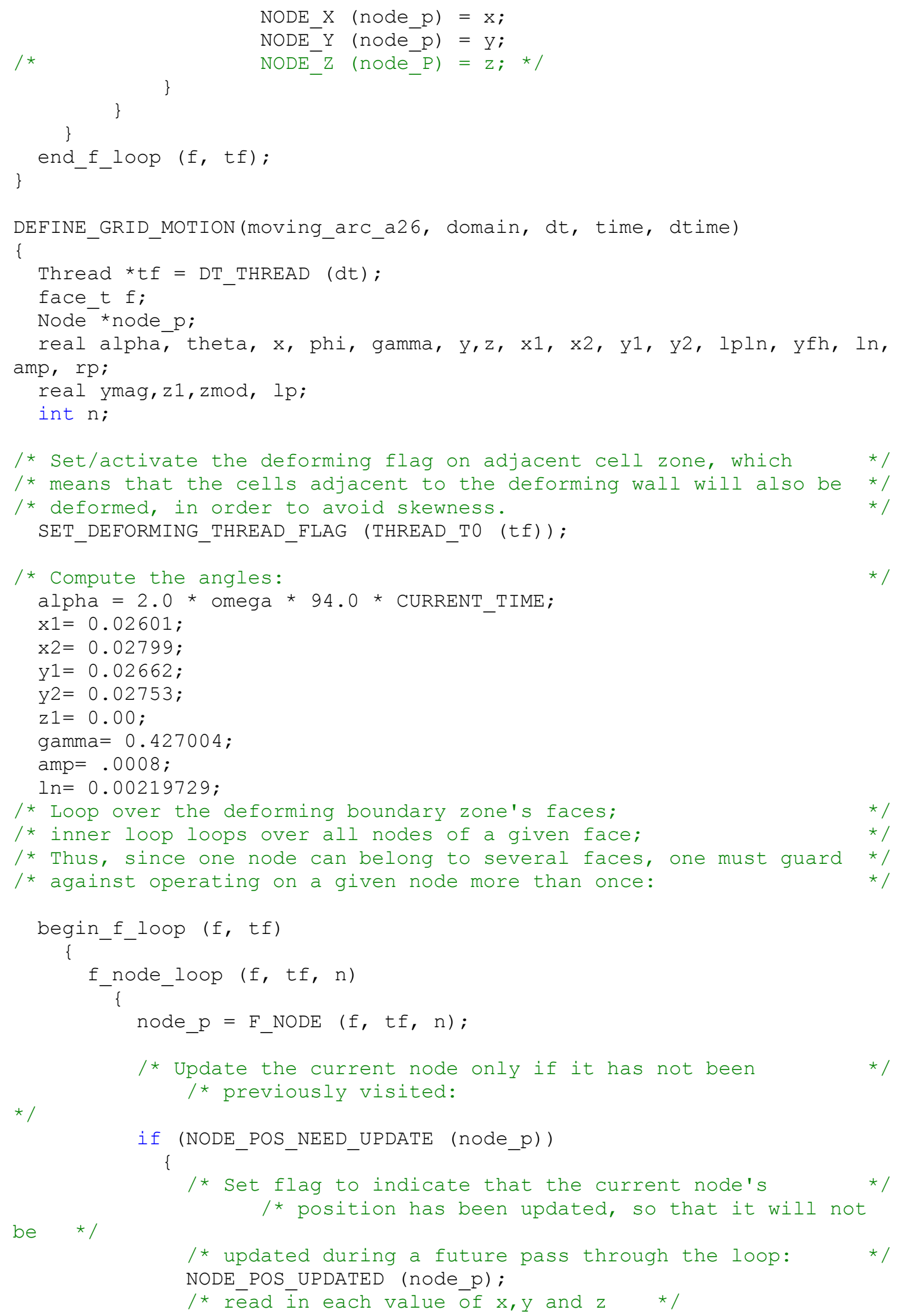




$$
\begin{aligned}
& \mathrm{x} \quad=\text { NODE_X (node_p); } \\
& \mathrm{y} \quad=\text { NODE_Y (node_p); } \\
& \text { z NODE_Z (node_p); } \\
& \text { /* lpln is normalized distance of each point along }
\end{aligned}
$$

the hypotinuse of the line */

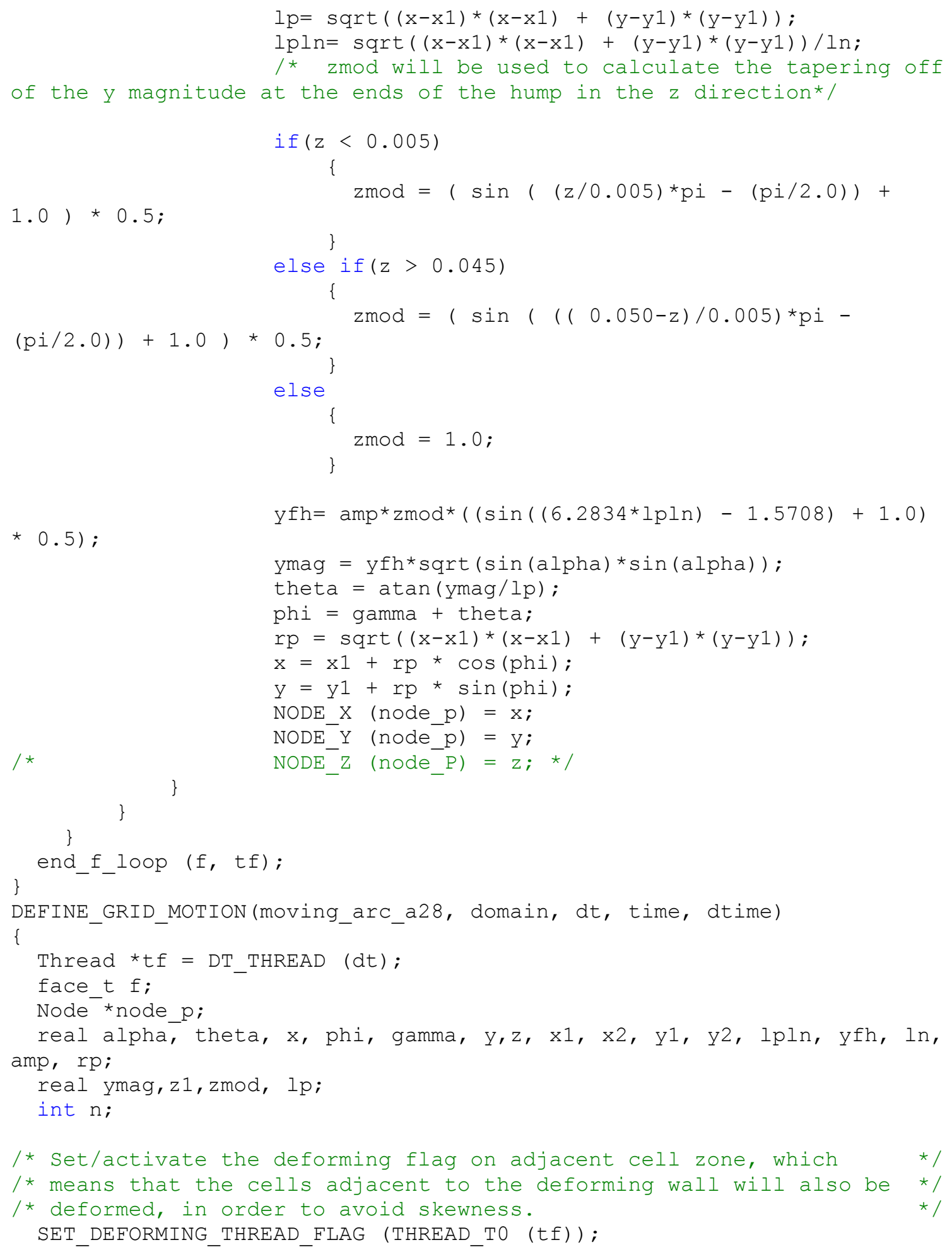




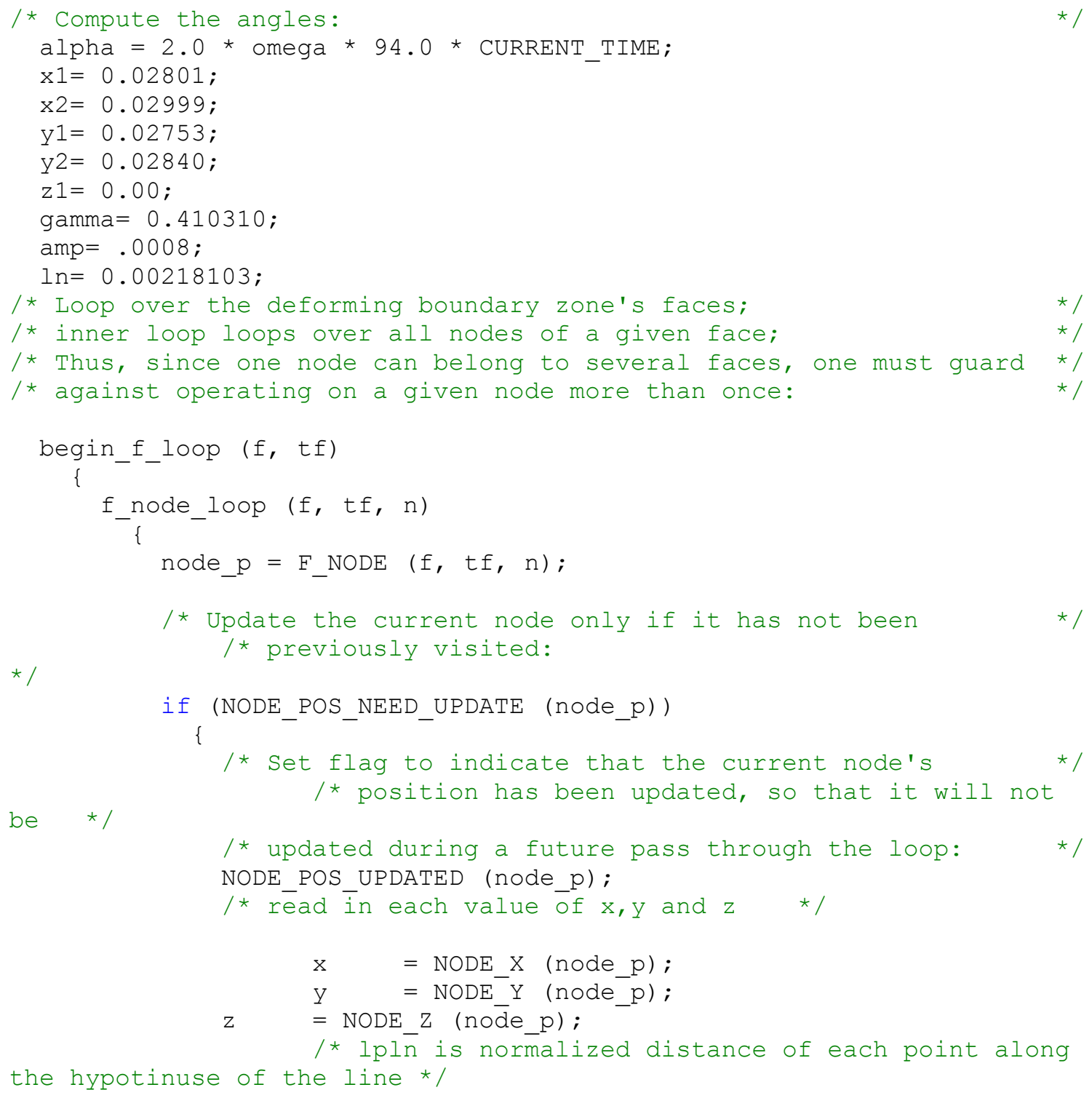


$\star 0.5) ;$

$y f h=a m p * z \bmod *((\sin ((6.2834 * \operatorname{lpln})-1.5708)+1.0)$

ymag $=y f h{ }^{*} \operatorname{sqrt}(\sin (a l p h a) * \sin (a l p h a)) ;$

theta $=\operatorname{atan}(\mathrm{ymag} / \mathrm{lp})$;

phi $=$ gamma + theta;

$r p=\operatorname{sqrt}((x-x 1) *(x-x 1)+(y-y 1) *(y-y 1)) ;$

$\mathrm{x}=\mathrm{x} 1+\mathrm{rp} * \cos (\mathrm{phi}) ;$

$\mathrm{y}=\mathrm{y} 1+\mathrm{rp} * \sin (\mathrm{phi}) ;$

NODE_X (node_p) $=\mathrm{x}$;

NODE_Y (node_p) = $y$;

/*

NODE_Z (node_P) $=z ; *$ /

\}

end_f_loop (f, tf);

DEFINE_GRID_MOTION(moving_arc_a30, domain, dt, time, dtime)

\{

Thread *tf $=$ DT_THREAD (dt);

face_t $f$;

Node ${ }^{*}$ node_p;

real alpha, theta, $x$, phi, gamma, y,z, x1, x2, y1, y2, lpln, yfh, ln, amp, rp;

real ymag, z1, zmod, lp;

int $\mathrm{n}$;

/* Set/activate the deforming flag on adjacent cell zone, which
/* means that the cells adjacent to the deforming wall will also be */
/* deformed, in order to avoid skewness.
SET_DEFORMING_THREAD_FLAG (THREAD_TO (tf));
/* Compute the angles:
alpha $=2.0$ * omega * 94.0 * CURRENT_TIME;

$\mathrm{x} 1=0.03001$

$\mathrm{x} 2=0.03199 ;$

$\mathrm{y} 1=0.02840 ;$

$\mathrm{y} 2=0.02924 ;$

$\mathrm{z} 1=0.00$;

gamma $=0.397628$;

$\mathrm{amp}=.0008$;

$\ln =0.00216924 ;$

/* Loop over the deforming boundary zone's faces; */

/* inner loop loops over all nodes of a given face; */

/* Thus, since one node can belong to several faces, one must guard */

/* against operating on a given node more than once: */

begin_f_loop (f, tf)

\{

f_node_loop (f, $t f, n)$

\{

node_ $\mathrm{p}=\mathrm{F} \_\mathrm{NODE}(\mathrm{f}, \mathrm{tf}, \mathrm{n})$;

/* Update the current node only if it has not been */

*

/* previously visited: 


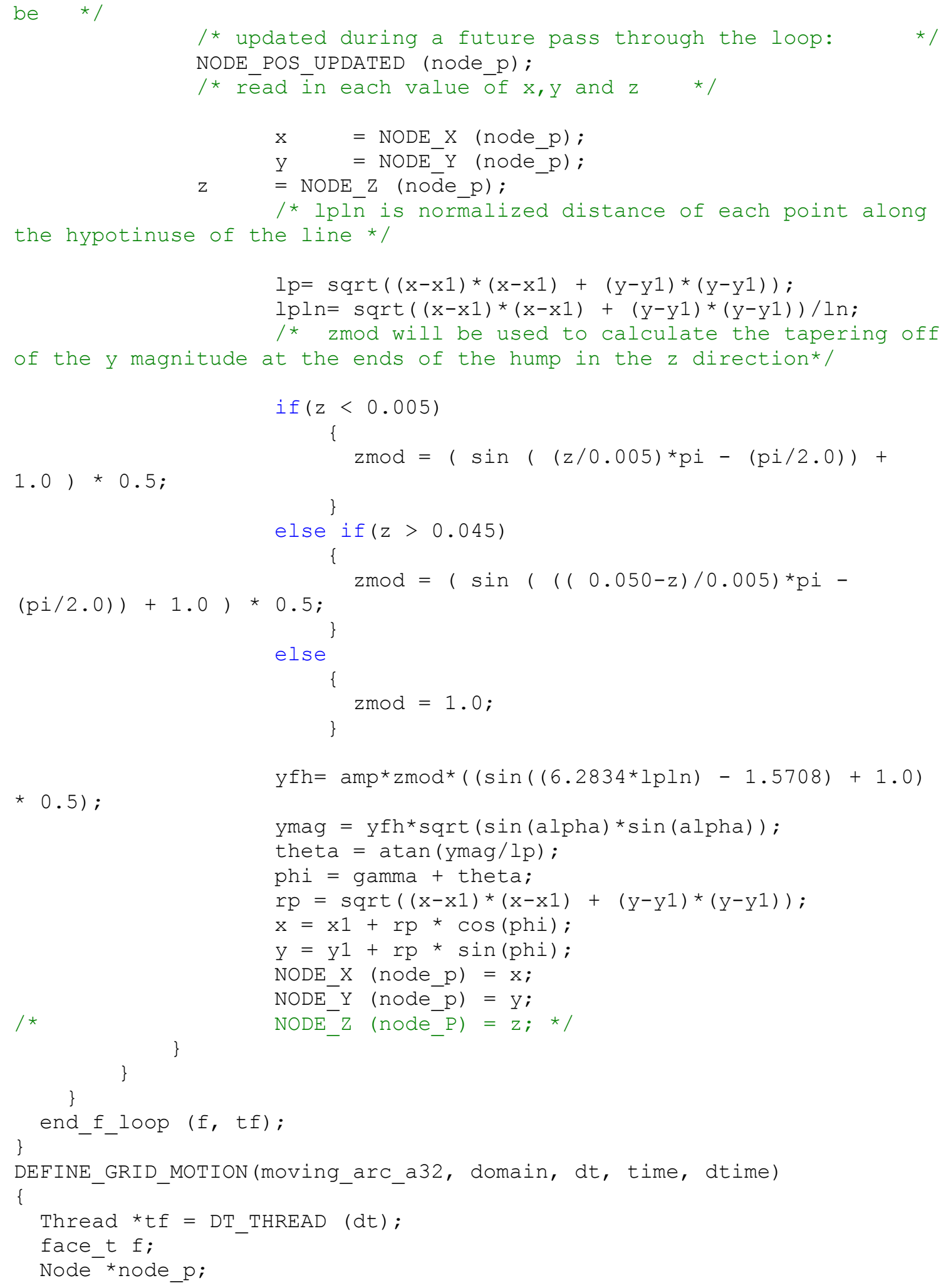




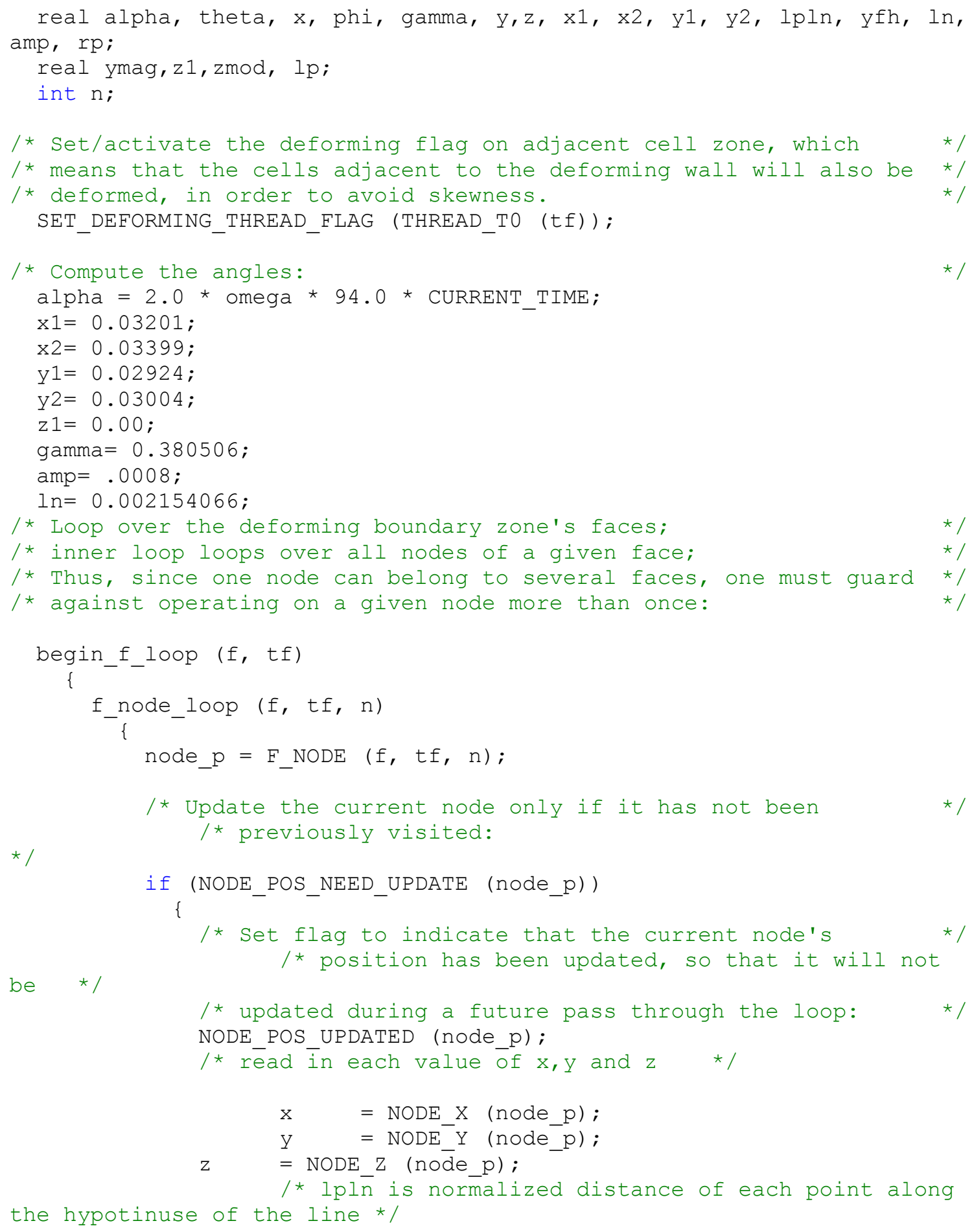




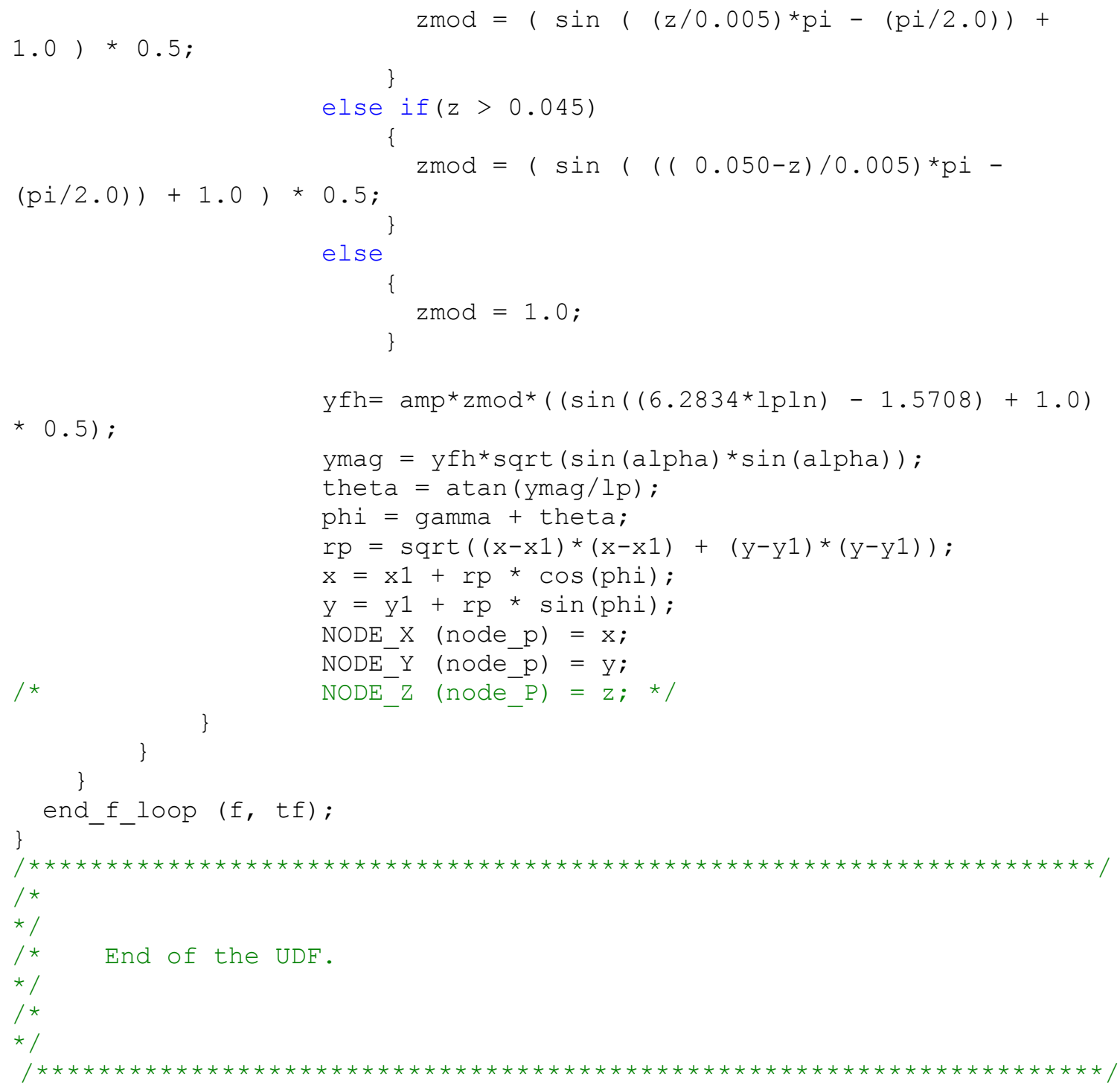

